\title{
The impact of the mitochondrial bottleneck on mtDNA disease risk and embryogenesis
}

Citation for published version (APA):

Otten, A. B. C. (2016). The impact of the mitochondrial bottleneck on mtDNA disease risk and embryogenesis. [Doctoral Thesis, Maastricht University]. Ipskamp. https://doi.org/10.26481/dis.20161115ao

Document status and date:

Published: 01/01/2016

DOI:

10.26481/dis.20161115ao

Document Version:

Publisher's PDF, also known as Version of record

\section{Please check the document version of this publication:}

- A submitted manuscript is the version of the article upon submission and before peer-review. There can be important differences between the submitted version and the official published version of record.

People interested in the research are advised to contact the author for the final version of the publication, or visit the DOI to the publisher's website.

- The final author version and the galley proof are versions of the publication after peer review.

- The final published version features the final layout of the paper including the volume, issue and page numbers.

Link to publication

\footnotetext{
General rights rights.

- You may freely distribute the URL identifying the publication in the public portal. please follow below link for the End User Agreement:

www.umlib.nl/taverne-license

Take down policy

If you believe that this document breaches copyright please contact us at:

repository@maastrichtuniversity.nl

providing details and we will investigate your claim.
}

Copyright and moral rights for the publications made accessible in the public portal are retained by the authors and/or other copyright owners and it is a condition of accessing publications that users recognise and abide by the legal requirements associated with these

- Users may download and print one copy of any publication from the public portal for the purpose of private study or research.

- You may not further distribute the material or use it for any profit-making activity or commercial gain

If the publication is distributed under the terms of Article $25 \mathrm{fa}$ of the Dutch Copyright Act, indicated by the "Taverne" license above, 


\section{The impact of the mitochondrial bottleneck \\ on mtDNA disease risk and embryogenesis}

Auke Bernardus Catharina Otten 


\section{Maastricht UMC+}
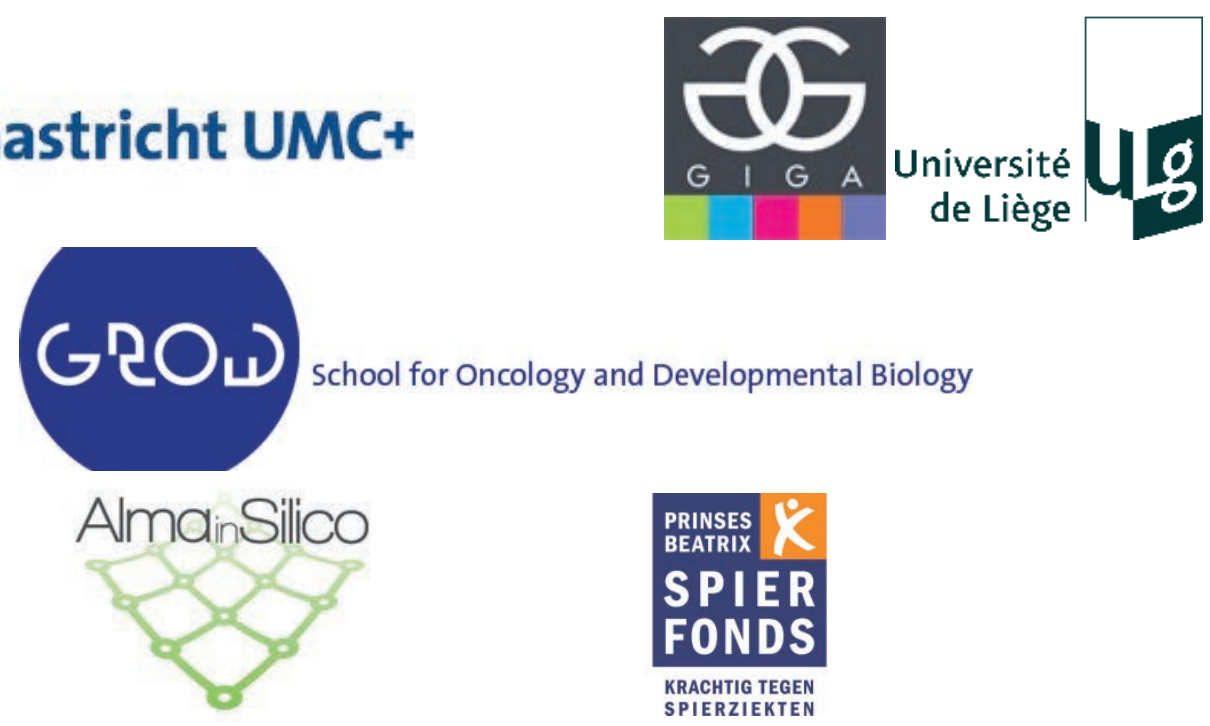

The study presented in this thesis was performed within the GROW School for Oncology and Developmental Biology. Research was carried out at the department of Genetics and Cell Biology (Faculty of Health, Medicine and Life Sciences of Maastricht University Medical Centre) and the GIGA Research Centre (Université de Liège, Belgium).

The studies described in this thesis were supported by the the Princes Beatrix Foundation and the euregional Alma-in-Silico platform, which are gratefully acknowledged by the author.

(C) Auke B. C. Otten, Maastricht, 2016

ISBN 978-94-028-0365-5

Cover design: $\quad$ Suren Nersisyan \& Frank van Opdorp

Lay-out:

Marten Hoeksema \& Auke Otten

Printed by:

Ipskamp printing || www.proefschriften.net

Published by: Ipskamp BV 


\title{
The impact of the mitochondrial bottleneck \\ on mtDNA disease risk and embryogenesis
}

\section{Studies in humans and zebrafish}

\author{
Proefschrift \\ ter verkrijging van de graad van doctor \\ aan de Universiteit Maastricht \\ op gezag van de Rector Magnificus \\ Prof. Dr. Rianne M. Letschert \\ volgens het besluit van het College van Decanen \\ in het openbaar te verdedigen \\ op dinsdag 15 november 2016 om 14:00 uur
}

door

Auke Bernardus Catharina Otten

Geboren te Sittard op 3 juli 1986 
Promotor

Prof. Dr. H.J.M. Smeets

\section{Copromotor}

Dr. M. Muller

(Université de Liège, BE)

Beoordelingscommissie

Prof. Dr. J.P.M. Geraedts

(voorzitter)

Prof. Dr. Ir. E.A.L. Biessen

Prof. Dr. P.F. Chinnery

(University of Cambridge, UK)

Prof. Dr. F. Dequiedt

(Université de Liège, BE)

Prof. Dr. W.H. Lamers 
To reach the end Of your known world And to go that bit further To kick your ball in the wilderness And to follow

From: Mackenzie Thorpe 



\section{Contents}

Chapter 1 General Introduction \& Thesis Outline

Chapter 2 Evolutionary origin of and mechanisms in mitochondria

Chapter 3 Differences in strength and timing of the mtDNA bottleneck between zebrafish germline and non-germline cells

Chapter 4 Zebrafish oocytes with a low mtDNA copy number are at risk for de novo mutations reaching functional significance

Chapter 5 Mitochondrial DNA inheritance is determined by genetic drift and negative and positive selection

Chapter 6 Tfam knockdown causes a developmental defect of heart and brain due to energy deficit and mitochondrial stress response 

Chapter 1

General Introduction \&

Thesis Outline 


\section{Mitochondrial disorders and inheritance}

Defective mitochondrial function is associated with a wide range of clinical manifestations, including encephalopathies, myopathies and cardiomyopathies. This is not surprising given that mitochondria are, amongst others, responsible for the production of the majority of cellular energy (such as adenosine triphosphate (ATP)). As the brain, skeletal muscle and heart are organs with high energy demands, these organs are specifically sensitive to dysfunctional mitochondria. Mitochondrial diseases are often multi-systemic, as in the MELAS (Mitochondrial Encephalomyopathy, Lactic Acidosis, and Stroke-like episodes) syndrome, which is characterized by several neurologic manifestations, but the same genetic defect can also lead to cardiac conduction defects, diabetes mellitus, deafness, short stature, myopathy, renal failure and gastrointestinal disturbances ${ }^{1}$. In some cases, mitochondrial disease can affect only a single tissue, such as the optic nerve in Leber's hereditary optic neuropathy (LHON). In fact, any organ can be affected at any age, depicting the high variability of manifestation of mitochondrial disorders. The underlying genetic defect generally causes a deficiency of the oxidative phosphorylation (OXPHOS, Figure 1.1), the terminal component of mitochondrial energy metabolism, and can be present in both the mitochondrial DNA (mtDNA), which segregates maternally, and in the nuclear DNA (nDNA), which segregates in a Mendelian fashion. The total prevalence of adult mitochondrial diseases, caused by pathogenic mutations in either the nDNA or mtDNA, has been estimated 1 in 4,300 and the minimal prevalence of mtDNA disease was 1 in $5,000^{2}$. Of the mtDNA mutations, $\sim 25 \%$ is caused by de novo mutations ${ }^{3}$. The extreme phenotypic and genetic variability (Figure 1.1) associated with mitochondrial disorders hampers clinical practice and diagnosis, as well as the development of treatment options, which generally do not exist for these patients.

\section{Mitochondrial DNA genetics and inheritance}

The mtDNA contains 37 genes, of which 13 are protein-coding. It is a multicopy genome and cells maintain a high mtDNA copy number. As a result, a mixture of wild-type and variant or mutant mtDNA generally co-exists in mtDNA patients, a state referred to as heteroplasmy. mtDNA disease manifests when the heteroplasmy level exceeds a certain threshold. This threshold is variable and is influenced by, amongst others, mutation type, tissue type and maternal age (Figure 1.2). Due to the maternal inheritance, only offspring of female mtDNA carriers is at risk of developing inherited mtDNA disease. During segregation, the heteroplasmy levels between mother and children can change considerably ${ }^{4}$. This is mainly due to a genetic bottleneck that occurs during mtDNA transmission. This is a restriction in the number of mtDNA molecules transmitted to subsequent generations ${ }^{5}$, followed by a strong amplification of these limited founder molecules. In healthy cases, this system prevents the accumulation of acquired mtDNA mutations and a return to (healthy ${ }^{6}$ ) homoplasmy is established within a single generation ${ }^{4}$. However, when a female carries a disease-causing mtDNA mutation this bottleneck can have an adverse effect, resulting in unpredictable transmission of highly variable heteroplasmy levels (Figure 1.2). 




Figure 1.1 | The genetic and phenotypic heterogeneity of mitochondrial disorders. The mitochondrial oxidative phosphorylation (OXPHOS) is under dual genetic control, as the proteins for the OXPHOS machinery lay encoded in both the mitochondrial DNA (mtDNA) and the nuclear DNA. Apart from complex subunits of the OXPHOS, the nDNA also encodes for genes necessary to assemble the subunits, genes controlling mtDNA maintenance (e.g. replication), genes controlling mtDNA translations and genes for indirect OXPHOS proteins. Currently, in total 1,158 nuclear and mtDNA genes with strong support of mitochondrial localization have been reported ${ }^{7}$. Mitochondrial disorders caused by a nuclear gene defect inherit in a Mendelian fashion, while those caused by a mitochondrial gene have a maternal inheritance pattern. Besides this genetic heterogeneity, mitochondrial disorders can have a wide variety of clinical symptoms, causing phenotypic variability, as almost any organ can be affected.

The processes underlying mtDNA inheritance are not precisely known, and different hypotheses have been postulated concerning the mechanism of mtDNA segregation, which could involve random genetic drift $^{8}$ and/or selection ${ }^{9}$. Dependent on the mutation and whether it is familial or de novo, either prenatal diagnosis (PND) or preimplantation genetic diagnosis (PGD) can be offered to female carriers of mtDNA mutations to prevent disease transmission. Unravelling the mechanisms underlying the inheritance of mtDNA mutations are important for understanding and predicting occurrence, manifestation and transmission of mtDNA mutations and can lead to improved options for preventing recurrence and to more accurate counselling of patients and carriers. This may also open up new opportunities to intervene. 


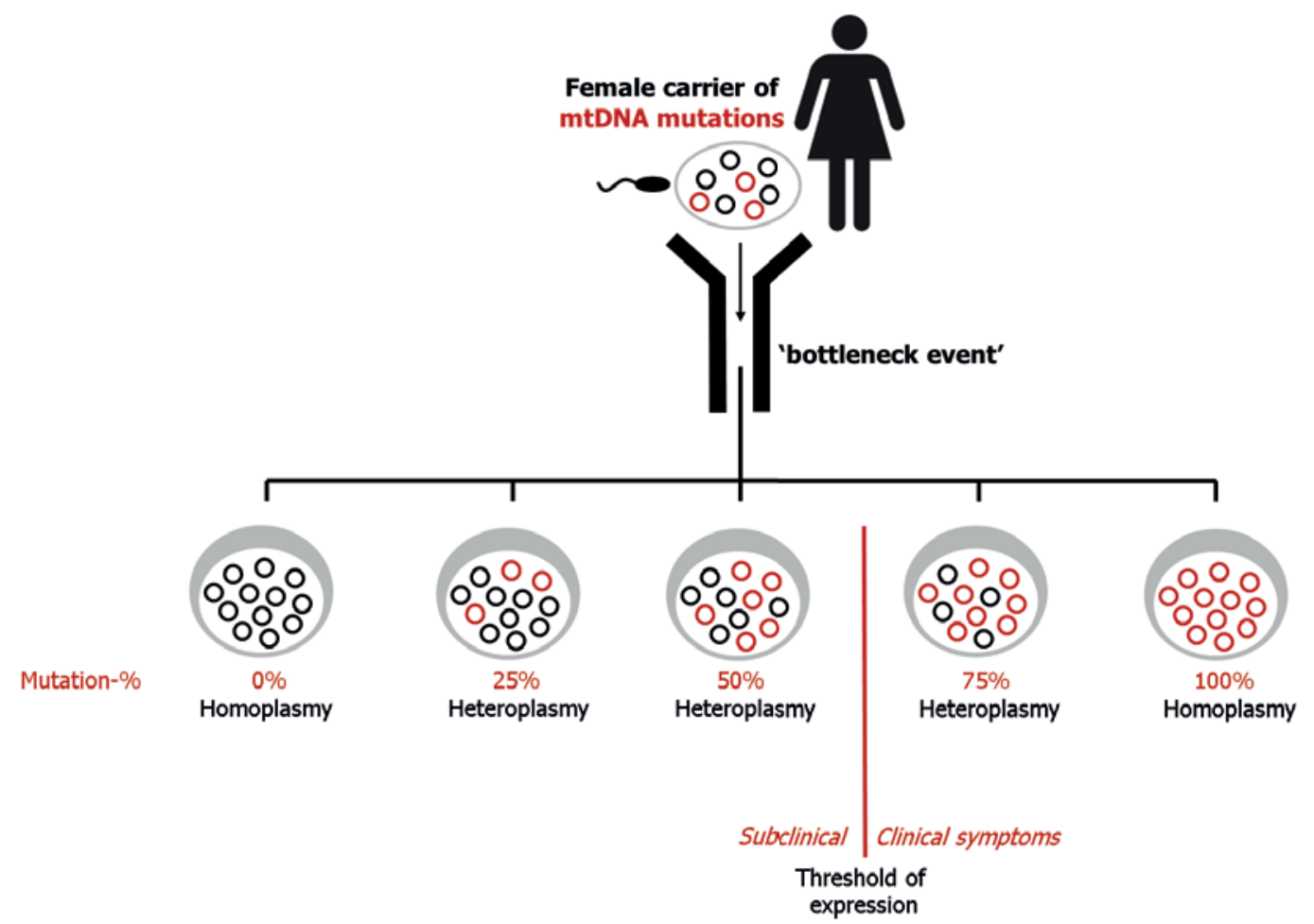

Figure 1.2 | Inheritance of heteroplasmic mtDNA mutations. As a cell contains multiple copies of mtDNA, a mixture of wild-type and variant or mutant mtDNA can co-exist, a state referred to as heteroplasmy. If a female carries an mtDNA mutation, her offspring can inherit heteroplasmy values ranging from $0 \%$ to $100 \%$, prohibiting accurate predictions of the mtDNA mutations load in the offspring. mtDNA diseases only manifest if the heteroplasmy level exceeds a disease threshold, which varies among mtDNA mutations.

\section{Zebrafish models to study mtDNA inheritance}

Characterizing the mechanism of mtDNA inheritance in humans has considerable limitations. It relies mainly on studying the mtDNA load in pedigrees and in a limited number of available oocytes or embryos from carriers ${ }^{10}$. Furthermore, population based epidemiological genetic studies have been performed to estimate the prevalence and recurrence risk of mtDNA disease ${ }^{2 ; 11 ; 12}$, but these human studies do not allow interventions or detailed mechanistic evaluations in significant numbers of samples. Ample opportunities to study mtDNA segregation are offered through study studies in animals, ranging from cows ${ }^{4}$ and primates ${ }^{13}$ to 'traditional' mice and rats $^{5}$. Especially mouse models have been extensively used to study mtDNA inheritance, and heteroplasmic mouse models have been generated by introducing polymorphic mtDNA from the NZB mouse into laboratory inbred strains. Subsequently, the proportion of NZB mtDNA can be measured in different cell types over multiple generations to study mtDNA inheritance ${ }^{5 ; 6 ; 14-16}$. Although human and animal studies have provided essential knowledge on mtDNA segregation, the picture is still far from complete and additional models that allow easy and fast evaluation of inheritance parameters in a large number of samples are still required. 
The zebrafish (Danio rerio) is a small tropical freshwater fish with a natural distribution from the Indian peninsula to the Himalayas ${ }^{17}$ It is a vertebrate offering ample opportunities for research, especially as a model for human development and disease. Zebrafish are generally $3-4 \mathrm{~cm}$ when fully grown ${ }^{18}$ and require little care and space, resulting in low maintenance costs ${ }^{19}$. Being a vertebrate model, nearly all human organ systems are present in the zebrafish, especially organs frequently affected in mitochondrial disorders, like the brain, heart and muscle, are well developed ${ }^{20}$. Due to its rapid development, all primary organs are already present after 3-5 days post fertilization (dpf). As female zebrafish deposit their eggs in the water, males fertilize the eggs externally and development takes place ex-utero. This makes the embryos easily accessible for observation and zebrafish embryogenesis is well described ${ }^{18}$. Another striking feature of zebrafish development is the transparency of embryos and larvae, which greatly facilitates imaging ${ }^{19 ; 20}$. The zebrafish model offers opportunities to apply efficient invertebrate-style genetics to vertebrate-related questions ${ }^{19}$, allowing many forward- and reverse-genetics approaches. Consequently, the zebrafish is a model system with increasing popularity to study basic developmental, genetic, fertility, cell biological and biochemical processes, as well as for modelling human diseases ${ }^{19}$.

The nuclear genome of the zebrafish has been sequenced completely and has revealed that $>70 \%$ of the human genes have at least one obvious zebrafish orthologue ${ }^{21}$. Regarding the mtDNA, the zebrafish mitochondrial genome contains the same 37 genes as the human counterpart with $\sim 70 \%$ sequence identity, making it is also a good model to study mtDNA mutations and mtDNA inheritance. The mtDNA bottleneck is highly conserved within the animal kingdom ${ }^{22}$. The zebrafish offers the possibility to isolate large amounts of the primordial germ cells (PGCs) oocytes and somatic cells, enabling a detailed mechanistic analysis of the mtDNA bottleneck.

\section{Aims and thesis outline}

The mtDNA bottleneck is the predominant mechanism occurring during the inheritance of the mtDNA in order to prevent acquired mutations to accumulate, thereby keeping mtDNA mutation frequency at an evolutionary acceptable level and ensuring a preferentially healthy mtDNA genome being transmitted from mother to child. The processes underlying the mtDNA bottleneck are still largely unknown. The bottleneck can have an adverse effect for carriers of heteroplasmic disease-causing mtDNA mutations, leading to unpredictable, possibly high heteroplasmy levels in their oocytes, and could possibly explain the high de novo mtDNA mutation frequency. The overall aim of this thesis is to characterize the processes that underlie mtDNA segregation through the mtDNA bottleneck and to explain the de novo mtDNA disease risk.

Specific aims are:

- to define the size of the mtDNA bottleneck during transmission;

- to investigate the risk of acquiring de novo mtDNA mutations during oogenesis;

- to identify the biological processes that underlie the mtDNA bottleneck and the (mutation-specific) shifts in mtDNA heteroplasmy levels between mothers and children;

- to study the effect of a low mtDNA copy number on embryogenesis. 
In chapter 2 we evaluated the role and importance of the mtDNA and the mtDNA bottleneck by reviewing the evolution of the mtDNA genome in animals, plants and fungi. Mitochondria are derived from the endosymbiosis of an alpha-bacterium and a eubacterium billions of years ago and mitochondria contain their own genetic material as a result. In almost all species, the mtDNA has uniparental (mostly maternal) asexual inheritance patterns, which puts the mtDNA genome at risk of fatal mutation accumulation due to the mitochondrial ROS production, a process referred to as Muller's ratchet. To avoid genomic decay and eventually extinction, solutions to prevent detrimental mutation accumulation have evolved in parallel. The main mechanism, occurring in all species, is endosymbiotic gene transfer (EGT), which has drastically reduced the mtDNA genome size in animals, plants and fungi. Still, all mtDNA genomes display mutation rates that are higher than the nuclear genome. After the phylogenetic divergence of eukaryotes in animals, fungi and plants, differences in mtDNA evolution have occurred to cope with the mutational burden. While in plants the mtDNA mutation rate is kept low by effective ROS defence and efficient recombination-mediated mtDNA repair, these mechanisms are not or less-well developed in animals. As a result, the mtDNA mutation rate in animals is higher compared to plants. This might be evolutionarily favourable, as it allows mobile animals to adapt more rapidly to changes in environmental conditions in terms of energy production. The detrimental mutagenesis events in animals are balanced by a high mtDNA copy number in combination with a purifying genetic bottleneck during transmission. This purifying bottleneck serves as an important evolutionary mechanism to prevent mutation accumulation in the animal mtDNA, but the factors defining the bottleneck or the underlying molecular mechanisms are currently unknown.

In chapters 3-5 we provide insight in the size, timing and mechanisms of the bottleneck. In chapter 3 we characterized the size and timing of mtDNA bottleneck in zebrafish, as well as differences between germline and non-germline cells. We used a transgenic line allowing efficient isolation of (non-)germline cells from zebrafish embryos at various stages of development and found a strong reduction in the mtDNA copy number during early embryogenesis. We hypothesized that a low copy at the bottom of the bottleneck creates an increased risk for de novo mutations reaching functional significant heteroplasmy values. Therefore, we characterized the de novo mutation load in relation to the bottleneck size variation in chapter 4 . We sequenced the mtDNA of somatic tissues and oocytes from female zebrafish using state-of-the art deep sequencing techniques. We compared the maternal somatic mtDNA sequences with those of her oocytes to identify and quantify the de novo mtDNA mutations. In chapter $\mathbf{5}$ we studied human oocytes and embryos of female mtDNA mutation carriers undergoing preimplantation genetic diagnosis (PGD) to characterize the biological processes responsible for the rapid shifts in mtDNA heteroplasmy observed during mtDNA transmission. Apart from fundamental understanding of this process, this information aids in a better prediction of the recurrence risk for female carriers of mtDNA mutations. As sufficient mitochondria and mtDNA molecules are essential for normal embryonic development, healthy fertilization and compensating germline and somatic de novo mutations, we studied, finally, in chapter $\mathbf{6}$, the effect of defective mtDNA replication during zebrafish development by knocking down one of the key genes initiating mtDNA replication (Tfam). We performed global expression analysis to identify the underlying pathological mechanisms.

In the general discussion (chapter 7), we discuss how the mitochondrial 
evolutionary mechanisms, identified in chapter 2, including the mtDNA bottleneck; can lead to adverse health effects in individuals. As the mtDNA copy number is important for successful fertilization and embryogenesis (chapter 6), the mitochondria are also crucial in reproductive biology and medicine. Genetic tools in zebrafish are rapidly developing and disease models caused by mtDNA mutations could be developed in order to assess the impact of these mutations, including their genotype-phenotype relation. Furthermore, the processes that underlie the inheritance and expression of mtDNA mutations could then be studied in more detail. Such information can be used for better understanding and counselling of mtDNA disorders, as well as for the development and testing of novel therapeutic and preventive options for these devastating diseases.

\section{References}

1. Santa, K.M. (2010). Treatment options for mitochondrial myopathy, encephalopathy, lactic acidosis, and stroke-like episodes (MELAS) syndrome. Pharmacotherapy 30, 1179-1196.

2. Gorman, G.S., Schaefer, A.M., Ng, Y., Gomez, N., Blakely, E.L., Alston, C.L., Feeney, C., Horvath, R., Yu-Wai-Man, P., Chinnery, P.F., et al. (2015). Prevalence of nuclear and mitochondrial DNA mutations related to adult mitochondrial disease. Annals of neurology 77, 753-759.

3. Sallevelt, S.C., de Die-Smulders, C.E., Hendrickx, A.T., Hellebrekers, D.M., de Coo, I.F., Alston, C.L., Knowles, C., Taylor, R.W., McFarland, R., and Smeets, H.J. (2016). De novo mtDNA point mutations are common and have a low recurrence risk. In preperation.

4. Hauswirth, W.W., and Laipis, P.J. (1982). Mitochondrial DNA polymorphism in a maternal lineage of Holstein cows. Proceedings of the National Academy of Sciences of the United States of America 79, 4686-4690.

5. Cree, L.M., Samuels, D.C., de Sousa Lopes, S.C., Rajasimha, H.K., Wonnapinij, P., Mann, J.R., Dahl, H.H., and Chinnery, P.F. (2008). A reduction of mitochondrial DNA molecules during embryogenesis explains the rapid segregation of genotypes. Nature genetics 40, 249-254.

6. Sharpley, M.S., Marciniak, C., Eckel-Mahan, K., McManus, M., Crimi, M., Waymire, K., Lin, C.S., Masubuchi, S., Friend, N., Koike, M., et al. (2012). Heteroplasmy of mouse mtDNA is genetically unstable and results in altered behavior and cognition. Cell 151, 333-343.

7. Calvo, S.E., Clauser, K.R., and Mootha, V.K. (2016). MitoCarta2.0: an updated inventory of mammalian mitochondrial proteins. Nucleic acids research 44, D1251-1257.

8. Wonnapinij, P., Chinnery, P.F., and Samuels, D.C. (2008). The distribution of mitochondrial DNA heteroplasmy due to random genetic drift. American journal of human genetics 83, 582-593.

9. Stewart, J.B., Freyer, C., Elson, J.L., Wredenberg, A., Cansu, Z., Trifunovic, A., and Larsson, N.G. (2008). Strong purifying selection in transmission of mammalian mitochondrial DNA. PLoS biology 6, e10.

10. Brown, D.T., Samuels, D.C., Michael, E.M., Turnbull, D.M., and Chinnery, P.F. (2001). Random genetic drift determines the level of mutant mtDNA in human primary oocytes. American journal of human genetics $68,533-536$.

11. Thorburn, D.R. (2004). Mitochondrial disorders: prevalence, myths and advances. Journal of inherited metabolic disease 27, 349-362.

12. Uusimaa, J., Moilanen, J.S., Vainionpaa, L., Tapanainen, P., Lindholm, P., Nuutinen, M., Lopponen, T., Maki-Torkko, E., Rantala, H., and Majamaa, K. (2007). Prevalence, segregation, and phenotype of the mitochondrial DNA $3243 A>G$ mutation in children. Annals of neurology 62, 278-287.

13. Lee, H.S., Ma, H., Juanes, R.C., Tachibana, M., Sparman, M., Woodward, J., Ramsey, C., Xu, J., Kang, E.J., Amato, P., et al. (2012). Rapid mitochondrial DNA segregation in primate preimplantation embryos precedes somatic and germline bottleneck. Cell reports 1, 506-515.

14. Jenuth, J.P., Peterson, A.C., Fu, K., and Shoubridge, E.A. (1996). Random genetic drift in 
the female germline explains the rapid segregation of mammalian mitochondrial DNA. Nature genetics 14, 146-151.

15. Jenuth, J.P., Peterson, A.C., and Shoubridge, E.A. (1997). Tissue-specific selection for different mtDNA genotypes in heteroplasmic mice. Nature genetics 16, 93-95.

16. Wai, T., Teoli, D., and Shoubridge, E.A. (2008). The mitochondrial DNA genetic bottleneck results from replication of a subpopulation of genomes. Nature genetics 40, 1484-1488.

17. Arunachalam, M., Raja, M., Vijayakumar, C., Malaiammal, P., and Mayden, R.L. (2013). Natural history of zebrafish (Danio rerio) in India. Zebrafish 10, 1-14.

18. Kimmel, C.B., Ballard, W.W., Kimmel, S.R., Ullmann, B., and Schilling, T.F. (1995). Stages of embryonic development of the zebrafish. Developmental dynamics : an official publication of the American Association of Anatomists 203, 253-310.

19. Lieschke, G.J., and Currie, P.D. (2007). Animal models of human disease: zebrafish swim into view. Nature reviews Genetics 8, 353-367.

20. Santoriello, C., and Zon, L.I. (2012). Hooked! Modeling human disease in zebrafish. The Journal of clinical investigation 122, 2337-2343.

21. Howe, K., Clark, M.D., Torroja, C.F., Torrance, J., Berthelot, C., Muffato, M., Collins, J.E., Humphray, S., McLaren, K., Matthews, L., et al. (2013). The zebrafish reference genome sequence and its relationship to the human genome. Nature 496, 498-503.

22. Wolff, J.N., White, D.J., Woodhams, M., White, H.E., and Gemmell, N.J. (2011). The strength and timing of the mitochondrial bottleneck in salmon suggests a conserved mechanism in vertebrates. PloS one 6, e20522. 



\section{Chapter 2}

\section{Evolutionary origin of and}

\section{mechanisms in mitochondria}

Auke BC Otten, Hubert JM Smeets

First part of the publication 


\section{Introduction}

Mitochondria are fundamental to the origin of (multicellular) eukaryotes and provide the eukaryotes with the capability of generating energy, at the cost of high mutagenesis, partially due to the production of reactive oxygen species (ROS). It is this high mutation rate that has had a profound impact on the composition and inheritance of the genetic material within the mitochondria: the mtDNA. This mtDNA is indispensable for oxidative phosphorylation (OXPHOS), the process of aerobic ATP production in the mitochondria as it encodes part of the OXPHOS proteins (Figure 2.1). All other mitochondrial proteins are encoded by the nucleus and are imported into the mitochondria. The presence of ROS can lead to mutation rates which, in combination with uniparental, asexual inheritance, would eventually have led to disappearance of the mtDNA according to Muller's ratchet, but obviously that has not occurred. Eukaryotes have developed a variety of strategies to prevent fixation of mtDNA mutations. Although beneficial at the level of the species, these evolutionary mechanisms can have adverse effects at the level of the individual. The driving forces and underlying mechanisms of mitochondrial evolution across eukaryotes most likely explain how human mtDNA disease and mtDNA-related fertility problems arise and are being transmitted, and also reveal what approaches could be undertaken for treatment and/or prevention.

\section{Mitochondria: function, origin and evolution}

\section{Mitochondrial metabolism and oxidative phosphorylation (OXPHOS)}

Mitochondria are powerhouses producing the majority of cellular energy (Adenosine Triphosphate; ATP) inside the mitochondrial double membrane. The number of mitochondria within a cell can vary depending on the energy demand of the specific cell type. Although energy production is the main function of mitochondria, other cellular processes, such as heme synthesis, cellular apoptosis and calcium homeostasis, are also carried out by mitochondria.

Aerobic generation of energy is a multistep processes ending with oxidative phosphorylation (OXPHOS; Figure 2.1). NADH and $\mathrm{FADH}_{2}$ drive the OXPHOS system which is embedded in the mitochondrial inner-membrane and which consists of five enzyme complexes (I-V) and two electron carriers: coenzyme $Q$ and cytochrome $c$ (Figure 2.1). $\mathrm{NADH}$ and $\mathrm{FADH}_{2}$ donate electrons to and thereby reduce complex I (NADH) or complex II $\left(\mathrm{FADH}_{2}\right)$ which are re-oxidized by the electron carrier coenzyme $Q$. The chain of oxidation and reduction events and electron transfer is continued through complex III, electron carrier cytochrome $\mathrm{c}$ and complex IV. The energy released during this process is used by complex I, III and IV, but not II, to transport protons $\left(\mathrm{H}^{+}\right)$from the mitochondrial matrix into the inter-membrane space. Finally, these protons flow back across the membrane through complex $\mathrm{V}$, leading to the production of ATP out of ADP and inorganic phosphate (Pi). The ATP is transported out of the mitochondrial matrix into the cytosol by the adenine-nucleotide transporter (ANT1) ${ }^{1}$, where it can be used as an energy source (Figure 2.1). The process of OXPHOS is well-conserved, especially the core subunits ${ }^{2 ;}$, although some species lack complex I, e.g. most yeast ${ }^{4}$. 


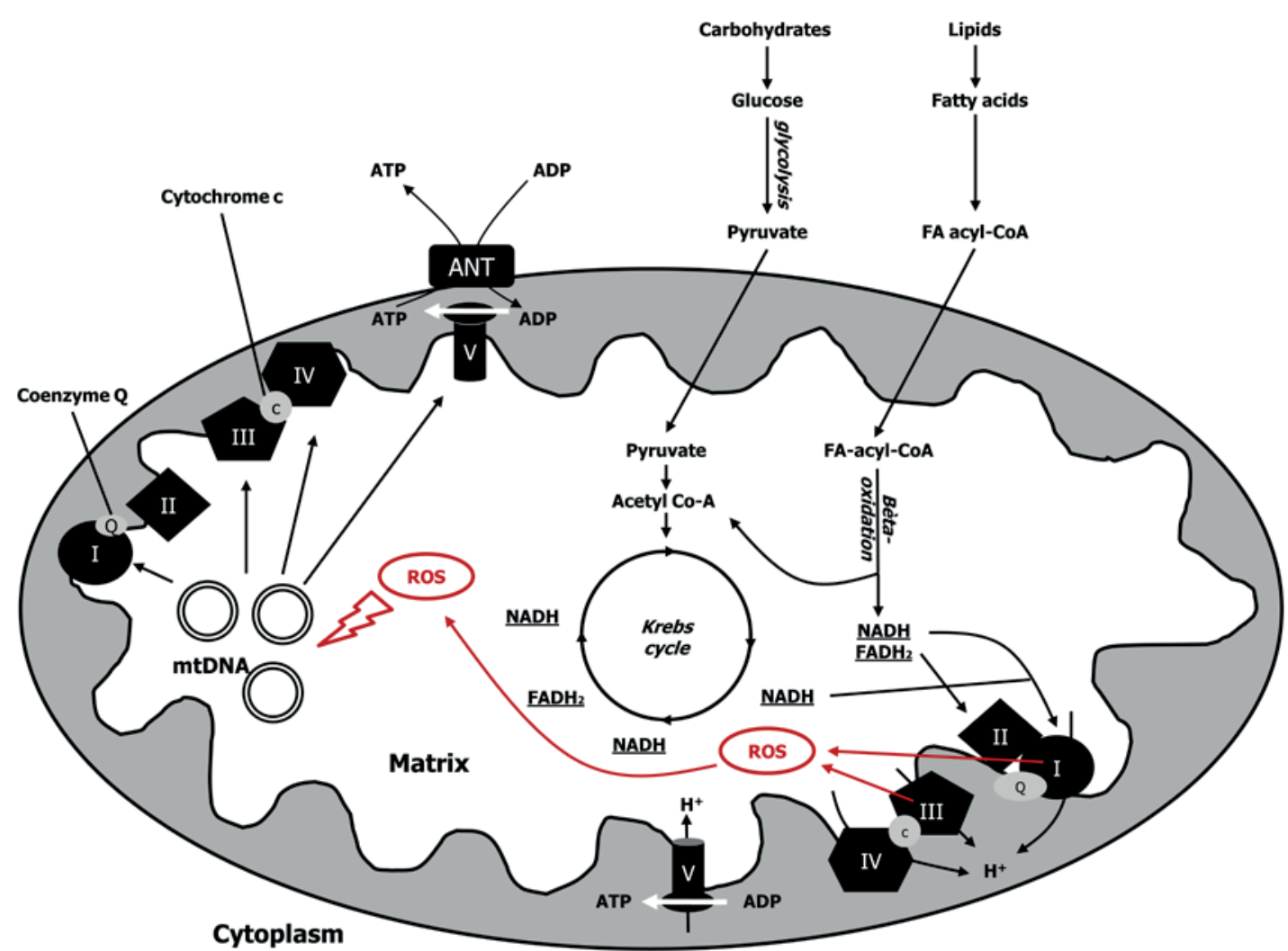

Figure 2.1 | Mitochondrial ATP production. ATP generation processes inside mitochondria and the production of ROS. Carbohydrates are broken down by glycolysis, resulting in ATP as well as pyruvate, which can be transformed into acetyl-CoA. Fatty acids (FAs) are biochemically dissimilated by mitochondrial beta-oxidation, which also results in acetyl-CoA. This acetyl-CoA is the substrate for the Krebs cycle, where the acetyl-group is donated to oxaloacetate, generating citric acid, which is dissimilated stepwise during the Krebs cycle resulting in ATP generation and production of the reducing agent $\mathrm{NADH}$. NADH is also produced during the beta-oxidation along with $\mathrm{FADH}_{2}$, another reducing agent. These reducing agents drive the process of ATP production by OXPHOS. ROS are produced as by-products of the OXPHOS and can damage the mtDNA.

\section{Evolution of mitochondria}

Originally, mitochondria were free-living prokaryotes but more than a billion years ago an endosymbiotic event made the free-living bacterium part of what became a eukaryotic cell ${ }^{5}$. It is unknown whether the symbiosis was immediately beneficial for the host. According to a recent theory, the mitochondrion originated from an obligate intracellular bacterium that parasitized on the energetic capabilities of its host due to its moderate OXPHOS capacity 6 . However, during evolution mitochondria changed from being a parasite into a central mutualistic organelle.

It has long been thought that the benefit for the anaerobic host, an amitochondrial eukaryote (or archezoan), was the acquired ability to detoxify oxygen ${ }^{7}$; this hypothesis is known as the archezoan theory ${ }^{8}$. However, the absence of amitochondrial 
eukaryotes $^{9}$ and early phylogenetic studies suggest that the archezoan theory is incorrect. Eukaryotic star-like-trees exist, composed of a few large hypothetical eukaryotic subgroups, each consisting of primarily microbial eukaryotes that radiated at the same time ${ }^{10}$. This fits with an alternative hypothesis, the symbiogenesis theory ${ }^{8}$, which, although the mechanism of endosymbiosis is unclear, suggests a prokaryotic rather than a eukaryotic host. Instead of detoxification of oxygen, hydrogen-dependent ATP production was the eventual fundamental force for the symbiotic association between an anaerobic (hydrogen dependent) archaebacterium (the host) and a respiring, facultative anaerobic eubacterium (the symbiont) that generated hydrogen as waste product ${ }^{11}$. The symbiogenesis theory is revolutionary, since it further implies that eukaryotes evolved integral, instead of prior, to mitochondrial endosymbiosis, as was generally accepted within the archezoan theory. The symbiogenesis theory also explains the ubiquitous and diverse nature of mitochondrial organelles ${ }^{7 ;}, 12$. The divergence of anaerobic mitochondria and mitochondria-related organelles, in combination with the widely accepted view that the symbiotic event occurred only once (since all known eukaryotic groups contain a preserved set of homologous genes in their mitochondrial DNA), can only be explained if the mitochondrial host was of anaerobic prokaryotic origin?.

Regardless of the founding theory, it is therefore without doubt that the mitochondrion itself is of prokaryotic origin. Prokaryotes lack a nucleus and organelles and their genetic material is soluble and located in the volume created by the cell membrane. Although the exact mitochondrial ancestor is unknown, a recent phylogenetic study has assigned this proto-mitochondrion to the class of alphaproteobacteria $^{13}$. Mitochondria are small compared to their alpha-bacterial ancestors and the Rickettsia species seems to be the closest relatives of mitochondria, with a genome of $1 \mathrm{Mb}$ (Table 2.1), containing all the homologous genes needed for OXPHOS ${ }^{14}$.

\section{Animal, plant and fungal mitochondrial genomes}

The mitochondrial genomes of animals, plants and fungi (Table 2.1) display a strong reduction in gene number and genome size during evolution. Most of these changes occurred between the bacterial ancestor and the Last Eukaryotic Common Ancestor (LECA) and are the result of both losses of alpha-proteobacterial genes, alongside with endosymbiotic gene transfer from the alpha-proteobacterial DNA to the nucleus. The net result is that mainly genes encoding proteins involved in energy metabolism and translation are maintained ${ }^{36}$, at the expense of, for example, genes for repair and recombination of DNA. Although most of these changes occurred very early during mitochondrial evolution, before the radiation of eukaryotes, subtle proteome evolution still occurs ${ }^{36}$ and, basically, the mtDNA is unique for each species and tailored for its specific nuclear background. As a result, mitochondria are not functional across species (further discussed under 'Human mitochondrial diseases'). 
Table 2.1 | Sizes and gene count of some pre-mitochondrial and mitochondrial genomes.

\begin{tabular}{|c|c|c|c|c|c|}
\hline & Genome & Size & $\begin{array}{r}\text { (Potential) } \\
\text { genes }\end{array}$ & Specific remarks & Ref. \\
\hline \multicolumn{6}{|l|}{ a-proteobacteria } \\
\hline B. japonicum & nuclear & 9,1 Mb & 8,317 & $43 \%$ similar to M. Loti & 15 \\
\hline M. loti & nuclear & $7 \mathrm{Mb}$ & 6,752 & & 15 \\
\hline C. crescentus & nuclear & $4 \mathrm{Mb}$ & 3,767 & & 16 \\
\hline R. prowazekii & nuclear & $1 \mathrm{Mb}$ & 834 & $\begin{array}{l}\text { all homologous genes } \\
\text { for the ATP system }\end{array}$ & $14 ; 17$ \\
\hline \multicolumn{6}{|c|}{ Early eukaryotes (animal relatives) } \\
\hline R. americana & $\mathrm{mt}$ & $69,034 \mathrm{bp}$ & 97 & $\begin{array}{l}\text { largest known mt gene } \\
\text { content }\end{array}$ & 18 \\
\hline M. brevicollis & $\mathrm{mt}$ & $76,568 \mathrm{bp}$ & 55 & & 19 \\
\hline A. parasiticum & $\mathrm{mt}$ & $\begin{array}{l}0.3-8.3 \\
\mathrm{kbp}\end{array}$ & 53 & $\begin{array}{l}\text { contains sets of mt } \\
\text { chromosomes }\end{array}$ & 19 \\
\hline \multicolumn{6}{|l|}{ Animals } \\
\hline C. elegans & $\mathrm{mt}$ & $13,764 \mathrm{bp}$ & 36 & lacks ATPase8 gene & 2 \\
\hline$X$. laevis & $\mathrm{mt}$ & $17,553 \mathrm{bp}$ & 37 & $\begin{array}{l}\text { Larger displacement } \\
\text { loop (D-loop) }\end{array}$ & 20 \\
\hline M. Musculus & $\mathrm{mt}$ & $16,299 \mathrm{bp}$ & 37 & & 21 \\
\hline B. taurus & $\mathrm{mt}$ & 16,338 bp & 37 & & 22 \\
\hline S. scrofa & $\mathrm{mt}$ & $16,613 \mathrm{bp}$ & 37 & & 23 \\
\hline H. sapiens & $\mathrm{mt}$ & $16,569 \mathrm{bp}$ & 37 & & 24 \\
\hline $\begin{array}{l}\text { O. } \\
\text { tshawytscha }\end{array}$ & $\mathrm{mt}$ & $16,644 \mathrm{bp}$ & 37 & & 25 \\
\hline D. rerio & $\mathrm{mt}$ & $16,569 \mathrm{bp}$ & 37 & & 26 \\
\hline \multicolumn{6}{|l|}{ Plants } \\
\hline M. viride & $\mathrm{mt}$ & $42,424 \mathrm{bp}$ & 65 & $\begin{array}{l}\text { a green algea (before } \\
\text { plant diversification) }\end{array}$ & 27 \\
\hline A. thaliana & $\mathrm{mt}$ & $\begin{array}{l}366,924 \\
b p\end{array}$ & 57 & & 28 \\
\hline M. polymorpha & $\mathrm{mt}$ & $\begin{array}{l}186,400 \\
\text { bp }\end{array}$ & 94 & & 29 \\
\hline $\begin{array}{l}\text { Curcubitaceae } \\
\text { sp. }\end{array}$ & $\mathrm{mt}$ & $2,400 \mathrm{kpb}$ & $?$ & & 30 \\
\hline \multicolumn{6}{|l|}{ Fungi } \\
\hline R. anserina & $\mathrm{mt}$ & $\begin{array}{l}100,314 \\
b p\end{array}$ & 81 & contains 16 introns & 31 \\
\hline S. commune & $\mathrm{mt}$ & $49,704 \mathrm{bp}$ & 49 & $\begin{array}{l}\text { contains no introns, no } \\
\text { repeat sequences }\end{array}$ & 32 \\
\hline S. cervisae & $\mathrm{mt}$ & $85,779 \mathrm{bp}$ & 46 & & 33 \\
\hline
\end{tabular}




\begin{tabular}{|l|l|l|l|r|l|r|}
\hline H. uvarum & $\mathrm{mt}$ & $18,884 \mathrm{bp}$ & 33 & $\begin{array}{l}\text { shortest fungal mito- } \\
\text { chondrial genome, } \\
\text { linear }\end{array}$ & 34 \\
\cline { 2 - 6 } & C. alblicans & $\mathrm{mt}$ & $40,420 \mathrm{bp}$ & 45 & $\begin{array}{l}\text { almost no recombina- } \\
\text { tion }\end{array}$ & 35 \\
\hline
\end{tabular}

The mtDNA has a genetic code, which differs from the genetic code in the nucleus (NCBI: http://www.ncbi.nlm.nih.gov/Taxonomy/Utils/wprintgc.cgi). These differences arose during evolution after endosymbiosis. For example, in metazoan mitochondria the UGA codon is allocated to Tryptophan, while it serves a stop-codon in the nucleus, indicating that this codon reassignment has occurred within a common ancestor of Metazoa. Likewise, the AGN, AUN and AAN codons changed during metazoan evolution. The characteristics of the transfer RNAs (tRNAs) restrict the manner in which codes can change ${ }^{37}$ and it seems that endosymbiotic gene transfer continued until, although roughly estimated, the UGA codon was switched from a stop codon to amino acid coding in the animal and fungal line ${ }^{38 ; 39}$. A number of proteins from alpha-proteobacterial origin were lost and replaced by host proteins as part of the process of non-orthologous gene displacement ${ }^{40}$, giving some species special types of proteins that are absent in others, depending on when and how the event occurred during evolution.

Crucial in the development of mitochondria as intracellular organelles was the acquisition of a system to import proteins from the cytosol into the mitochondria ${ }^{41}$. This system facilitated both expansion of the mitochondrial proteome and mtDNA genome reduction, since nuclear-encoded proteins can be imported into the mitochondria (Gabaldon and Huynen, 2004). Acquisition of the ATP/ADP transporter to release mitochondrial-produced ATP into the cytosol gave the mitochondria their crucial position as powerhouses ${ }^{42}$. Together, gene loss, replacement, transfer and acquisition have made modern mitochondria separate from their alpha-proteobacterial ancestors.

\section{Animals}

Animal mitochondrial genomes have some important unique characteristics. First, the mtDNA underwent the most extensive genome reduction, resulting in a compact genome, ranging from 13,764 bp in c. elegans to 17,553 bp in $x$. laevis (Table 2.1), with only $14-16 \%$ of the proto-mitochondrial (apha-bacterial) proteome still present ${ }^{2}$. Some structural OXPHOS subunits and the necessary protein synthesis genes remained in the mtDNA in higher animals, whereas the other genes were either transferred to the nucleus or lost due to the presence of genes with similar function in the nuclear genome (Adams and Palmer, 2003). In almost all animal species, including humans, the mtDNA contains only 37 genes, of which 13 are protein-coding genes, 2 encode ribosomal RNAs (rRNA) and 22 encode transfer-RNAs (tRNA) ${ }^{43}$ (Table 2.1). Since tRNAs cannot be imported from the cytosol into the mitochondria, the tRNAs needed to translate the (different) genetic code are encoded by the mtDNA itself ${ }^{37}$. Secondly, animal mitochondrial genomes contain no introns and few repeat sequences ${ }^{43}$. As a result, animal mtDNAs show only limited signs of recombination ${ }^{44}$. Thirdly, animals maintain a high mtDNA copy number (Table 2.2). A cell can contain over 500-1,000 mtDNA molecules, depending on the energy demand of a cell ${ }^{45}$. In oocytes, the 
mtDNA copy number is highest, ranging from 100,000 to $14,000,000$ copies. A fourth feature of animal mitochondrial genomes is its exclusive maternal inheritance, as sperm mitochondria are actively degraded ${ }^{46}$ by proteasomal (ubiquitin) and lysosomal (mitophagy) degradation pathways, during which ubiquitin-binding receptors might serve as mitophagy receptors; this mechanism is most likely prevalent amongst the animal taxa (extensively reviewed in 47; 48 ). Finally, gene replacement and transfer have led to several genes being unique to the animals (Metazoa) or to animals and fungi (Ophistokonta) (see Table 2.1 from ${ }^{36}$ ), an important one being the nuclearencoded DNA polymerase gamma genes (POLG and POLG2), which have replaced the bacterial genes $d n a N, d n a E$ en $d n a Q$. POLG is the only polymerase found in mammalian mitochondria and is of viral origin due to gene exchange ${ }^{49}$.

Table 2.2 | Features of the mitochondria and mtDNA in animals, fungi and land plants

\begin{tabular}{|l|c|c|c|}
\hline & Animals & Fungi & Land plants \\
\hline Compaction of mtDNA & + & $+/-$ & - \\
\hline Average number of genes & $36-37$ & $30-80$ & $50-100$ \\
\hline Gene order conservation & + & - & $+/-$ \\
\hline mtDNA size (typical) & $13-17 \mathrm{~kb}$ & $20-100 \mathrm{~kb}$ & $180-400 \mathrm{~kb}$ \\
\hline $\begin{array}{l}\text { Introns, repeat elements and } \\
\text { recombination }\end{array}$ & - & + & + \\
\hline Copy number & High & Intermediate? & Low \\
\hline Type of inheritance & Maternal & Paternal, maternal \\
and biparental & Maternal \\
\hline Antioxidant system & $+/-$ & $+/-$ & + \\
\hline
\end{tabular}

\section{Plants}

Plant mitochondria differ from their animal counterparts in a number of ways. Firstly, genome reduction was less pronounced and more variable in the plant mtDNA, which can contain over 100 genes $^{28}$. In addition, several genes that were transferred during animal evolution are retained in plant species, like the complex II genes sdh3 and $s d h 4^{27 ;} 28$. Especially the mtDNA size of land plants differs from animal mtDNA, ranging, on average, from $\sim 180-370 \mathrm{~kb}$, with some extreme exceptions (Table 2.1). Non-coding DNA was inserted in the mtDNA after the evolutionary branching of the land plants, since the common ancestor was gene rich and contained little introns ${ }^{50}$ and this resulted in the presence of repeated segments, introns, intronic Open Reading Frames (ORFs), and incorporated foreign DNA of plastid, nuclear or plasmid origin ${ }^{28 ;}$ 51 . With all these features, the mtDNA genomes of plants resemble, unlike their animal counterparts, the size-relaxed nature of a nuclear genome, including recombination events, which give plants the ability to possess sub-genomic molecules variable in 
size $^{52}$. Therefore, no universal mtDNA copy number exists in plants, but there are differential gene copy numbers, as assessed by four mitochondrial genes that differ in their copy number from each other and amongst tissues in the Arabidopsis species ${ }^{52}$. Similar to animals, the predominant mode of mitochondrial inheritance is maternal ${ }^{44}$, governed by a variety of mechanisms, including methylation and/or zygote-specific gene expression ${ }^{47}$.

Important for plants is the presence of another endosymbiont, the chloroplasts. Its key function, photosynthesis, generates carbohydrates and ATP from sunlight and water, which is the main energy source for chloroplast-containing organisms, while mitochondria are the main energy source (due to respiration) for organisms without chloroplast. Mitochondria are still indispensable in plants for chloroplast function and provide an alternative respiratory pathway for metabolic flexibility during stress (e.g. high ROS, cold, drought ${ }^{53}$ ). Alternative oxidase (AOX) in plant mitochondria is then upregulated, to circumvent complex III and IV of the oxidative phosphorylation, accepting carbons directly from the TCA cycle ${ }^{54}$, thereby reducing ROS damage. Furthermore, plants harbour two non-proton-pumping $\mathrm{NAD}(\mathrm{P}) \mathrm{H}$ dehydrogenases, which are activated during stress ${ }^{55 ;} 56$. Finally, in plants the nuclear-encoded MSH1 gene is a unique property of the species, since it is the result of the fusion between a bacterial DNA-repair homologue of MutS and an endonuclease. It seems that this gene has enabled plants to have recombination-mediated repair of the mtDNA ${ }^{57}$. As in animals, acquisition of genes can be restricted to the clade of plants.

\section{Fungi}

Animal and plant mitochondria have been studied extensively, while their fungal counterparts are much less-well described. Although fungi are evolutionary more close to animals than plants, their mtDNA contains many plant characteristics. The presence of introns, which can be up to $5 \mathrm{~kb}$ in length ${ }^{58}$, explains the differential size seen in fungal mtDNA, which ranges on average from 20-100 kbp, with some large exceptions (Table 1). The appearance of repetitive elements in introns give fungal mtDNAs their recombination capabilities ${ }^{58}$, which partly explains the enormous variation in gene order among fungal mtDNAs ${ }^{58-60}$. It is not clear whether fungi have a mitochondrial DNA copy number comparable to animals. However, since recombination is present, it is expected that their copy number can be regulated in a similar fashion as in plants and that sub-genomic mtDNA molecules will exist. Fungi are less complex eukaryotes with no strict energy requirements and, although they contain no chloroplasts, fungi are not as dependent on carbohydrates and lipids as animals. Instead, fungi display a high degree of versatility in their metabolic demands and also use different organic substrates, which can be as simple as nitrate or ethanol. Fungi are scavengers and feed on nutrient molecules from others and secrete digestive enzymes to the exterior to digest any nutrient that is available. The net result is that fungi are less dependent on mitochondria than are animals, but more dependent than are plants due to the absence of chloroplasts.

The mtDNA inheritance of fungal mitochondria is complex, with no universal mechanism of inheritance, allowing both uniparental and biparental inheritance ${ }^{44}$. In yeast, an almost equal contribution of mitochondria from both parents contributes to the zygote ${ }^{61}$. Some fungi have biparental inheritance of mitochondrial plasmids (small extragenomic mtDNA molecules) that retain parental mtDNAs ${ }^{62}$. Fungal 
mitochondria are considered as somewhere in between the evolution of plant and animal mitochondria.

\section{Summary}

Although most mtDNA changes appear to have taken place before the radiation of the eukaryotes, the evolutionary paths of mtDNA among the main eukaryotic clades could not differ more (Table 2.2). In the next part of this chapter, we will focus on how these differences have evolved and what the impact is on mitochondrial biology for humans in health and disease.

\section{Evolutionary mechanisms in mitochondria}

\section{Mutations in the mtDNA: Muller's ratchet and mutational meltdown}

Asexual populations accumulate detrimental mutations according to Muller's ratchet $^{63-65}$, because the accumulation is irreversible due to the absence of mutationrecovering mechanisms, such as sexual reproduction and recombination-mediated repair, eventually decreasing the fitness of a population ${ }^{64 ; 66-68}$. Extensive modelling ${ }^{69}$ and laboratory experiments provide evidence for the existence of Muller's ratchet and its potential for decreasing fitness by a mutational meltdown ${ }^{64}$. Muller's ratchet explains, for example, the high incidences of non-synonymous mutations in Caenorhabditis briggsae $^{70}$ and the degeneration of the neo-Y-chromosome in Drosophila miranda ${ }^{71}$. Such degeneration has also been discussed for the mammalian Y-chromosome ${ }^{72}$.

\section{Muller's ratchet in mtDNA}

The mtDNA is an asexual population, being inherited maternally in animals and plants, making this genome also prone to the detrimental consequences of Muller's ratchet. Following the path of evolution, the endosymbiosis of the mitochondria allowed an increased level of cellular energy and the expression of a substantially larger number of proteins in eukaryotes than in prokaryotes ${ }^{5 ; 73}$. This increased energy is produced by OXPHOS, during which electrons leak to $\mathrm{O}_{2}$ and produce reactive oxygen species (ROS), like superoxide ${ }^{74}$. This ROS toxicity arose simultaneously with the symbiosis event and the integration of metabolic pathways even enhanced the ROS production, that originally served as a cellular response at the outskirts of the (possibly parasitic) bacterium ${ }^{75}$, also observed during plant-pathogen interactions ${ }^{76}$ and in infected HeLa cells ${ }^{77}$. While unicellular eukaryotes have low energy consumption and subsequent less ROS production, the symbiosis event and the development of multicellularity brought the ROS-producing endosymbiont inside the eukaryotic cells, exposing the newborn eukaryotic (multicellular) organisms to a high and detrimental ROSinduced mutagenesis level. This increased mutation pressure, in combination with the limited action of recombination, e.g. in mice ${ }^{78}$ and humans ${ }^{79}$, implies an increased vulnerability of mtDNA to a mutational meltdown. Indeed, in mammals, the mutation rate in mitochondria is much higher than in the (recombining) nucleus ${ }^{80}$. Muller's 
ratchet in mitochondria has been quantified ${ }^{81}$ and molecular proof exists for the process in mitochondrial transfer RNAs, which accumulate mutations more rapidly compared to their nuclear counterparts ${ }^{80}$.

If Muller's ratchet could not be counteracted, the inevatible consequence of its genomic decay is extinction of species. However, mtDNA and eukaryotes are still present today, suggesting solutions for preventing detrimental mutation accumulation and subsequent declines in reproductive fitness, which, in the context of mtDNA, are specific mechanisms.

\section{Mitochondrial evolutionary mechanisms to balance high mutation loads}

One of the parameters determining the rate of mutation-related decline in fitness is the rate at which these mutations are introduced. In animals, this mutation rate is much higher (up to $100 x$ in mammals) compared to that in plants. In mammalian mitochondria, the mutation rate is $\sim 25 x$ higher than in the nucleus, whereas in plants this difference is much lower ${ }^{67}$. Fungal mutation rates are more similar to plants. A higher mutation rate in the mtDNA in animals might reflect a higher adaptive capacity to balance mitochondrial energy and heat requirements with respect to the environment ${ }^{82}$. Plants lack this adaptive need, since they typically live in restricted ecological niches, can rely on both mitochondria and chloroplasts for their energetic needs and they can survive as seeds, which animals cannot. Fungi share this feature of plants by the production of spores and are also less dependent on mitochondria due to their metabolic versatility. A higher mutational rate will generate mutations of any type, meaning that higher adaptive capacities are at the cost of a higher detrimental mutation load and a higher risk for mutation accumulation.

Several mechanisms have evolved to prohibit mutation accumulation: mutation prevention, diluting the chance of acquiring mutations, and recovery of mutations (Table 2.3). Where endosymbiotic gene transfer (EGT) was an effective general mechanism to lower mutation accumulative effects, species-specific mechanisms also exist, underlying differences in mutation rates, as well as differences in the mtDNA composition among species.

\section{Mutation prevention: endosymbiotic gene transfer and antioxidants}

As mentioned above, a general way to avoid mutation accumulation is moving the mtDNA genes to the nucleus, which is achieved by EGT, which had already occurred in the LECA. Changes in the mtDNA genome from this point resulted in the specific characteristics of modern mitochondria in different species. The early evolutionary pressure was likely to be very high, since EGT occurred at high rates in all eukaryotic species $^{83}$, despite being very complex and involving, for example, acquisition of a transit-peptide to re-access the mitochondrion. This is corroborated by the fission of several mitochondrial proteins, indicating that even the transfer of only a small part of a gene is favoured, as illustrated for the hydrophilic c-terminal portion of cytochrome c oxidase subunit 1 gene (COX1; a subunit of complex IV) ${ }^{84}$. It is conceivable that gene redundancy together with mitochondrial ROS-mediated high mutations rates drive EGT. Since the nucleus is less prone to mutations, because of its protection by histones and extensive repair and recombination, EGT is a successful tool to reduce mutation loads. The higher degree of EGT observed in animals, might therefore be 
a reflection of the higher mutation rate of animal mitochondria. However, EGT was not completed and mtDNA has remained in all species, most likely for biochemical reasons (the combination of extreme hydrophobicity of the mtDNA-encoded proteins, precluded easy import in the mitochondria) and the introduction of differences in the genetic code (as with the UGA codon) ${ }^{38}$. Mutation accumulation risk might not be the only evolutionary force that contributed to EGT. Selection for small organellar genomes might also be involved, having a replicative advantage, as the mtDNA size is linked to the time and energy that is needed to complete mitochondrial duplication ${ }^{85}$, and the level of metabolic efficiency ${ }^{86}$.

The evolutionary role of ROS is obvious from the evolvement of additional eukaryotic mitochondrial capabilities to mitigate the disadvantages of ROS production. These include 1) the peroxisomal breakdown of fatty acids (FAs) which normally render a lot of ROS, 2) the uncoupling of protons without the formation of ATP and ROS, 3) a shuttle that ensures high concentrations of the antioxidant carnitine and 4) the breakdown of dysfunctional ROS-producing mitochondria by mitophagy-mediated mitochondrial quality control $75 ; 87 ; 88$. Mutation rates introduced by ROS can be further lowered by the presence of antioxidants. Especially in plants and fungi, these systems, such as the AOX-system during stress conditions in plants, are upregulated so that they scavenge most of the ROS that is produced.

\section{Diluting the target: high copy number or introduction of non-coding DNA}

Plants and fungi have incorporated stretches of DNA that allow recombinationmediated mtDNA repair (a feature that is almost absent in animals) so proteincoding parts are less likely affected. Animals achieve this by maintaining a multi-copy genome (typically > 500 copies / animal cell). A high copy number ensures that not all genes can be affected by a mutation at the same time, leaving fully functional sets of proteins always present. Mammalian mtDNA copy numbers are highest in oocytes, typically above 100,000 copies, but vary among different animal species (Table 2.4). There seem to be three classes: in the first class, represented by humans, mice and rats, oocytes can have an mtDNA copy number between 100,000 and 200,000 copies, although some outliers are found. Second, for example in bovines, sheep and pigs, the mtDNA counts of oocytes are typically above 300,000 , but below $1,000,000$ copies. The final class, represented by salmon and zebrafish, have the highest mtDNA copy numbers of several millions genomes.

An explanation for this variation might be the different implantation patterns among the species or even the lack of implantation. Implantation has been classified into three categories: centric, eccentric and interstitial ${ }^{89}$, based on the different types of interactions between blastocysts and uterine cells. Centric implantation is characterized by a large expansion of the blastocyst before implantation, followed by fusion with the luminal epithelium ${ }^{90}$. Bovines, sheep and pigs have this type of implantation. Mice and rats, on the other hand, have an eccentric implantation pattern, where the luminal epithelium forms an invagination to surround the trophoblast. The human implantation pattern is interstitial as the trophoblast passes through the luminal epithelium to invade the endometrial stromal and become imbedded into the wall of the uterus ${ }^{89}$. A fourth group consisting of for example salmon and zebrafish, have no implantation at all. Another factor can be the speed at which implantation occurs.

Rats, mice and humans have an immediate, fast implantation, with immediate access 
to high levels of nutrients within the female reproductive tract that can be utilized by the embryo ${ }^{91}$, whereas at the other end of the spectrum, as in salmon and zebrafish, implantation is absent. The class with bovines, sheep and pigs are in between with a more delayed implantation. A high copy number in oocytes is needed to distribute mtDNA molecules over the daughter cells in the absence of mtDNA replication during early embryogenesis ${ }^{92 ; 93}$ and the differences in copy number between species could be, although speculative, a reflection of different energetic needs during early development in animals. These energy needs range depending on whether there is a rapid direct access to the maternal blood supply, as in humans (lowest mtDNA copy number), delayed access (intermediate mtDNA copy number), or a complete dependence on their own energy storage from the yolk-sac during the whole of embryogenesis ${ }^{94}$, as in fish (highest mtDNA copy number). The longer implantation is delayed, the higher the energy demand is for the pre-implantation embryo, being most extreme when implantation is completely absent (as in most cold-blooded species).

\section{Mutation recovery: recombination-mediated repair}

The last class of evolutionary mechanisms aims to remove or repair mtDNA mutations. For this, plant mtDNA undergoes recombination, mediated by repeat sequences, while animal mtDNA does not or has only limited recombination. Repeat sequences cause ectopic recombination, a potential harmful feature that can disrupt coding frames and gene expression, generating an additional selective pressure for efficient mtDNA repair. For this repair, plants harbor a unique fusion-product: the nuclear-encoded $\mathrm{MSH}_{1}$ gene $^{57}$. In Arabidopsis, the MSH1 gene allows plants to have an efficient recombination-mediated mismatch repair and gene conversion, which reduces heteroplasmy ${ }^{95}$ and is driven by the presence of repeat sequences ${ }^{96}$. Fungal MSH1 genes are homologues of the MutS gene, but do not possess the endonuclease domain and have been reported not to be involved in mismatch repair in yeast ${ }^{97}$. Although fungi display recombination due to accumulated repeats and introns, resulting in a high variability in the mitochondrial gene order, their mutation rate is intermediate between animals and plants ${ }^{59 ; 67}$. Recombination-mediated mtDNA repair in plants and some fungi can also occur via other mechanisms (see ${ }^{98}$ ) and is a perfect tool for lowering the ROS-generated mutational burden (further discussed in: $\left.{ }^{99 ; 100}\right)$. 
Table 2.3 | Mechanisms to reduce ROS-induced mutagenesis in animals, fungi and land plants after their evolutionary diversification

\begin{tabular}{|c|c|c|c|}
\hline Prevention of & \multicolumn{3}{|c|}{ Endosymbiotic Gene Transfer (EGT) } \\
\hline $\begin{array}{l}\text { Species-specific } \\
\text { mechanisms }\end{array}$ & Animals & (Land) plants & Fungi \\
\hline $\begin{array}{l}\text { Prevention of } \\
\text { mutations }\end{array}$ & $\begin{array}{ll}- & \text { Antioxidants } \\
\text { (only limited) }\end{array}$ & $\begin{array}{l}\text { - Highly developed } \\
\text { antioxidants } \\
\text { - Chloroplast } \\
\text { dependency }\end{array}$ & $\begin{array}{ll}\text { - } & \text { Moderate } \\
\text { antioxidants } \\
\text { - } \\
\text { Metabolic } \\
\text { Diversity }\end{array}$ \\
\hline $\begin{array}{l}\text { Dilution of mutational } \\
\text { chance }\end{array}$ & $\begin{array}{l}\text { mtDNA copy } \\
\text { number com- } \\
\text { bined with a } \\
\text { small genome }\end{array}$ & $\begin{array}{l}\text { - Large genomes } \\
\text { with introns }\end{array}$ & $\begin{array}{ll} & \text { Large } \\
\text { genome with } \\
\text { introns }\end{array}$ \\
\hline $\begin{array}{l}\text { Recovery of } \\
\text { mutations }\end{array}$ & $\begin{array}{ll}\text { - } & \text { mtDNA } \\
\text { bottleneck } \\
\text { - } \\
\text { Purifying } \\
\text { selection }\end{array}$ & $\begin{array}{l}\text { Uptake of } \\
\text { elements to allow } \\
\text { recombination } \\
\text { and mtDNA repair }\end{array}$ & $\begin{array}{ll}\text { - } & \text { Uptake of } \\
\text { elements to } \\
\text { allow recom- } \\
\text { bination and } \\
\text { mtDNA repair } \\
\text { - } \quad \text { Bottleneck } \\
\text { - } & \text { Somatic } \\
& \text { Fusion }\end{array}$ \\
\hline
\end{tabular}

\section{Mutation recovery: the mtDNA bottleneck}

During the cleavage stages of embryogenesis, the total amount of mtDNA is constant, indicating a random partitioning of the mtDNA over the different daughter cells ${ }^{101 ; 102}$ and only those cells that develop into an embryo will contribute to the mtDNA pool of the new-born embryo, leading to a strong numerical bottleneck ${ }^{103}$. In 1989 , such a bottleneck for mtDNA inheritance was first observed in Holstein cows, resulting in rapid fixation of heteroplasmic variants during transmission to following generations and a return to homoplasmy ${ }^{104}$; this implies a sampling effect of mtDNA molecules early during embryogenesis. A restriction in the number of mtDNA molecules to be transmitted to the next generation is followed by a strong amplification of these founder molecules. During oogenesis in mice, a 100-fold increase in the mtDNA amount has been observed, without any further increase during preimplantation development ${ }^{105}$. The sampling effect and random genetic drift, during the formation of primordial germ cells (PGCs) and oocyte maturation (clonal expansion) determine the mtDNA that is inherited. The net result is a reset to homoplasmy within a very short time-frame in which germ-cell mtDNA is most sensitive to mutagenesis.

Besides the observations in mammals and rodents, the mtDNA bottleneck has been described in Chinook salmon ${ }^{106}$ and Danio rerio (See chapter 3 ). These two teleost species are non-mammalian vertebrates and both show an mtDNA bottleneck that is remarkably similar to the one documented in mice. This indicates that, within the clade of Metazoa or at least the Vertebrata, the mitochondrial DNA bottleneck is a highly conserved mechanism. The mtDNA copy number is brought back to $\sim 200$ 
in germ cells, which seems quite conserved in humans, mice ${ }^{102 ; 107}$, salmon ${ }^{106}$ and zebrafish (See chapter 3). Apparently, this amount of mtDNA protects the species from pre-existing mutation accumulation due to rapid segregation ${ }^{108}$, while preserving fertility, resulting in a low vulnerability for de-novo mutations to reach significant heteroplasmy levels (as discussed below).

\section{Mutation recovery: bottleneck-mediated selection}

Mutations that escape the mtDNA bottleneck can be filtered out by purifying selection at the level of the organelle in animals during early embryonic development ${ }^{103}$; 109. Purifying selection can take place either by positive or negative selection on mtDNA sequences and evidence exists for both processes ${ }^{110 ; 111}$. In the mutator mouse, mutations accumulate at random in the mtDNA and purifying selection has been detected by the analysis of mutation distribution after backcrossing. Potential deleterious mutations are strongly underrepresented, implying selection against these mutations in the mammalian germ line ${ }^{109}$. Furthermore, in transmitochondrial mice, a severe OXPHOS-affecting mutation is eliminated rapidly from the maternal germ line ${ }^{112}$, further proving the existence of purifying selection. The exact mechanism of purifying selection at the level of the organelle is unknown, but is thought to be achieved by functional testing of mtDNA at the single organelle level, most likely near or at the bottom of the bottleneck. A number of mechanisms have been proposed ${ }^{113}$. The absence of mtDNA replication during early embryogenesis ${ }^{114}$ leads to a very low mtDNA count in the primordial germ cells. Most likely, this is a prerequisite to selectively filter out deleterious mtDNA mutations, since it is only when the mtDNA load is small, that differences in organelle fitness can manifest quickly and be selected for ${ }^{115}$. Another possible mechanism of purifying selection has been postulated for Drosophila melanogaster ${ }^{116}$, based on selective replication of wild-type mtDNA molecules with a more robust OXPHOS capacity; this hypothesis is enforced by the observation that selective replication of functional mtDNA molecules is able to restrict the transmission of deleterious mtDNA variants and that the replicative advantage is coupled to mitochondrial fitness ${ }^{117}$. The exact nature of this replicative advantage is not known, but could be achieved by the energy demanding burst of mtDNA replication during oogenesis, allowing selection for mtDNA with an efficient energy production, thus favouring their replication. Finally, mtDNA correction based on fusion and fission of mitochondria could be implicated in purifying selection, leading to selective breakdown (mitophagy) of damaged mitochondria ${ }^{118}$; this process is thought to be regulated by Parkin ${ }^{119}$. However, purifying selection seems to occur independently of Parkin in D. Melanogaster ${ }^{116}$. 


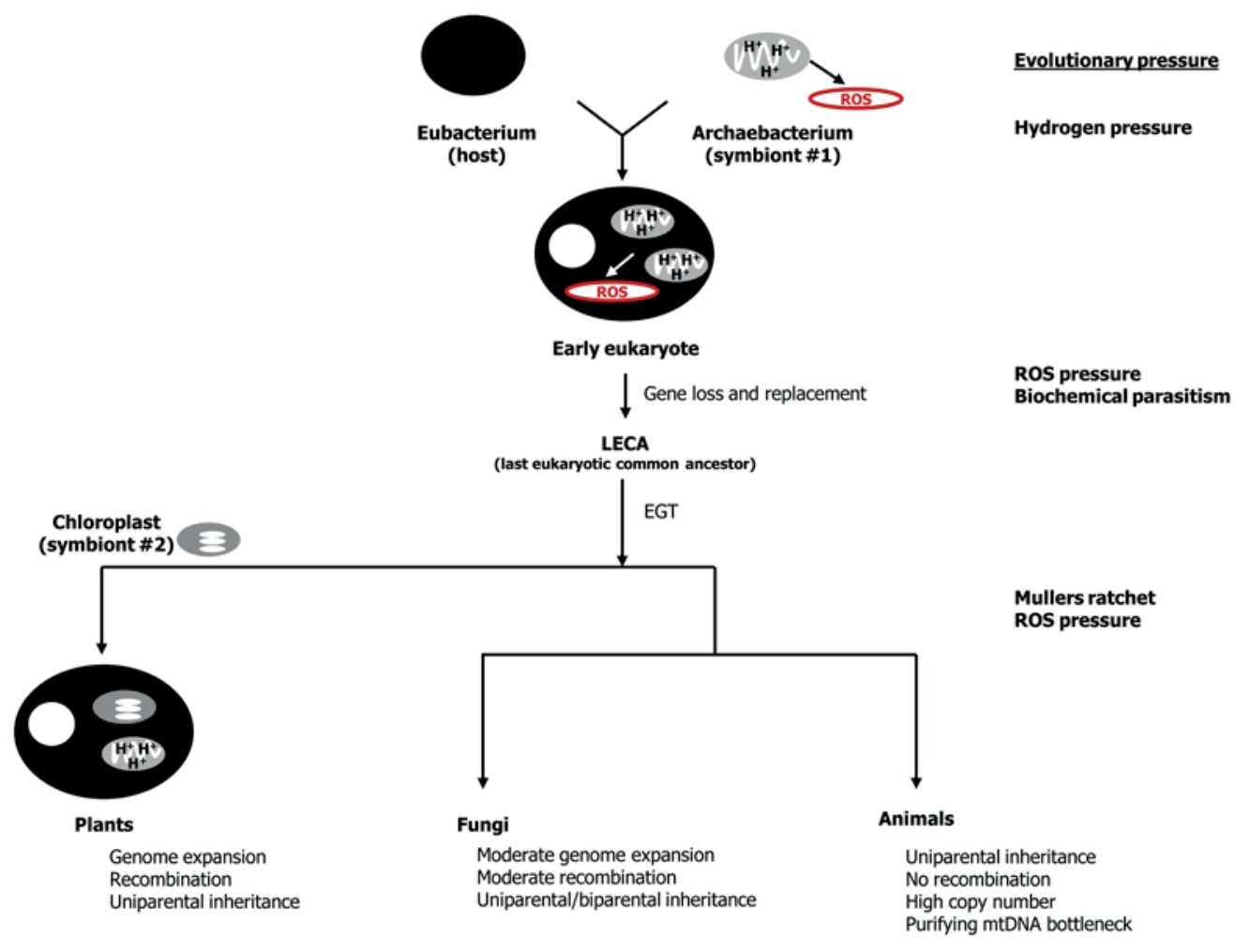

Figure 2.2 | Mitochondrial evolution and eukaryogenesis. An early endosymbiosis between two prokaryotes led to the eukaryotes, which have evolved differently with regard to their mitochondrial DNA.

\section{Summary}

As shown in Table 2.3 and Figure 2.2, animals, plants and fungi have, besides EGT, different species-specific mechanisms to ensure mitochondrial sequence homoplasmy and to slow mutation accumulation. The need for higher adaptive abilities enforced animals to allow higher mutation rates, and the detrimental consequences are counteracted by the mtDNA bottleneck and purifying selection. The result for the species is an optimal balance between negative deleterious mutations and positive adaptive mutations. As the bottleneck affects reproductive fitness, this is also a critical factor in this balance. The incorporation of repeat elements in the mtDNA of plants has enabled efficient recombination-mediated mtDNA repair in plant mtDNA, which results in low mitochondrial mutation rates. Fungi are described to have bottlenecks and restricted somatic fusions ${ }^{120}$, while they simultaneously possess recombinationmediated mtDNA repair mechanisms ${ }^{59}$. 
Table 2.4 | mtDNA copy numbers in oocytes amongst different animal species

\begin{tabular}{|c|c|c|c|}
\hline Species & Median & Copy number oocytes & Reference \\
\hline \multirow[t]{7}{*}{ H. Sapiens } & 256,000 & 143,000 & 121 \\
\hline & & 163,698 & 122 \\
\hline & & 193,000 & 123 \\
\hline & & 256,000 & 124 \\
\hline & & 384,251 & 125 \\
\hline & & 598,350 & 126 \\
\hline & & 697,176 & 127 \\
\hline \multirow[t]{8}{*}{ M. Musculus } & 243,500 & 131,990 & 128 \\
\hline & & 157,000 & 101 \\
\hline & & 175,000 & 107 \\
\hline & & 238,000 & 129 \\
\hline & & 249,000 & 102 \\
\hline & & 269,386 & 93 \\
\hline & & 430,000 & 130 \\
\hline & & 430,000 & 131 \\
\hline \multirow[t]{2}{*}{ R. Norvegicus } & 128,800 & 110,000 & 132 \\
\hline & & 147,600 & 133 \\
\hline \multirow[t]{7}{*}{ S. scrofa } & 246,984 & 138,022 & 134 \\
\hline & & $145,368-161,787$ & 135 \\
\hline & & $239,392-275,132$ & 136 \\
\hline & & 246,984 & 137 \\
\hline & & 285,000 & 138 \\
\hline & & $323,038-503,263$ & 139 \\
\hline & & 347,023 & 140 \\
\hline \multirow[t]{6}{*}{ B. taurus } & 367,057 & $134,896-204,173$ & 138 \\
\hline & & 260,000 & 141 \\
\hline & & 361,113 & 142 \\
\hline & & 373,000 & 92 \\
\hline & & 385,332 & 143 \\
\hline & & 807,794 & 144 \\
\hline O. aries & 744,633 & 744,633 & 145 \\
\hline O. tshawytscha & $3,200,000$ & $3,200,000$ & 106 \\
\hline \multirow[t]{2}{*}{ D. rerio } & $16,500,000$ & $19,000,000$ & Chapter 3 \\
\hline & & $14,000,000$ & 146 \\
\hline
\end{tabular}




\section{References}

1. Neckelmann, N., Li, K., Wade, R.P., Shuster, R., and Wallace, D.C. (1987). cDNA sequence of a human skeletal muscle ADP/ATP translocator: lack of a leader peptide, divergence from a fibroblast translocator cDNA, and coevolution with mitochondrial DNA genes. Proceedings of the National Academy of Sciences of the United States of America 84, 7580-7584.

2. Gabaldon, T., and Huynen, M.A. (2003). Reconstruction of the proto-mitochondrial metabolism. Science 301, 609.

3. Gabaldon, T., and Huynen, M.A. (2004). Shaping the mitochondrial proteome. Biochimica et biophysica acta 1659, 212-220.

4. Baile, M.G., and Claypool, S.M. (2013). The power of yeast to model diseases of the powerhouse of the cell. Frontiers in bioscience 18, 241-278.

5. Lane, N., and Martin, W. (2010). The energetics of genome complexity. Nature 467, 929-934.

6. Wang, Z., and Wu, M. (2014). Phylogenomic reconstruction indicates mitochondrial ancestor was an energy parasite. PloS one 9, e110685.

7. Embley, T.M. (2006). Multiple secondary origins of the anaerobic lifestyle in eukaryotes. Philosophical transactions of the Royal Society of London Series B, Biological sciences 361 , 1055-1067.

8. Koonin, E.V. (2010). The origin and early evolution of eukaryotes in the light of phylogenomics. Genome biology 11, 209.

9. $\quad$ Embley, T.M., and Hirt, R.P. (1998). Early branching eukaryotes? Current opinion in genetics \& development 8, 624-629.

10. Keeling, P.J., Burger, G., Durnford, D.G., Lang, B.F., Lee, R.W., Pearlman, R.E., Roger, A.J., and Gray, M.W. (2005). The tree of eukaryotes. Trends in ecology \& evolution 20, 670-676.

11. Martin, W., and Muller, M. (1998). The hydrogen hypothesis for the first eukaryote. Nature 392, 37-41.

12. Hampl, V., Silberman, J.D., Stechmann, A., Diaz-Trivino, S., Johnson, P.J., and Roger, A.J. (2008). Genetic evidence for a mitochondriate ancestry in the 'amitochondriate' flagellate Trimastix pyriformis. PloS one 3, e1383.

13. Ferla, M.P., Thrash, J.C., Giovannoni, S.J., and Patrick, W.M. (2013). New rRNA gene-based phylogenies of the Alphaproteobacteria provide perspective on major groups, mitochondrial ancestry and phylogenetic instability. PloS one 8, e83383.

14. Muller, M., and Martin, W. (1999). The genome of Rickettsia prowazekii and some thoughts on the origin of mitochondria and hydrogenosomes. BioEssays : news and reviews in molecular, cellular and developmental biology 21, 377-381.

15. Kaneko, T., Nakamura, Y., Sato, S., Minamisawa, K., Uchiumi, T., Sasamoto, S., Watanabe, A., Idesawa, K., Iriguchi, M., Kawashima, K., et al. (2002). Complete genomic sequence of nitrogen-fixing symbiotic bacterium Bradyrhizobium japonicum USDA110. DNA research : an international journal for rapid publication of reports on genes and genomes 9, 189-197.

16. Nierman, W.C., Feldblyum, T.V., Laub, M.T., Paulsen, I.T., Nelson, K.E., Eisen, J.A., Heidelberg, J.F., Alley, M.R., Ohta, N., Maddock, J.R., et al. (2001). Complete genome sequence of Caulobacter crescentus. Proceedings of the National Academy of Sciences of the United States of America 98, 4136-4141.

17. Andersson, S.G., Zomorodipour, A., Andersson, J.O., Sicheritz-Ponten, T., Alsmark, U.C., Podowski, R.M., Naslund, A.K., Eriksson, A.S., Winkler, H.H., and Kurland, C.G. (1998). The genome sequence of Rickettsia prowazekii and the origin of mitochondria. Nature 396, 133140.

18. Lang, B.F., Burger, G., O’Kelly, C.J., Cedergren, R., Golding, G.B., Lemieux, C., Sankoff, D., Turmel, M., and Gray, M.W. (1997). An ancestral mitochondrial DNA resembling a eubacterial genome in miniature. Nature 387, 493-497.

19. Burger, G., Forget, L., Zhu, Y., Gray, M.W., and Lang, B.F. (2003). Unique mitochondrial genome architecture in unicellular relatives of animals. Proceedings of the National Academy of Sciences of the United States of America 100, 892-897.

20. Roe, B.A., Ma, D.P., Wilson, R.K., and Wong, J.F. (1985). The complete nucleotide sequence of the Xenopus laevis mitochondrial genome. The Journal of biological chemistry $260,9759-9774$. 
21. Bayona-Bafaluy, M.P., Acin-Perez, R., Mullikin, J.C., Park, J.S., Moreno-Loshuertos, R., Hu, P., Perez-Martos, A., Fernandez-Silva, P., Bai, Y., and Enriquez, J.A. (2003). Revisiting the mouse mitochondrial DNA sequence. Nucleic acids research 31, 5349-5355.

22. Anderson, S., de Bruijn, M.H., Coulson, A.R., Eperon, I.C., Sanger, F., and Young, I.G. (1982). Complete sequence of bovine mitochondrial DNA. Conserved features of the mammalian mitochondrial genome. Journal of molecular biology 156, 683-717.

23. Lin, C.S., Sun, Y.L., Liu, C.Y., Yang, P.C., Chang, L.C., Cheng, I.C., Mao, S.J., and Huang, M.C. (1999). Complete nucleotide sequence of pig (Sus scrofa) mitochondrial genome and dating evolutionary divergence within Artiodactyla. Gene 236, 107-114.

24. Anderson, S., Bankier, A.T., Barrell, B.G., de Bruijn, M.H., Coulson, A.R., Drouin, J., Eperon, I.C., Nierlich, D.P., Roe, B.A., Sanger, F., et al. (1981). Sequence and organization of the human mitochondrial genome. Nature 290, 457-465.

25. Wilhelm, V., Villegas, J., Miquel, A., Engel, E., Bernales, S., Valenzuela, P.D., and Burzio, L.O. (2003). The complete sequence of the mitochondrial genome of the Chinook salmon, Oncorhynchus tshawytscha. Biological research 36, 223-231.

26. Broughton, R.E., Milam, J.E., and Roe, B.A. (2001). The complete sequence of the zebrafish (Danio rerio) mitochondrial genome and evolutionary patterns in vertebrate mitochondrial DNA. Genome research 11, 1958-1967.

27. Turmel, M., Otis, C., and Lemieux, C. (2002). The complete mitochondrial DNA sequence of Mesostigma viride identifies this green alga as the earliest green plant divergence and predicts a highly compact mitochondrial genome in the ancestor of all green plants. Molecular biology and evolution 19, 24-38.

28. Unseld, M., Marienfeld, J.R., Brandt, P., and Brennicke, A. (1997). The mitochondrial genome of Arabidopsis thaliana contains 57 genes in 366,924 nucleotides. Nature genetics 15, 57-61.

29. Oda, K., Yamato, K., Ohta, E., Nakamura, Y., Takemura, M., Nozato, N., Akashi, K., Kanegae, T., Ogura, Y., Kohchi, T., et al. (1992). Gene organization deduced from the complete sequence of liverwort Marchantia polymorpha mitochondrial DNA. A primitive form of plant mitochondrial genome. Journal of molecular biology 223, 1-7.

30. Ward, B.L., Anderson, R.S., and Bendich, A.J. (1981). The mitochondrial genome is large and variable in a family of plants (cucurbitaceae). Cell 25, 793-803.

31. Cummings, D.J., McNally, K.L., Domenico, J.M., and Matsuura, E.T. (1990). The complete DNA sequence of the mitochondrial genome of Podospora anserina. Current genetics 17, 375-402.

32. Specht, C.A., Novotny, C.P., and Ullrich, R.C. (1992). Mitochondrial DNA of Schizophyllum commune: restriction map, genetic map, and mode of inheritance. Current genetics 22, 129 134.

33. Foury, F., Roganti, T., Lecrenier, N., and Purnelle, B. (1998). The complete sequence of the mitochondrial genome of Saccharomyces cerevisiae. FEBS letters 440, 325-331.

34. Pramateftaki, P.V., Kouvelis, V.N., Lanaridis, P., and Typas, M.A. (2006). The mitochondrial genome of the wine yeast Hanseniaspora uvarum: a unique genome organization among yeast/fungal counterparts. FEMS yeast research 6, 77-90.

35. Anderson, J.B., Wickens, C., Khan, M., Cowen, L.E., Federspiel, N., Jones, T., and Kohn, L.M. (2001). Infrequent genetic exchange and recombination in the mitochondrial genome of Candida albicans. Journal of bacteriology 183, 865-872.

36. Huynen, M.A., Duarte, I., and Szklarczyk, R. (2013). Loss, replacement and gain of proteins at the origin of the mitochondria. Biochimica et biophysica acta 1827, 224-231.

37. Yokobori, S., Suzuki, T., and Watanabe, K. (2001). Genetic code variations in mitochondria: tRNA as a major determinant of genetic code plasticity. Journal of molecular evolution 53, 314326.

38. de Grey, A.D. (2005). Forces maintaining organellar genomes: is any as strong as genetic code disparity or hydrophobicity? BioEssays : news and reviews in molecular, cellular and developmental biology 27, 436-446.

39. Leblanc, C., Richard, O., Kloareg, B., Viehmann, S., Zetsche, K., and Boyen, C. (1997). Origin and evolution of mitochondria: what have we learnt from red algae? Current genetics 31, 193207.

40. Koonin, E.V., Mushegian, A.R., and Bork, P. (1996). Non-orthologous gene displacement. 
Trends in genetics : TIG 12, 334-336.

41. Timmis, J.N., Ayliffe, M.A., Huang, C.Y., and Martin, W. (2004). Endosymbiotic gene transfer: organelle genomes forge eukaryotic chromosomes. Nature reviews Genetics 5, 123-135.

42. Amiri, H., Karlberg, O., and Andersson, S.G. (2003). Deep origin of plastid/parasite ATP/ADP translocases. Journal of molecular evolution 56, 137-150.

43. Boore, J.L. (1999). Animal mitochondrial genomes. Nucleic acids research 27, 1767-1780.

44. Barr, C.M., Neiman, M., and Taylor, D.R. (2005). Inheritance and recombination of mitochondrial genomes in plants, fungi and animals. The New phytologist 168, 39-50.

45. Smeets, H.J. (2013). Preventing the transmission of mitochondrial DNA disorders: selecting the good guys or kicking out the bad guys. Reproductive biomedicine online 27, 599-610.

46. Cummins, J. (1998). Mitochondrial DNA in mammalian reproduction. Reviews of reproduction 3, 172-182.

47. Sato, M., and Sato, K. (2013). Maternal inheritance of mitochondrial DNA by diverse mechanisms to eliminate paternal mitochondrial DNA. Biochimica et biophysica acta 1833, 1979-1984.

48. Song, W.H., Ballard, J.W., Yi, Y.J., and Sutovsky, P. (2014). Regulation of mitochondrial genome inheritance by autophagy and ubiquitin-proteasome system: implications for health, fitness, and fertility. BioMed research international 2014, 981867.

49. Filee, J., Forterre, P., Sen-Lin, T., and Laurent, J. (2002). Evolution of DNA polymerase families: evidences for multiple gene exchange between cellular and viral proteins. Journal of molecular evolution 54, 763-773.

50. Turmel, M., Otis, C., and Lemieux, C. (2003). The mitochondrial genome of Chara vulgaris: insights into the mitochondrial DNA architecture of the last common ancestor of green algae and land plants. The Plant cell 15, 1888-1903.

51. Bullerwell, C.E., and Gray, M.W. (2004). Evolution of the mitochondrial genome: protist connections to animals, fungi and plants. Current opinion in microbiology 7, 528-534.

52. Preuten, T., Cincu, E., Fuchs, J., Zoschke, R., Liere, K., and Borner, T. (2010). Fewer genes than organelles: extremely low and variable gene copy numbers in mitochondria of somatic plant cells. The Plant journal : for cell and molecular biology 64, 948-959.

53. Atkin, O.K., and Macherel, D. (2009). The crucial role of plant mitochondria in orchestrating drought tolerance. Annals of botany 103, 581-597.

54. Van Aken, O., Giraud, E., Clifton, R., and Whelan, J. (2009). Alternative oxidase: a target and regulator of stress responses. Physiologia plantarum 137, 354-361.

55. Jacoby, R.P., Li, L., Huang, S., Pong Lee, C., Millar, A.H., and Taylor, N.L. (2012). Mitochondrial composition, function and stress response in plants. Journal of integrative plant biology 54 , 887-906.

56. Moller, I.M. (2001). PLANT MITOCHONDRIA AND OXIDATIVE STRESS: Electron Transport, NADPH Turnover, and Metabolism of Reactive Oxygen Species. Annual review of plant physiology and plant molecular biology 52, 561-591.

57. Abdelnoor, R.V., Christensen, A.C., Mohammed, S., Munoz-Castillo, B., Moriyama, H., and Mackenzie, S.A. (2006). Mitochondrial genome dynamics in plants and animals: convergent gene fusions of a MutS homologue. Journal of molecular evolution 63, 165-173.

58. Lang, B.F., Laforest, M.J., and Burger, G. (2007). Mitochondrial introns: a critical view. Trends in genetics : TIG 23, 119-125.

59. Aguileta, G., de Vienne, D.M., Ross, O.N., Hood, M.E., Giraud, T., Petit, E., and Gabaldon, T. (2014). High variability of mitochondrial gene order among fungi. Genome biology and evolution 6, 451-465.

60. Himmelstrand, K., Olson, A., Brandstrom Durling, M., Karlsson, M., and Stenlid, J. (2014). Intronic and plasmid-derived regions contribute to the large mitochondrial genome sizes of Agaricomycetes. Current genetics.

61. Birky, C.W., Jr. (1995). Uniparental inheritance of mitochondrial and chloroplast genes: mechanisms and evolution. Proceedings of the National Academy of Sciences of the United States of America 92, 11331-11338.

62. Yang, X., and Griffiths, A.J. (1993). Male transmission of linear plasmids and mitochondrial DNA in the fungus Neurospora. Genetics 134, 1055-1062. 
63. Allen, J.M., Light, J.E., Perotti, M.A., Braig, H.R., and Reed, D.L. (2009). Mutational meltdown in primary endosymbionts: selection limits Muller's ratchet. PloS one 4, e4969.

64. Goyal, S., Balick, D.J., Jerison, E.R., Neher, R.A., Shraiman, B.I., and Desai, M.M. (2012). Dynamic mutation-selection balance as an evolutionary attractor. Genetics 191, 1309-1319.

65. Muller, H.J. (1964). The Relation of Recombination to Mutational Advance. Mutation research 106, 2-9.

66. Lynch, M. (1996). Mutation accumulation in transfer RNAs: molecular evidence for Muller's ratchet in mitochondrial genomes. Molecular biology and evolution 13, 209-220.

67. Lynch, M., Koskella, B., and Schaack, S. (2006). Mutation pressure and the evolution of organelle genomic architecture. Science 311, 1727-1730.

68. Rand, D.M. (2008). Mitigating mutational meltdown in mammalian mitochondria. PLoS biology 6, e35.

69. Gessler, D.D. (1995). The constraints of finite size in asexual populations and the rate of the ratchet. Genetical research 66, 241-253.

70. Howe, D.K., and Denver, D.R. (2008). Muller's Ratchet and compensatory mutation in Caenorhabditis briggsae mitochondrial genome evolution. BMC evolutionary biology 8, 62 .

71. Kaiser, V.B., and Charlesworth, B. (2010). Muller's ratchet and the degeneration of the Drosophila miranda neo-Y chromosome. Genetics 185, 339-348.

72. Bachtrog, D. (2008). The temporal dynamics of processes underlying $Y$ chromosome degeneration. Genetics 179, 1513-1525.

73. Gray, M.W. (2012). Mitochondrial evolution. Cold Spring Harbor perspectives in biology 4, a011403.

74. Brand, M.D. (2010). The sites and topology of mitochondrial superoxide production. Experimental gerontology 45, 466-472.

75. Speijer, D. (2014). How the mitochondrion was shaped by radical differences in substrates: what carnitine shuttles and uncoupling tell us about mitochondrial evolution in response to ROS. BioEssays : news and reviews in molecular, cellular and developmental biology 36, 634643.

76. Gara, L., Pinco, M., and Tomassi, F. (2003). The antioxidant systems vis-à-vis reactive oxygen species during plant-pathogen interaction. Plant physiol Biochem.

77. Boncompain, G., Schneider, B., Delevoye, C., Kellermann, O., Dautry-Varsat, A., and Subtil, A. (2010). Production of reactive oxygen species is turned on and rapidly shut down in epithelial cells infected with Chlamydia trachomatis. Infection and immunity 78, 80-87.

78. Hagstrom, E., Freyer, C., Battersby, B.J., Stewart, J.B., and Larsson, N.G. (2014). No recombination of mtDNA after heteroplasmy for 50 generations in the mouse maternal germline. Nucleic acids research 42, 1111-1116.

79. Elson, J.L., Andrews, R.M., Chinnery, P.F., Lightowlers, R.N., Turnbull, D.M., and Howell, N. (2001). Analysis of European mtDNAs for recombination. American journal of human genetics 68, 145-153.

80. Lynch, M. (2006). The origins of eukaryotic gene structure. Molecular biology and evolution 23, 450-468.

81. Loewe, L. (2006). Quantifying the genomic decay paradox due to Muller's ratchet in human mitochondrial DNA. Genetical research 87, 133-159.

82. Wallace, D.C. (2007). Why do we still have a maternally inherited mitochondrial DNA? Insights from evolutionary medicine. Annual review of biochemistry 76, 781-821.

83. Blanchard, J.L., and Lynch, M. (2000). Organellar genes: why do they end up in the nucleus? Trends in genetics : TIG 16, 315-320.

84. Gawryluk, R.M., and Gray, M.W. (2010). An ancient fission of mitochondrial Cox1. Molecular biology and evolution 27, 7-10.

85. Berg, O.G., and Kurland, C.G. (2000). Why mitochondrial genes are most often found in nuclei. Molecular biology and evolution 17, 951-961.

86. Selosse, M., Albert, B., and Godelle, B. (2001). Reducing the genome size of organelles favours gene transfer to the nucleus. Trends in ecology \& evolution 16, 135-141.

87. Kurihara, Y., Kanki, T., Aoki, Y., Hirota, Y., Saigusa, T., Uchiumi, T., and Kang, D. (2012). 
Mitophagy plays an essential role in reducing mitochondrial production of reactive oxygen species and mutation of mitochondrial DNA by maintaining mitochondrial quantity and quality in yeast. The Journal of biological chemistry 287, 3265-3272.

88. Speijer, D., Manjeri, G.R., and Szklarczyk, R. (2014). How to deal with oxygen radicals stemming from mitochondrial fatty acid oxidation. Philosophical transactions of the Royal Society of London Series B, Biological sciences 369, 20130446.

89. Wimsatt, W.A. (1975). Some comparative aspects of implantation. Biology of reproduction 12, $1-40$.

90. Lee, K.Y., and DeMayo, F.J. (2004). Animal models of implantation. Reproduction 128, 679695.

91. Leese, H.J. (2002). Quiet please, do not disturb: a hypothesis of embryo metabolism and viability. BioEssays : news and reviews in molecular, cellular and developmental biology 24 , 845-849.

92. May-Panloup, P., Vignon, X., Chretien, M.F., Heyman, Y., Tamassia, M., Malthiery, Y., and Reynier, P. (2005). Increase of mitochondrial DNA content and transcripts in early bovine embryogenesis associated with upregulation of mtTFA and NRF1 transcription factors. Reproductive biology and endocrinology : RB\&E 3, 65.

93. Thundathil, J., Filion, F., and Smith, L.C. (2005). Molecular control of mitochondrial function in preimplantation mouse embryos. Molecular reproduction and development 71, 405-413.

94. Wolff, J.N., and Gemmell, N.J. (2008). Lost in the zygote: the dilution of paternal mtDNA upon fertilization. Heredity 101, 429-434.

95. Davila, J.I., Arrieta-Montiel, M.P., Wamboldt, Y., Cao, J., Hagmann, J., Shedge, V., Xu, Y.Z., Weigel, D., and Mackenzie, S.A. (2011). Double-strand break repair processes drive evolution of the mitochondrial genome in Arabidopsis. BMC biology 9, 64 .

96. Galtier, N. (2011). The intriguing evolutionary dynamics of plant mitochondrial DNA. BMC biology $9,61$.

97. Sia, E.A., and Kirkpatrick, D.T. (2005). The yeast MSH1 gene is not involved in DNA repair or recombination during meiosis. DNA repair 4, 253-261.

98. Ricchetti, M., Fairhead, C., and Dujon, B. (1999). Mitochondrial DNA repairs double-strand breaks in yeast chromosomes. Nature 402, 96-100.

99. Christensen, A.C. (2013). Plant mitochondrial genome evolution can be explained by DNA repair mechanisms. Genome biology and evolution 5, 1079-1086.

100. Marechal, A., and Brisson, N. (2010). Recombination and the maintenance of plant organelle genome stability. The New phytologist 186, 299-317.

101. Cao, L., Shitara, H., Horii, T., Nagao, Y., Imai, H., Abe, K., Hara, T., Hayashi, J., and Yonekawa, $\mathrm{H}$. (2007). The mitochondrial bottleneck occurs without reduction of mtDNA content in female mouse germ cells. Nature genetics 39, 386-390.

102. Cree, L.M., Samuels, D.C., de Sousa Lopes, S.C., Rajasimha, H.K., Wonnapinij, P., Mann, J.R., Dahl, H.H., and Chinnery, P.F. (2008). A reduction of mitochondrial DNA molecules during embryogenesis explains the rapid segregation of genotypes. Nature genetics 40, 249-254.

103. Bergstrom, C.T., and Pritchard, J. (1998). Germline bottlenecks and the evolutionary maintenance of mitochondrial genomes. Genetics 149, 2135-2146.

104. Ashley, M.V., Laipis, P.J., and Hauswirth, W.W. (1989). Rapid segregation of heteroplasmic bovine mitochondria. Nucleic acids research 17, 7325-7331.

105. Piko, L., and Taylor, K.D. (1987). Amounts of mitochondrial DNA and abundance of some mitochondrial gene transcripts in early mouse embryos. Developmental biology 123, 364-374.

106. Wolff, J.N., White, D.J., Woodhams, M., White, H.E., and Gemmell, N.J. (2011). The strength and timing of the mitochondrial bottleneck in salmon suggests a conserved mechanism in vertebrates. PloS one 6, e20522.

107. Wai, T., Teoli, D., and Shoubridge, E.A. (2008). The mitochondrial DNA genetic bottleneck results from replication of a subpopulation of genomes. Nature genetics 40, 1484-1488.

108. Guo, Y., Li, C.I., Sheng, Q., Winther, J.F., Cai, Q., Boice, J.D., and Shyr, Y. (2013). Very low-level heteroplasmy mtDNA variations are inherited in humans. Journal of genetics and genomics $=Y i$ chuan xue bao 40, 607-615. 
109. Stewart, J.B., Freyer, C., Elson, J.L., Wredenberg, A., Cansu, Z., Trifunovic, A., and Larsson, N.G. (2008). Strong purifying selection in transmission of mammalian mitochondrial DNA. PLoS biology 6, e10.

110. Bazin, E., Glemin, S., and Galtier, N. (2006). Population size does not influence mitochondrial genetic diversity in animals. Science $312,570-572$.

111. Meiklejohn, C.D., Montooth, K.L., and Rand, D.M. (2007). Positive and negative selection on the mitochondrial genome. Trends in genetics : TIG 23, 259-263.

112. Fan, W., Waymire, K.G., Narula, N., Li, P., Rocher, C., Coskun, P.E., Vannan, M.A., Narula, J., Macgregor, G.R., and Wallace, D.C. (2008). A mouse model of mitochondrial disease reveals germline selection against severe mtDNA mutations. Science 319, 958-962.

113. Shoubridge, E.A., and Wai, T. (2008). Medicine. Sidestepping mutational meltdown. Science 319, 914-915.

114. Ebert, K.M., Liem, H., and Hecht, N.B. (1988). Mitochondrial DNA in the mouse preimplantation embryo. Journal of reproduction and fertility 82, 145-149.

115. Wai, T., Ao, A., Zhang, X., Cyr, D., Dufort, D., and Shoubridge, E.A. (2010). The role of mitochondrial DNA copy number in mammalian fertility. Biology of reproduction 83, 52-62.

116. Ma, H., Xu, H., and O'Farrell, P.H. (2014). Transmission of mitochondrial mutations and action of purifying selection in Drosophila melanogaster. Nature genetics 46, 393-397.

117. Hill, J.H., Chen, Z., and Xu, H. (2014). Selective propagation of functional mitochondrial DNA during oogenesis restricts the transmission of a deleterious mitochondrial variant. Nature genetics 46, 389-392.

118. Twig, G., Elorza, A., Molina, A.J., Mohamed, H., Wikstrom, J.D., Walzer, G., Stiles, L., Haigh, S.E., Katz, S., Las, G., et al. (2008). Fission and selective fusion govern mitochondrial segregation and elimination by autophagy. The EMBO journal 27, 433-446.

119. Glauser, L., Sonnay, S., Stafa, K., and Moore, D.J. (2011). Parkin promotes the ubiquitination and degradation of the mitochondrial fusion factor mitofusin 1. Journal of neurochemistry 118 , 636-645

120. Bastiaans, E., Aanen, D.K., Debets, A.J., Hoekstra, R.F., Lestrade, B., and Maas, M.F. (2014). Regular bottlenecks and restrictions to somatic fusion prevent the accumulation of mitochondrial defects in Neurospora. Philosophical transactions of the Royal Society of London Series B, Biological sciences 369, 20130448.

121. Duran, H.E., Simsek-Duran, F., Oehninger, S.C., Jones, H.W., Jr., and Castora, F.J. (2011). The association of reproductive senescence with mitochondrial quantity, function, and DNA integrity in human oocytes at different stages of maturation. Fertility and sterility 96, 384-388.

122. Santos, T.A., El Shourbagy, S., and St John, J.C. (2006). Mitochondrial content reflects oocyte variability and fertilization outcome. Fertility and sterility 85, 584-591.

123. Reynier, P., May-Panloup, P., Chretien, M.F., Morgan, C.J., Jean, M., Savagner, F., Barriere, P., and Malthiery, Y. (2001). Mitochondrial DNA content affects the fertilizability of human oocytes. Molecular human reproduction 7, 425-429.

124. May-Panloup, P., Chretien, M.F., Jacques, C., Vasseur, C., Malthiery, Y., and Reynier, P. (2005). Low oocyte mitochondrial DNA content in ovarian insufficiency. Human reproduction 20, 593597.

125. Monnot, S., Samuels, D.C., Hesters, L., Frydman, N., Gigarel, N., Burlet, P., Kerbrat, V., Lamazou, F., Frydman, R., Benachi, A., et al. (2013). Mutation dependance of the mitochondrial DNA copy number in the first stages of human embryogenesis. Human molecular genetics 22 , 1867-1872.

126. Chan, C.C., Liu, V.W., Lau, E.Y., Yeung, W.S., Ng, E.H., and Ho, P.C. (2005). Mitochondrial DNA content and 4977 bp deletion in unfertilized oocytes. Molecular human reproduction 11, 843-846.

127. Murakoshi, Y., Sueoka, K., Takahashi, K., Sato, S., Sakurai, T., Tajima, H., and Yoshimura, Y. (2013). Embryo developmental capability and pregnancy outcome are related to the mitochondrial DNA copy number and ooplasmic volume. Journal of assisted reproduction and genetics 30, 1367-1375.

128. Ge, H., Tollner, T.L., Hu, Z., Dai, M., Li, X., Guan, H., Shan, D., Zhang, X., Lv, J., Huang, C., et al. (2012). The importance of mitochondrial metabolic activity and mitochondrial DNA replication 
during oocyte maturation in vitro on oocyte quality and subsequent embryo developmental competence. Molecular reproduction and development 79, 392-401.

129. Wang, Q., Ratchford, A.M., Chi, M.M., Schoeller, E., Frolova, A., Schedl, T., and Moley, K.H. (2009). Maternal diabetes causes mitochondrial dysfunction and meiotic defects in murine oocytes. Molecular endocrinology 23, 1603-1612.

130. Ou, X.H., Li, S., Wang, Z.B., Li, M., Quan, S., Xing, F., Guo, L., Chao, S.B., Chen, Z., Liang, X.W., et al. (2012). Maternal insulin resistance causes oxidative stress and mitochondrial dysfunction in mouse oocytes. Human reproduction 27, 2130-2145.

131. Zhang, W., Liu, Y., An, Z., Huang, D., Qi, Y., and Zhang, Y. (2011). Mediating effect of ROS on mtDNA damage and low ATP content induced by arsenic trioxide in mouse oocytes. Toxicology in vitro : an international journal published in association with BIBRA 25, 979-984.

132. Zeng, H.T., Yeung, W.S., Cheung, M.P., Ho, P.C., Lee, C.K., Zhuang, G.L., Liang, X.Y., and O, W.S. (2009). In vitro-matured rat oocytes have low mitochondrial deoxyribonucleic acid and adenosine triphosphate contents and have abnormal mitochondrial redistribution. Fertility and sterility 91, 900-907.

133. Kameyama, Y., Filion, F., Yoo, J.G., and Smith, L.C. (2007). Characterization of mitochondrial replication and transcription control during rat early development in vivo and in vitro. Reproduction 133, 423-432.

134. El Shourbagy, S.H., Spikings, E.C., Freitas, M., and St John, J.C. (2006). Mitochondria directly influence fertilisation outcome in the pig. Reproduction 131, 233-245.

135. Gil, M.A., Maside, C., Cuello, C., Parrilla, I., Vazquez, J.M., Roca, J., and Martinez, E.A. (2012). Effects of Hoechst 33342 staining and ultraviolet irradiation on mitochondrial distribution and DNA copy number in porcine oocytes and preimplantation embryos. Molecular reproduction and development 79, 651-663.

136. Mao, J., Whitworth, K.M., Spate, L.D., Walters, E.M., Zhao, J., and Prather, R.S. (2012). Regulation of oocyte mitochondrial DNA copy number by follicular fluid, EGF, and neuregulin 1 during in vitro maturation affects embryo development in pigs. Theriogenology 78, 887-897.

137. Pawlak, P., Renska, N., Pers-Kamczyc, E., Warzych, E., and Lechniak, D. (2011). The quality of porcine oocytes is affected by sexual maturity of the donor gilt. Reproductive biology 11, 1-18.

138. Sato, D., Itami, N., Tasaki, H., Takeo, S., Kuwayama, T., and Iwata, H. (2014). Relationship between mitochondrial DNA copy number and SIRT1 expression in porcine oocytes. PloS one 9, e94488.

139. Spikings, E.C., Alderson, J., and St John, J.C. (2007). Regulated mitochondrial DNA replication during oocyte maturation is essential for successful porcine embryonic development. Biology of reproduction 76, 327-335.

140. Santos, E.C., Sato, D., Lucia, T., and Iwata, H. (2013). Brilliant cresyl blue staining negatively affects mitochondrial functions in porcine oocytes. Zygote, 1-8.

141. Michaels, G.S., Hauswirth, W.W., and Laipis, P.J. (1982). Mitochondrial DNA copy number in bovine oocytes and somatic cells. Developmental biology 94, 246-251.

142. Hua, S., Zhang, Y., Li, X.C., Ma, L.B., Cao, J.W., Dai, J.P., and Li, R. (2007). Effects of granulosa cell mitochondria transfer on the early development of bovine embryos in vitro. Cloning and stem cells 9, 237-246.

143. Takeo, S., Sato, D., Kimura, K., Monji, Y., Kuwayama, T., Kawahara-Miki, R., and Iwata, H. (2014). Resveratrol improves the mitochondrial function and fertilization outcome of bovine oocytes. The Journal of reproduction and development 60, 92-99.

144. Iwata, H., Goto, H., Tanaka, H., Sakaguchi, Y., Kimura, K., Kuwayama, T., and Monji, Y. (2011). Effect of maternal age on mitochondrial DNA copy number, ATP content and IVF outcome of bovine oocytes. Reproduction, fertility, and development 23, 424-432.

145. Cotterill, M., Harris, S.E., Collado Fernandez, E., Lu, J., Huntriss, J.D., Campbell, B.K., and Picton, H.M. (2013). The activity and copy number of mitochondrial DNA in ovine oocytes throughout oogenesis in vivo and during oocyte maturation in vitro. Molecular human reproduction 19, 444-450.

146. Zampolla, T., Spikings, E., Rawson, D., and Zhang, T. (2011). Cytoskeleton proteins F-actin and tubulin distribution and interaction with mitochondria in the granulosa cells surrounding stage III zebrafish (Danio rerio) oocytes. Theriogenology 76, 1110-1119. 



\section{Chapter 3}

\section{Differences in strength and timing of the}

\section{mtDNA bottleneck between zebrafish}

\section{germline and non-germline cells}

Auke BC Otten, Tom EJ Theunissen, Josien G Derhaag, Ellen H Lambrichs, Iris BW Boesten, Marie Winandy, Aafke PA van Montfoort, Katsiaryna

Tarbashevich, Erez Raz, Mike Gerards, Jo M Vanoevelen, Bianca JC van den Bosch, Marc Muller, Hubert JM Smeets 


\section{Summary}

We studied the mtDNA bottleneck in zebrafish to elucidate size, timing and variation in germline and non-germline cells. Mature zebrafish oocytes contain, on average, $19.0 \times 10^{6} \mathrm{mtDNA}$ molecules with high variation between oocytes. During embryogenesis, the mtDNA copy number decreases to 170 mtDNA molecules per primordial germ cell (PGC), a number similar to mammals, and to $\sim 50$ per non-PGC. These occur at the same developmental stage, implying considerable variation in mtDNA copy number in (non-)PGCs of the same female, dictated by variation in the mature oocyte. The presence of oocytes with low mtDNA numbers, if similar in humans, could explain that (de novo) mutations can reach high mutation loads within a single generation. High mtDNA copy numbers in mature oocytes are established by mtDNA replication during oocyte development. Bottleneck differences between germline and non-germline cells, due to early differentiation of PGCs, may account for different distribution patterns of familial mutations.

\section{Introduction}

Various mechanisms have evolved to manage the high mitochondrial DNA (mtDNA) mutation rate ${ }^{1}$. In animals, a high mtDNA copy number in cells dilutes the effect of mtDNA mutations ${ }^{2}$. Heteroplasmic mutations can only manifest above a tissueand mutation-specific threshold. Another mechanism is the mtDNA bottleneck during maternal inheritance: a limited amount of mtDNA of the oocytes repopulates the cells of the next generation, thereby filtering out low-level mtDNA mutations. As a result, individuals are usually homoplasmic ${ }^{3}$, which is the healthiest situation ${ }^{4}$. However, in case of familial pathogenic mutations, the bottleneck can cause high and unpredictable shifts in the mtDNA mutation load and disease risks in the offspring ${ }^{5}$.

Despite extensive research, the mtDNA bottleneck is still not fully understood. Data from Holstein cows ${ }^{6}$ indicated that the bottleneck was caused by a sharp decrease in mtDNA copy number during early development, most likely, followed by a clonal expansion of these founder molecules during oogenesis. In mice, a similar decrease in the mtDNA copy number between oocytes (range of $\left.10^{5}\right)^{7 ; 8}$ and primordial germ cells (PGCs) was reported with $\sim 200$ mtDNA molecules in a single PGC at the bottom of the bottleneck, in line with previous estimates $(185)^{9}$. In contrast, in another study, 2000 mtDNA molecules were measured in a single PGC ${ }^{10}$ and a small effective number of segregational units was proposed to explain the rapid segregation, either by assembly of mtDNA molecules into nucleoids or due to preferential replication of a subpopulation of the mtDNA genome ${ }^{8 ; 10}$. For salmons, it was reported that the bottleneck occurred during oogenesis with a size of about 85 mtDNA copies ${ }^{11}$. In humans, indirect estimations of the bottleneck size have been described, ranging from only $1-5^{12}$ and $30-35^{13}$ to $\sim 90^{14}$ and $180^{5}$ copies.

We measured mtDNA copy number and variation in zebrafish oocytes and in larval germline and non-germline cells during embryonic development. The zebrafish model allowed relatively easy collection of mature oocytes from individual female fish, allowing assessment of the individual variation. Furthermore, PGCs were specifically visualized with GFP followed by fluorescence activated cell sorting 
(FACS) to isolate both PGCs and non-PGCs during embryogenesis ${ }^{15}$. Lastly, oocytes from different stages of development were isolated. In this way, we characterized the mtDNA bottleneck in zebrafish in germline and non-germline cells, providing a better understanding of the underlying mechanism and possible consequences.

\section{Material and Methods}

\section{Zebrafish embryos and oocytes}

$A B$ zebrafish embryos were raised, housed and staged according to standard procedures ${ }^{16}$. Mature and immature oocytes were collected manually as described before $^{17}$.

\section{Isolation of primordial germ cells}

Primordial germ cells were identified by both mRNA injections and by a transgenic line. Artificial mRNA constructs with the GFP open reading frame fused to the 3'UTR of zebrafish nanos 3 was prepared as described before ${ }^{15}$. For the transgenic line, the kop-EGFP-F'-nos3-'UTR-cry-DsRed transgene cassette was cloned into the pTolDest vector. Injected or transgenic animals were selected per 100 on the basis of showing similar GFP intensity and no ectopic GFP-expression. Embryos were completely disaggregated and cells were filtered. Subsequently, FACS-sorting (BD Biosciences FACSAria II) was performed as described before ${ }^{15}$.

\section{Quantification of mtDNA copy number}

After DNA extraction, the mtDNA copy number was measured. Absolute mtDNA copy number quantification was performed by real-time quantitative PCR (Q-PCR) of the zebrafish mt-nd1 (NADH dehydrogenase 1, mitochondrial) gene. Amplification results were converted to absolute mtDNA copy number using a standard curve. Relative quantification of the mtDNA copy number was performed by Q-PCR measurements of the steady-state amounts of both $m$ tnd 1 and $b 2 m$ (nuclear gene beta-2-microglobulin). For the 256-cell stage, we measured the mtDNA copy number both absolutely and relatively, allowing an estimation of the absolute mtDNA copy numbers in embryos staged 512-cell or older (Table S3.1).

\section{Next Generation sequencing}

Next Generation Sequencing (NGS) was performed on an Illumina HiSeq 2000. Heteroplasmy values were calculated as the ration of the nucleotides over the total count of any nucleotide at a position.

\section{Statistical analysis}

Statistical analyses were carried out using GraphPad Prism Version 5.02 software. Gaussian distribution was tested using the d'Agostino \& Pearson omnibus normality test. One-way analysis of variance (ANOVA) and the Bonferroni multiple comparison 
test were used for comparing multiple groups. Spearman's rank correlation coefficient was used for analyzing trends. Non-linear regression analysis was performed using the one-phase decay exponential equation. Calculated $P$-values were considered significant $<0.05$. Coefficients of variation (CVs) were calculated as the ratio between the standard deviation and the mean.

\section{Results}

\section{mtDNA copy number and variation in mature zebrafish oocytes}

The average number of mtDNA molecules in 186 individual mature oocytes (Figure 3.1A, S1) was $19.0 \times 10^{6}$ (Range: $3.3 \times 10^{6}-42.2 \times 10^{6}$, Table S3.1), but the distribution was non-Gaussian (Figure 3.1A; $P$-value: 0.014 ). We excluded polymorphisms in the primer binding sites as a cause of these variations by sequencing these sites in eight female zebrafish and 10-15 of their oocytes in the $A B$ strain used (Table S3.2 ${ }^{18}$ ). Among these oocytes, 137 were collected from only eight different females [a single batch with ages at both the start (3-6 months) and end of the fertile period (18-24 months)] and the mean values among these females were statistically similar (all $P$-values $>0.05$, Figure $3.1 \mathrm{~B}$ ), with all mean values $\sim 20 \times 10^{6}$, implying no differences in oocytes derived from females from the same batch, early (fish A-D) and late (fish $\mathrm{E}-\mathrm{H}$ ) in their fertile period. The individual frequency distribution of the mtDNA content in oocytes fits a Gaussian distribution ( $P$-values $>0.05)$. High variation in the mtDNA copy number exists within the oocytes from a single female fish (highest value 3-7 times the lowest value, Table S3.1).

\section{mtDNA copy number during zebrafish embryonic development}

To assess the mtDNA copy number during development we collected embryos and larvae from different stages from one batch of eggs from 5-10 individual females. In the cleavage and early blastula stages, an embryo consists of a fixed number of cells and absolute copy numbers per cell were assessed [1- (mature oocyte) to 256-cell; Figure 3.1C]. The mtDNA copy numbers of the 1-cell stage (mature oocytes) are at the lower bound of the distribution of the mtDNA copy number in all oocytes (Figure 3.1A). As the different isolation methods used gave comparable results (Figure S3.1), this most likely reflects biological differences between the female fish (from different batches). Dividing the mtDNA copy number by the cell number shows that the average mtDNA copy number per cell was halved every cell division, indicating the total number of mtDNA is stable and mtDNA replication is absent.

From the 512-cell stage until the prim-22 stage, we measured the mtDNA copy number relative to a nuclear gene ( $m$ tnd1/b2m, Figure 3.1D-E). The decrease in mtDNA load continued during late blastula -and early gastrula stages (Figure 3.1D), but stopped during segmentation (Figure 1E). Based on the relative and absolute quantification performed on the 256-cell stage, we estimated the amount of mtDNA at this point to be 12-56 mtDNA copies per cell (Table S3.1). 
A

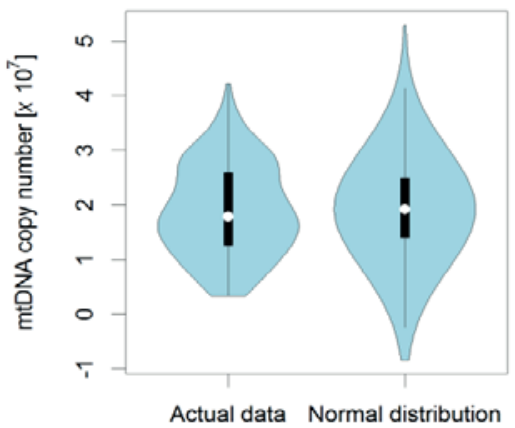

C

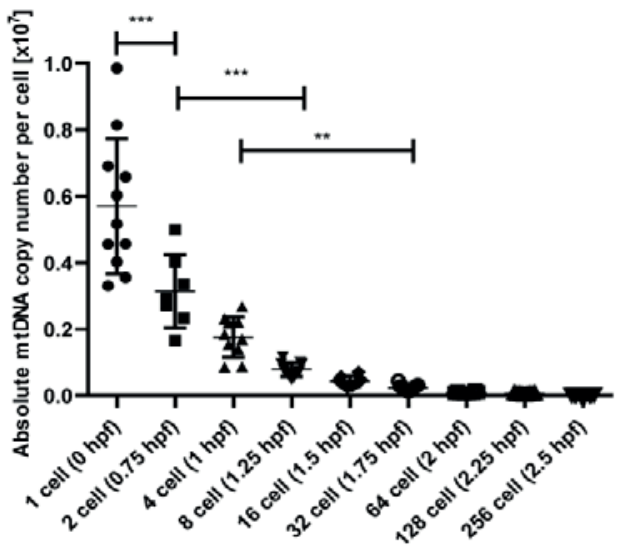

B

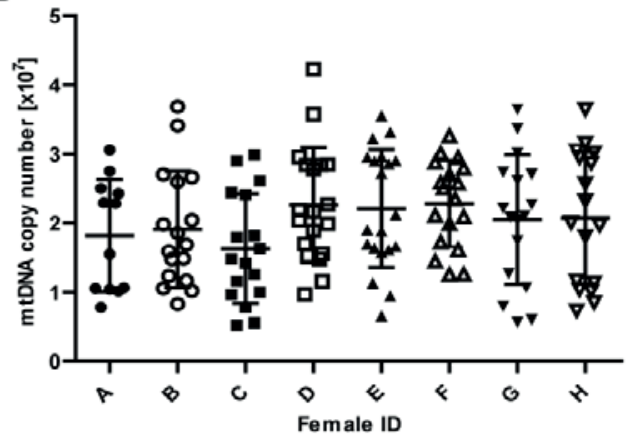

D



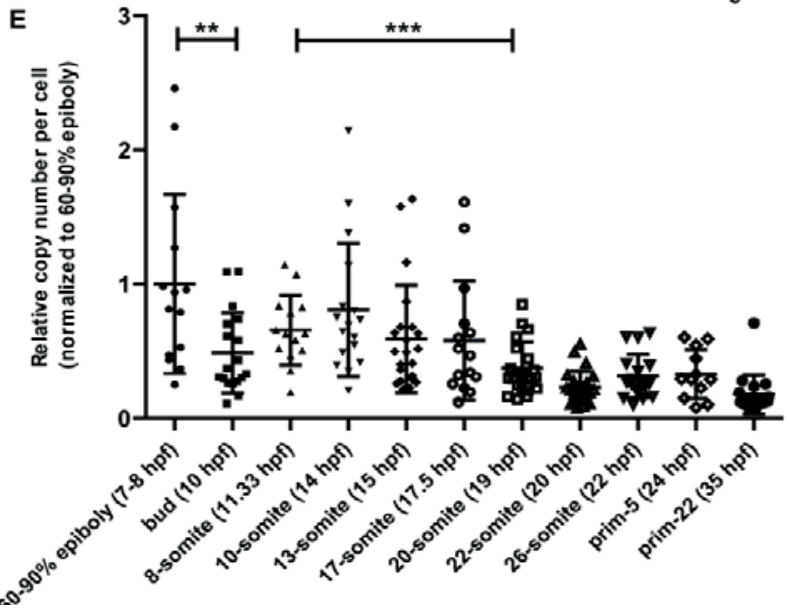

Figure 3.1 | The mtDNA copy number in zebrafish mature oocytes and during embryonic development. (A) Violin plot of absolute mtDNA copy number in 186 oocytes (left) compared with that of a Gaussian distribution (with same SD). White dot corresponds to the median value and the black bar to the $25^{\text {th }}$ (bottom) and $75^{\text {th }}$ (top) percentile value. (B) Absolute oocyte mtDNA copy number in the 137 oocytes from eight females, each symbol represents a single oocyte. Fish A-D were at the start and fish E-H at the end of the fertile period. (C) Absolute quantification of the mtDNA copy number per cell in single embryos staged 1-256-cell. (D-E) Relative quantification ( $m t-n d 1 / \mathrm{b} 2 m$ ) of the mtDNA copy number (per cell) in single embryos staged (D) 512-cell until shield, normalized to 512-cell stage and (E) 60\%-90 epiboly until prim-22, normalized to the 60-90\% epiboly stage. ${ }^{*} P$-values $<0.05$, ${ }^{* *} P$-values $<$ $0.01,{ }^{* * *} P$-values $<0.001$ after one-way ANOVA analyis. Horizontal lines indicate the mean value with SD. 


\section{Isolation of germline cells during zebrafish embryogenesis}

PGCs were visualized by injection of egfp-nanos3 3'UTR mRNA and by generating a transgenic line expressing EGFP-nanos3 3'UTR (Figure 3.2A). Both methods generated similar GFP expression patterns and a specific and representative staining of PGCs, but injected embryos had higher rates of ectopic GFP expression (e.g. in the brain; excluded from further analysis). After enzymatic dissociation, cell suspensions were FACS-analyzed (gating as in ${ }^{15}$ ) and sorted based on their morphology and GFP intensity. After removal of aggregates (enrichment run), cells displaying the highest GFP fluorescence were all located in a narrow range on the FSC channel (purity run), indicating a morphologically homogeneous cell population. Microscopic analysis confirmed that $>95 \%$ of these isolated PGCs presented high GFP intensity (Figure S3.1).

\section{The germline and non-germline mtDNA bottleneck size during zebrafish embryogenesis}

PGCs from embryos of $8,24,48$ and 72 hours post fertilization (hpf) had a significantly lower mtDNA copy number compared to the oocytes (all $P$-values $<0.001$; Figure 3.2B). Although no significant differences were observed from stage 8-72 hpf (all $P$-values $>0.05)$, a correlation analysis revealed a significant downward trend $(P$-value $=0.0009)$ in mtDNA copy number in migratory PGCs following their arrival at the region where the gonad develops (Figure 3.2D ${ }^{19}$ ). The mean values, standard deviation, range and CVs (Table S3.1) indicated that the lowest mtDNA copy number in a single PGC was $170 \mathrm{mtDNA}$ molecules at $72 \mathrm{hpf}$. The variation was high during all stages of development (Table S3.1). Correlation analysis also revealed a downward trend in the mtDNA copy number in non-PGCs during development $(P$-value $=0.0097$, Figure $3.2 \mathrm{C})$. The lowest mtDNA load observed in single nonPGCs ( 50, Table S3.1) was not significantly different from the value measured for the PGCs; however both slopes (PGCs vs non-PGCs) differed statistically from each other $(P$-value $<0.0001)$. The value of $\sim 50$ is similar to the estimated non-germline bottleneck size in whole embryos. Again, variation in bottleneck size is large in nonPGCs from all developmental stages (Table S3.1). Non-linear regression analysis, based on the assumption that the mtDNA load is equally divided among the daughter cells during development, fits the measured mtDNA copy values best $\left(R^{2}=0.75\right.$ and 0.55 respectively). We estimated the plateau value ( $\approx$ bottleneck size) for PGCs at 193.5 (Standard error: 56.3) and for non-PGCs at 89.4 (Standard error: 16.3) mtDNA copies, which is close to the actual lowest mtDNA counts measured in this study. One phase decay equations (Figure 3.2D) indicated that the plateau value is reached earlier in non-PGCs than in PGCs, which is corroborated by the differences in the half times (6.9 hpf for PGCs and $3.4 \mathrm{hpf}$ for non-PGCs). 
A
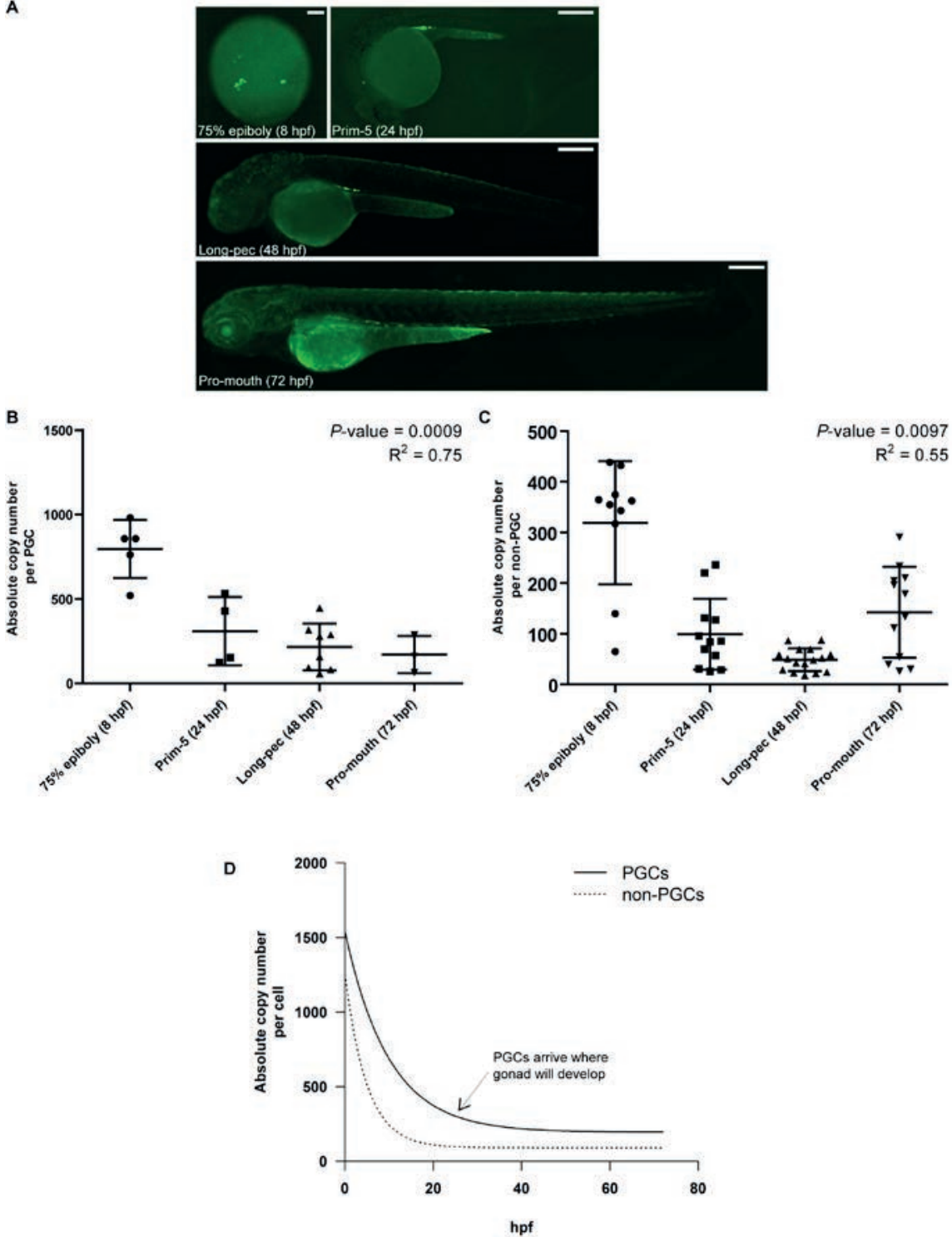

Figure 3.2 The mtDNA copy number in FACS-isolated PGCs and non-PGCs from zebrafish embryos. GFP expression in transgenic zebrafish embryos (EGFP-nanos3 3'UTR). Embryos were imaged using an Axioplan M1 Zeiss microscope. The images represent a combination of pictures captured along the anterior-posterior axis of the embryo, using specific focal planes Scale bars: $100 \mu \mathrm{m}$ for $8 \mathrm{hpf}, 200 \mu \mathrm{m}$ for 24, 48 and 72 hpf. (B-C) Absolute mtDNA copy number in (B) PGCs and (C) nonPGCs from various stages of development. Every symbol represents one group of 80 cells. Horizontal lines indicate mean with SD. $P$-values are generated by Spearman's rank correlation test and indicate a downward trend when $<0.05$. $R^{2}$ indicates fitness of a non-linear one-phase decay exponential equation. (D) Plot of the non-linear one-phase decay equation for PGCs (derived from graph in panel B) and nonPGCs (derived from panel C). The half-life was $6.9 \mathrm{hpf}$ for PGCs and $3.4 \mathrm{hpf}$ for non-PGCs. 


\section{mtDNA copy number during oocyte development}

Stage I oocytes, the primary growth phase, had an average diameter of $115 \mu \mathrm{m}$ and possessed $\sim 840,000$ mtDNA molecules (Figure 3.3A). This number was significantly higher in stage II oocytes, the cortical alveolus stage, ranging from $5.0 \times 10^{6}$ in small stage II oocytes ( $\mathrm{II}_{\text {small }}$; average diameter $191 \mu \mathrm{m}$ ) to $11.1 \times 10^{6}$ in large stage II oocytes (II ${ }_{\text {large }}$; average diameter $256 \mu \mathrm{m}$ ). During stage III, the vitellogenesis stage, the mtDNA copy number per oocyte increased to $14.3 \times 10^{6}$ (average diameter $370 \mu \mathrm{m}$ ). Eventually, stage $V$ (mature) oocytes (average diameter $750 \mu \mathrm{m}$ ) contained $17.4 \times 10^{6}$ mtDNA copies. The variation was higher in stage I oocytes (CV: 106\%), compared to the other stages (CVs: 40-52\%; Table S3.1). We observed a tight positive logarithmic correlation between the volume of the oocytes stage I-III (assuming a sphere) and the mtDNA content (Figure 3.3B).

A

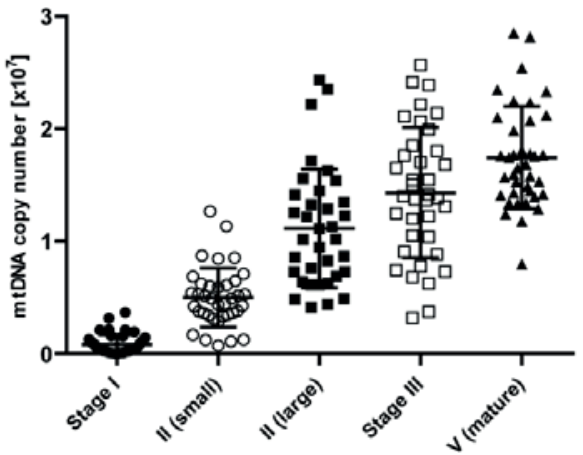

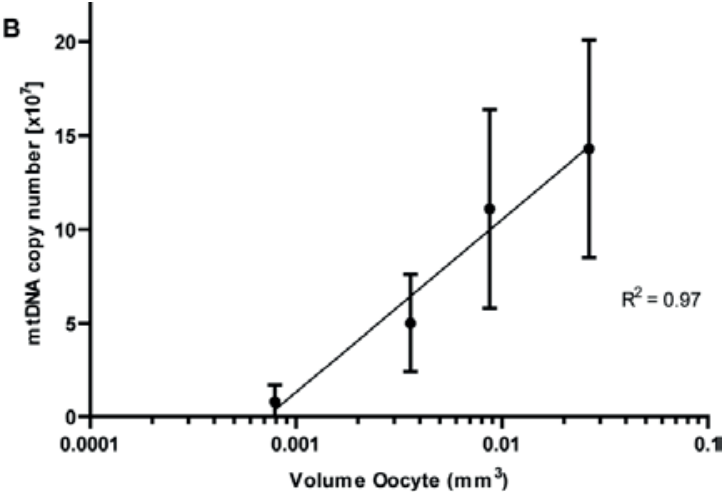

Figure 3.3 | The mtDNA copy number correlates to an increased volume during oocyte maturation. (A) Mature and immature oocytes from different developmental stages were isolated from 4 different females and mtDNA copy number was measured. Each symbol represents a single (immature) oocyte. Horizontal lines indicate mean with SD. All groups were statistically different from each other. (B) Relation between the oocyte volume, assuming a sphere, and the mtDNA amount in oocytes stage I-III.. $\mathrm{R}^{2}$ indicate fitness after a linear regression analysis between the volume (logaritmic) and the mtDNA copy number.

\section{Discussion}

\section{High and variable mtDNA copy number in mature zebrafish oocytes}

The mtDNA load in mature zebrafish oocytes was, on average, $19.0 \times 10^{6} \mathrm{mtDNA}$ copies per cell (Figure 3.1A-B). The distribution of all oocytes indicated a high, nonGaussian variation (range: $3.3 \times 10^{6}-42.2 \times 10^{6}$ ), with a sharp lower-boundary value of $3.3 \times 10^{6}$, while the limitation on high mtDNA loads seems to be more moderate (up to $\left.42.2 \times 10^{6}\right)$. The mean oocyte mtDNA copy number among the eight different female fish was statistically similar (Figure 3.1B), suggesting low inter-individual variation. We observed variation in the DNA copy number among different batches of fish. Batch effects have been reported in zebrafish, for example, in transcriptomics ${ }^{20}$. Our data are comparable with the reported copy number in single zebrafish blastomeres of a 4 -cell stage $\left(14 \times 10^{6}\right.$ copies per cell $\left.{ }^{21}\right)$, corresponding to an mtDNA load of $56 \times 10^{6}$ 
in oocytes, which, although in the same order of magnitude, indicates again biological variation among batches. Oocytes from salmons, another teleost species, contain

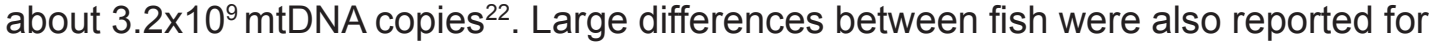
salmon, ranging from $1.1 \times 10^{9}$ to $7.0 \times 10^{9}$ (factor $7^{2}$ ).

In most mammals, mature oocytes contain less than $1.0 \times 10^{6} \mathrm{mtDNA}$ copies, ranging from 110,000 in $R$. Norvegicus ${ }^{23}$ to 807,794 in $B$. Taurus ${ }^{24}$, with substantial variation within species ${ }^{2}$, e.g. in humans ranging from $143,000^{25}$ to $697,176^{26}$. For humans, mice and cows the average reported mtDNA load in the oocytes is below 300,000 copies $^{2}$, suggesting zebrafish contain about hundred times more mtDNA in their oocytes than mammals. This differences is most likely due to differences in implantation and in oxidative activity needed to satisfy the higher energetic demands in teleost compared to mammals due a dependency on fatty acid oxidation ${ }^{22}$. Differences in the mtDNA load of oocytes are also reflected by differences in the size of the oocytes, which are smallest in mammals $(<0.15 \mathrm{~mm}$, human oocytes $0.1 \mathrm{~mm})$, moderate in zebrafish $\left(0.75 \mathrm{~mm}^{16 ; 17}\right)$ and largest in salmons $\left(>4.5 \mathrm{~mm}^{22}\right)$. Based on these volumes, we estimated that the mtDNA copy number per unit of volume is fairly equal across species. This is corroborated by a study in ovine oocytes, where the mtDNA copy number per unit oocyte volume showed only little variation, much lower compared to the variation in the mtDNA copy number in the whole oocyte ${ }^{27}$.

A selection against oocytes with low mtDNA load seems to occur (Figure 3.1A). The non-Gaussian distribution of the mtDNA copy number is most likely due to a sharp boundary at the lowest mtDNA copy number. No oocytes with less than $3.3 \times 10^{6}$ mtDNA copies have been observed. Mitochondrial function and copy number are important for successful fertilization ${ }^{28}$. Low mitochondrial content has been demonstrated to negatively influence the fertilizability of eggs, probably due to inadequate mitochondrial biogenesis or cytoplasmic maturation ${ }^{29}$, which has been demonstrated by low mtDNA counts in degenerate oocytes ${ }^{30}$, oocytes with fertilization failure ${ }^{29}$ or during ovarian insufficiency ${ }^{31}$. This was confirmed by studies in mice ${ }^{32}$. In contrast, an upper mtDNA threshold has been proposed in human blastocysts, above which implantation was never observed and aneuploidy was more frequent ${ }^{33}$.

For each individual female fish, the inter-oocyte variation in mtDNA copy number was high (3 to 8 fold, Figure 3.1B). As we collected unfertilized stage V (mature) oocytes ${ }^{17}$ by squeezing the abdomen of female fish after they were kept with a male overnight, we could exclude variation in maturation of oocytes ${ }^{34}$. Furthermore we excluded that the variation could be due to polymorphisms in the PCR primers used (Table S3.2). The considerable variation among 137 oocytes from the same eight female (Figure 3.1B) indicated that it was independent of the nuclear genome and we conclude that the variation in mtDNA copy number among oocytes is individual stochastic variation, rather than genetic variation. Such variation within an individual has also been reported in human ${ }^{29}$ and bovine oocytes ${ }^{35}$, which implies that every individual can generate oocytes with either a very low or a very high mtDNA copy number.

\section{mtDNA copy number per cell decreases during embryonic development}

Until the 256-cell stage, the mtDNA copy number per cell in complete zebrafish embryos halved during every cell cycle, while the total amount did not change, consistent with lack of mtDNA replication and degradation. The decrease in mtDNA 
content per cell continued until early segmentation and at these stages about 1654 mtDNA molecules were present per cell. This is in line with findings in mouse, in which no changes in total mtDNA copy number were observed until the blastocyst stage $\left(\sim 128\right.$ cells $\left.^{28}\right)$. When the gastrulation stages pass into the segmentation stages, at which primary organogenesis occurs and the first muscle-driven movements can be observed ${ }^{16}$, mtDNA replication is initiated. During this period, zebrafish embryos display maximum proton leak, which might reflect elevated mitochondrial function ${ }^{36}$. The onset of mtDNA replication most likely reflects a switch to oxidative phosphorylation (OXPHOS) as main energy source, while the large energy requirement during the first developmental stage is provided by glycolysis ${ }^{37}$, a faster energy source ${ }^{36}$.

\section{High variation in the germline mtDNA bottleneck size}

In PGCs, we found a negative correlation between mtDNA copy number and developmental time with a minimal mean mtDNA copy number of 171 (an estimated 193.5 ( \pm 56.3 ) following non-linear regression analysis), with a SD of 111. Since we quantified the mtDNA amount for groups of 80 PGCs, the actual variation among individual cells might be even higher. This high variation in PGCs from 8 hpf to 72 hpf is comparable to mature oocytes and the bottom of the bottleneck seems to be defined by developmental stage and not by the mtDNA copy number. Given the high variation of mtDNA copies in the oocyte, each female can produce PGCs, which, by chance, possess at the bottom only a few mtDNA molecules. At this stage, the risk that de novo mtDNA mutations ${ }^{38}$, reach functional significance will be highest.

The germline bottleneck size of 170-200 mtDNA molecules is close to the value in mice of $\sim 185$, (based on mtDNA heteroplasmy segregation between several generations in the BALB/NZB mouse ${ }^{9}$ ) and $\sim 200$ (based on direct measurements ${ }^{7}$; $\left.{ }^{8}\right)$. In humans, the bottleneck size has been estimated to be $\sim 173$, based on the heteroplasmy distribution among 82 single primary oocytes from a woman carrying the $3243 A>G$ mtDNA mutation ${ }^{39}$. The bottleneck size in zebrafish also resembles the size in cows $\left(65-163^{40}\right)$, salmons $\left(80-88^{11}\right)$ and even crickets $\left(87-395^{40}\right)$. Some studies in humans estimated a lower bottleneck size, for instance a bottleneck size of $\sim 90^{14}$, only $30-35^{13}$, or even $1-5^{12}$. These were calculated from segregation patterns of variants that might not be neutral and selection events could apply ${ }^{41}$. Since most models assume genetic drift only ${ }^{42}$, this would lead to an underestimation of the mtDNA bottleneck size. Nevertheless, the different reported values on the bottleneck size could also reflect large biological variation. Flexibility in the decrease of mtDNA copy number during the bottleneck is in line with a mathematical model for the bottleneck ${ }^{43}$

\section{Increased mtDNA copy number during oocyte maturation}

The mtDNA copy number increased rapidly during oocyte development, with a 20fold difference between stage I oocytes and mature oocytes, indicating that mtDNA replication is a prerequisite for oocyte maturation. Substantial increase in the mtDNA copy number during early oocyte maturation has also been observed in mice ${ }^{10}$. The correlation between the size of the cells and the mtDNA copy number (Figure 3.3B) showed that growth and mtDNA replication were closely connected. During oogenesis, a PGC of $\sim 7 \mu \mathrm{m}$ develops into an oocyte, which grows and reaches a diameter of 140 
$\mu \mathrm{m}$ at the end of stage $\mathrm{I}^{17}$. At this stage variation in mtDNA copy numbers between cells was highest (ranging from $\sim 20.000-3.7 \times 10^{6}$ ) and no fixed oocyte volume per mtDNA could be observed. Probably, at this stage cell growth precedes and might trigger mtDNA replication, whereas during the further developmental stages, it is less clear whether cell growth drives mtDNA replication or vice versa. We failed to measure an increase in mtDNA copy number in PGCs that arrived at the region where the gonads develop, as has been described in mice ${ }^{7 ;}$. This discrepancy might arise because fish oogonia, in contrast to mammals, keep constantly renewing the stocks of young oocytes and follicles. In fish, mitosis in oogonia (starting point of oogenesis) is activated after ovulation in adult zebrafish ${ }^{44}$, while human oogonia undergo mitosis already during weeks 9-22 of embryonic development ${ }^{45}$. Although we were not able to isolate oogonia and the earliest and smallest primary oocytes, the high mtDNA copy numbers in stage I oocytes suggests that the mtDNA replication necessary to achieve high oocyte mtDNA loads occurred later in zebrafish life (in adults) than in mammals (during embryonic development).

\section{A small non-germline mtDNA bottleneck size}

The bottleneck size in non-germline zebrafish cells (non-PGCs) is 50 mtDNA molecules (an estimated $89.4 \pm 16.3$ following non-linear regression analysis) per cell with a downward trend in the mtDNA copy number during development. As non-PGCs can be any cell from the developing embryo, the bottleneck size derived from the whole embryo analysis (16-54 mtDNA copies) confirms this number. The decrease in mtDNA copy number has been suggested to establish a set-point during early embryogenesis, which increases later during development in order to satisfy cellspecific ATP requirements through OXPHOS ${ }^{46}$. During the first $48 \mathrm{hpf}$ we did not observe an increase in the mtDNA copy number of the non-germline cells (Figure 3.1), while after $72 \mathrm{hpf}$ a trend towards higher mtDNA amounts was observed (Figure $3.2 \mathrm{C}$ ). The latter observation could indicate that mtDNA amounts go up once the larvae use their skeletal muscle for free swimming and hunting ${ }^{16}$.

\section{The germline and the non-germline mtDNA bottleneck}

In line with our observation in zebrafish, murine somatic cells have highly variable mtDNA copy numbers during early embryogenesis and the mtDNA content was downregulated from $8 \mathrm{dpc}$ onwards ${ }^{10}$. Furthermore, as in the present study, the mtDNA copy number in murine non-germline cells was lower than in germline cells ${ }^{10}$. Although mtDNA copy number between PGCs and non-PGCs were not statistically different, correlation and non-linear regression analysis (Figure 3.2C) indicated a difference in the course or timing of the mtDNA copy number over development. The increase in mtDNA content in germline cells occurs during oogenesis in adult zebrafish (Figure 3.4), long after the formation of the organ systems (non-germline cells) during zebrafish embryogenesis. This difference in timing, if comparable in humans, could explain differences in segregation of mtDNA heteroplasmic mutations among germline and somatic tissues in humans ${ }^{42}$. Especially in case of familial mtDNA mutations ${ }^{2}$, these segregation and replication mechanisms could explain the large tissue-specific differences in mutation load ${ }^{47}$. This is corroborated by the 
observation that HIV-interventions, which reduce mtDNA copy number can induce a somatic bottleneck, can result in age-related mitochondrial disease due to clonal expansion of preexisting mtDNA mutations ${ }^{48}$.

We have quantified the whole germline mtDNA bottleneck in a single model (Figure 3.4). Mechanistically, both the germline and the non-germline bottlenecks rely on the same principle: division of the mtDNA over the daughter cells during early embryogenesis in the absence of replication, until increased energy requirements during development or oogenesis activate mtDNA replication. However, as the bottleneck in non-germline cells only affects the individual, while segregation in PGCs affects future generations, the germline bottleneck is of higher evolutionary importance. Nevertheless, an understanding of the difference between the bottlenecks would allow a better prediction of the risk an individual has to develop an mtDNA disorder, which is determined by both the germline and the non-germline bottleneck. Due to a clear difference in somatic and germline specification in zebrafish, as well as fast development and easy access to the organs, zebrafish are an attractive model to study these possible tissue-specific mtDNA bottleneck effects in further detail.

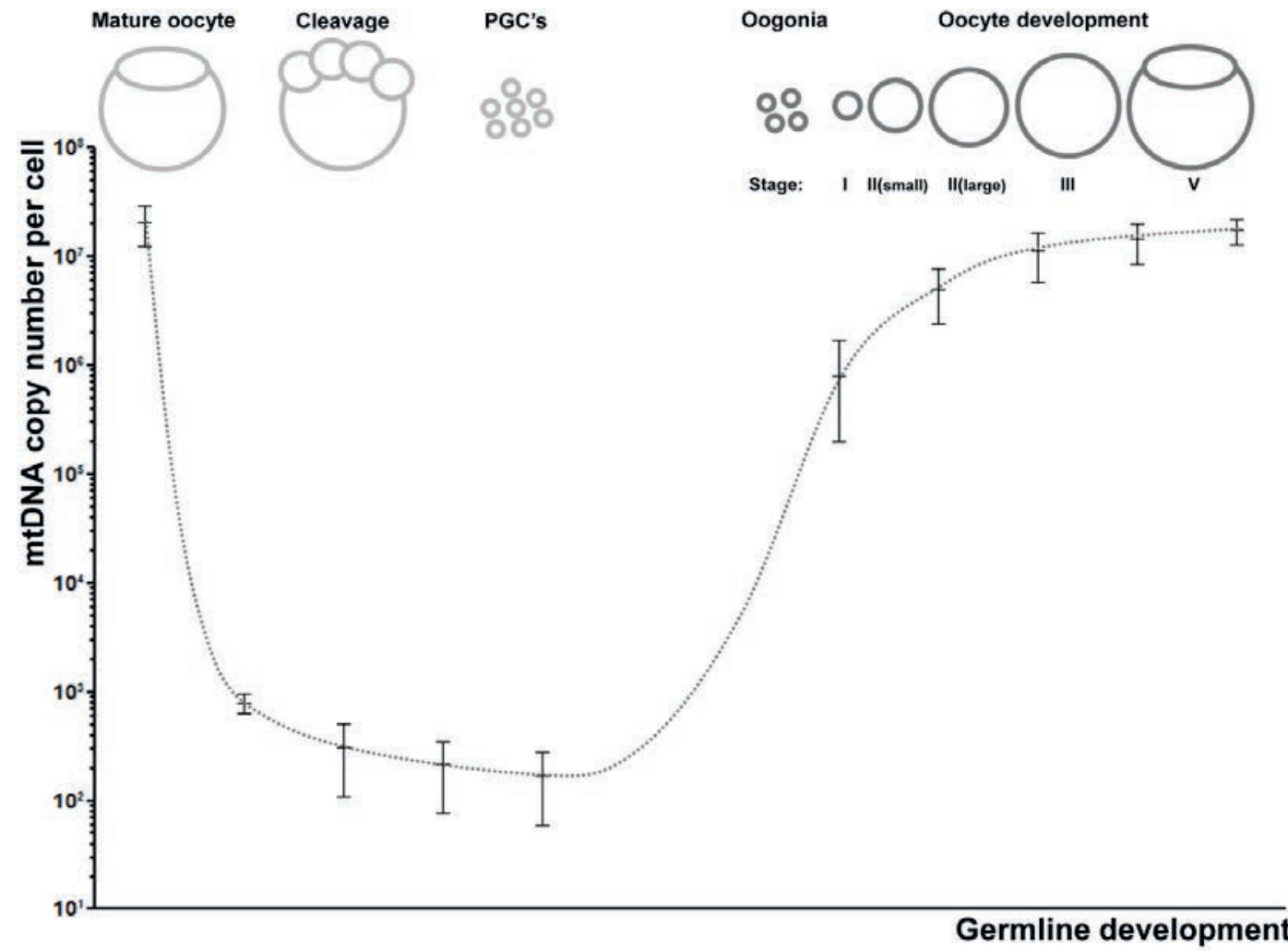

Figure 3.4 | Summary of the germline mtDNA bottleneck. The germline mtDNA bottleneck consists of 1) random partitioning of oocyte mtDNA during the early developmental cell divisions, leading to variable mtDNA bottleneck sizes and 2) extensive mtDNA replication during oogenesis. 


\section{Supplementary data}

Supplementary Figures, Experimental Procedures and tables are available online at http://dx.doi.org/10.1016/j.celrep.2016.06.023

\section{Acknowledgements}

R. Stephan and S. Ormenese (GIGA Flow Cytometry platform, Liège University, Belgium) are acknowledged for their assistance during cell sorting, I. Eijkenboom for generating the $m t-n d 1$ curves, M. Adriaens for reviewing statistical analyses, R. GotoKazeto (Hokkaido University, Japan) for supplying the nanos3 3'UTR pCS2+ vector and Jana Pfeiffer (Münster University, Germany) for assisting in transportation of the transgenic zebrafish. We are grateful for the fellowship of GROW-School for oncology and Developmental Biology to A.B.C.O. and H.J.M.S., to the alma-in-silico project funded by the Interreg IV program of the EC to M.M. and H.J.M.S., the E-RARE 2 project GENOMIT [R 50.02.12F] to M.M. and H.J.M.S. and to the Dutch Princes Beatrix Fonds for their grant to T.E.J.T. and H.J.M.S.. K.T. and E.R. are supported by the European Research Council (ERC), the Deutsche Forschungsgemeintschaf (DFG) and the Cells in Motion cluster of Excellence (EXC 1003-CIM). M.W. and M.M. are grateful to Walloon Region (FEDER. GIGA2 Bioindustry support) and the Fond Nacional de la Recherché Scientifique (F.N.R.S.) with the GENOMIT project. M.G. and H.J.M.S. are grateful for the support of the Dutch Province of Limburg.

\section{References}

1. Lynch, M., Koskella, B., and Schaack, S. (2006). Mutation pressure and the evolution of organelle genomic architecture. Science 311, 1727-1730.

2. Otten, A.B., and Smeets, H.J. (2015). Evolutionary defined role of the mitochondrial DNA in fertility, disease and ageing. Human reproduction update.

3. Lee, H.S., Ma, H., Juanes, R.C., Tachibana, M., Sparman, M., Woodward, J., Ramsey, C., Xu, J., Kang, E.J., Amato, P., et al. (2012). Rapid mitochondrial DNA segregation in primate preimplantation embryos precedes somatic and germline bottleneck. Cell reports 1, 506-515.

4. Sharpley, M.S., Marciniak, C., Eckel-Mahan, K., McManus, M., Crimi, M., Waymire, K., Lin, C.S., Masubuchi, S., Friend, N., Koike, M., et al. (2012). Heteroplasmy of mouse mtDNA is genetically unstable and results in altered behavior and cognition. Cell 151, 333-343.

5. Howell, N., Halvorson, S., Kubacka, I., McCullough, D.A., Bindoff, L.A., and Turnbull, D.M. (1992). Mitochondrial gene segregation in mammals: is the bottleneck always narrow? Human genetics 90, 117-120.

6. Hauswirth, W.W., and Laipis, P.J. (1982). Mitochondrial DNA polymorphism in a maternal lineage of Holstein cows. Proceedings of the National Academy of Sciences of the United States of America 79, 4686-4690.

7. Cree, L.M., Samuels, D.C., de Sousa Lopes, S.C., Rajasimha, H.K., Wonnapinij, P., Mann, J.R., Dahl, H.H., and Chinnery, P.F. (2008). A reduction of mitochondrial DNA molecules during embryogenesis explains the rapid segregation of genotypes. Nature genetics 40, 249-254.

8. Wai, T., Teoli, D., and Shoubridge, E.A. (2008). The mitochondrial DNA genetic bottleneck results from replication of a subpopulation of genomes. Nature genetics 40, 1484-1488.

9. Jenuth, J.P., Peterson, A.C., Fu, K., and Shoubridge, E.A. (1996). Random genetic drift in the female germline explains the rapid segregation of mammalian mitochondrial DNA. Nature genetics $14,146-151$. 
10. Cao, L., Shitara, H., Horii, T., Nagao, Y., Imai, H., Abe, K., Hara, T., Hayashi, J., and Yonekawa, $\mathrm{H}$. (2007). The mitochondrial bottleneck occurs without reduction of mtDNA content in female mouse germ cells. Nature genetics 39, 386-390.

11. Wolff, J.N., White, D.J., Woodhams, M., White, H.E., and Gemmell, N.J. (2011). The strength and timing of the mitochondrial bottleneck in salmon suggests a conserved mechanism in vertebrates. PloS one 6, e20522.

12. Marchington, D.R., Hartshorne, G.M., Barlow, D., and Poulton, J. (1997). Homopolymeric tract heteroplasmy in mtDNA from tissues and single oocytes: support for a genetic bottleneck. American journal of human genetics 60 , 408-416.

13. Rebolledo-Jaramillo, B., Su, M.S., Stoler, N., McElhoe, J.A., Dickins, B., Blankenberg, D., Korneliussen, T.S., Chiaromonte, F., Nielsen, R., Holland, M.M., et al. (2014). Maternal age effect and severe germ-line bottleneck in the inheritance of human mitochondrial DNA. Proceedings of the National Academy of Sciences of the United States of America 111, 1547415479.

14. Pallotti, F., Binelli, G., Fabbri, R., Valentino, M.L., Vicenti, R., Macciocca, M., Cevoli, S., Baruzzi, A., DiMauro, S., and Carelli, V. (2014). A wide range of 3243A>G/tRNALeu(UUR) (MELAS) mutation loads may segregate in offspring through the female germline bottleneck. PloS one 9 , e96663.

15. Goto-Kazeto, R., Saito, T., Takagi, M., Arai, K., and Yamaha, E. (2010). Isolation of teleost primordial germ cells using flow cytometry. The International journal of developmental biology 54, 1487-1492.

16. Kimmel, C.B., Ballard, W.W., Kimmel, S.R., Ullmann, B., and Schilling, T.F. (1995). Stages of embryonic development of the zebrafish. Developmental dynamics : an official publication of the American Association of Anatomists 203, 253-310.

17. Selman, K., Wallace, R.A., Sarka, A., and Qi, X. (1993). Stages of oocyte development in the zebrafish, Brachydanio rerio. Journal of Morphology 218, 203-224.

18. Otten, A.B., Stassen, A.P., Adriaens, M., Gerards, M., Dohmen, R.G., Timmer, A.J., Vanherle, S.J., Kamps, R., Boesten, I.B., Vanoevelen, J.M., et al. (2016). Zebrafish oocytes with a low mtDNA copy number are at risk of de novo mtDNA point mutations reaching functional significance (chapter 4). Thesis, Auke Otten.

19. Paksa, A., and Raz, E. (2015). Zebrafish germ cells: motility and guided migration. Current opinion in cell biology $36,80-85$.

20. Wang, R.L., Bencic, D.C., Garcia-Reyero, N., Perkins, E.J., Villeneuve, D.L., Ankley, G.T., and Biales, A.D. (2014). Natural Variation in Fish Transcriptomes: Comparative Analysis of the Fathead Minnow (Pimephales promelas) and Zebrafish (Danio rerio). PloS one 9, e114178.

21. Artuso, L., Romano, A., Verri, T., Domenichini, A., Argenton, F., Santorelli, F.M., and Petruzzella, V. (2012). Mitochondrial DNA metabolism in early development of zebrafish (Danio rerio). Biochimica et biophysica acta 1817, 1002-1011.

22. Wolff, J.N., and Gemmell, N.J. (2008). Lost in the zygote: the dilution of paternal mtDNA upon fertilization. Heredity 101, 429-434.

23. Zeng, H.T., Yeung, W.S., Cheung, M.P., Ho, P.C., Lee, C.K., Zhuang, G.L., Liang, X.Y., and O, W.S. (2009). In vitro-matured rat oocytes have low mitochondrial deoxyribonucleic acid and adenosine triphosphate contents and have abnormal mitochondrial redistribution. Fertility and sterility 91, 900-907.

24. Iwata, H., Goto, H., Tanaka, H., Sakaguchi, Y., Kimura, K., Kuwayama, T., and Monji, Y. (2011). Effect of maternal age on mitochondrial DNA copy number, ATP content and IVF outcome of bovine oocytes. Reproduction, fertility, and development 23, 424-432.

25. Duran, H.E., Simsek-Duran, F., Oehninger, S.C., Jones, H.W., Jr., and Castora, F.J. (2011). The association of reproductive senescence with mitochondrial quantity, function, and DNA integrity in human oocytes at different stages of maturation. Fertility and sterility 96, 384-388.

26. Murakoshi, Y., Sueoka, K., Takahashi, K., Sato, S., Sakurai, T., Tajima, H., and Yoshimura, Y. (2013). Embryo developmental capability and pregnancy outcome are related to the mitochondrial DNA copy number and ooplasmic volume. Journal of assisted reproduction and genetics 30, 1367-1375.

27. Cotterill, M., Harris, S.E., Collado Fernandez, E., Lu, J., Huntriss, J.D., Campbell, B.K., and Picton, H.M. (2013). The activity and copy number of mitochondrial DNA in ovine oocytes 
throughout oogenesis in vivo and during oocyte maturation in vitro. Molecular human reproduction 19, 444-450.

28. Ebert, K.M., Liem, H., and Hecht, N.B. (1988). Mitochondrial DNA in the mouse preimplantation embryo. Journal of reproduction and fertility 82, 145-149.

29. Reynier, P., May-Panloup, P., Chretien, M.F., Morgan, C.J., Jean, M., Savagner, F., Barriere, P., and Malthiery, Y. (2001). Mitochondrial DNA content affects the fertilizability of human oocytes. Molecular human reproduction 7, 425-429.

30. Santos, T.A., El Shourbagy, S., and St John, J.C. (2006). Mitochondrial content reflects oocyte variability and fertilization outcome. Fertility and sterility 85, 584-591.

31. May-Panloup, P., Chretien, M.F., Jacques, C., Vasseur, C., Malthiery, Y., and Reynier, P. (2005). Low oocyte mitochondrial DNA content in ovarian insufficiency. Human reproduction 20, 593597.

32. Wai, T., Ao, A., Zhang, X., Cyr, D., Dufort, D., and Shoubridge, E.A. (2010). The role of mitochondrial DNA copy number in mammalian fertility. Biology of reproduction 83, 52-62.

33. Fragouli, E., Spath, K., Alfarawati, S., Kaper, F., Craig, A., Michel, C.E., Kokocinski, F., Cohen, J., Munne, S., and Wells, D. (2015). Altered levels of mitochondrial DNA are associated with female age, aneuploidy, and provide an independent measure of embryonic implantation potential. PLoS genetics 11, e1005241.

34. Jansen, R.P., and de Boer, K. (1998). The bottleneck: mitochondrial imperatives in oogenesis and ovarian follicular fate. Molecular and cellular endocrinology 145, 81-88.

35. Cree, L.M., Hammond, E.R., Shelling, A.N., Berg, M.C., Peek, J.C., and Green, M.P. (2015). Maternal age and ovarian stimulation independently affect oocyte mtDNA copy number and cumulus cell gene expression in bovine clones. Human reproduction 30, 1410-1420.

36. Stackley, K.D., Beeson, C.C., Rahn, J.J., and Chan, S.S. (2011). Bioenergetic profiling of zebrafish embryonic development. PloS one 6, e25652.

37. Facucho-Oliveira, J.M., and St John, J.C. (2009). The relationship between pluripotency and mitochondrial DNA proliferation during early embryo development and embryonic stem cell differentiation. Stem cell reviews 5, 140-158.

38. Thorburn, D.R. (2004). Mitochondrial disorders: prevalence, myths and advances. Journal of inherited metabolic disease 27, 349-362.

39. Brown, D.T., Samuels, D.C., Michael, E.M., Turnbull, D.M., and Chinnery, P.F. (2001). Random genetic drift determines the level of mutant mtDNA in human primary oocytes. American journal of human genetics $68,533-536$.

40. Rand, D.M., and Harrison, R.G. (1986). Mitochondrial DNA transmission genetics in crickets. Genetics 114, 955-970.

41. Stewart, J.B., Freyer, C., Elson, J.L., Wredenberg, A., Cansu, Z., Trifunovic, A., and Larsson, N.G. (2008). Strong purifying selection in transmission of mammalian mitochondrial DNA. PLoS biology 6 , e10.

42. Wonnapinij, P., Chinnery, P.F., and Samuels, D.C. (2008). The distribution of mitochondrial DNA heteroplasmy due to random genetic drift. American journal of human genetics 83, 582-593.

43. Johnston, I.G., Burgstaller, J.P., Havlicek, V., Kolbe, T., Rulicke, T., Brem, G., Poulton, J., and Jones, N.S. (2015). Stochastic modelling, Bayesian inference, and new in vivo measurements elucidate the debated mtDNA bottleneck mechanism. eLife 4, e07464.

44. Jalabert, B. (2005). Particularities of reproduction and oogenesis in teleost fish compared to mammals. Reproduction, nutrition, development 45, 261-279.

45. Jones, R.E. (1997). Human Reproductive Biology.(San Diego: Academic Press, Elsevier).

46. Facucho-Oliveira, J.M., Alderson, J., Spikings, E.C., Egginton, S., and St John, J.C. (2007). Mitochondrial DNA replication during differentiation of murine embryonic stem cells. Journal of cell science 120, 4025-4034.

47. St John, J.C. (2012). Transmission, inheritance and replication of mitochondrial DNA in mammals: implications for reproductive processes and infertility. Cell and tissue research 349 , 795-808.

48. Payne, B.A., Wilson, I.J., Hateley, C.A., Horvath, R., Santibanez-Koref, M., Samuels, D.C., Price, D.A., and Chinnery, P.F. (2011). Mitochondrial aging is accelerated by anti-retroviral therapy through the clonal expansion of mtDNA mutations. Nature genetics $43,806-810$. 



\title{
Chapter 4
}

\section{Zebrafish oocytes with a low mtDNA copy}

\author{
number are at risk of de novo mtDNA
}

point mutations reaching functional

significance

Auke BC Otten, Alphons PM Stassen, Michiel Adriaens, Mike Gerards, Richard GJ Dohmen, Adriana J Timmer, Sabina JV Vanherle, Rick Kamps, Iris BW Boesten, Jo M Vanoevelen, Marc Muller, Hubert JM Smeets 


\section{Summary}

Of all pathogenic mitochondrial DNA (mtDNA) mutations in humans, $\sim 25 \%$ is de novo, although the de novo occurrence in oocytes has never been directly assessed. We used next generation sequencing to detect mutations directly in the mtDNA of 13-15 individual oocytes and in three somatic tissues from eight zebrafish females. Various statistical and biological filters allowed reliable detection of (de novo) variants with heteroplasmy $\geq 1.5 \%$. In total, 38 de novo mutations were detected in 19 of 103 oocytes, indicating that $20 \%$ of the oocytes carry at least one de novo mutation with heteroplasmy $\geq 1.5 \%$. This frequency of de novo mutations is close to that deducted from the reported error rate of polymerase gamma, the mitochondrial replication enzyme, implying that errors made during mtDNA replication are a likely explanation. Substantial variation in the mutation prevalence among oocytes can be explained by the highly variable mtDNA copy number, since we previously reported that $\sim 20 \%$ of the germ cells have a mtDNA copy number of $\leq 73$ and would lead to detectable mutation loads. In conclusion, replication errors made during oogenesis are an important source of de novo mtDNA mutations and their location and heteroplasmy level determine the eventual significance.

\section{Introduction}

Comparative sequence analysis of the mitochondrial DNA (mtDNA) has revealed a high degree of variability, much higher than its nuclear counterpart ${ }^{1}$. This is generally explained by limited recombination and recombination-mediated mtDNA repair events to counteract errors made during mtDNA replication ${ }^{2}$ and the close proximity of the unprotected mtDNA to the oxidative phosphorylation (OXPHOS) machinery, which produces (potentially) mutagenic reactive oxygen species (ROS) ${ }^{3}$. As a result, mtDNA mutations are an important cause of a group of devastating inherited diseases ${ }^{4}$. To date, over 150 pathogenic mtDNA mutations have been identified as well as many more polymorphisms of unknown significance ${ }^{5}$. As human and animal cells have a high mtDNA copy number, wild-type and variant mtDNA genotypes can co-exist, a state referred to as heteroplasmy. The high mtDNA copy number compensates low-level pathogenic mtDNA mutations and avoids disease manifestation. A pathogenic mtDNA mutation will only manifest if its heteroplasmy value exceeds a certain threshold ${ }^{5}$. The mtDNA inherits maternally, and a female carrying an mtDNA mutation can transmit this mutation to her offspring through the mtDNA of her oocytes.

Maternal inheritance of pre-existing mtDNA mutation does not explain all patients suffering for mtDNA mutations. In $25 \%$ of these patients, the disease-causing mutation cannot be detected in the maternal mtDNA (Sallevelt et al., in preparation). Although we cannot exclude some of these mutation were present at undetectable low-level heteroplasmy levels in the maternal mtDNA, this suggests that most of these mutations have occurred de novo during germ line development. The inheritance of the mtDNA occurs through a segregational bottleneck: only a limited number of the mtDNA molecules, the so-called bottleneck size, from the oocyte are transmitted to the germline cells of the next generation ${ }^{6}$. We hypothesize that, in case of a constant mutation rate, a low mtDNA copy number at the bottom of the bottleneck could lead 
to de novo mutations reaching detectable heteroplasmy levels, which dependent on the nature of the mutations can be of functional and/or pathogenic significance. Experimental evidence for this hypothesis has long been difficult to obtain, because of the low heteroplasmy level at which these mutations occur, which were generally below the detection level of conventional sequencing techniques. Over the past decade, next-generation sequencing (NGS) technologies have been developed, allowing an in depth determination of the mutations and heteroplasmy levels directly in the mtDNA of individual oocytes.

An estimation of the prevalence of de novo mtDNA mutation requires a significant number of oocytes to be sequenced. For both biological and ethical reason this is difficult to achieve in humans and most animals. In contrast, oocyte collection from zebrafish is easy and efficient and therefore we used zebrafish oocytes to assess the de novo mtDNA mutation risk. We characterized de novo mutations, their location and their heteroplasmy level in 103 oocytes and three somatic (maternal) tissues from eight different female zebrafish using NGS with a minimal coverage of 1,700. Furthermore, for all oocytes with one or more de novo mutation(s), we estimated the mtDNA copy number at which these mutations arose, based on their heteroplasmy levels. Given the high mtDNA sequence similarity between humans and zebrafish ${ }^{7}$ and the conservation of the mtDNA bottleneck within the animal kingdom ${ }^{6 ; 8-10}$, our findings have insinuations for the occurrence of de novo mtDNA disease in humans.

\section{Material and Methods}

\section{Zebrafish maintenance and sample collection}

Wild-type female zebrafish from the $A B$ strain were used. Raising and housing was according to standard procedures at $28^{\circ} \mathrm{C}^{11}$ in the zebrafish facility of Liège University, where local ethical approval by the committee of Animal Research was obtained. Mature unfertilized oocytes were collected by squeezing the abdomen of anaesthetized females. The oocytes used were apparently normal in morphological appearance. After oocyte collection, the female fish were sacrificed in ice-cold water, after which biopsies of brain, liver and muscle were obtained. An overview of the 127 samples is given in Table 4.1.

Table 4.1 | Overview of samples collected

\begin{tabular}{|l|l|l|l|l|l|l|l|l|}
\hline Zebrafish \# & 1 & 2 & 3 & 4 & 5 & 6 & 7 & 8 \\
\hline Biopsies & 3 & 3 & 3 & 3 & 3 & 3 & 3 & 3 \\
\hline $\begin{array}{l}\text { Number of } \\
\text { oocytes }\end{array}$ & 3 & 13 & 15 & 14 & 15 & 14 & 15 & 14 \\
\hline
\end{tabular}

\section{Isolation procedure of mtDNA}

Oocytes were collected in sterile tubes and lysed for 4 hours at $50^{\circ} \mathrm{C}$ in $500 \mu$ DNA lysis buffer containing $75 \mathrm{mM} \mathrm{NaCl}, 50 \mathrm{mM}$ EDTA, $20 \mathrm{mM}$ HEPES, $0.4 \%$ SDS and 
$200 \mu \mathrm{g}$ proteinase K (Sigma). Subsequently, isopropanol was added and samples were precipitated overnight at $-20^{\circ} \mathrm{C}$. After thorough centrifugation, the DNA pellet was washed with $70 \%$ ethanol and dissolved in TE buffer. The biopsies of brain, liver and muscle from the adult female fish were collected in sterile tubes containing Nuclei Lysis solution from the Wizard Genomic Purification Kit (Promega). Subsequently, mtDNA was extracted according to manufacturer's instructions and dissolved in FG3buffer.

\section{mtDNA amplification and sequencing}

The mtDNA (reference NCBI: NC002333.2) was amplified in three $\sim 5.6 \mathrm{~kb}$ amplicons (A-C). Fragment A (Forward: CACACCCCTGACTCCCAAAG, Reverse: GGTCGTTTGTACCCGTCAGT) amplified a target spanning nucleotide 16,594 (gene: trna-pro) to 5,952 (nd2), fragment B (Forward: AAATTAACACCCTAACAACGACCTG, Reverse: GGGGATCAGTACTTTTAGCATTGTAGT) an amplicon from nucleotide 5,669 (nd2) to 11,319 (nd4) and fragment $C$ amplified the mtDNA from nucleotide 11,170 (nd4l) to 295 (D-loop). Primers (designed with Primer3) were specific for the mtDNA to avoid the amplification of nuclear-encoded mitochondrial pseudogenes. PCR amplification was performed using Phusion Hot Start II DNA polymerase in GC-buffer (ThermoScientific): 30 s at $98^{\circ} \mathrm{C}$, followed by 40 cycles of $10 \mathrm{~s}$ at $98^{\circ} \mathrm{C}$ (denaturation), 20s at $58^{\circ} \mathrm{C}$ (annealing) and $8 \mathrm{~min}$ at $72^{\circ} \mathrm{C}$ (extension), with a final step for $10 \mathrm{~min}$ at $72^{\circ} \mathrm{C}$. Amplification was checked using electrophoresis on a $1 \%$ agarose gel. Amplicons were purified using the Agencourt AMPure XP system (Beckman-Coulter), according to the protocol of the manufacturer. Subsequently, the three purified amplicons were (equimolar) mixed and processed using the customized Nextera XT protocol ${ }^{12}$. The library of a random subset of the samples was analysed using a Bioanalyzer 2100 High Sensitivity DNA chip (Agilent Technologies) to confirm quantity and size of the library. Libraries were indexed and 18 libraries were pooled per lane and analysed on the HiSeq 2000 system (Illumina), using a read length of 1000 base pairs. PhiX (1\%) was spiked in every lane as an internal control.

\section{Pre-processing of Next-Generation sequencing data}

As the mtDNA is a multicopy genome (resulting in many biological duplicates), duplicate reads were included in the analysis. Demultiplexing of the data was performed using Illumina CASAVA software (v.1.8.2) and reads were aligned against the mitochondrial reference sequence for the zebrafish (NCBI: NC002333.2) using Burrows-Wheeler Alligner (BWA) software (v.0.5.9) ${ }^{13}$. For variant calling, we used Phyton 2.6.6., Phyton Package pysam 0.7.8 and SAMTools 0.1.19. In-house built Perl tools were used to process the variants. As the prevalence of any of the four nucleotides per position was counted to call a variant, the heteroplasmy value was calculated as the ratio of one of the nucleotides over the coverage, which was defined as the total count of any nucleotide at a certain position.

\section{Identification of de novo mutations from point heteroplasmic sites}

A statistical algorithm was developed to distinguish variant calls from the noise signal, as well as to determine whether a variant reported in an oocyte was absent in the corresponding female fish, and thus arose de novo. A call from a sample is included in 
the analysis if its coverage is above the threshold, which is determined by calculating the median coverage for every position of the mtDNA genome, based on the coverage data of all 127 samples (Figure 4.1). Assuming sequencing quality is independent of the nucleotide position, the median value is the most robust estimate of the coverage across the entire mtDNA genome. This implies that the lowest median coverage value is a robust estimate of the minimal reliable coverage and of the maximum background (noise) signal. As the lowest median coverage value was $\sim 1,700$ (Figure 4.1A), this was chosen as a cut-off value for a position to be included, preventing variants with lower coverage from influencing the statistical calculations we applied to exclude false positives.

To discriminate false positives in the higher coverage group from true variants we compared the percentage heteroplasmy of the variant nucleotide of a particular sample with the average percentage heteroplasmy of all other samples (female tissues and oocytes, but without littermate oocytes and tissues from the mother). To this end, for each variant a probability distribution of the heteroplasmy values was generated (using all samples) and transformed to a Gaussian distribution using 'a rank-transformation'. We assumed this distribution to be a representation of the noise signal for this variant, inherent to the procedure ${ }^{14}$. Hence, we calculated a $z$-score and $P$-value (one tailed) and considered variants with a $P$-value $\leq 0.01$ as true variants statistically different from the noise signal. The check for de novo variants was only performed for variants for which the coverage of the oocyte and of $\geq 2$ tissues of its mother was $\geq 1,700$. Finally, a variant was assumed de novo if the $P$-value of this variant in the oocyte was $\leq 0.01$, while in the tissues from the corresponding female the $P$-value was $>0.01$ (= absent or in noise signal) and if the heteroplasmy percentage was $\geq 1.0 \%$.

A



B

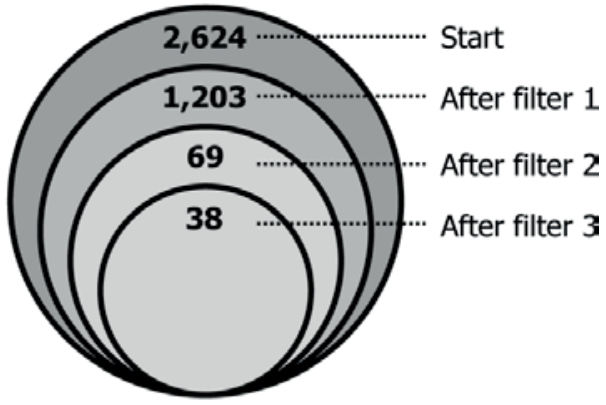

Figure 4.1 | Median reads per position and filtering steps used to determine de novo mutations. A) Overview of the median reads per position of the mtDNA genome (red line) across all samples. The blue line indicates the threshold value for the coverage value to include samples. B) Results of filtering steps performed to determine de novo mutations. All reads with coverage above $1,700 x(1,592,714$ nucleotides) were statistically analysed, resulting in 2,624 variants with a $P$-value $\leq 0.01$. Subsequent filter steps performed: 1) prevalence tissues adult female zebrafish $<1.0 \%$, 2) heteroplasmy difference between tissues from adult zebrafish and her oocytes (O-M) $\geq 1.5 \%$ and 3 ) mutations not detected in the two nucleotides adjacent (upstream or downstream) of another variant. After filtering, 38 de novo mutations remained. 


\section{Estimation of mtDNA copy number and mutation rate}

From the average heteroplasmy value (het-\%) of all de novo mutations within one oocyte, we estimated the mtDNA copy number at the time the mutation occurred. Assuming only one copy is mutated and heteroplasmy values stay the same during oocyte development, than the mtDNA copy number $(z)$ at the time the mutation occurred can be calculated using: $z=100 /$ het- $\%$. Subsequently, we aimed to estimate the mutation rate for mutations $>1.5 \%$. To do this, we first calculated for every oocyte the total number of detectable nucleotides $(y)$ that were present at the time the mutation arose. Therefore, we multiplied the estimated mtDNA copy number ( $z$ ) with the number of nucleotides that had coverage $>1,700: y=z$ * [\#nucleotides $>1,700$ coverage]. The mutation rate $(x)$ was then calculated by dividing the number of de novo mutations in an oocyte by the total number of analysable nucleotides: $x=$ [\#mutations detected] / $y$.

\section{Results}

We studied the occurrence of detectable de novo mutations in 103 oocytes derived from eight different female zebrafish. A total of 1,592,714 different nucleotide positions had a sequence-coverage $>1,700$. In total, 2,624 different variants were statistically different from the sequencing noise signal and absent in all three maternal tissues and therefore considered as potential de novo mutations. To increase the reliability of our de novo mutation detection, we applied three additional biological filter steps (Figure 4.1B). 1) All oocyte variants for which the heteroplasmy level in one of the corresponding maternal tissues was $\geq 1.0 \%$ were considered to be pre-existing and were therefore excluded. 2) The heteroplasmy level of the variants was corrected for the noise signal by subtracting the maternal $(\mathrm{M})$ heteroplasmy level (=the average of the heteroplasmy level in the three maternal tissues) from the oocyte $(\mathrm{O})$ heteroplasmy level $(\mathrm{O}-\mathrm{M})$ and variants with small $\mathrm{O}-\mathrm{M}$ values $(<1.5 \%)$ were excluded (205 variants had an O-M value of $1.0-1.5 \%$ and 929 variants of $<1.0 \%$ ). This filter restricted our analysis to de novo mutations with a mutation load $\geq 1.5 \%$. 3) A de novo variant was rejected if, within the same oocyte, another variant was detected in the two nucleotides adjacent (upstream or downstream) to this variant, as variants located in close proximity to each other are most likely the result of alignment artefacts ${ }^{15}$. By applying these filters, 38 mutations were considered to be de novo (Table 4.2), of which 36 were unique, as two mutations ( $\mathrm{m} .7247 \mathrm{~T}>\mathrm{G}$ and $\mathrm{m} .10578 \mathrm{C}>\mathrm{A}$ ) were found in two oocytes isolated from the same zebrafish. The 38 de novo mutations were detected in only 19 different oocytes (Table 4.2, indicating that the majority (82\%) did not have a detectable de novo mutation. In three oocytes (5.2, 5.10 and 7.9) more than three de novo point mutations were found. No de novo mutations were detected in any oocyte from zebrafish 6 . The heteroplasmy level of all the de novo mutations ranged from $1.5 \%$ to $9.0 \%$ with an average of $2.7 \%$.

For every oocyte with at least one de novo mutation, we estimated the mtDNA copy number present at the time the mutation occurred. This number ranged from 18 to 67 (Table 4.3). Based on this, we calculated the mutation rate for these oocytes (Table 4.3). For most oocytes this mutation rate was in the range of $10^{-6}$ mutations per 
nucleotide, while oocytes $4.4,5.10$ and 7.3 had a higher mutation rate. On average, the mutation rate in these 19 oocytes was $4.3 \times 10^{-6}$ mutations per nucleotide. Strikingly, for oocyte 8.6 the heteroplasmy values of the two reported de novo mutations greatly differed from each other, resulting in two different estimations of the mutation rate.



Figure 4.2 | Prevalence of de novo variants. Prevalence A) per gene type (protein coding genes, tRNA, rRNA genes and D-loop) and B) per protein-coding gene. The observed number of variants in all oocytes is expressed as the number of variants per nucleotide. If a de novo mutation occurred multiple times, it is only counted once in the construction of these distributions.

The number of unique de novo mutations per base was assessed for every gene (Figure 4.2). In case the de novo mutations were classified per gene function (tRNA genes, rRNA genes, protein coding or D-loop separately), the prevalence of de novo mutations appeared to be slightly higher in the D-loop (4 variants per 1000 bases). 26 variants were in protein-coding genes (ND1, ND4, ND5, ND6, COI, COIII and $c y t-b)$, with little difference in the prevalence among the different protein-coding genes. Four of these mutations were synonymous and 22 non-synonymous, including four mutations leading to a premature stop codon (Table 4.2). 
de novo mtDNA point mutation occurence during oogenesis

Table 4.2 | Heteroplasmic mtDNA de novo mutations in all oocytes

\begin{tabular}{|c|c|c|c|c|}
\hline Oocyte ID & $\begin{array}{c}\text { Corr. Het-\% } \\
\text { (O-M) }\end{array}$ & Gene location & Nucleotide change & Am. Acid change \\
\hline 1.1 & $2.5 \%$ & ND1 & $\mathrm{m} .4164 \mathrm{C}>\mathrm{A}$ & Ser $>$ STOP \\
\hline 2.3 & $1.8 \%$ & $\mathrm{COI}$ & m.6489T>C & Phe $>$ Ser \\
\hline 2.6 & $5.4 \%$ & D-loop & $\mathrm{m} .250 \mathrm{C}>\mathrm{T}$ & - \\
\hline 2.6 & $5.9 \%$ & COII & $m .8700 G>A$ & Val>Lys \\
\hline 2.10 & $1.6 \%$ & $\mathrm{COI}$ & $\mathrm{m} .6510 \mathrm{~T}>\mathrm{A}$ & Val>Glu \\
\hline 3.2 & $3.3 \%$ & D-loop & $\mathrm{m} .283 \mathrm{G}>\mathrm{T}$ & - \\
\hline 3.2 & $1.9 \%$ & ND5 & $\mathrm{m} 14296 \mathrm{~T}>\mathrm{A}$ & lle >Asn \\
\hline 3.9 & $4.1 \%$ & $t R N A-T r p$ & m.6089G $>A$ & - \\
\hline 3.15 & $1.9 \%$ & $\begin{array}{l}N D 4 L \\
N D 4\end{array}$ & m.11303T>A & $\begin{array}{l}\text { Stop }>\text { Lys } \\
\text { Leu>GIn }\end{array}$ \\
\hline 4.4 & $3.1 \%$ & D-loop & $\mathrm{m} .1818 \mathrm{~A}>\mathrm{T}$ & - \\
\hline 4.10 & $1.9 \%$ & D-loop & m.532G $>A$ & - \\
\hline 4.10 & $2.1 \%$ & ND4 & m.11708G >A & Trp>STOP \\
\hline 5.2 & $2.7 \%$ & ND1 & $\mathrm{m} .4077 \mathrm{G}>\mathrm{A}$ & Trp >STOP \\
\hline 5.2 & $2.2 \%$ & $\mathrm{COI}$ & $\mathrm{m} .7112 \mathrm{C}>\mathrm{T}$ & Leu>Phe \\
\hline 5.2 & $1.6 \%$ & $\mathrm{COI}$ & $\mathrm{m} .7247 \mathrm{~T}>\mathrm{G}$ & Trp> Gly \\
\hline 5.2 & $2.2 \%$ & $\mathrm{COI}$ & $\mathrm{m} .7574 \mathrm{G}>\mathrm{T}$ & Gly>Trp \\
\hline 5.2 & $2.2 \%$ & $\mathrm{COI}$ & m.7580G $>A$ & Val>Met \\
\hline 5.3 & $1.8 \%$ & ND6 & m.14894TA & Leu>Phe \\
\hline 5.10 & $1.6 \%$ & 12s rRNA & $m .1220 A>G$ & - \\
\hline 5.10 & $2.4 \%$ & $\mathrm{COI}$ & $\mathrm{m} .7112 \mathrm{C}>\mathrm{T}$ & Leu>Phe \\
\hline 5.10 & $2.1 \%$ & $\mathrm{COI}$ & $\mathrm{m} .7247 \mathrm{~T}>\mathrm{G}$ & Trp> Gly \\
\hline 5.10 & $1.9 \%$ & $\mathrm{COI}$ & m.7253A>G & Met $>$ Val \\
\hline 5.10 & $1.8 \%$ & COIII & m.9909C>A & Arg $>$ STOP \\
\hline 5.10 & $1.6 \%$ & ND4 & m.11500G >C & Val>Leu \\
\hline 5.10 & $1.5 \%$ & tRNA-Leu & m.12838T>G & - \\
\hline 5.10 & $1.6 \%$ & ND5 & $\mathrm{m} .13472 \mathrm{C}>\mathrm{T}$ & Tyr $>$ Tyr \\
\hline 5.13 & $8.2 \%$ & ND6 & m.14761G>A & Leu>Leu \\
\hline 7.3 & $1.5 \%$ & $16 s$ rRNA & $\mathrm{m} .2632 \mathrm{~A}>\mathrm{G}$ & - \\
\hline 7.8 & $1.7 \%$ & $16 s$ rRNA & $\mathrm{m} .2537 \mathrm{~A}>\mathrm{G}$ & - \\
\hline 7.9 & $2.5 \%$ & $12 s r R N A$ & m.1550G >C & - \\
\hline 7.9 & $1.8 \%$ & ND4 & m.12263T>G & Leu>Arg \\
\hline 7.9 & $1.6 \%$ & ND5 & m.13205T>G & Phe>Leu \\
\hline 7.9 & $1.6 \%$ & CytB & m.16232A>C & Thr>Pro \\
\hline 7.9 & $1.5 \%$ & CytB & m.16324A $>T$ & Gly>Gly \\
\hline 8.5 & $2.8 \%$ & ND5 & m.14400C>T & Leu>Leu \\
\hline 8.6 & $9.0 \%$ & $t R N A-G / y$ & m.10578C>A & - \\
\hline 8.6 & $1.5 \%$ & ND4 & m.12464G>T & Trp>Leu \\
\hline 8.10 & $4.6 \%$ & $t R N A-G l y$ & m.10578C>A & - \\
\hline
\end{tabular}

Corr. Het-\%, Oocyte heteroplasmy value corrected from noise signal by subtracting the average heteroplasmy of the corresponding female tissues from the heteroplasmy value detected in the oocyte. Am. Acid change, change in amino acid due to change in the codon sequence. 
Table 4.3 | mtDNA copy number and de novo mutation rate $\geq 1.5 \%$ at a given nucleotide for all oocytes in which at least one de novo mutation was detected

\begin{tabular}{|c|c|c|c|c|c|c|}
\hline $\begin{array}{l}\text { Oocyte } \\
\text { ID }\end{array}$ & $\begin{array}{l}\text { \# de novo } \\
\text { mutations }\end{array}$ & $\begin{array}{l}\text { AverageHet-\% } \\
\text { of de novo } \\
\text { mutation }\end{array}$ & $\begin{array}{c}\text { Copy } \\
\text { number } \\
\text { from het } \%\end{array}$ & $\begin{array}{l}\text { Nucleotides } \\
\text { analysed } \\
\text { (coverage } \\
>1700)\end{array}$ & $\begin{array}{c}\text { Analysable } \\
\text { nucleotides } \\
\text { when mutation } \\
\text { occurred }\end{array}$ & $\begin{array}{c}\text { Mutation rate } \\
(>1.5 \%) \text { per } \\
\text { nucleotide }\end{array}$ \\
\hline 1.1 & 1 & 2.5 & 40 & 05,643 & 225,720 & $4.4 \times 10^{-6}$ \\
\hline 2.3 & 1 & 1.8 & 56 & 16,532 & 925,792 & $1.1 \times 10^{-6}$ \\
\hline 2.6 & 2 & 5.7 & 18 & 16,580 & 298,440 & $6.7 \times 10^{-6}$ \\
\hline 2.10 & 1 & 1.6 & 63 & 14,543 & 916,209 & $1.1 \times 10^{-6}$ \\
\hline 3.2 & 2 & 1.9 & 53 & 16,532 & 876,196 & $2.3 \times 10^{-6}$ \\
\hline 3.9 & 1 & 4.1 & 24 & 16,555 & 397,320 & $2.5 \times 10^{-6}$ \\
\hline 3.15 & 1 & 1.9 & 53 & 14,902 & 789,806 & $1.3 \times 10^{-6}$ \\
\hline 4.4 & 1 & 3.1 & 32 & 02,816 & 90,112 & $1.1 \times 10^{-5}$ \\
\hline 4.10 & 2 & 2.0 & 50 & 16,583 & 829,150 & $2.4 \times 10^{-6}$ \\
\hline 5.2 & 5 & 2.2 & 45 & 06,991 & 314,595 & $1.6 \times 10^{-6}$ \\
\hline 5.3 & 1 & 1.8 & 56 & 12,505 & 700,280 & $1.4 \times 10^{-6}$ \\
\hline 5.10 & 8 & 1.7 & 59 & 10,740 & 633,660 & $1.3 \times 10^{-5}$ \\
\hline 5.13 & 1 & 8.2 & 12 & 16,570 & 198,840 & $5.0 \times 10^{-6}$ \\
\hline 7.3 & 1 & 1.5 & 67 & 16,528 & $1,107,376$ & $9.0 \times 10^{-7}$ \\
\hline 7.8 & 1 & 1.7 & 59 & 16,569 & 977,571 & $1.0 \times 10^{-6}$ \\
\hline 7.9 & 5 & 1.8 & 56 & 16,426 & 919,856 & $5.4 \times 10^{-6}$ \\
\hline 8.5 & 1 & 2.8 & 36 & 16,569 & 596,484 & $1.7 \times 10^{-6}$ \\
\hline $8.6^{*}$ & 1 & 9.0 & 11 & 16,239 & 178,629 & $5.6 \times 10^{-6}$ \\
\hline $8.6^{*}$ & 1 & 1.5 & 67 & 16,239 & $1,088,013$ & $9.2 \times 10^{-7}$ \\
\hline 8.10 & 1 & 4.6 & 22 & 16,533 & 363,726 & $2.7 \times 10^{-6}$ \\
\hline Average & 2 & 3.2 & 56 & - & - & $4.3 \times 10^{-6}$ \\
\hline
\end{tabular}

\# de novo mutations, number of de novo mutations detected in the oocyte. Average Het- $\%$ of de novo mut., the average heteroplasmy value for all de novo mutations found in the oocyte. Copy number from het- $\%$, the copy number the oocytes had when the mutation occurred using the formula: (1/average het$\%)^{*} 100$. *the heteroplasmy values of the variants in oocyte 8.6 differed markedly from each other and therefore the calculations were performed for both heteroplasmy values separately.

\section{Discussion}

The main aim of our study was to investigate the process of de novo mutagenesis in the mtDNA during oogenesis. Therefore, we analysed the mtDNA genome of 103 zebrafish oocytes by next-generation sequencing and compared it to somatic tissues of the corresponding mother. After applying statistical and biological filters, we characterized reliably 38 de novo mutations with heteroplasmy levels $\geq 1.5 \%$ in $18 \%$ of the oocytes. The de novo mutations were randomly distributed over the mtDNA genome, including some mutations causing a premature stop codon. 


\section{Robustness of identification de novo mutations with heteroplasmy} levels $\geq 1.5 \%$

We used the Nextera XT protocol to prepare libraries for sequencing on the HiSeq2000 platform, a system which has been successfully applied before for sequencing of the mitochondrial genome ${ }^{12 ;}{ }^{16}$. The identification of specific mutations with low heteroplasmy is limited by the noise level of the sequencing procedure ${ }^{14}$, which is around $1 \% 14 ; 16 ; 17$. As we cannot be sure noise levels will be equal between different runs and detection thresholds differ accordingly, we estimated the noise signal of our sequence run using a non-parametric, data-driven approach. Our approach is twofold. First, we estimated the median minimal coverage (Figure 4.1), which is a robust estimate of the entire mitochondrial genome, rendering a threshold coverage above which the calculated heteroplasmy value is reliable. Second, the noise level of the procedure is estimated for every position in the mtDNA based on the heteroplasmy levels reported for this position in all samples. By applying statistics, this allows identification of those variants for which the heteroplasmy level was significantly different from the background (or noise) signal ( $P$-value $\leq 0.01$ ), thereby excluding variants with a high occurrence in all samples, something which is not expected. In a previous report, power calculations were used to estimate the reliability of the reads $^{16}$. However, power calculations are theoretical and the use of such post-hoc calculations for the interpretation of available experimental results is debatable ${ }^{18}$. Another step in our analysis involves correction for the noise signal by subtracting the maternal heteroplasmy value from the detected heteroplasmy value. This correction is essential to know at which heteroplasmy value the de novo variant arose. A last step involved exclusion of variants that most likely occurred as a result of alignment artefacts. Based on our and others ${ }^{12 ; 16}$ experience with next generation sequencing, we excluded variants if they arose in close proximity to each other. Altogether, our data-driven statistical approach allows detection of de novo variants (heteroplasmy levels $\geq 1.5 \%$ ) with high reliability, which is corrected for potential differences in quality between sequence runs.

\section{De novo mutations are detected in oocytes with a low mtDNA copy number}

Our analysis pipeline allowed the detection of variants with a heteroplasmy value $\geq 1.5 \%$, which equals detection of a single mutated $\mathrm{mtDNA}$ molecule in a population of 65 or less. The estimated mtDNA copy number at which a de novo mutation occurred, ranged, based on the detected heteroplasmy levels of the mutations in the oocytes, from 11-67 (Table 4.3). In our analysis we only detected an mtDNA mutation in 18.9\% of the oocytes. The inheritance of the mtDNA through a bottleneck leads to low mtDNA levels in primordial germ cells (PGCs) at the bottom of the bottleneck ${ }^{6}$ and a mutation originating at this point may lead to higher heteroplasmy levels. In a previous study (Otten et al., in preparation), we have determined the mtDNA copy number in zebrafish PGCs isolated from several embryonic stages as on average $171 \mathrm{mtDNA}$ molecules at the bottom of the bottleneck, but with high variation in this number (StDev: 111). Based on these parameters we constructed a Gaussian distribution with mean 171 and standard deviation 111 . As $18.9 \%$ of the oocytes harboured a de 
novo mutation, this distribution allowed us to estimate that lower $18.9 \%$ (left tail of the distribution, z-score -0.88 ) of the PGCs possess $\leq 72.8$ mtDNA molecules. An mtDNA copy number of 72.8 correspond to a heteroplasmy level at mutation manifestation of $1.4 \%$, which is close to our detection limit of $1.5 \%$. This means our pipeline allows detection of de novo mutations in oocytes that were generated from germ cells with the lowest mtDNA content, which includes those germ cells most prone for acquiring a de novo mutation reaching a detectable heteroplasmy of $1.5 \%$ or higher. Together, this implies $\sim 20 \%$ of the oocytes had a bottleneck size at which de novo mutations could reach detectable heteroplasmy levels ( $\geq 1.5 \%)$.

During oogenesis in zebrafish, the mtDNA is replicated extensively. mtDNA replication is expected to occur almost exclusively by polymerase gamma (POLG) ${ }^{19}$; 20 , which is a two-subunit holoenzyme with high fidelity in nucleotide selection and incorporation, alongside with 3'-5' exonuclease proofreading functionality ${ }^{20}$. For all oocytes in which we detected a de novo mutation, the calculated average mutation rate was $4.3 \times 10^{-6}$ per nucleotide. This is in the same order of the reported POLG error frequencies, which range from $2 \times 10^{-6}$ to $10 \times 10^{-6}$ per nucleotide in different animals ${ }^{21 ; 22}$. Although POLG has a high accuracy, this suggests that errors made by POLG during the extensive replication during oogenesis are the main cause of de novo mutations in oocytes. Although replication errors are made continuously in all oocytes, only replication errors made when mtDNA copy numbers are low (e.g. 65 or lower) lead to detectable (heteroplasmy levels of $\geq 1.5 \%$ ) de novo mutations in mature oocytes, which was likely the case in 19 of the oocytes. Four of these oocytes had a higher mutation rate (range of $10^{-5}$ ). Although this can be a chance event, this could also be a reflection of individual differences in error rates of the mtDNA replicative machinery, or due to another mutagenic source being active in these oocytes. During OXPHOS, which is highly active during oogenesis ${ }^{23 ; 24}$, mutagenic ROS are being produced and this could be a factor contributing to differences in the mutation rate between oocytes. However, based on the mutation rates in most oocytes, errors made by POLG are most likely the dominant source of de novo mtDNA mutations in oocytes.

Our calculations on the mtDNA copy number and mutation rate are only applicable if random processes prevail and every mtDNA molecule is equally amplified. Due to genetic drift, leading to loss or fixation of mutations, especially in small sample sizes, and preferential selection, mutation loads can shift. This could explain the high mtDNA mutation load for one of the two de novo mutations detected in oocyte 8.6, although these mutations could also have manifested during separate replication cycles, as an extremely low mtDNA bottleneck size creates multiple cycles at which the mutation can manifest at heteroplasmy levels $\geq 1.5 \%$. Negative and positive selection has been demonstrated for specific some mutations ${ }^{25}$, further corroborating the possibility that non-random processes also influence the heteroplasmy level of de novo mtDNA mutations. In the case of selection, the physical and effective bottleneck sizes are different. The mtDNA molecules that actively replicate determine the effective bottleneck size, which can be lower than the physical bottleneck size when selective events result in only a subpopulation of mtDNA molecules being more actively replicated. The mtDNA copy number we have estimated here based on the heteroplasmy levels (11-67) correspond to effective mtDNA copy numbers, and might therefore be an underestimation of the physical bottleneck size. 


\section{De novo mutations in oocytes are potentially pathogenic}

The 38 de novo mutations with a frequency of $>1.5 \%$ were randomly distributed over the mtDNA genome. After correction for the size of the gene, the tRNA-Leu, tRNA-Trp and $t R N A-G l y$ genes had a high number of mutations per nucleotide. However, the numbers were too low to estimate this correctly [only one mutation in the tRNA-Leu and $t R N A-T r p$ genes were observed and two mutations in the tRNA-Gly gene] and mutations occurred in two oocytes from the same zebrafish. Furthermore, the tRNA genes as a group do not support a higher prevalence of mutations in the tRNA genes. The observed higher prevalence in the three tRNA genes is most likely due to the relatively small group of de novo mutations. On the contrary, a higher prevalence for mutations in the D-loop exists (Figure 4.2A). This is corroborated by the many variants observed in this mitochondrial control region ${ }^{26}$. Although preferences for the D-loop might exist from an evolutionary perspective, mutations in the mtDNA can arise anywhere in the mtDNA genome. In total, eight protein-coding genes were affected with little differences in the prevalence. 22 mutations were non-synonymous, including four mutations leading to a premature stop codon. No differences in prevalence in one of the three codon positions were found. This indicates the effect of a de novo mutation can be of any kind. The non-synonymous mutations, especially those causing a premature stop codon, are likely pathogenic, implying that also these severe mutations, which are rarely found in human patients, can occur de novo. Most likely in humans, these pathogenic mutations are filtered out by mitophagy ${ }^{27}$ or are at high levels not compatible with embryonic survival and remain at low levels unnoticed.

Given the high sequence homology ( $72 \%$; NCBI blast performed) between the mtDNA genome of zebrafish and humans and the high evolutionary conservation of the mtDNA bottleneck in animal species ${ }^{10 ; 14 ; 28 ; 29}$ our results indicate that the de novo risk might be similar among zebrafish and humans. Indeed, a study in 26 human oocytes ${ }^{30}, 7$ oocytes $(26.9 \%)$ were found to harbour de novo variants. This is close to the frequency of $18.9 \%$ we report her for zebrafish. This is further corroborated by a similar degree of variation in mtDNA copy number in human oocytes ${ }^{28}$, which suggest also variation in the mtDNA bottleneck size and subsequent differences in the de novo risk. In humans, it has been estimated that about $5 \%$ of the mutations in the mtDNA alter a conserved nucleotide and are thus potentially pathogenic ${ }^{30}$. As we found de novo mutations in $\sim 20 \%$ of the oocytes, this implies $\sim 1 \%$ of oocytes will carry a pathogenic de novo mutation [with a heteroplasmy level $\geq 1.5 \%$ ]. The presence of low-level mtDNA mutations in the oocyte could, after fertilization, lead to mtDNA disease later in life due to genetic drift, which could lead to fixation of the mutation ${ }^{31 ;}$ 32 or in the offspring of the following generation, as inheritance through the mtDNA bottleneck can cause shifts in the heteroplasmy level between mother and child, also leading to fixation of the mutant mtDNA ${ }^{33}$.

Despite the described similarities between zebrafish and humans, important reproductive and mtDNA differences should be taken into account. Zebrafish oocytes possess a much higher mtDNA content as human oocytes (factor $100^{29}$ ), mostly due to different implantation patterns. In humans, implantation occurs rapidly ${ }^{34}$, allowing a fast shift to the uterus for energy supply, while in zebrafish implantation is absent and energy must be supplied by the embryo itself. This lower mtDNA copy number in human oocytes might result in lower mtDNA numbers at the bottom of the bottleneck and the mtDNA genome might be even at higher risk for a de novo mutation to reach 
detectable heteroplasmy levels. This is supported by the high mutation frequency reported for the mammalian mtDNA compared to other animals, including fish ${ }^{35}$. In conclusion, our study in zebrafish has revealed that replication errors made during oogenesis are an important source of de novo mtDNA mutations and their location and heteroplasmy determine the eventual significance.

\section{Acknowledgements}

We thank Marie Winandy and Hélène Pendeville from the Zebrafish facility of Liège University for their assistance in collection of the zebrafish material. This work was supported by the Interreg IV program of the European council [the alma-in-silico project to M.M. and H.J.M.S.] and the E-RARE 2 project GENOMIT [R 50.02.12F to M.M.]. Part of this work has been made possible with the support of the Dutch Province of Limburg [M.A., M.G. and H.J.M.S.]

\section{References}

1. Lynch, M., Koskella, B., and Schaack, S. (2006). Mutation pressure and the evolution of organelle genomic architecture. Science 311, 1727-1730.

2. Barr, C.M., Neiman, M., and Taylor, D.R. (2005). Inheritance and recombination of mitochondrial genomes in plants, fungi and animals. The New phytologist 168, 39-50.

3. Brand, M.D. (2010). The sites and topology of mitochondrial superoxide production. Experimental gerontology 45, 466-472.

4. Taylor, R.W., and Turnbull, D.M. (2005). Mitochondrial DNA mutations in human disease. Nature reviews Genetics 6, 389-402.

5. Hellebrekers, D.M., Wolfe, R., Hendrickx, A.T., de Coo, I.F., de Die, C.E., Geraedts, J.P., Chinnery, P.F., and Smeets, H.J. (2012). PGD and heteroplasmic mitochondrial DNA point mutations: a systematic review estimating the chance of healthy offspring. Human reproduction update 18, 341-349.

6. Cree, L.M., Samuels, D.C., de Sousa Lopes, S.C., Rajasimha, H.K., Wonnapinij, P., Mann, J.R., Dahl, H.H., and Chinnery, P.F. (2008). A reduction of mitochondrial DNA molecules during embryogenesis explains the rapid segregation of genotypes. Nature genetics 40, 249 254.

7. Broughton, R.E., Milam, J.E., and Roe, B.A. (2001). The complete sequence of the zebrafish (Danio rerio) mitochondrial genome and evolutionary patterns in vertebrate mitochondrial DNA. Genome research 11, 1958-1967.

8. Howell, N., Halvorson, S., Kubacka, I., McCullough, D.A., Bindoff, L.A., and Turnbull, D.M. (1992). Mitochondrial gene segregation in mammals: is the bottleneck always narrow? Human genetics 90, 117-120.

9. Lee, H.S., Ma, H., Juanes, R.C., Tachibana, M., Sparman, M., Woodward, J., Ramsey, C., Xu, J., Kang, E.J., Amato, P., et al. (2012). Rapid mitochondrial DNA segregation in primate preimplantation embryos precedes somatic and germline bottleneck. Cell reports 1, 506-515.

10. Wolff, J.N., White, D.J., Woodhams, M., White, H.E., and Gemmell, N.J. (2011). The strength and timing of the mitochondrial bottleneck in salmon suggests a conserved mechanism in vertebrates. PloS one 6, e20522.

11. Kimmel, C.B., Ballard, W.W., Kimmel, S.R., Ullmann, B., and Schilling, T.F. (1995). Stages of embryonic development of the zebrafish. Developmental dynamics : an official publication of the American Association of Anatomists 203, 253-310.

12. McElhoe, J.A., Holland, M.M., Makova, K.D., Su, M.S., Paul, I.M., Baker, C.H., Faith, S.A., and Young, B. (2014). Development and assessment of an optimized next-generation DNA sequencing approach for the mtgenome using the Illumina MiSeq. Forensic science international Genetics 13, 20-29.

13. Li, H., and Durbin, R. (2010). Fast and accurate long-read alignment with Burrows-Wheeler transform. Bioinformatics 26, 589-595. 
14. Guo, Y., Li, C.I., Sheng, Q., Winther, J.F., Cai, Q., Boice, J.D., and Shyr, Y. (2013). Very low-level heteroplasmy mtDNA variations are inherited in humans. Journal of genetics and genomics = Yi chuan xue bao 40,607-615.

15. Li, H., and Durbin, R. (2009). Fast and accurate short read alignment with Burrows-Wheeler transform. Bioinformatics 25, 1754-1760.

16. Rebolledo-Jaramillo, B., Su, M.S., Stoler, N., McElhoe, J.A., Dickins, B., Blankenberg, D., Korneliussen, T.S., Chiaromonte, F., Nielsen, R., Holland, M.M., et al. (2014). Maternal age effect and severe germ-line bottleneck in the inheritance of human mitochondrial DNA. Proceedings of the National Academy of Sciences of the United States of America 111, 15474-15479.

17. Ma, J., Purcell, H., Showalter, L., and Aagaard, K.M. (2015). Mitochondrial DNA sequence variation is largely conserved at birth with rare de novo mutations in neonates. American journal of obstetrics and gynecology 212, 530 e531-538.

18. Hoenig, J.M., and Heisey, D.M. (2001). The Abuse of Power The Pervasive Fallacy of Power Calculations for Data Analysis Am Stat 55, 19-24.

19. Copeland, W.C., and Longley, M.J. (2003). DNA polymerase gamma in mitochondrial DNA replication and repair. TheScientificWorldJournal 3, 34-44.

20. Kaguni, L.S. (2004). DNA polymerase gamma, the mitochondrial replicase. Annual review of biochemistry 73, 293-320.

21. Kunkel, T.A., and Mosbaugh, D.W. (1989). Exonucleolytic proofreading by a mammalian DNA polymerase. Biochemistry 28, 988-995.

22. Longley, M.J., Nguyen, D., Kunkel, T.A., and Copeland, W.C. (2001). The fidelity of human DNA polymerase gamma with and without exonucleolytic proofreading and the p55 accessory subunit. The Journal of biological chemistry 276, 38555-38562.

23. Dumollard, R., Duchen, M., and Carroll, J. (2007). The role of mitochondrial function in the oocyte and embryo. Current topics in developmental biology 77, 21-49.

24. Van Blerkom, J., Davis, P.W., and Lee, J. (1995). ATP content of human oocytes and developmental potential and outcome after in-vitro fertilization and embryo transfer. Human reproduction $10,415-424$.

25. Steffann, J., Monnot, S., and Bonnefont, J.P. (2015). mtDNA mutations variously impact mtDNA maintenance throughout the human embryofetal development. Clin Genet 88, 416424.

26. Chinnery, P.F., Howell, N., Andrews, R.M., and Turnbull, D.M. (1999). Mitochondrial DNA analysis: polymorphisms and pathogenicity. Journal of medical genetics 36, 505-510.

27. Song, W.H., Ballard, J.W., Yi, Y.J., and Sutovsky, P. (2014). Regulation of mitochondrial genome inheritance by autophagy and ubiquitin-proteasome system: implications for health, fitness, and fertility. BioMed research international 2014, 981867.

28. Otten, A.B., and Smeets, H.J. (2015). Evolutionary defined role of the mitochondrial DNA in fertility, disease and ageing. Human reproduction update.

29. Otten, A.B., Theunissen, T.E., Lambrichs, E.H., Winandy, M., Tarbashevich, K., Raz, E., Vanoevelen, J.M., Van den Bosch, B.J., Muller, M., and Smeets, H.J. (2016). Differences in strength and timing of the mtDNA bottleneck between zebrafish germline and non-germline cells (chapter 3). Thesis, Auke Otten.

30. Jacobs, L., Gerards, M., Chinnery, P., Dumoulin, J., de Coo, I., Geraedts, J., and Smeets, $\mathrm{H}$. (2007). mtDNA point mutations are present at various levels of heteroplasmy in human oocytes. Molecular human reproduction 13, 149-154.

31. Greaves, L.C., Nooteboom, M., Elson, J.L., Tuppen, H.A., Taylor, G.A., Commane, D.M., Arasaradnam, R.P., Khrapko, K., Taylor, R.W., Kirkwood, T.B., et al. (2014). Clonal Expansion of Early to Mid-Life Mitochondrial DNA Point Mutations Drives Mitochondrial Dysfunction during Human Ageing. PLoS genetics 10, e1004620.

32. Yin, A.H., Peng, C.F., Zhao, X., Caughey, B.A., Yang, J.X., Liu, J., Huang, W.W., Liu, C., Luo, D.H., Liu, H.L., et al. (2015). Noninvasive detection of fetal subchromosomal abnormalities by semiconductor sequencing of maternal plasma DNA. Proceedings of the National Academy of Sciences of the United States of America.

33. Blok, R.B., Gook, D.A., Thorburn, D.R., and Dahl, H.H. (1997). Skewed segregation of the mtDNA nt 8993 (T-->G) mutation in human oocytes. American journal of human genetics 60 , 1495-1501.

34. Wimsatt, W.A. (1975). Some comparative aspects of implantation. Biology of reproduction 12, 1-40.

35. Lynch, M. (2006). The origins of eukaryotic gene structure. Molecular biology and evolution 23, 450-468. 



\title{
Chapter 5
}

\section{Mitochondrial DNA inheritance is}

\section{determined by genetic drift and negative}

\author{
and positive selection
}

Auke BC Otten*, Suzanne CEH Sallevelt*, Phillippa J Carling, Joseph CFM Dreesen, Marion Drüsedau, Sabine Spierts, Aimee DC Paulussen, Christine EM de Die-Smulders, Mary Herbert, David C Samuels, Hubert JM Smeets

* These authors contributed equally to this work 


\section{Summary}

Mitochondrial DNA (mtDNA) inherits maternally through a genetic bottleneck and genetic drift is considered the main driving mechanism. We determined mutation loads of three mtDNA mutations (m.3243A $>G, m .8993 T>G$ and $m .14487 T>C$ ) in preimplantation genetic diagnosis oocytes/zygotes/embryos. Genetic drift occurred, but non-random selection was clearly apparent, based on 1) correlation between $m .3243 A>G$ mutation load and estimated bottleneck size, 2) absence of m.3243A>G mutation loads $>80 \%$, and 3 ) skewed distribution for the m.8993T $>\mathrm{G}$ mutation. Together with data from a meta-analysis this implies that selection mechanisms can have a different effect on different mtDNA mutations. High m.3243A>G mutation loads are selected against, because the resulting complete OXPHOS deficiency prohibits oocyte growth and maturation (progression through meiosis). The OXPHOS deficiency of the m.8993T>G mutation is, even at high mutation loads, less severe. This mutation is positively selected for during oogenesis, because of an increased mitochondrial membrane potential (MMP), a hallmark of healthy mitochondria. The m.14487T $>C$ mutation is transmitted by random genetic drift only. We conclude that non-neutral selection mechanisms occur during mtDNA inheritance, which has implications for current treatment strategies.

\section{Introduction}

Mitochondrial diseases are a heterogeneous group of progressive multisystemic disorders, which can be caused by mutations in either the nuclear or the mitochondrial DNA (mtDNA). The mtDNA is a small, circular, double stranded, multi-copy genome. Over 150 confirmed pathogenic mutations have been reported in the mtDNA ${ }^{1}$, affecting about 1 in 5,000 individuals ${ }^{2}$. In most cases, these pathogenic mtDNA mutations are heteroplasmic, which is a co-existence of wild-type and mutated mtDNA genomes. The mtDNA bottleneck has evolved ${ }^{3}$ to prevent the germline from accumulating, low level heteroplasmic mutations. Based on observations in mice ${ }^{4 ; 5}$, the mtDNA bottleneck occurs as a result of a restriction in the number of mtDNA molecules during its maternal inheritance, followed by a strong amplification of these founder molecules. In carriers of heteroplasmic mtDNA mutations the bottleneck can have an adverse effect, leading to highly variable mutation loads in their oocytes and an unpredictable recurrence risk. The mechanisms underlying the mtDNA bottleneck are under debate, but to date, although it has been suggested that random genetic drift is predominant mechanism determining the distribution of mtDNA heteroplasmy ${ }^{6-8}$. When assuming random genetic drift, the segregation pattern of mtDNA mutations can be modelled mathematically by the Kimura distribution ${ }^{8}$. This model describes the probability distribution of heteroplasmy values in the offspring as a function of the amount of mutation carried over by the mother and the bottleneck size. The segregational unit is either the mtDNA molecule itself or a subset of mtDNA molecules that is preferentially amplified $^{9 ; 10}$. In mice the physical bottleneck size is estimated to be around 200 mtDNA copies per primordial germ cell (PGC) ${ }^{4 ; 7}$. The exact size of the bottleneck in humans is still under debate, just as the mechanisms underlying the shifts in mutation loads observed ${ }^{5}$. Some reports describe differences in segregation patterns between 
different mtDNA mutations ${ }^{11}$, which, apart from genetic drift, could also be caused by selection events ${ }^{12 ; 13}$ occurring during mtDNA transmission. When selection occurs, a physical and effective bottleneck can be discriminated. The mtDNA molecules that contribute to the mtDNA pool of the next generation determine the effective bottleneck size, which can be lower than the physical bottleneck size when only a subpopulation of mtDNA molecules is preferentially transmitted to the next generation. However, the picture is currently far from clear and the contribution of genetic drift and selection to mtDNA inheritance is still debated.

In the present study we investigated the underlying mechanisms of the mtDNA bottleneck by determining the mutation load of three mtDNA mutations ( $m .3243 A>G, m .8993 T>G$ and $m .14487 T>C$ ) in oocytes, zygotes and embryos from multiple preimplantation genetic diagnosis (PGD) cycles. We observed differences in segregation patterns among these mtDNA mutations, which could not be explained by genetic drift only and our data support the existence of mutation-specific selection factors during oogenesis. Besides the mechanisms already proposed in literature, we characterized two selective mechanisms that occurred during oogenesis. As these selective mechanisms alter the effective bottleneck size, these mechanisms give a plausible explanation for the mutation-specific genetic (effective) bottleneck size reported before ${ }^{14 ; 15}$. Our data was further supported by a meta-analysis of literature data on the segregation of $m .3243 A>G$ and $m .8993 T>G$ mutations. We find that germline selection depends on the degree that mutations affect the cellular OXPHOS-capacity of generating ATP or maintaining a healthy mitochondrial membrane potential (MMP). These findings are important for understanding mtDNA inheritance and will improve the prediction of the recurrence risk for carriers of mtDNA mutations considerably. This has also important implications for preimplantation genetic diagnosis (PGD) and mitochondrial replacement therapy, in which a fraction mutated mtDNA may be carried over during nuclear transplantation ${ }^{16}$. A mechanisms favouring selection, could result in amplification of the mutated genome during embryonic development.

\section{Material and Methods}

The study was performed with informed consent of the participants on rest material of routine clinical procedures, approved by the local ethical committee.

\section{Patients and PGD procedure}

Five female carriers (Table 5.1) of the m.3243A $>\mathrm{G}$ mutation, one carrier of the m.8993T $>G$ mutation and one carrier of the m.14487T $>C$ mutation were counselled for PGD. Verbal and written information on IVF and intracytoplasmic sperm injection (ICSI), single cell procedures, success rates of the procedures, risk of misdiagnosis, and the limited experience on the worldwide application of PGD for mitochondrial disorders was supplied to all carriers and their partners. Before the treatment, informed consent was given by both partners for both the procedure as well as for re-analysis of non-transferred and non-frozen oocytes and embryos. The IVF-ICSIPGD procedure has been performed as described before ${ }^{17}$. Briefly, blastomeres were biopsied at 3 days post-fertilization and washed three times in phosphate buffered 
saline solution supplemented with $1 \%$ polyvinylpyrrolidone molecular weight 360000 (Sigma-Aldrich Chemie, Zwijndrecht, the Netherlands) and $0.1 \mathrm{mg} / \mathrm{ml}$ phenol red (Sigma), and transferred to a $0.2 \mathrm{ml} \mathrm{PCR}$ reaction tube, followed by the mutation load analysis. Non-transferrable embryos and embryos not suited for cryopreservation were collected at day 4 , as described before ${ }^{17}$. Remaining fertilized oocytes, zygotes or single blastomeres collected during the procedure, as well as were washed three times in washing buffer, transferred to a $0.2 \mathrm{~mL} P C R$ reaction tube and stored at $-20^{\circ} \mathrm{C}$ until mutation load analysis. Furthermore, blood, hair, urine and/or muscle biopsies were taken from all carriers.

Table 5.1 Age and mutation loads in different tissues of carriers of the m.3243A>G, m.8993T>G and $\mathrm{m} .14487 \mathrm{~T}>\mathrm{C}$ mutation

\begin{tabular}{|l|c|c|c|c|c|}
\hline $\begin{array}{l}\text { m.3243A>G } \\
\text { carriers }\end{array}$ & Carrier \#1 & Carrier \#2 & Carrier \#3 & Carrier \#4 & Carrier \#5 \\
\hline $\begin{array}{l}\text { Age first PGD } \\
\text { cycle }\end{array}$ & 36 years & 30 years & 28 years & 32 years & 31 years \\
\hline Mutation-\% & $13 \%$ & $25 \%$ & $27 \%$ & $19 \%$ & $16 \%$ \\
$\begin{array}{l}\text { Blood } \\
\text { Hair }\end{array}$ & $26 \%$ & $10 \%$ & $29 \%$ & $26 \%$ & $29 \%$ \\
Urine & $55 \%$ & ND & $59 \%$ & $38 \%$ & $28 \%$ \\
Muscle & ND & $28 \%$ & ND & ND & ND \\
\hline Other carriers & m.8993T>G & m.14487T>C & & & \\
\hline $\begin{array}{l}\text { Age first PGD } \\
\text { cycle }\end{array}$ & 30 years & 40 years & & & \\
\hline $\begin{array}{l}\text { Mutation-\% } \\
\text { Blood }\end{array}$ & $4 \%$ & $34 \%$ & & & \\
Hair & $3 \%$ & ND & & & \\
Urine & $5 \%$ & $65 \%$ & & & \\
Muscle & ND & $55 \%$ & & & \\
\hline
\end{tabular}

\section{Mutation load analysis}

Primers used for PCR amplification conditions and digestion controls for the m.3243A $>\mathrm{G}$ and m.8993T $>\mathrm{G}$ mutations were as reported before ${ }^{17 ;} 18$, while for the $\mathrm{m} .14487 \mathrm{~T}>\mathrm{C}$ mutation, these were: ND6_199F \& ND6_199F (6-FAM) (ATACTCTTTCACCCACAGCAC), ND6_340R (ATAGTTTTTTTAATTTATTTAGGGCA), SpikeTspRI-F (vic, AGCTGGTAAAAGAGGCCTAAC) and SpikeTspRI-R (GCGCCGAATAATAGGTATAGTG) and digestion with TspRI. Samples were analysed by capillary electrophoresis on an ABI Prism 3730 Genetic Analyser followed by GeneScan analysis. To calculate the mutation load, the area of the mutation peak was divided by the sum of the peak area of the wild-type and mutation peak. The detailed statistical analysis for this procedure has been described before ${ }^{17}$.

For cleavage stage embryos from which multiple blastomeres were available, the average was calculated and reported as the mutation load for this embryo in order to exclude that a single embryo is repeatedly reported. In all cases, the mutation 
loads in individual blastomeres from a single embryo were not substantially different, consistent with previous findings ${ }^{17}$. Furthermore, mutations loads in blood, hair, urine and/or muscle of varies was established, using the same procedures (Table 5.1).

\section{Estimation of the effective bottleneck size}

We estimated the effective bottleneck size $\left(N_{\text {eff }}\right)$ for a carrier, assuming genetic drift, by using the equation $N_{\text {eff }}=-t / \ln (b)$, where $t$ is the number of mtDNA generations, which has been generally accepted to be 15 during embryogenesis ${ }^{8}$. As the average heteroplasmy level in the dataset $\left(p_{0}\right)$ is taken as an estimate of the amount of mutation carried over from the mother, this is used to determine the bottleneck parameter $(b)$ using equation 6 in reference ${ }^{8}$.

\section{Meta-analysis of published heteroplasmy data (m.3243A>G \& m.8993T>G)}

Blood heteroplasmy data was collected from literature for the m.3243A $>G(n=111)$ and the $m .8993 T>G(n=118)$ mutation (Table S5.1). The blood heteroplasmy levels for the m.3243A>G mutation were age-corrected to account for the loss of the exponential loss of mutated mtDNA in blood leukocytes by using an equation as described before 19. Furthermore, patients with mutation load(s) $<15 \%$ were excluded, as this improved the correlation between blood and muscle heteroplasmy (data not shown). For both mutations, the maternal heteroplasmy value was subtracted from the heteroplasmy value in the offspring (O-M). A Gaussian curve was fitted to the frequency histograms. To test whether the samples data from a Gaussian distribution with a mean equal hypothetical value of 0 (no selection bias) we used a one-sample $t$-test. Results were considered significant if the $P$-value was $<0.05$.

Table 5.2 | Average heteroplasmy levels and estimated bottleneck sizes for carriers with the $m .3243 A>G, m .8993 T>G$ and $m .14487 T>C$ mutation

\begin{tabular}{|c|c|c|c|}
\hline Carrier & $\mathbf{n}$ &  & $\begin{array}{c}\text { Effective bottleneck } \\
\text { size }\left(\mathrm{N}_{\text {off }}\right) \\
\text { (value }[95 \% \mathrm{Cl}])\end{array}$ \\
\hline$m .3242 A>G$ \#1 & 26 & $0.42 \pm 0.04$ & 83 [50-159] \\
\hline$m .3242 A>G$ \#2 & 16 & $0.34 \pm 0.05$ & 94 [50-233] \\
\hline$m .3242 A>G$ \#3 & 13 & $0.41 \pm 0.07$ & 49 [24-117] \\
\hline m.3242A>G \#4 & 26 & $0.33 \pm 0.04$ & 92 [55-173] \\
\hline $\mathrm{m} .3242 \mathrm{~A}>\mathrm{G} \# 5$ & 10 & $0.16 \pm 0.04$ & 152 [69-473] \\
\hline m.8993T>G & 46 & $0.05 \pm 0.03$ & $10[4-57]$ \\
\hline m.14487T $>C$ & 23 & $0.67 \pm 0.07$ & 21 [13-38] \\
\hline
\end{tabular}

n, number of unique offspring (oocytes, embryos or zygotes) for every carrier 


\section{Results and discussion}

\section{Individual bottleneck sizes under random genetic drift}

We analysed the mutation distribution in oocytes, zygotes and blastomeres of five carriers of the m.3243A $>\mathrm{G}$ mutation, one carrier of the m.8993T $>\mathrm{G}$ mutation and one carrier of the m.14487T>C mutation, for whom PGD was performed in our clinic (Figure 5.1). Based on these mutation loads, we calculated the effective (or mathematical) bottleneck size $\left(N_{\text {eff }}\right)$ for every carrier using the Kimura probability distribution $^{8}$ (Table 5.2). The Kimura distribution assumes random genetic drift only and gives an estimated bottleneck size value, which might be different from the actual mtDNA copy number value in the PGCs. The calculated $N_{\text {eff }}$ values are highly variable, being highest for the m.3243A $>G$ carriers, ranging, on average, from 49-152 mtDNA molecules, whereas $N_{\text {eff }}$ for the m.8993T $>G$ and the m.14487T $>C$ carriers are consistently smaller, 10 and 21 respectively. A clear difference (no overlap in confidence interval) exists between the m.8993T>G carrier and the m.3243A>G carrier with the lowest average heteroplasmy (carrier \#5), while there is only a slight overlap in confidence intervals between the m.8993T>G carrier and three of the $m .3243 A>G$ carriers. For the m.3243A $>G$ mutation, the reported values are close to the estimated effective bottleneck size of 108 in a female carrier of the mutation of which 82 single primary oocytes have been analysed ${ }^{8}$.

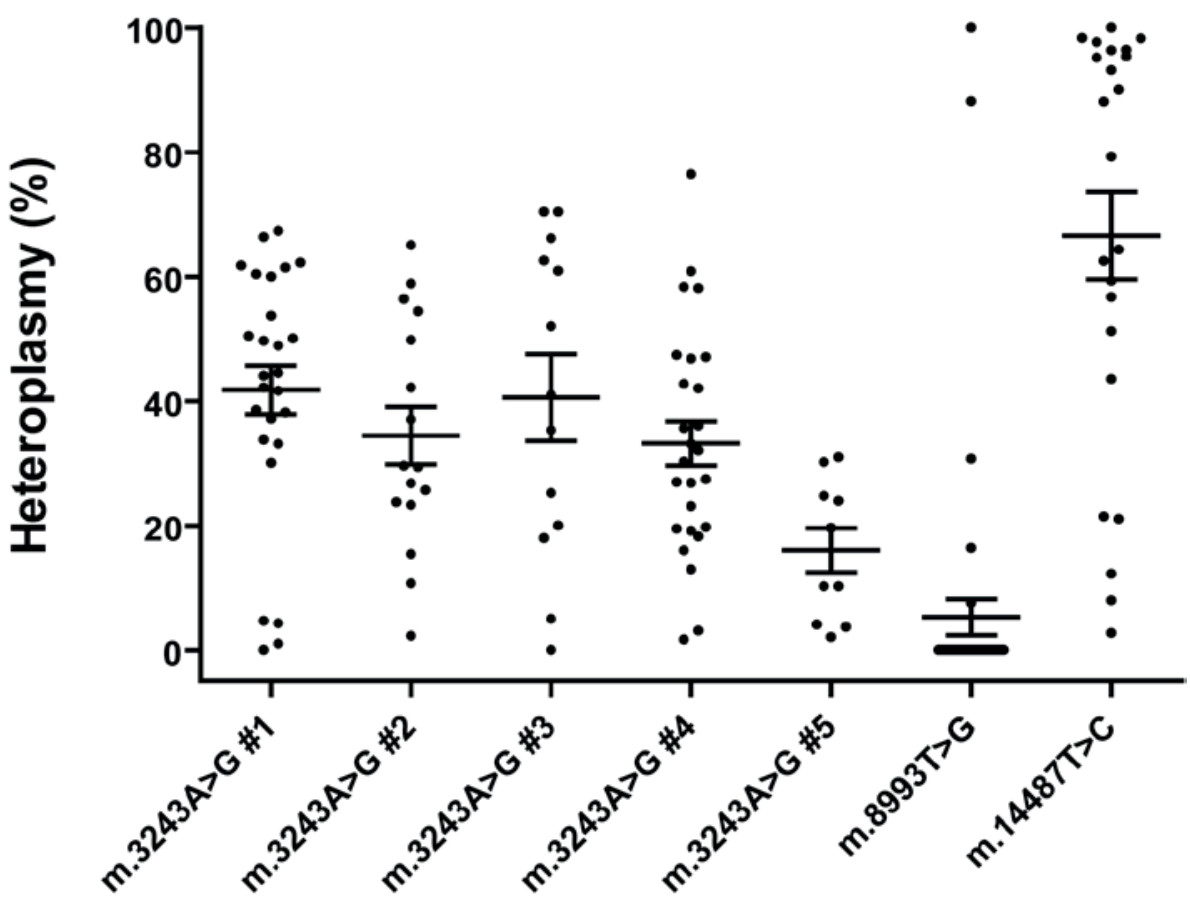

Figure 5.1 | Distribution of the percentages of $m .3243 A>G, m .8993 T>G$ and $m .14487 T>C$ mutation in surplus oocytes, blastomeres and zygotes from seven different carriers. When multiple blastomeres of a single cleavage stage embryo were analysed, the mutation load plotted for this embryo was the average of these blastomeres. Every dot thus represents the (average) mutation load of a single oocyte, embryo or zygote. Horizontal lines indicate average mutation loads with SEM. 


\section{Selection occurs during mtDNA inheritance}

The mtDNA segregation pattern can be explained by the Kimura neutral (genetic) drift theory when bottleneck sizes are variable, but the calculated, effective bottleneck sizes may not be equal to the actual physical bottleneck. It has recently been suggested that mtDNA mutations affect their segregation ${ }^{20}$, implying mechanisms other than random genetic drift to occur. If so, differences in the calculated effective bottleneck size, for which mutation-dependency has been reported ${ }^{15}$, are not caused by a lower physical bottleneck during germline transmission, but that mtDNA inheritance might be under the influence of mechanisms other than genetic drift.

Several observations in our data of the carriers indicate non-random mechanisms involved in mtDNA segregation. We observed a strong linear correlation $\left(P\right.$-value $=0.021, R^{2}=-0.93$, Figure 5.2$)$ between the effective bottleneck size and the average m.3243A>G mutation load in the offspring, which suggest that the effective bottleneck size decreases as the m.3243A $>\mathrm{G}$ mutation level in the offspring increases, implying it is not random. Maternal loads of $\sim 50 \%$ are expected, in case of random genetic drift, to yield the whole spectrum of mtDNA mutations in their offspring. Despite sufficient data points for $\mathrm{m} .3243 \mathrm{~A}>\mathrm{G}$ carrier \#1 and \#3 (mutations loads $\sim 50 \%$, Table 5.1 ), an absence of high mutation loads (>80\%, Figure 5.1 ) was noted, whereas we did observe eight samples in the offspring with a mutation load $<20 \%$. Although statistical normality tests are generally too tolerant to demonstrate subtle deviations (due to selection) from a Gaussian distribution, this absence of high mutation loads $(>80 \%)$ in offspring of $m .3243 A>G$ carriers is consistent with what has been described before ${ }^{6 ; 18}$, although in one case m.3243A>G mutation between 80 and $90 \%$ have been reported in one oocyte and one cleavage stage embryo ${ }^{21}$. Furthermore, it has been reported that carriers of the m.3243A>G mutation with blood heteroplasmy levels $>50 \%$ tended to have offspring with same or lower heteroplasmy, which was less pronounced for mothers with heteroplasmy $<50 \% 22$. Taken together, these data support a strong negative selection above an $m .3243 A>G$ mutation load of $80-90 \%$. At lower levels, as demonstrated by a meta-analysis of literature data on differences in blood heteroplasmy levels between mothers and offspring, the role of selection is marginal, as differences in blood heteroplasmy for the m.3243A $>\mathrm{G}$ mutation between mothers and offspring (O-M) equals zero (Figure 5.3, $P$-value $=$ 0.20).

The distribution of mutation loads in oocytes, zygotes and cleavage embryos of the m.8993T>G carrier was fundamentally different from the m.3243A>G mutation and clearly non-Gaussian, showing a non-random, skewed segregation for this mutation, also indicating selective events are active in during m.8993T>G transmission. Although results for this mutation are limited to one carrier, comparable results have been reported in different studies:

- Seven oocytes from a female m.8993T>G carrier were tested and six oocytes had a mutant load of $>95 \%$, while the other had no signs of the mutation ${ }^{23}$.

- $\quad$ PGD for an asymptomatic carrier of the m.8993T>G mutation resulted in one embryo homoplasmic for the mutation and two embryos that were mutationfree ${ }^{24}$.

- A large collection of pedigrees with a mutation at nucleotide 8993 (which can be either $T>G$ or $T>C^{25}$ ) showed that the mutation load distribution was 
skewed to the extremes. The proportion of children with a high mutation load is increased, when the mutant load in the mother is high as well. This is in particular the case for the m.8993T>G mutation ${ }^{26}$.

- Prenatal analysis for the $m .8993 T>G$ mutation in six pregnancies indicated three times a mutation load $>95 \%$, two times undetectable mutation loads $(0 \%)$ and one had intermediate $(30-80 \%)$ mutation loads ${ }^{27}$. Intermediate mutation loads (30-40\%) in three embryos after a PGD procedure have been reported only once ${ }^{28}$. However, the remaining seven embryos analysed during this PGD procedure had either a very low (one embryo with $2.5 \%$ ) or a very high (six embryos $>95 \%$ ) mutation load, which supports the predominantly skewed segregation of the $m .8993 T>G$ mutation ${ }^{28}$.

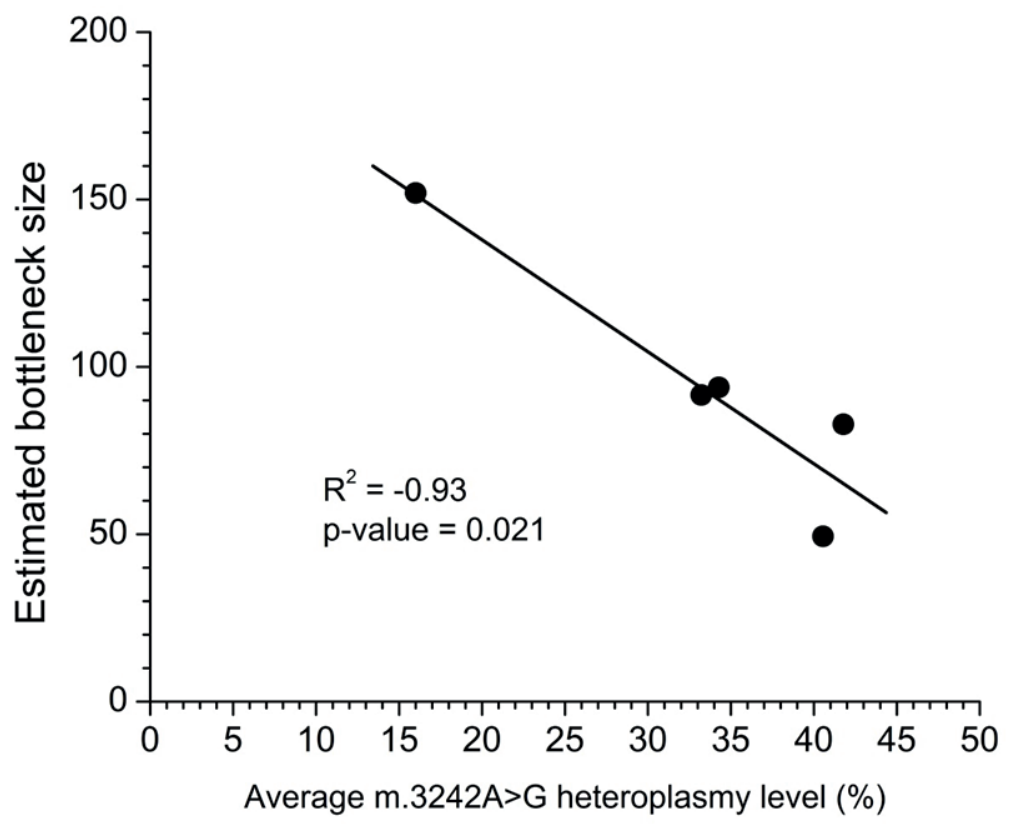

Figure 5.2 | Correlation between the average heteroplasmy value in offspring samples (oocytes, zygotes and blastomeres) from the five m.3243A $>G$ carriers and the estimated bottleneck size based on the Kimura distribution. After correlation analysis (Pearson), there was a significant correlation ( $P$-value $0.021)$, indicating that the bottleneck size decreases as the m.3243A>G mutation load increases $\left(R^{2}=\right.$ $-0.93)$.

We obtained additional evidence for a non-random, skewed segregation of the m.8993T $>\mathrm{G}$ mutation by performing a meta-analysis (Figure 5.3) of differences in blood heteroplasmy levels between mothers and offspring (O-M). The m.8993T>G mutation had a distribution that was significantly different from zero $(P$-value $<0.0001)$. In these family studies an ascertainment bias could have occurred, because the families were identified through an affected child with a very high mutation load. The impact of such a bias was reduced by ascertainment through the mother, without the proband, which is also the case in offspring analysis as performed in the families we used for meta-analysis, our carriers counselled for PGD, other reported PGD analysis ${ }^{24 ;}$, and prenatal analysis ${ }^{27}$. Taken together, these data indicate the segregation of the $m .8993 T>G$ mutation is under the influence of non-random mechanisms. 
For the m.14487T>C mutation limited information is available in literature and only one carrier was assessed in our study. Unlike the distribution in the m.3243A>G and the m.8993T>G mutation, practically all mutation loads are represented and the distribution appears Gaussian, although due to an overrepresentation of samples with mutation loads $>90 \%$, selection mechanisms cannot be excluded. However, selective events are expected to have a more minor role on inheritance of the m.14487T >C mutation, as all mutation loads were tolerated, but on a single carrier, we cannot draw firm conclusions. Obviously, data regarding segregation patterns and bottleneck sizes for mutations in human embryos is limitedly available, but the mtDNA mutation specific mutant load distribution profiles described here are in line with other observations ${ }^{20}$. As these patterns are most likely result from selection events, we investigated the underlying mechanisms.
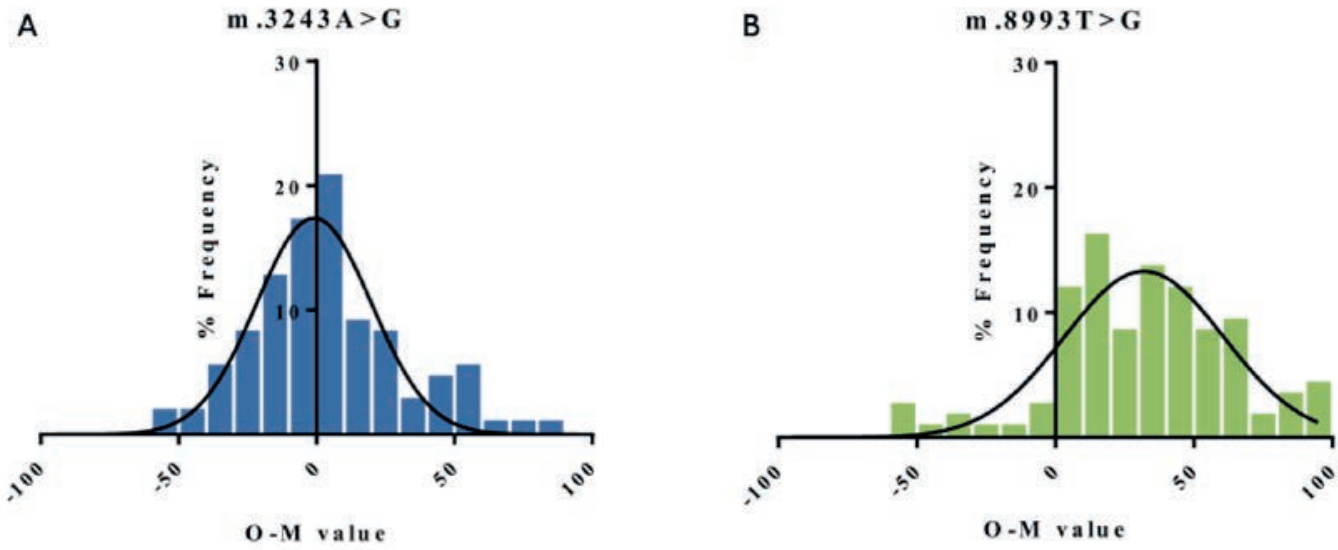

Figure 5.3 | Frequency histograms of O-M values for m.3243A>G and m.8993T>G transmissions. Heteroplasmy data from blood for mother to child transmissions were compiled from published studies of the m.3243A>G $(n=111)$ and the m.8993T>G mutation $(n=118)$. The blood heteroplasmy for the m.3243A>G mutation was corrected for age to avoid potential bias. O-M values represent the shift in heteroplasmy between generations ( $O=$ offspring, $M=$ mother) of $A$ ) $m .3243 A>G$ and $B$ ) $m .8993 T>G$ Frequency. Histograms were plotted for both mutations, and a Gaussian curve fitted to each plot.

\section{Selection on ATP production capacity of cellular mitochondria}

Primordial germ cells are highly dependent on glycolysis ${ }^{29}$, while developing oocytes rely on OXPHOS for ATP production ${ }^{30 ; 31}$ in order to establish a minimal cytoplasmic steady-state ATP level in mature oocytes ${ }^{31 ; 32}$ and (activation of) early embryonic development $^{33}$ (Figure 5.4). The OXPHOS dependency during oogenesis is corroborated by the finding that disturbed mitochondrial metabolism leads to a drop in ATP levels that interferes with oocyte maturation and preimplantation embryogenesis ${ }^{34}$. The ATP demand can only be satisfied by a highly active mitochondrial OXPHOS ${ }^{33}$, as the glycolysis pathway is not active ${ }^{35}$.

The m.3243A>G mutation affects the mitochondrial tRNA responsible for leucine-UUR ${ }^{36}$ resulting in defective assembly of complexes I, IV and V in myoblasts homoplasmic for m.3243A $>G^{37}$ and, subsequently, in a decrease in the ATP generating capacity. As we did not observe any oocytes, zygotes or blastomeres with $>80 \%$ 
m.3243A>G mutation load, this is most likely a threshold where the large ATP demands during oogenesis cannot be met anymore despite the high number of mitochondria and mtDNA (Figure 5.4), and are selected against by a failure of oocyte maturation. The figure of $80 \%$ is in line with what has been reported for cybrids harbouring the m.3243A>G mutation ${ }^{38 ;} 39$, where oxidative capacity started to decline in cybrids with $60 \%$ mutation load or higher and was completely lost (maximal respiration <10\% compared to low mutation loads) at $90 \%$ mutation load, due to an almost complete lack of complex I, and severely reduced abundance of complex III and IV. ATP levels rapidly declined in cybrids with $>80 \%$ mutation load with an upregulation of the glycolysis pathway ${ }^{39}$. This loss in capacity of the mitochondrial energy-generating system has also been observed in muscle biopsies with high m.3243A>G mutation loads $^{40}$. An adaptive mechanisms has been suggested enabling mutant embryos to escape selection by activating mtDNA replication ${ }^{20}$. The net result would be a large availability of both mutant and wild-type mtDNA molecules, the latter being able to rescue the energy deficiency induced by the m.3243A $>\mathrm{G}$ mutation. This would also explain that very high ( $90 \%)$ m.3243A>G mutations have been reported ${ }^{21}$, but $100 \%$ mutation load s were never observed.

The m.8993T>G mutation affects the ATPase 6 subunit ${ }^{41}$, and as such impairs the assembly and stability of the terminal complex (complex $V$ ) of the OXPHOS machinery ${ }^{42}$, during which ATP is generated out of a proton gradient. As we observed very high (>95\%) mutation load in oocytes, zygotes and cleavage embryos of carriers, we concluded that negative selection on ATP production capacity did not occur during oogenesis. Indeed, a study in lymphocytes showed that despite high m.8993T>G mutation loads (80-95\%), still 25-35\% capacity in ATP synthesis remained ${ }^{43 ;} 44$. Likewise, cybrids homoplasmic for the m.8993T>G mutation still displayed $>25 \%$ residual ATP synthesis ${ }^{45}$. This implies that, even when homoplasmic for the m.8993T>G mutation, oxidative respiration is preserved to a moderate degree, which, in combination with a high abundance of mitochondria in the oocyte (between 19,000 and $25,000^{34}$ ), appears sufficient to produce cytoplasmic ATP for oogenesis to proceed normally.

The m.14487T>C mutation is present in the ND6 subunit of complex $\mathrm{I}^{46}$, thereby affecting its stability and assembly ${ }^{47}$. The random distribution argues against negative selection and, indeed, biochemical investigations showed that in skeletal muscle, complex I activity was only modestly decreased ${ }^{48}$, precluding negative selection on ATP production capacity ${ }^{49}$. Selection on ATP production capacity has also been observed in the germline of a mouse model for mtDNA disease ${ }^{50}$. A severe ND6 mutation, causing a premature termination of the protein and a subsequent inactivation of OXPHOS, was selectively eliminated during oogenesis within four generations, while a milder mutation in CO1, causing only $50 \%$ decrease in OXPHOS, was retained throughout multiple generations. In conclusion, cellular germline selection against mtDNA mutations depends on the degree that mutations affect the cellular OXPHOScapacity of generating ATP in combination with the mtDNA copy number.

\section{Selection on membrane potential of mitochondria}

In contrast to the m.3243A $>\mathrm{G}$ and m.14487T>C mutations, oocytes, zygotes and embryos of the m.8993T>G mutation carriers demonstrated skewing to the extremes, 
which suggests that if the mutation is transmitted above a certain level, it gets rapidly fixed at a high mutation load, indicating positive selection for the m.8993T >G mutation. This can be explained by changes in the mitochondrial membrane potential (MMP). In the metabolically active mitochondria of the oocytes, complex $\mathrm{V}$ is the major regulator of the MMP, which must be maintained to support ATP synthesis ${ }^{33}$. As a result, an increased MMP is necessary for oocyte maturation and for the competence of an embryo to develop (Figure 5.4) 34;51. Consequently, MMP has developed as a sensor of normal, healthy OXPHOS activity, which is being selected for during oogenesis at the level of the Balbiani bodies, which contain aggregates of highly active mitochondria with a high MMP52-54. These Balbiani bodies are preferentially transmitted to the $P G C s^{55}$. It has been reported that the m.8993T>G mutation causes an inefficient coupling between proton transport and ATP production ${ }^{44}$. In lymphocytes with $>75 \%$ m.8993T > G mutation load or with a blocked complex $\mathrm{V}^{43}$, the MMP was significantly increased compared to wild-type mitochondria, because the proton gradient could not be consumed by complex V. Since mitochondrial with high m.8993T >G mutation loads possess a higher MMP, these mitochondria are more likely to be recruited in Balbiani bodies and subsequently transmitted to the offspring, eventually leading to (almost) homoplasmy. This most likely occurs above a certain mutation load, when the selection advantage overrides the genetic drift. Below this threshold, the m.8993T $>\mathrm{G}$ mutation will go to $0 \%$ or rarely generate low-moderate m.8993T>G mutation loads ${ }^{28}$ during the 15 germ cell divisions that occur during early embryogenesis ${ }^{8}$. Together, positive selection events and genetic drift provide for the first time, a plausible explanation for the consistent skewed segregation of the m.8993T>G mutation. By contrast, for the m.8993T>C mutation, which is caused by different substitution of the same nucleotide, a non-skewed, more random segregation pattern has been described, which can be explained by the fact that ATP production is relatively preserved and MMP is not significantly affected by the $T>G$ substitution ${ }^{43}$.

In case of the m.3243A>G mutation, the almost complete loss of OXPHOS at high mutation loads causes the MMP to drop, while mitochondria with mainly wildtype mtDNA molecules (with a higher MMP) would therefore have a preference to be recruited in Balbiani bodies, adding up to the absence of high m.3243A $>\mathrm{G}$ mutation loads. Mitochondria with lower mutation loads have a similar MMP compared to those without the mutation and have an equal chance to be recruited in the Balbiani body. This is also the case for the m.14487T $>\mathrm{C}$ mutation, which affects the OXPHOS system only modestly and has no effect on the MMP. Although convincing evidence exists for a role of the MMP in selecting mitochondria during oogenesis, definite proof would come from MMP measurements in oocytes harbouring mtDNA point mutations. 
Table 5.3 | Relative contribution of genetic drift and selection contribute to the segregation of three mtDNA mutations.

\begin{tabular}{|c|c|c|c|}
\hline Segregational mechanism & m.3243A>G & $\mathrm{m} .8993 \mathrm{~T}>\mathrm{G}$ & m.14487T >C \\
\hline Genetic drift? & + & + & + \\
\hline $\begin{array}{l}\text { Selection on OXPHOS } \\
\text { function? }\end{array}$ & NEGATIVE & POSITIVE & NEUTRAL \\
\hline $\begin{array}{l}\text { - OXPHOS assembly } \\
\text { affected? }\end{array}$ & $\begin{array}{l}\text { At } 90-100 \% \text { OX- } \\
\text { PHOS assembly } \\
\text { lost }(\mathrm{Cl})\end{array}$ & Only CV affected & $\begin{array}{l}\text { Stability and } \\
\text { assembly Cl } \\
\text { affected }\end{array}$ \\
\hline $\begin{array}{l}\text { - ATP generating } \\
\text { capacity affected? }\end{array}$ & $\begin{array}{l}>80 \% \text { respiratory } \\
\text { defect ATP drops }\end{array}$ & $\begin{array}{l}100 \% \text { : residual } \\
\text { activity } 20-30 \%\end{array}$ & $\begin{array}{l}\text { OXPHOS } \\
\text { capacity } \\
\text { preserved }\end{array}$ \\
\hline $\begin{array}{l}\text { - Membrane potential } \\
\text { ('selection sensor') } \\
\text { affected }\end{array}$ & Severely reduced & $\begin{array}{l}\text { Higher due to in- } \\
\text { efficient coupling }\end{array}$ & No effect \\
\hline
\end{tabular}

Cl, complex I. CV, complex V.

\section{Conclusion}

Random genetic drift is an important mechanism involved in the segregation of mtDNA. The effects of genetic drift are amplified by the drastic reduction in the mtDNA molecules transmitted to the offspring by the bottleneck mechanism. Based on the mutation distribution in oocytes, zygotes and embryonic cells, a considerable variation in the bottleneck sizes (10 to 152 copies) between carriers and mutations is calculated, causing different rates of genetic drift. However, non-random mutationspecific segregation patterns indicate that other mechanisms are active as well and that genetic drift is supplemented with mutation-specific negative (cellular) and positive (organellar) selection mechanisms, based on optimal OXPHOS capacity and MMP. Future studies are needed to test whether the selective mechanisms we propose here (Table 5.3) are able to explain the segregation of all mtDNA mutations.

Defining the underlying mechanisms of the mtDNA bottleneck are not only important to predict the recurrence risk for mtDNA mutations carriers more accurately, it also has considerable implications for the threshold levels for embryo replacement during PGD, which are currently defined solely on the genetic drift model. Positive selection of a mutation does affect the threshold, below which embryos are being transferred safely, as so far only genetic drift is considered. For mutations under positive selection, the threshold should be reduced. Furthermore, it can have consequences for mitochondrial replacement therapy, a procedure during which the nuclear genome from an mtDNA mutation-harbouring zygote of oocytes is transferred to an enucleated acceptor, harbouring mutation-free $\mathrm{mtDNA}^{56}$. During this procedure some carry-over of mtDNA can occur, leading to heteroplasmy, which even in the case of neutral variants can be preferentially replicated during ESC propagation ${ }^{57}$. It 
is likely that this effect would be amplified in the case of mutations that confer positive selection. It will therefore be particularly important to transfer the lowest possible levels of mtDNA in the event that mitochondrial replacement techniques are used to prevent transmission of such mutations. The combination of genetic drift and (positive) selection leaves a chance that those mtDNA mutations (like the m.8993T>G) can reach higher levels than anticipated by carry-over only. For PGD and mitochondrial replacement therapy this could be taken into account.

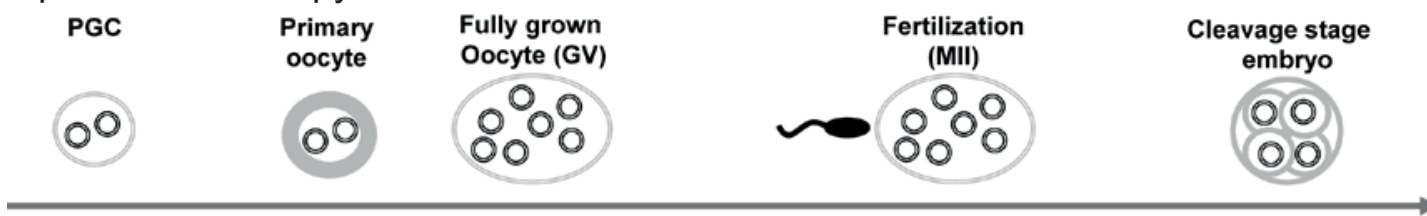

\section{Metabolic dependency}

Glycolysis OXPHOS

Mitochondrial membrane potential (MMP)

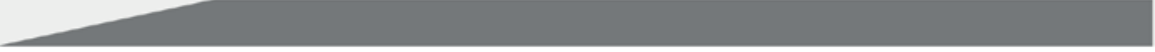

Total mtDNA content

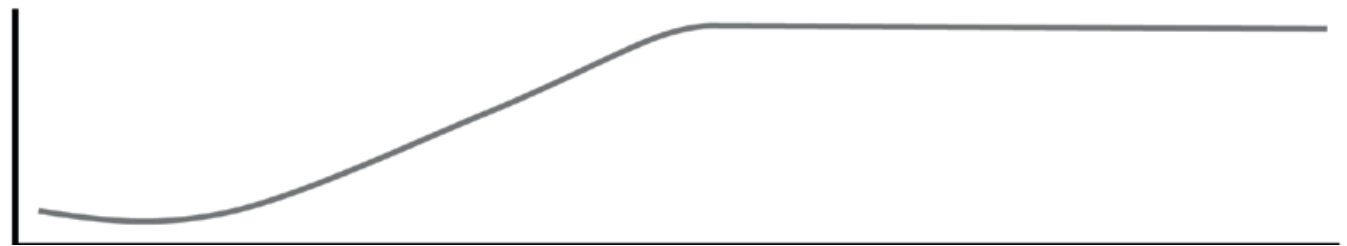

Figure 5.4 | Overview of metabolic dependency, mitochondrial membrane potential and mtDNA content during oogenesis and early embryogenesis. The primordial germ cells (PGC) are highly dependent on glycolysis. During oogenesis, the glycolysis becomes blocked and ATP is exclusively produced by mitochondrial OXPHOS, which becomes essential for oocyte maturation and the activation of early embryonic development. Likewise, a high mitochondrial membrane potential (MMP) is essential for oocyte maturation, indicating OXPHOS-dependent ATP production. Furthermore, mtDNA content increases during oogenesis, allowing compensation in OXPHOS defects, as well as selection for highly active mitochondria with high MMP.

\section{Acknowledgements}

We are grateful to GROW-School of Oncology and Developmental Biology for their fellowship to A.B.C.O. and H.J.M.S.

\section{References}

1. Hellebrekers, D.M., Wolfe, R., Hendrickx, A.T., de Coo, I.F., de Die, C.E., Geraedts, J.P., Chinnery, P.F., and Smeets, H.J. (2012). PGD and heteroplasmic mitochondrial DNA point mutations: a systematic review estimating the chance of healthy offspring. Human reproduction update 18, 341-349. 
2. Gorman, G.S., Schaefer, A.M., Ng, Y., Gomez, N., Blakely, E.L., Alston, C.L., Feeney, C., Horvath, R., Yu-Wai-Man, P., Chinnery, P.F., et al. (2015). Prevalence of nuclear and mitochondrial DNA mutations related to adult mitochondrial disease. Annals of neurology 77, 753-759.

3. Otten, A.B., and Smeets, H.J. (2015). Evolutionary defined role of the mitochondrial DNA in fertility, disease and ageing. Human reproduction update.

4. Cree, L.M., Samuels, D.C., de Sousa Lopes, S.C., Rajasimha, H.K., Wonnapinij, P., Mann, J.R., Dahl, H.H., and Chinnery, P.F. (2008). A reduction of mitochondrial DNA molecules during embryogenesis explains the rapid segregation of genotypes. Nature genetics 40, 249-254.

5. Hauswirth, W.W., and Laipis, P.J. (1982). Mitochondrial DNA polymorphism in a maternal lineage of Holstein cows. Proceedings of the National Academy of Sciences of the United States of America 79, 4686-4690.

6. Brown, D.T., Samuels, D.C., Michael, E.M., Turnbull, D.M., and Chinnery, P.F. (2001). Random genetic drift determines the level of mutant mtDNA in human primary oocytes. American journal of human genetics $68,533-536$.

7. Jenuth, J.P., Peterson, A.C., Fu, K., and Shoubridge, E.A. (1996). Random genetic drift in the female germline explains the rapid segregation of mammalian mitochondrial DNA. Nature genetics 14, 146-151.

8. Wonnapinij, P., Chinnery, P.F., and Samuels, D.C. (2008). The distribution of mitochondrial DNA heteroplasmy due to random genetic drift. American journal of human genetics 83, 582-593.

9. Cao, L., Shitara, H., Horii, T., Nagao, Y., Imai, H., Abe, K., Hara, T., Hayashi, J., and Yonekawa, H. (2007). The mitochondrial bottleneck occurs without reduction of mtDNA content in female mouse germ cells. Nature genetics 39, 386-390.

10. Wai, T., Teoli, D., and Shoubridge, E.A. (2008). The mitochondrial DNA genetic bottleneck results from replication of a subpopulation of genomes. Nature genetics 40, 1484-1488.

11. Howell, N., Halvorson, S., Kubacka, I., McCullough, D.A., Bindoff, L.A., and Turnbull, D.M. (1992). Mitochondrial gene segregation in mammals: is the bottleneck always narrow? Human genetics 90, 117-120.

12. Meiklejohn, C.D., Montooth, K.L., and Rand, D.M. (2007). Positive and negative selection on the mitochondrial genome. Trends in genetics : TIG 23, 259-263.

13. Stewart, J.B., Freyer, C., Elson, J.L., Wredenberg, A., Cansu, Z., Trifunovic, A., and Larsson, N.G. (2008). Strong purifying selection in transmission of mammalian mitochondrial DNA. PLoS biology 6 , e10.

14. Li, M., Rothwell, R., Vermaat, M., Wachsmuth, M., Schroder, R., Laros, J.F., van Oven, M., de Bakker, P.I., Bovenberg, J.A., van Duijn, C.M., et al. (2016). Transmission of human mtDNA heteroplasmy in the Genome of the Netherlands families: support for a variable-size bottleneck. Genome research.

15. Wilson, I.J., Carling, P.J., Alston, C.L., Floros, V.I., Pyle, A., Hudson, G., Sallevelt, S.C., Lamperti, C., Carelli, V., Bindoff, L.A., et al. (2016). Mitochondrial DNA sequence characteristics modulate the size of the genetic bottleneck. Human molecular genetics 25, 1031-1041.

16. Mitalipov, S., and Wolf, D.P. (2014). Clinical and ethical implications of mitochondrial gene transfer. Trends Endocrinol Metab 25, 5-7.

17. Sallevelt, S.C., Dreesen, J.C., Drusedau, M., Spierts, S., Coonen, E., van Tienen, F.H., van Golde, R.J., de Coo, I.F., Geraedts, J.P., de Die-Smulders, C.E., et al. (2013). Preimplantation genetic diagnosis in mitochondrial DNA disorders: challenge and success. Journal of medical genetics 50, 125-132.

18. Monnot, S., Gigarel, N., Samuels, D.C., Burlet, P., Hesters, L., Frydman, N., Frydman, R., Kerbrat, V., Funalot, B., Martinovic, J., et al. (2011). Segregation of mtDNA throughout human embryofetal development: m.3243A $>\mathrm{G}$ as a model system. Human mutation $32,116-125$.

19. Rajasimha, H.K., Chinnery, P.F., and Samuels, D.C. (2008). Selection against pathogenic mtDNA mutations in a stem cell population leads to the loss of the $3243 A-->G$ mutation in blood. American journal of human genetics 82, 333-343.

20. Steffann, J., Monnot, S., and Bonnefont, J.P. (2015). mtDNA mutations variously impact mtDNA maintenance throughout the human embryofetal development. Clin Genet 88, 416-424.

21. Treff, N.R., Campos, J., Tao, X., Levy, B., Ferry, K.M., and Scott, R.T., Jr. (2012). Blastocyst preimplantation genetic diagnosis (PGD) of a mitochondrial DNA disorder. Fertility and sterility 98, 1236-1240.

22. Uusimaa, J., Moilanen, J.S., Vainionpaa, L., Tapanainen, P., Lindholm, P., Nuutinen, M., Lopponen, T., Maki-Torkko, E., Rantala, H., and Majamaa, K. (2007). Prevalence, segregation, and phenotype of the mitochondrial DNA $3243 \mathrm{~A}>\mathrm{G}$ mutation in children. Annals of neurology 62, 278-287.

23. Blok, R.B., Gook, D.A., Thorburn, D.R., and Dahl, H.H. (1997). Skewed segregation of the 
mtDNA nt 8993 (T-->G) mutation in human oocytes. American journal of human genetics 60, 1495-1501.

24. Steffann, J., Frydman, N., Gigarel, N., Burlet, P., Ray, P.F., Fanchin, R., Feyereisen, E., Kerbrat, V., Tachdjian, G., Bonnefont, J.P., et al. (2006). Analysis of mtDNA variant segregation during early human embryonic development: a tool for successful NARP preimplantation diagnosis. Journal of medical genetics 43, 244-247.

25. White, S.L., Collins, V.R., Wolfe, R., Cleary, M.A., Shanske, S., DiMauro, S., Dahl, H.H., and Thorburn, D.R. (1999). Genetic counseling and prenatal diagnosis for the mitochondrial DNA mutations at nucleotide 8993. American journal of human genetics 65, 474-482.

26. Dahl, H.H., Thorburn, D.R., and White, S.L. (2000). Towards reliable prenatal diagnosis of mtDNA point mutations: studies of nt8993 mutations in oocytes, fetal tissues, children and adults. Human reproduction 15 Suppl 2, 246-255.

27. White, S.L., Shanske, S., Biros, I., Warwick, L., Dahl, H.M., Thorburn, D.R., and Di Mauro, S. (1999). Two cases of prenatal analysis for the pathogenic T to $G$ substitution at nucleotide 8993 in mitochondrial DNA. Prenatal diagnosis 19, 1165-1168.

28. Thorburn, D.R., Wilton, L., and Stock-Myer, S. (2009). Healthy baby girl born following PGD for the mitochondrial DNA mutation m.8993T>G. Mitochondrion 10, 222.

29. Ramalho-Santos, J., Varum, S., Amaral, S., Mota, P.C., Sousa, A.P., and Amaral, A. (2009). Mitochondrial functionality in reproduction: from gonads and gametes to embryos and embryonic stem cells. Human reproduction update $15,553-572$.

30. Dumollard, R., Duchen, M., and Carroll, J. (2007). The role of mitochondrial function in the oocyte and embryo. Current topics in developmental biology 77, 21-49.

31. Van Blerkom, J., Davis, P.W., and Lee, J. (1995). ATP content of human oocytes and developmental potential and outcome after in-vitro fertilization and embryo transfer. Human reproduction 10, 415-424.

32. Zeng, H.T., Yeung, W.S., Cheung, M.P., Ho, P.C., Lee, C.K., Zhuang, G.L., Liang, X.Y., and O, W.S. (2009). In vitro-matured rat oocytes have low mitochondrial deoxyribonucleic acid and adenosine triphosphate contents and have abnormal mitochondrial redistribution. Fertility and sterility 91, 900-907.

33. Dumollard, R., Marangos, P., Fitzharris, G., Swann, K., Duchen, M., and Carroll, J. (2004). Sperm-triggered $[\mathrm{Ca} 2+]$ oscillations and $\mathrm{Ca} 2+$ homeostasis in the mouse egg have an absolute requirement for mitochondrial ATP production. Development 131, 3057-3067.

34. Van Blerkom, J. (2011). Mitochondrial function in the human oocyte and embryo and their role in developmental competence. Mitochondrion 11, 797-813.

35. Shyh-Chang, N., Daley, G.Q., and Cantley, L.C. (2013). Stem cell metabolism in tissue development and aging. Development 140, 2535-2547.

36. Goto, Y., Nonaka, I., and Horai, S. (1990). A mutation in the tRNA(Leu)(UUR) gene associated with the MELAS subgroup of mitochondrial encephalomyopathies. Nature 348, 651-653.

37. Sasarman, F., Antonicka, H., and Shoubridge, E.A. (2008). The A3243G tRNALeu(UUR) MELAS mutation causes amino acid misincorporation and a combined respiratory chain assembly defect partially suppressed by overexpression of EFTu and EFG2. Human molecular genetics 17, 3697-3707.

38. Desquiret-Dumas, V., Gueguen, N., Barth, M., Chevrollier, A., Hancock, S., Wallace, D.C., Amati-Bonneau, P., Henrion, D., Bonneau, D., Reynier, P., et al. (2012). Metabolically induced heteroplasmy shifting and l-arginine treatment reduce the energetic defect in a neuronal-like model of MELAS. Biochimica et biophysica acta 1822, 1019-1029.

39. Picard, M., Zhang, J., Hancock, S., Derbeneva, O., Golhar, R., Golik, P., O'Hearn, S., Levy, S., Potluri, P., Lvova, M., et al. (2014). Progressive increase in mtDNA 3243A>G heteroplasmy causes abrupt transcriptional reprogramming. Proceedings of the National Academy of Sciences of the United States of America 111, E4033-4042.

40. Janssen, A.J., Schuelke, M., Smeitink, J.A., Trijbels, F.J., Sengers, R.C., Lucke, B., Wintjes, L.T., Morava, E., van Engelen, B.G., Smits, B.W., et al. (2008). Muscle 3243A-->G mutation load and capacity of the mitochondrial energy-generating system. Annals of neurology $63,473-$ 481.

41. Holt, I.J., Harding, A.E., Petty, R.K., and Morgan-Hughes, J.A. (1990). A new mitochondrial disease associated with mitochondrial DNA heteroplasmy. American journal of human genetics 46, 428-433.

42. Nijtmans, L.G., Henderson, N.S., Attardi, G., and Holt, I.J. (2001). Impaired ATP synthase assembly associated with a mutation in the human ATP synthase subunit 6 gene. The Journal of biological chemistry 276, 6755-6762.

43. Baracca, A., Sgarbi, G., Mattiazzi, M., Casalena, G., Pagnotta, E., Valentino, M.L., Moggio, 
M., Lenaz, G., Carelli, V., and Solaini, G. (2007). Biochemical phenotypes associated with the mitochondrial ATP6 gene mutations at nt8993. Biochimica et biophysica acta 1767, 913-919.

44. Sgarbi, G., Baracca, A., Lenaz, G., Valentino, L.M., Carelli, V., and Solaini, G. (2006). Inefficient coupling between proton transport and ATP synthesis may be the pathogenic mechanism for NARP and Leigh syndrome resulting from the T8993G mutation in mtDNA. The Biochemical journal 395, 493-500.

45. Manfredi, G., Gupta, N., Vazquez-Memije, M.E., Sadlock, J.E., Spinazzola, A., De Vivo, D.C., and Schon, E.A. (1999). Oligomycin induces a decrease in the cellular content of a pathogenic mutation in the human mitochondrial ATPase 6 gene. The Journal of biological chemistry 274 , 9386-9391.

46. Tarnopolsky, M., Meaney, B., Robinson, B., Sheldon, K., and Boles, R.G. (2013). Severe infantile leigh syndrome associated with a rare mitochondrial ND6 mutation, m.14487T>C. American journal of medical genetics Part A 161A, 2020-2023.

47. Ugalde, C., Triepels, R.H., Coenen, M.J., van den Heuvel, L.P., Smeets, R., Uusimaa, J., Briones, P., Campistol, J., Majamaa, K., Smeitink, J.A., et al. (2003). Impaired complex I assembly in a Leigh syndrome patient with a novel missense mutation in the ND6 gene. Annals of neurology 54, 665-669.

48. Dermaut, B., Seneca, S., Dom, L., Smets, K., Ceulemans, L., Smet, J., De Paepe, B., Tousseyn, S., Weckhuysen, S., Gewillig, M., et al. (2010). Progressive myoclonic epilepsy as an adult-onset manifestation of Leigh syndrome due to m.14487T>C. Journal of neurology, neurosurgery, and psychiatry 81, 90-93.

49. Gonzalo, R., Garcia-Arumi, E., Llige, D., Marti, R., Solano, A., Montoya, J., Arenas, J., and Andreu, A.L. (2005). Free radicals-mediated damage in transmitochondrial cells harboring the T14487C mutation in the ND6 gene of mtDNA. FEBS letters 579, 6909-6913.

50. Fan, W., Waymire, K.G., Narula, N., Li, P., Rocher, C., Coskun, P.E., Vannan, M.A., Narula, J., Macgregor, G.R., and Wallace, D.C. (2008). A mouse model of mitochondrial disease reveals germline selection against severe mtDNA mutations. Science 319, 958-962.

51. Ge, H., Tollner, T.L., Hu, Z., Dai, M., Li, X., Guan, H., Shan, D., Zhang, X., Lv, J., Huang, C., et al. (2012). The importance of mitochondrial metabolic activity and mitochondrial DNA replication during oocyte maturation in vitro on oocyte quality and subsequent embryo developmental competence. Molecular reproduction and development 79, 392-401.

52. Fosslien, E. (2001). Mitochondrial medicine--molecular pathology of defective oxidative phosphorylation. Annals of clinical and laboratory science 31, 25-67.

53. Zhang, Y.Z., Ouyang, Y.C., Hou, Y., Schatten, H., Chen, D.Y., and Sun, Q.Y. (2008). Mitochondrial behavior during oogenesis in zebrafish: a confocal microscopy analysis. Development, growth \& differentiation 50, 189-201.

54. Zhou, R.R., Wang, B., Wang, J., Schatten, H., and Zhang, Y.Z. (2010). Is the mitochondrial cloud the selection machinery for preferentially transmitting wild-type mtDNA between generations? Rewinding Muller's ratchet efficiently. Current genetics 56, 101-107.

55. Milani, L. (2015). Mitochondrial membrane potential: a trait involved in organelle inheritance? Biol Lett 11.

56. Craven, L., Tuppen, H.A., Greggains, G.D., Harbottle, S.J., Murphy, J.L., Cree, L.M., Murdoch, A.P., Chinnery, P.F., Taylor, R.W., Lightowlers, R.N., et al. (2010). Pronuclear transfer in human embryos to prevent transmission of mitochondrial DNA disease. Nature 465, 82-85.

57. Yamada, M., Emmanuele, V., Sanchez-Quintero, M.J., Sun, B., Lallos, G., Paull, D., Zimmer, M., Pagett, S., Prosser, R.W., Sauer, M.V., et al. (2016). Genetic Drift Can Compromise Mitochondrial Replacement by Nuclear Transfer in Human Oocytes. Cell Stem Cell. 


\section{Supplemental Experimental Procedures}

Table S5.1, related to Figure 5.3 | Publications contributing heteroplasmy values m.3243A>G and $\mathrm{m} .8993 \mathrm{~T}>\mathrm{G}$ for meta-analysis.

\begin{tabular}{|c|c|c|c|c|c|}
\hline \multicolumn{3}{|c|}{ m.3243A $>$ G mutation } & \multicolumn{3}{|c|}{ m.8993T>G mutation } \\
\hline $\begin{array}{l}\text { Pedi- } \\
\text { grees }\end{array}$ & $\begin{array}{c}\text { Trans- } \\
\text { missions }\end{array}$ & Reference & $\begin{array}{l}\text { Pedi- } \\
\text { grees }\end{array}$ & $\begin{array}{c}\text { Trans- } \\
\text { missions }\end{array}$ & Reference \\
\hline 3 & 8 & (Ciafaloni et al., 1992) & 1 & 3 & (Holt et al., 1990) \\
\hline 2 & 8 & (Martinuzzi et al., 1992) & 1 & 1 & (Sakuta et al., 1992) \\
\hline 1 & 5 & (Huang et al., 1994) & 1 & 2 & (Puddu et al., 1993) \\
\hline 1 & 4 & (Liou et al., 1994) & 1 & 4 & (Shoffner et al., 1992) \\
\hline 6 & 15 & (Hammans et al., 1995) & 1 & 5 & (Tatuch et al., 1992) \\
\hline 3 & 8 & (Iwanishi et al., 1995) & 1 & 2 & (Ciafaloni et al., 1993) \\
\hline 1 & 2 & (Rusanen et al., 1995) & 4 & 6 & (Santorelli et al., 1993) \\
\hline 1 & 1 & (Fabrizi et al., 1996) & 1 & 5 & (Fryer et al., 1994) \\
\hline 1 & 3 & (Huang et al., 1996) & 1 & 1 & (Lodi et al., 1994) \\
\hline 2 & 8 & (Jansen et al., 1997) & 1 & 1 & (Pastores et al., 1994) \\
\hline 1 & 2 & (Vilarinho et al., 1997) & 1 & 6 & (Degoul et al., 1995) \\
\hline 1 & 1 & (Wilichowski et al., 1998) & 1 & 3 & (Houstek et al., 1995) \\
\hline 3 & 12 & (Olsson et al., 1998) & 1 & 8 & (Makela-Bengs et al., 1995) \\
\hline 2 & 2 & (Onishi et al., 1998) & 1 & 2 & (Tulinius et al., 1995) \\
\hline 1 & 2 & (Chinnery et al., 1999) & 1 & 3 & (Bartley et al., 1996) \\
\hline 1 & 8 & (Dubeau et al., 2000) & 1 & 3 & (Mak et al., 1996) \\
\hline 1 & 1 & (Hotta et al., 2001) & 1 & 4 & (Ferlin et al., 1997) \\
\hline 1 & 2 & (Ko et al., 2001) & 4 & 9 & (Uziel et al., 1997) \\
\hline 1 & 5 & (Lien et al., 2001) & 4 & 16 & (White et al., 1999) \\
\hline 1 & 2 & (Morovvati et al., 2002) & 1 & 2 & (Porto et al., 2001) \\
\hline 1 & 3 & (Cervin et al., 2004) & 1 & 2 & (Wong et al., 2002) \\
\hline 1 & 1 & (Chou et al., 2004) & 1 & 3 & (Enns et al., 2006) \\
\hline 1 & 1 & (Lu et al., 2002) & 1 & 3 & (Steffann et al., 2007) \\
\hline & & & 2 & 5 & (Mkaouar-Rebai et al., 2009) \\
\hline
\end{tabular}

\section{Supplemental References}

Bartley, J., Senadheera, D., Park, P., Brar, H., and Wong, L.J. (1996). Prenatal diagnosis of T8993G mitochondrial DNA point mutation in amniocytes by heteroplasmy detection. American journal of human genetics 59, A316.

Cervin, C., Liljestrom, B., Tuomi, T., Heikkinen, S., Tapanainen, J.S., Groop, L., and Cilio, C.M. (2004). Cosegregation of MIDD and MODY in a pedigree: functional and clinical consequences. Diabetes 53, 1894-1899. 
Chinnery, P.F., Zwijnenburg, P.J., Walker, M., Howell, N., Taylor, R.W., Lightowlers, R.N., Bindoff, L., and Turnbull, D.M. (1999). Nonrandom tissue distribution of mutant mtDNA. American journal of medical genetics $85,498-501$.

Chou, Y.J., Ou, C.Y., Hsu, T.Y., Liou, C.W., Lee, C.F., Tso, D.J., and Wei, Y.H. (2004). Prenatal diagnosis of a fetus harboring an intermediate load of the A3243G mtDNA mutation in a maternal carrier diagnosed with MELAS syndrome. Prenatal diagnosis 24, 367-370.

Ciafaloni, E., Ricci, E., Shanske, S., Moraes, C.T., Silvestri, G., Hirano, M., Simonetti, S., Angelini, C., Donati, M.A., Garcia, C., et al. (1992). MELAS: clinical features, biochemistry, and molecular genetics. Annals of neurology 31, 391-398.

Ciafaloni, E., Santorelli, F.M., Shanske, S., Deonna, T., Roulet, E., Janzer, C., Pescia, G., and DiMauro, S. (1993). Maternally inherited Leigh syndrome. The Journal of pediatrics 122, 419-422.

Degoul, F., Diry, M., Rodriguez, D., Robain, O., Francois, D., Ponsot, G., Marsac, C., and Desguerre, I. (1995). Clinical, biochemical, and molecular analysis of a maternally inherited case of Leigh syndrome (MILS) associated with the mtDNA T8993G point mutation. Journal of inherited metabolic disease 18, 682-688.

Dubeau, F., De Stefano, N., Zifkin, B.G., Arnold, D.L., and Shoubridge, E.A. (2000). Oxidative phosphorylation defect in the brains of carriers of the tRNAleu(UUR) A3243G mutation in a MELAS pedigree. Annals of neurology 47, 179-185.

Enns, G.M., Bai, R.K., Beck, A.E., and Wong, L.J. (2006). Molecular-clinical correlations in a family with variable tissue mitochondrial DNA T8993G mutant load. Molecular genetics and metabolism 88, 364-371.

Fabrizi, G.M., Cardaioli, E., Grieco, G.S., Cavallaro, T., Malandrini, A., Manneschi, L., Dotti, M.T., Federico, A., and Guazzi, G. (1996). The A to $G$ transition at nt 3243 of the mitochondrial tRNALeu(UUR) may cause an MERRF syndrome. Journal of neurology, neurosurgery, and psychiatry 61, 47-51.

Ferlin, T., Landrieu, P., Rambaud, C., Fernandez, H., Dumoulin, R., Rustin, P., and Mousson, B. (1997). Segregation of the G8993 mutant mitochondrial DNA through generations and embryonic tissues in a family at risk of Leigh syndrome. The Journal of pediatrics 131, 447-449.

Fryer, A., Appleton, R., Sweeney, M.G., Rosenbloom, L., and Harding, A.E. (1994). Mitochondrial DNA 8993 (NARP) mutation presenting with a heterogeneous phenotype including 'cerebral palsy'. Archives of disease in childhood 71, 419-422.

Hammans, S.R., Sweeney, M.G., Hanna, M.G., Brockington, M., Morgan-Hughes, J.A., and Harding, A.E. (1995). The mitochondrial DNA transfer RNALeu(UUR) A-->G(3243) mutation. A clinical and genetic study. Brain : a journal of neurology 118 (Pt 3), 721-734.

Holt, I.J., Harding, A.E., Petty, R.K., and Morgan-Hughes, J.A. (1990). A new mitochondrial disease associated with mitochondrial DNA heteroplasmy. American journal of human genetics 46, 428-433. Hotta, O., Inoue, C.N., Miyabayashi, S., Furuta, T., Takeuchi, A., and Taguma, Y. (2001). Clinical and pathologic features of focal segmental glomerulosclerosis with mitochondrial tRNALeu(UUR) gene mutation. Kidney international 59, 1236-1243.

Houstek, J., Klement, P., Hermanska, J., Houstkova, H., Hansikova, H., Van den Bogert, C., and Zeman, J. (1995). Altered properties of mitochondrial ATP-synthase in patients with a T-->G mutation in the ATPase 6 (subunit a) gene at position 8993 of mtDNA. Biochimica et biophysica acta 1271, 349357.

Huang, C.C., Chen, R.S., Chen, C.M., Wang, H.S., Lee, C.C., Pang, C.Y., Hsu, H.S., Lee, H.C., and Wei, Y.H. (1994). MELAS syndrome with mitochondrial tRNA(Leu(UUR)) gene mutation in a Chinese family. Journal of neurology, neurosurgery, and psychiatry $57,586-589$.

Huang, C.C., Chen, R.S., Chu, N.S., Pang, C.Y., and Wei, Y.H. (1996). Random mitotic segregation of mitochondrial DNA in MELAS syndrome. Acta neurologica Scandinavica 93, 198-202.

Iwanishi, M., Obata, T., Yamada, S., Maegawa, H., Tachikawa-Ide, R., Ugi, S., Hasegawa, M., Kojima, H., Oguni, T., Toudo, R., et al. (1995). Clinical and laboratory characteristics in the families with diabetes and a mitochondrial tRNA(LEU(UUR)) gene mutation. Diabetes research and clinical practice $29,75-82$.

Jansen, J.J., Maassen, J.A., van der Woude, F.J., Lemmink, H.A., van den Ouweland, J.M., t' Hart, L.M., Smeets, H.J., Bruijn, J.A., and Lemkes, H.H. (1997). Mutation in mitochondrial tRNA(Leu(UUR)) gene associated with progressive kidney disease. Journal of the American Society of Nephrology : JASN 8, 1118-1124.

Ko, C.H., Lam, C.W., Tse, P.W., Kong, C.K., Chan, A.K., and Wong, L.J. (2001). De novo mutation in the mitochondrial tRNALeu(UUR) gene (A3243G) with rapid segregation resulting in MELAS in the offspring. Journal of paediatrics and child health 37, 87-90.

Lien, L.M., Lee, H.C., Wang, K.L., Chiu, J.C., Chiu, H.C., and Wei, Y.H. (2001). Involvement of nervous system in maternally inherited diabetes and deafness (MIDD) with the A3243G mutation of 
mitochondrial DNA. Acta neurologica Scandinavica 103, 159-165.

Liou, C.W., Huang, C.C., Chee, E.C., Jong, Y.J., Tsai, J.L., Pang, C.Y., Lee, H.C., and Wei, Y.H. (1994). MELAS syndrome: correlation between clinical features and molecular genetic analysis. Acta neurologica Scandinavica 90, 354-359.

Lodi, R., Montagna, P., lotti, S., Zaniol, P., Barboni, P., Puddu, P., and Barbiroli, B. (1994). Brain and muscle energy metabolism studied in vivo by 31P-magnetic resonance spectroscopy in NARP syndrome. Journal of neurology, neurosurgery, and psychiatry 57, 1492-1496.

Lu, C.Y., Tso, D.J., Yang, T., Jong, Y.J., and Wei, Y.H. (2002). Detection of DNA mutations associated with mitochondrial diseases by Agilent 2100 bioanalyzer. Clinica chimica acta; international journal of clinical chemistry 318, 97-105.

Mak, S.C., Chi, C.S., Liu, C.Y., Pang, C.Y., and Wei, Y.H. (1996). Leigh syndrome associated with mitochondrial DNA 8993 T-->G mutation and ragged-red fibers. Pediatric neurology 15, 72-75.

Makela-Bengs, P., Suomalainen, A., Majander, A., Rapola, J., Kalimo, H., Nuutila, A., and Pihko, H. (1995). Correlation between the clinical symptoms and the proportion of mitochondrial DNA carrying the 8993 point mutation in the NARP syndrome. Pediatric research 37, 634-639.

Martinuzzi, A., Bartolomei, L., Carrozzo, R., Mostacciuolo, M., Carbonin, C., Toso, V., Ciafaloni, E., Shanske, S., DiMauro, S., and Angelini, C. (1992). Correlation between clinical and molecular features in two MELAS families. Journal of the neurological sciences 113, 222-229.

Mkaouar-Rebai, E., Chaari, W., Younes, S., Bousoffara, R., Sfar, M.T., and Fakhfakh, F. (2009).

Maternally inherited Leigh syndrome: T8993G mutation in a Tunisian family. Pediatric neurology 40, 437-442.

Morovvati, S., Nakagawa, M., Sato, Y., Hamada, K., Higuchi, I., and Osame, M. (2002). Phenotypes and mitochondrial DNA substitutions in families with $\mathrm{A} 3243 \mathrm{G}$ mutation. Acta neurologica Scandinavica 106, 104-108.

Olsson, C., Zethelius, B., Lagerstrom-Fermer, M., Asplund, J., Berne, C., and Landegren, U. (1998). Level of heteroplasmy for the mitochondrial mutation A3243G correlates with age at onset of diabetes and deafness. Human mutation 12, 52-58.

Onishi, H., Hanihara, T., Sugiyama, N., Kawanishi, C., Iseki, E., Maruyama, Y., Yamada, Y., Kosaka, K., Yagishita, S., Sekihara, H., et al. (1998). Pancreatic exocrine dysfunction associated with mitochondrial tRNA(Leu)(UUR) mutation. Journal of medical genetics 35, 255-257.

Pastores, G.M., Santorelli, F.M., Shanske, S., Gelb, B.D., Fyfe, B., Wolfe, D., and Willner, J.P. (1994). Leigh syndrome and hypertrophic cardiomyopathy in an infant with a mitochondrial DNA point mutation (T8993G). American journal of medical genetics 50, 265-271.

Porto, F.B., Mack, G., Sterboul, M.J., Lewin, P., Flament, J., Sahel, J., and Dollfus, H. (2001). Isolated late-onset cone-rod dystrophy revealing a familial neurogenic muscle weakness, ataxia, and retinitis pigmentosa syndrome with the T8993G mitochondrial mutation. American journal of ophthalmology 132, 935-937.

Puddu, P., Barboni, P., Mantovani, V., Montagna, P., Cerullo, A., Bragliani, M., Molinotti, C., and Caramazza, R. (1993). Retinitis pigmentosa, ataxia, and mental retardation associated with mitochondrial DNA mutation in an Italian family. The British journal of ophthalmology 77, 84-88. Rusanen, H., Majamaa, K., Tolonen, U., Remes, A.M., Myllyla, R., and Hassinen, I.E. (1995). Demyelinating polyneuropathy in a patient with the tRNA(Leu)(UUR) mutation at base pair 3243 of the mitochondrial DNA. Neurology 45, 1188-1192.

Sakuta, R., Goto, Y., Horai, S., Ogino, T., Yoshinaga, H., Ohtahara, S., and Nonaka, I. (1992). Mitochondrial DNA mutation and Leigh's syndrome. Annals of neurology 32, 597-598.

Santorelli, F.M., Shanske, S., Macaya, A., DeVivo, D.C., and DiMauro, S. (1993). The mutation at nt 8993 of mitochondrial DNA is a common cause of Leigh's syndrome. Annals of neurology 34, 827-834. Shoffner, J.M., Fernhoff, P.M., Krawiecki, N.S., Caplan, D.B., Holt, P.J., Koontz, D.A., Takei, Y., Newman, N.J., Ortiz, R.G., Polak, M., et al. (1992). Subacute necrotizing encephalopathy: oxidative phosphorylation defects and the ATPase 6 point mutation. Neurology 42, 2168-2174.

Steffann, J., Gigarel, N., Corcos, J., Bonniere, M., Encha-Razavi, F., Sinico, M., Prevot, S., Dumez, Y., Yamgnane, A., Frydman, R., et al. (2007). Stability of the m.8993T->G mtDNA mutation load during human embryofetal development has implications for the feasibility of prenatal diagnosis in NARP syndrome. Journal of medical genetics 44, 664-669.

Tatuch, Y., Christodoulou, J., Feigenbaum, A., Clarke, J.T., Wherret, J., Smith, C., Rudd, N., PetrovaBenedict, R., and Robinson, B.H. (1992). Heteroplasmic mtDNA mutation (T----G) at 8993 can cause Leigh disease when the percentage of abnormal mtDNA is high. American journal of human genetics 50, 852-858.

Tulinius, M.H., Houshmand, M., Larsson, N.G., Holme, E., Oldfors, A., Holmberg, E., and Wahlstrom, J. (1995). De novo mutation in the mitochondrial ATP synthase subunit 6 gene (T8993G) with rapid segregation resulting in Leigh syndrome in the offspring. Human genetics 96, 290-294. 
Uziel, G., Moroni, I., Lamantea, E., Fratta, G.M., Ciceri, E., Carrara, F., and Zeviani, M. (1997). Mitochondrial disease associated with the T8993G mutation of the mitochondrial ATPase 6 gene: a clinical, biochemical, and molecular study in six families. Journal of neurology, neurosurgery, and psychiatry $63,16-22$.

Vilarinho, L., Santorelli, F.M., Rosas, M.J., Tavares, C., Melo-Pires, M., and DiMauro, S. (1997). The mitochondrial A3243G mutation presenting as severe cardiomyopathy. Journal of medical genetics 34 , 607-609.

White, S.L., Shanske, S., McGill, J.J., Mountain, H., Geraghty, M.T., DiMauro, S., Dahl, H.H., and Thorburn, D.R. (1999). Mitochondrial DNA mutations at nucleotide 8993 show a lack of tissue- or agerelated variation. Journal of inherited metabolic disease 22, 899-914.

Wilichowski, E., Korenke, G.C., Ruitenbeek, W., De Meirleir, L., Hagendorff, A., Janssen, A.J., Lissens, W., and Hanefeld, F. (1998). Pyruvate dehydrogenase complex deficiency and altered respiratory chain function in a patient with Kearns-Sayre/MELAS overlap syndrome and A3243G mtDNA mutation. Journal of the neurological sciences 157, 206-213.

Wong, L.J., Wong, H., and Liu, A. (2002). Intergenerational transmission of pathogenic heteroplasmic mitochondrial DNA. Genetics in medicine : official journal of the American College of Medical Genetics 4, 78-83. 



\section{Chapter 6}

Tfam knockdown causes a developmental defect of heart and brain due to energy deficit and mitochondrial stress response

Auke BC Otten, Mike Gerards, Ellen EH Lambrichs, Sabina JV Vanherle, Florence HJ van Tienen, Patrick Lindsey, Jo M Vanoevelen, Marc Muller, Hubert JM Smeets 


\section{Summary}

A high mitochondrial DNA (mtDNA) copy number is essential for reproduction, as it correlates with both the fertility and viability of oocytes and early embryos. We investigated the effect of a reduced mtDNA copy number during early development by knocking down mitochondrial transcription factor $\mathrm{A}$ (Tfam), a key regulator of the mtDNA copy number, using morpholino oligonucleotides (MOs). Injected embryos displayed an embryonic developmental defect, especially of the heart and the brain, starting at 4 days post fertilization (dpf). In morphant zebrafish, the mtDNA copy number showed a decrease of $50-80 \%$. Using transcriptomics, we investigated the effect of Tfam knockdown on the expression of other genes and pathways at $4 \mathrm{dpf}$. Morphant zebrafish displayed downregulation of mtDNA-encoded proteins involved in electron transport chain. Regarding nuclear-encoded pathways, metabolic pathways as well as pathways important for proliferation during embryogenesis were downregulated, while we observed a significant upregulation of pathways involved in cell cycle and cell signalling pathways important for embryogenesis. Investigation of additional nuclear-encoded mitochondrial-related pathways indicated upregulation of the mitochondrial translation machinery. In line with the upregulation of the Fas pathway (stress induction of heat shock proteins), we also observed an upregulation of specific genes involved in the mitochondrial unfolded protein response, some mitochondrial matrix protease genes, as well as some key genes in apoptosis. Based on these data we conclude that Tfam knockdown causes an energy deficit during embryogenesis and an imbalance between mtDNA replication, transcription and translation, which can explain the (mitochondrial) stress response and pathology observed. The mitochondrial stress pathway described can have an important role in diseases associated with mitochondrial dysfunction

\section{Introduction}

Animal and human cells are characterized by a variable mitochondrial DNA (mtDNA) copy number. A single cell can contain several hundreds of mitochondria, each having multiple copies of mtDNA molecules, depending on the tissue type, energy requirements and, for example, exercise ${ }^{1}$. Mammalian mtDNA copy numbers are highest in oocytes, typically above 100,000 copies, but variation exists among individuals and among different species ${ }^{2}$. The high mtDNA copy number in oocytes is established by a clonal expansion of the mtDNA during oogenesis $\left({ }^{3}\right.$ and chapter $\left.3^{4}\right)$. A high mtDNA copy number appears to be important for successful fertilization and embryogenesis and, in mammals, the oocyte mtDNA copy number should be sufficient for normal vertebrate development until implantation ${ }^{3 ; 5}$. In mice, it has been demonstrated that oocytes with less than 50,000 mtDNA copies will fail to resume development after implantation ${ }^{6}$. This negative correlation between mtDNA copy number and developmental competence of embryos has also been suggested for human oocytes ${ }^{7}$.

Being a key activator of mitochondrial transcription, mitochondrial transcription factor A (Tfam), a protein of the mobility group box-family ${ }^{8}$, is crucial in the regulation of the mtDNA copy number and is essential for mtDNA replication and maintenance ${ }^{9}$. A 
direct relation between the mtDNA copy number and the total Tfam protein level ${ }^{10}$ has been demonstrated in mice embryos with a heterozygous or homozygous germline Tfam knockout ${ }^{9}$. Homozygous Tfam knockout mouse embryos had dramatic mtDNA depletion with severely reduced functioning of the electron transport chain (ETC) and died after gastrulation and implantation, while heterozygous knockout Tfam mice had moderately reduced mtDNA levels and respiratory chain deficiency, which was strongest in the developing heart ${ }^{9}$. Together, these studies indicate the importance of sufficient mtDNA copy numbers during oogenesis and embryogenesis for healthy reproduction, but the precise pathologic mechanism underlying reduced mtDNA copy numbers during embryogenesis is currently unknown.

The zebrafish Tfam protein is functionally homologous to its human counterpart and is expressed ubiquitously from the earliest stages of development ${ }^{11}$. Therefore, in the present study, we used morpholino antisense oligonucleotides (MOs) ${ }^{12}$ to establish a tfam knock-down during early embryogenesis in zebrafish. We titrated the dose of a splice-modifying $\mathrm{MO}$ affecting the translation of the tfam pre-mRNA and induced a $>50 \%$ drop in the mtDNA copy number in zebrafish embryos at 4 and 5 days post fertilization (dpf). Consistent with a critical role for tfam in embryonic development ${ }^{9}$, zebrafish embryos injected with the tfam morpholino were characterized by a developmental defect, most predominant of the heart and the brain. To further unravel the effect of reduced mtDNA replication during embryonic development, we performed global gene expression analysis.

\section{Material and Methods}

\section{Zebrafish maintenance and procedures}

Zebrafish (Danio rerio) were housed and raised in the zebrafish facility of the University of Liège as described before ${ }^{13}$. To retrieve eggs, wild-type adult male and female zebrafish were separated within the same breeding tank by a plastic divider the day before breeding. This separation was removed the next day after the light was turned on in order to allow natural mating and eggs were collected after spawning. Eggs were collected in Petri dishes containing E3 medium ${ }^{13}$ and kept at $28^{\circ} \mathrm{C}$ until microinjection. Embryos were staged as described before ${ }^{14}$.

\section{Whole-mount in situ hybridization (WISH)}

Antisense probes for WISH procedure were prepared directly from PCR amplicons using T3 RNA polymerase. PCR amplicons of the Tfam gene at both the 3' (Fwd: 5'-GAAATCAAATCGTGGGAGGA-3', Rev: 5'-ACAACATGGCGTCCTCTTT-3') and 5' (Fwd:5'-CCTTTACAGGAGGAGGAGCA-3', Rev:5'-AAAAAGCCGCACTCTCTGC-3') end of the gene were amplified. Antisense probes were generated by using a T3 promotor sequence (5'-GGATCCATTAACCCTCACTAAAGGGAGGGAA-3') attached to the reverse primer, while sense probes were generated by attaching the T3promotor sequence to the forward primer.

Zebrafish embryos and larvae of equal age were pooled and fixed in $3.7 \%$ paraformaldehyde (PFA) overnight at $4^{\circ} \mathrm{C}$. Subsequently, embryos were dehydrated 
using gradual methanol concentrations and stored in $100 \%$ methanol at $-20^{\circ} \mathrm{C}$. WISH experiments were performed as described before ${ }^{15}$. Probes (incubation concentration: $10 \mathrm{ng} / \mathrm{\mu l}$ ) were labelled with digoxigenin (DIG). Anti-DIG Fab (Roche) was used with NBT/BCIP for staining of the hybridized probes. The staining procedure was checked every 15 minutes.

\section{Knockdown of Tfam and rescue experiments}

Antisense oligonucleotide morpholino oligonucleotides (MO) were purchased from Gene Tools and micro-injected into one or two-cell stage embryos. A splice modifying $\mathrm{MO}$, targeting the boundary of exon 2 and intron 2-3 (Ensemble: ENSDART00000092009) of the zebrafish tfam gene (tfam splice-MO: 5'CGGCAGATGGAAATTTACCAGGATT-3'), was injected at concentrations of 2, 4 and $6 \mathrm{ng}$. For controlling the (toxic) effect of the MO injection, a standard control morpholino (Ctrl-MO) was injected in separate embryos (of the same batch) during each experiment at equal concentrations. All MOs were dissolved in Danieau buffer and diluted to appropriate concentrations. Before injection, $0.5 \%$ rhodamine dextran (Invitrogen) was added to the MO solution to monitor proper injection of the embryos after injection by checking the distribution of the dye using fluorescence stereomicroscopy.

\section{RNA extraction and efficacy test of splicing MO using RT-PCR}

Larvae at 2dpf, injected with either Tfam splice-MO or Ctrl-MO or non-injected controls (NIC), were grouped ( $\sim 50-60$ embryos per group) and total RNA was extracted using Trizol reagent and purified using the RNeasy Mini Kit (Qiagen), according to manufacturer's instructions. After purification, RNA concentrations were assessed by the ratio of absorbance at 260/280ng, measured on a Nanodrop spectrophotometer (Thermo Scientific). $1 \mu \mathrm{g}$ of isolated RNA was dissolved in RNAse free water; the remaining RNA was stored at $-80^{\circ} \mathrm{C}$. The dissolved RNA was denatured at $65^{\circ} \mathrm{C}$. cDNA synthesis was performed in the presence of first strand buffer (final concentration: $1 \mathrm{x}), 0.75 \mu \mathrm{g}$ oligo-dT (Invitrogen), $0.75 \mu \mathrm{g}$ random hexamer-primer (Invitrogen), $50 \mathrm{nmol}$ dNTPs (GE Healthcare Life Sciences), $1 \cup$ RNAse inhibitor (RNAsin, Promega) and $5 \mathrm{U}$ reverse transcriptase for $60 \mathrm{~min}$ at $42^{\circ} \mathrm{C}$, followed by $5 \mathrm{~min}$ at $95^{\circ} \mathrm{C}$. cDNA generation was confirmed using gel electrophoresis and subsequently stored at $-20^{\circ} \mathrm{C}$. The efficacy of the MO injection was tested by PCR-amplification of three different amplicons of increasing size using a single forward primer (5'-TGCTGGTCAAGTCATTCAGC-3') in exon 1 and reverse primers in either exon 3 (5'- CTTTTGTTCGGTGGTCAACA-3'), exon 4 (5'- TCCGAATGGCTTTTCTTTTG-3') or exon 5 (5'- CGGACTTTATCGTCCTCTGC-3').

\section{Isolation of mtDNA}

Non-injected (NIC) embryos and embryos injected with either 2 of $4 \mathrm{ng}$ tfam splice$\mathrm{MO}$ or Ctrl-MO were collected individually in sterile tubes at 2, 4 and $5 \mathrm{dpf}$ and directly frozen (without water). For mtDNA isolation, embryos were thawed and lysed for $4 \mathrm{~h}$ at $55^{\circ} \mathrm{C}$ in the presence of lysis buffer $(75 \mathrm{mM} \mathrm{NaCl}, 50 \mathrm{mM}$ and $20 \mathrm{mM}$ HEPES, supplemented with $0.4 \%$ SDS and $200 \mu$ g proteinase K (Sigma)). DNA precipitation 
was performed overnight at $-20^{\circ} \mathrm{C}$ after adding isopropanol. After centrifugation, the DNA pellet was washed with $70 \%$ ethanol and dissolved in TE buffer and directly used for quantification of the mtDNA copy number. At least 8 individual embryos were isolated per group.

\section{Quantification of mtDNA copy number}

The relative mtDNA abundance was quantified by measuring the steadystate amount of both ND1 (mtDNA NADH dehydrogenase 1, Ensemble: ENSDART00000093596) and B2M (nuclear gene beta-2-microglobulin, present on both alleles of the nDNA, Ensemble: ENSDART00000075127) using the 7900HT Fast Real-Time PCR System (Applied Biosciences). mtND1 abundance was measured by using a 157-bp amplicon (Fwd: 5'-CCACTTAATTAACCCCCTAGCC-3', Rev: 5'-ATGTTTGTGGGGGTAGACCA-3') and B2M abundance was measured using a 96-bp amplicon (Fwd: 5'- CGCCTGAAAACTACGTTCTACAC-3', Rev: 5'ACTTTCGGAGTGGCTGAAAA-3'). Amplification was performed in the presence of Sensimix Sybr Hi-Rox (Bioline) under the following conditions: 2 min $50^{\circ} \mathrm{C}, 2 \mathrm{~min}$ $95^{\circ} \mathrm{C}$, followed by 40 cycles of $15 \mathrm{~s}$ at $95^{\circ} \mathrm{C}$ (denaturation) and $1 \mathrm{~min} 60^{\circ} \mathrm{C}$ (annealing and extension). To check for non-specific amplification, a dissociation (melting) curve was generated: $15 \mathrm{~s}$ at $95^{\circ} \mathrm{C}, 15 \mathrm{~s}$ at $60^{\circ} \mathrm{C}, 15 \mathrm{~s}$ at $60^{\circ} \mathrm{C}$, cooling down to $4^{\circ} \mathrm{C}$. Samples were measured in triplicate during at least two runs. mtDNA copy number was measured using the $2^{-\Delta \Delta \mathrm{Ct}}$ method and normalized to the NIC embryos of the same age. Statistical analysis was carried out using GraphPad Prism 5.02. Groups were compared using one-way analysis of variance (ANOVA) followed by the Bonferroni multiple comparisons test. $P$-values $<0.05$ were considered significant.

\section{Microarray expression experiments and pathway analysis}

For micro-array experiments, non-injected embryos and embryos with 2 ng tfam splice-MO or Ctrl-MO were collected at $4 \mathrm{dpf}$. In total, 12 embryos from each group were individually collected in sterile tubes. RNA was isolated using the MagMax-96 for microarray kit (Ambion) according to manufacturer's instructions. Concentrations were determined using spectrophotometry on the Nanodrop 2000c (Thermo Scientific) and the Bioanalyzer (Agilent Technologies). Microarray expression analysis was performed by comparing tfam splice-MO injected and Ctrl-MO injected embryos. Analyses were performed in 2+2 dye-swap hybridizations.

200 ng RNA was labelled (Cyanine 3-CTP and Cyanine 5-CTP), fragmented and hybridized using the Low Input Quick Amp Labeling Kit, Two-colour (Ambion), according to manufacturer's instructions. After labelling, amplified cRNA samples were purified using the RNeasy Mini kit (Qiagen) and Cyanine 3 and Cyanine 5 dye concentration, RNA absorbance ratio $(260 / 280 \mathrm{~nm})$ and cRNA concentration were quantified using the nanodrop 2000C (ThermoScientific). Only samples with a yield $>0.825 \mu \mathrm{g}$ cRNA and a specific dye activity $>6.0 \mathrm{pmol} / \mu \mathrm{g}$ cRNA were used for fragmentation and hybridization. For fragmentation, $825 \mathrm{ng}$ labelled cRNA was used and the final volume was adjusted to $41.8 \mu \mathrm{l}$ with RNAse-free water, followed by the hybridization procedure, as described by the manufacturer (Ambion). Hybridization was performed on microarray slides (4x44K zebrafish V3, Agilent) using gasket slides and a hybridization chamber and incubated for $17 \mathrm{~h}$ at $65^{\circ} \mathrm{C}$ and $10 \mathrm{rpm}$ in 
the hybridization oven (Agilent Technologies). Slides were washed with Triton X-102, freshly added to the Wash Buffers. Microarray slides were scanned using a DNA Microarray scanner with Surescan High-Resolution Technology (Model 2565CA, Agilent).

Only transcripts containing a non-empty transcript ID were kept from the quantification files for the analysis. This resulted in the analysis of 43,394 transcripts out of the 45,220 available on the arrays. All genes were analysed using a multivariate Gaussian linear regression $(\operatorname{MVN}(\mu, \Sigma)$ where $\mu$ is the mean, $\Sigma$ is the covariance matrix.

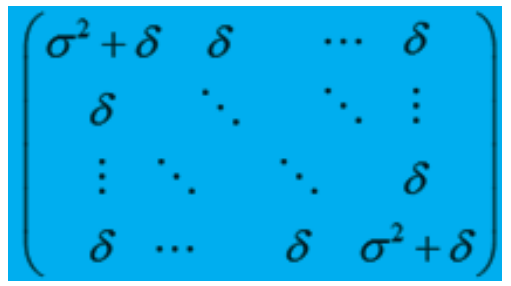

$\sigma^{2}$ is the variance, and $\delta$ is both the extra component of variance across subjects and the common covariance among responses on the same subject) including slide differences (Slide), dye swap (Dye), background level $(\mathrm{Bg})$, and a random effect. The inference criterion used for comparing the models is their ability to predict the observed data, i.e. models are compared directly through their minimized minus log-likelihood. When the numbers of parameters in models differ, they are penalized by adding the number of estimated parameters, a form of the Akaike information criterion (AIC) ${ }^{16}$.

For each transcript, a model containing the relevant covariates mentioned above $(E(y)=$ Slide $+D y e+B g)$ was fitted in order to obtain a reference AIC. Then a model containing the treatment group (Grp) was fitted $(E(y)=S l i d e+D y e+B g+G r p)$. The transcript under consideration was found to be differentially expressed if the AIC of this second model decreased compared to the model not containing the treatment. These statistical analysis were performed using the freely available program $\mathrm{R}^{17}$ and the publicly available libraries 'rmutil' and 'growth'18.

Identifiers were transformed to Ensemble gene ID codes (ENSDARG). Geneontology analysis and visualization of microarray data on biological pathways was performed using PathVisio ${ }^{19}$, with the Danio rerio (Dr_Derby_Ensembl_80) gene product/protein database. Significantly changed pathways were identified using the PathVisio software and calculated (permuted) $P$-values $<0.05$ were considered significant. Additional (mitochondrial) genes and pathways were checked by hand, after a literature search for nuclear encoded genes known to have a mitochondrial function.

\section{Results}

\section{Expression of zebrafish Tfam during zebrafish development}

We studied the expression pattern of Tfam in developing zebrafish embryos and larvae by whole-mount In Situ hybridisation (WISH) experiments in zebrafish embryos 
(Figure 6.1). Already at $24 \mathrm{hpf}$, Tfam mRNA was detected in the whole body. At $2 \mathrm{dpf}$, Tfam expression was observed with highest concentrations in the developing brain and heart region of the zebrafish embryos (Figure 6.1A-B), while the antisense probe did not show any staining (data not shown). This pattern remained at $4 \mathrm{dpf}$ (Figure 6.1C-D), with still high expression in the brain and heart region of the zebrafish embryo and, in addition, prevalent expression in the chevron-shaped somites (Figure 6.1D).

A

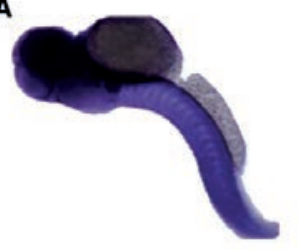

B



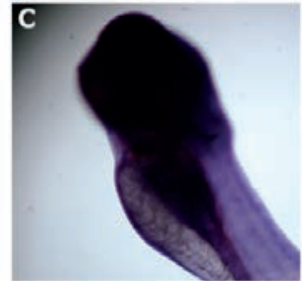

D

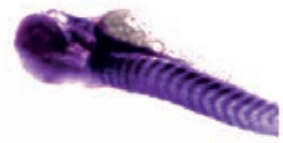

Figure 6.1 | Expression of zebrafish Tfam using whole mount in situ hybridization at A-B) 2 days post fertilization (dpf), showing expression in the whole body, with highest expression in the head; C-D) $4 \mathrm{dpf}$, showing expression in the C) head and heart region and D) whole body, especially the somites (chevron shapes).

\section{Tfam knockdown leads to specific pathologies and reduced mtDNA content}

To establish decreased mtDNA contents in zebrafish embryos we microinjected an antisense splice-MO targeting tfam-mRNA. Using RT-PCR and sequencing of tfam from total RNA extracted from MO-injected $2 \mathrm{dpf}$ embryos, we showed that exon 2 was absent from the RT-PCR product (Figure 6.2A-B). The length of exon 2 is 128 nucleotides and deletion of this exon leads to a frameshift and a premature stop codon at codon 65 (p.H29fs*35), probably leading to an absent or non-functional TFAM protein.

At $4 \mathrm{dpf}$, embryos injected with 2 or $4 \mathrm{ng}$ Tfam splice-MO displayed developmental delay compared to embryos injected with control-MO. The phenotype included overall oedema, curved tails, necrotized yolk sacs and small eyes (Figure 6.2C). Injection of $6 \mathrm{ng}$ tfam splice-MO was embryonically lethal due to severe developmental complications (Figure 6.2C). These developmental phenotypes were absent in embryos injected with control-MO. Microscopic evaluation revealed that embryos injected with $4 \mathrm{ng}$ tfam splice-MO were smaller, had a reduced brain size, as well as marked pericardial oedema (Figure 6.2 E-F), phenotypes which were absent from embryos injected with control-MO (Figure 6.2D). Furthermore, embryos injected with the splice-MO had a lower heart rate and displayed convulsive movements, although these parameters were not quantified. Strikingly, all mentioned pathologies manifested at 3 or $4 \mathrm{dpf}$, whereas development was apparently normal for tfam spliceMO injected embryos $<3 \mathrm{dpf}$.

We aimed to perform rescue experiments by injection of full-length zebrafish Tfam mRNA (100-300 pg). However, injection of zebrafish tfam mRNA appeared to result in similar (dose-dependent) phenotypes as described for the tfam splice-MO injection, suggesting that Tfam overexpression also causes pathological phenotypes. Titration of the rescue mRNA concentration was unsuccessful. 
A

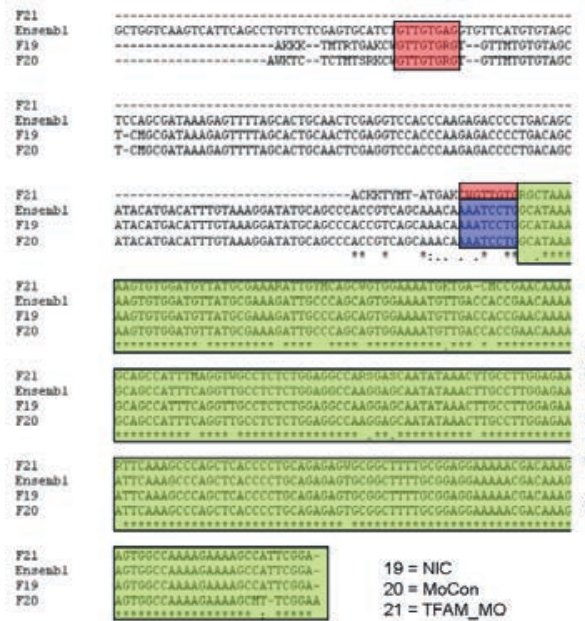

B

${ }^{\circ}$



× Normal $\square$ Dev. ret.

a Edema Brain dev. ret.

a Curved tail Small eyes

음 Necrosed yolk sac Death
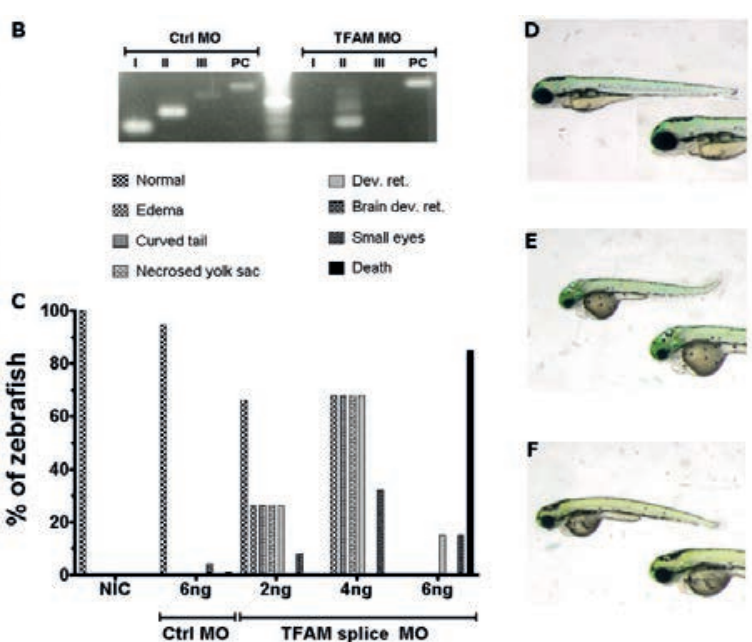

E
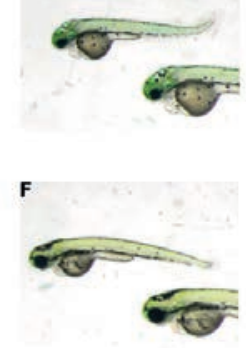

Figure 6.2 Knockdown of Tfam in zebrafish embryos. A) Ensemb/ sequence alignment of the amplified RT-PCR product II of zebrafish at 2dpf being either non-injected controls (NIC, line F19), $4 \mathrm{ng}$ Control MO injected (MoCon, line F20) and 4 ng tfam splice (e2i2, line F21) block MO injected (TFAM_ $\mathrm{MO}$ ). In red, the last nucleotides of exon 1 , in blue the last nucleotides of exon 2, which are covered by the Tfam splice-MO sequence and in green the nucleotides of exon 3. For the RT-PCR sequence of the tfam splice-block MO injected zebrafish exon 2 is completely skipped as a result of the tfam splice-MO injection, as the nucleotides of exon 1 (in red) are directly followed by the nucleotides of exon 3 (green). B) Electrophoresis gel comparing the size of three amplified Tfam transcripts of increasing lengths (I-III) and a positive control in the POLG gene (PC) in control MO-injected (Ctrl MO, 4ng) and tfam splice-MO injected (TFAM MO, 4ng) 24 hpf embryos. C) Percentage of $4 \mathrm{dpf}$ embryos with various phenotypes (see legend) when injected with 6 ng control MO (Ctrl MO) or 2, 4 or 6 ng of tfam splice block MO (TFAM splice MO). Phenotype of $4 \mathrm{dpf}$ embryos injected with D) 6 ng control MO or E-F) 4 ng tfam splice block MO.

Next, we assessed the mtDNA copy number in non-injected, control-MO injected and tfam splice-MO injected embryos. We found no difference in the mtDNA copy number in $2 \mathrm{dpf}$ embryos (Figure 6.1A). However, at $4 \mathrm{dpf}$ a significant, $\sim 50 \%$ decrease in the mtDNA copy number was observed in embryos injected with either 2 or $4 \mathrm{ng}$ Tfam splice-MO (Figure 6.3B). At $5 \mathrm{dpf}$, the effect on the mtDNA copy number was dependent on the dose of the Tfam splice-MO, causing a 50\% drop in embryos injected with 2ng Tfam splice-MO and $80 \%$ in embryos injected with 4ng Tfam splice$\mathrm{MO}$.
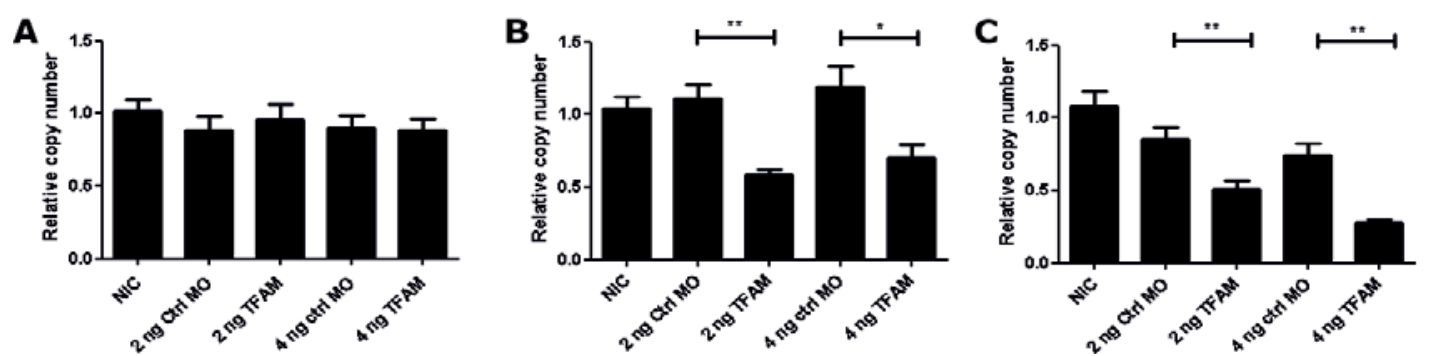

Figure 6.3 | Effect of Tfam knockdown on the mitochondrial DNA copy number. The mitochondrial DNA copy number has been assessed at A) $2 \mathrm{dpf}$, B) $4 \mathrm{dpf}$ and C) $5 \mathrm{dpf}$ in non-injected control (NIC) embryos, 2 or 4 ng control morpholino-injected (ctrl MO) embryos and 2 or 4 ng Tfam splice-block morpholino-injected (TFAM) embryos. Data are normalized to the NIC embryos. Bars indicate mean values with SEM. $P$-values are calculated using Bonferroni's Multiple Comparison Test. ${ }^{*} P$-value $<0.05$, ${ }^{* *} P$-value $<0.01$. 


\section{Differentially expressed genes in morphant zebrafish}

To evaluate the effect of Tfam knockdown on the expression of other genes, we performed global gene expression studies to compare the expression profile of $2 \mathrm{ng}$ tfam splice-MO injected, $4 \mathrm{dpf}$ embryos with the expression profile of $2 \mathrm{ng}$ control-MO injected embryos of the same age. Using two-colour microarrays, 43,394 transcripts were analysed. In total, 37,087 of these transcripts had signal intensity higher than twice the background signal. 6,683 transcripts (18\%) were differentially expressed with a fold change $>50 \%, 2,977$ transcripts were increased, while 3,706 transcripts were decreased in the tfam splice-MO injected embryos. Expression of tfam was significantly decreased (fold change (fc): 0.69 (Cl: $0.60-0.80)$ exclusively in embryos injected with the tfam splice-MO.

\section{Process-based analysis of differentially expressed genes}

Using PathVisio we identified differentially expressed pathways based on the annotated differentially expressed genes. Nine pathways were found to be significantly downregulated (genes with $\mathrm{fc}<0.67$ ) and eight pathways were significantly upregulated (genes with $\mathrm{fc}>1.5$ ) with PathVisio analysis with a z-score $>1.96$ and permute $P$-value $<0.05$ (Table 6.1). Downregulated pathways included pathways involved in (mitochondrial) metabolism, embryogenesis, physiology and cell cycle. Downregulation of the electron transport chain (ETC) pathways was mainly caused by a decrease (ND1-6, ND4L, Cytb, COXI-III, ATP6,8), while most nuclear-encoded ETC-subunits remained unchanged (Figure 6.4). Upregulated pathways included pathways involved in cell cycle, cellular signalling, important for normal embryonic development, as well as the FAS pathway and stress induction of HSP regulation.

\section{Expression of genes with mitochondrial function}

Our process-based analysis of differentially expressed pathway is restricted to those pathways annotated in the Dr_Derby_Ensembl_80 gene product/protein database, which, for zebrafish, is quite limited. Therefore, we investigated the literature ${ }^{20-30}$ for nuclear genes known to have a role in some key mitochondrial functions and looked up the expression profiles of these genes in our dataset (Table S6.1 and Table S6.2). We found a significant upregulation of many nuclear genes involved in mitochondrial translation, including genes encoding for mitochondrial ribosomal proteases $^{27}$, mitochondrial aminoacyl-tRNA synthetases ${ }^{22}$, tRNA processing and modification enzymes ${ }^{25}$, translation activators and mRNA stability factors ${ }^{20 ;} 23$ and translational initiation, elongation and termination factors ${ }^{20}$ (Table S6.1). Strikingly, none of these genes were downregulated, although some have an unchanged expression pattern. In addition, we analysed various genes encoding proteins with a role in other mitochondrial processes, including mitochondrial biogenesis and mtDNA replication proteins ${ }^{21 ; 26}$, mtDNA transcription ${ }^{28}$, protein import and activation ${ }^{26 ;}$ ${ }^{29}$, protein quality control $(P Q C)^{26}$, mitophagy ${ }^{26}$, apoptosis ${ }^{26}$ and the mitochondrial unfolded protein response (UPR) ${ }^{24 ; 30}$ (Table S6.2). Expression of genes with a role in mitochondrial biogenesis or mitochondrial transcription were largely unaffected, while 
downregulation of tfam, rnase $\mathrm{H} 1$ and the decreased mtDNA copy number suggest the process of mtDNA replication was downregulated. On the contrary, many genes involved in protein import and activation (tomm and timm genes), genes encoding matrix proteases involved in the mitochondrial PQC (e.g. lonp1, clpp), some genes encoding key proteins in the apoptosis response (e.g. casp3, casp8), as well as various genes important for the mitochondrial UPR ( $h s f 1$, chop and the mitochondrial heat shock 70 protein gene hspa9) were significantly upregulated in morphant zebrafish.

Table 6.1 | Identification of significantly downregulated $(<0.67)$ or upregulated $(>1.5)$ pathways by PathVisio analysis

\begin{tabular}{|c|c|c|c|c|c|}
\hline Pathways Danio rerio & $\begin{array}{l}\text { Changed } \\
\text { (n) }\end{array}$ & $\begin{array}{l}\text { Mea- } \\
\text { sured }(n)\end{array}$ & $\begin{array}{l}\text { Changed } \\
(\%)\end{array}$ & Z score & $\begin{array}{c}\text { P-value } \\
\text { (Permuted) }\end{array}$ \\
\hline \multicolumn{6}{|c|}{ Downregulated pathways (metabolism) } \\
\hline Glycolysis and gluconeogenesis & 13 & 27 & 48.15 & 7.12 & $<0.001$ \\
\hline $\begin{array}{l}\text { Nuclear receptors in lipid metabo- } \\
\text { lism and toxicity }\end{array}$ & 5 & 9 & 55.56 & 4.86 & $<0.001$ \\
\hline Cholesterol Biosynthesis & 5 & 9 & 55.56 & 4.86 & $<0.001$ \\
\hline Electron Transport Chain (ETC) & 12 & 68 & 17.65 & 2.51 & 0.01 \\
\hline \multicolumn{6}{|c|}{ Downregulated pathways (embryogenesis and physiology) } \\
\hline Calcium regulation in the cardiac cell & 26 & 83 & 31.33 & 7.23 & $<0.001$ \\
\hline Striated muscle contraction & 7 & 22 & 31.82 & 3.73 & 0.002 \\
\hline ACE inhibitor pathway & 3 & 7 & 42.86 & 3.11 & 0.004 \\
\hline Biogenic Amine Synthesis & 3 & 9 & 33.33 & 2.54 & 0.024 \\
\hline \multicolumn{6}{|l|}{ Downregulated pathways (Cell cycle) } \\
\hline G protein Signaling pathway & 11 & 50 & 22.00 & 3.23 & 0.006 \\
\hline \multicolumn{6}{|l|}{ Upregulated pathways (Cell cycle) } \\
\hline G1 to $S$ cell cycle control & 10 & 32 & 31.25 & 4.07 & $<0.001$ \\
\hline Cell cycle & 12 & 45 & 26.67 & 3.80 & 0.001 \\
\hline DNA replication & 7 & 23 & 30.43 & 3.31 & $<0.001$ \\
\hline Alpha6-Beta4 Integrin pathway & 9 & 41 & 21.95 & 2.60 & 0.014 \\
\hline \multicolumn{6}{|c|}{ Upregulated pathways (Embryogenesis) } \\
\hline Delta-Notch Signalling pathway & 11 & 52 & 21.15 & 2.75 & 0.005 \\
\hline Canonical Wnt signaling & 18 & 108 & 16.67 & 2.42 & 0.013 \\
\hline Endochondrial ossification & 7 & 33 & 21.21 & 2.19 & 0.016 \\
\hline \multicolumn{6}{|l|}{ Upregulated pathways (stress-related) } \\
\hline $\begin{array}{l}\text { Fas pathway and Stress induction of } \\
\text { HSP regulation }\end{array}$ & 6 & 28 & 21.43 & 2.05 & 0.036 \\
\hline
\end{tabular}

z-score values $>1.96, P$-values $<0.05$, and number changed $>2$ were considered to be significant. 


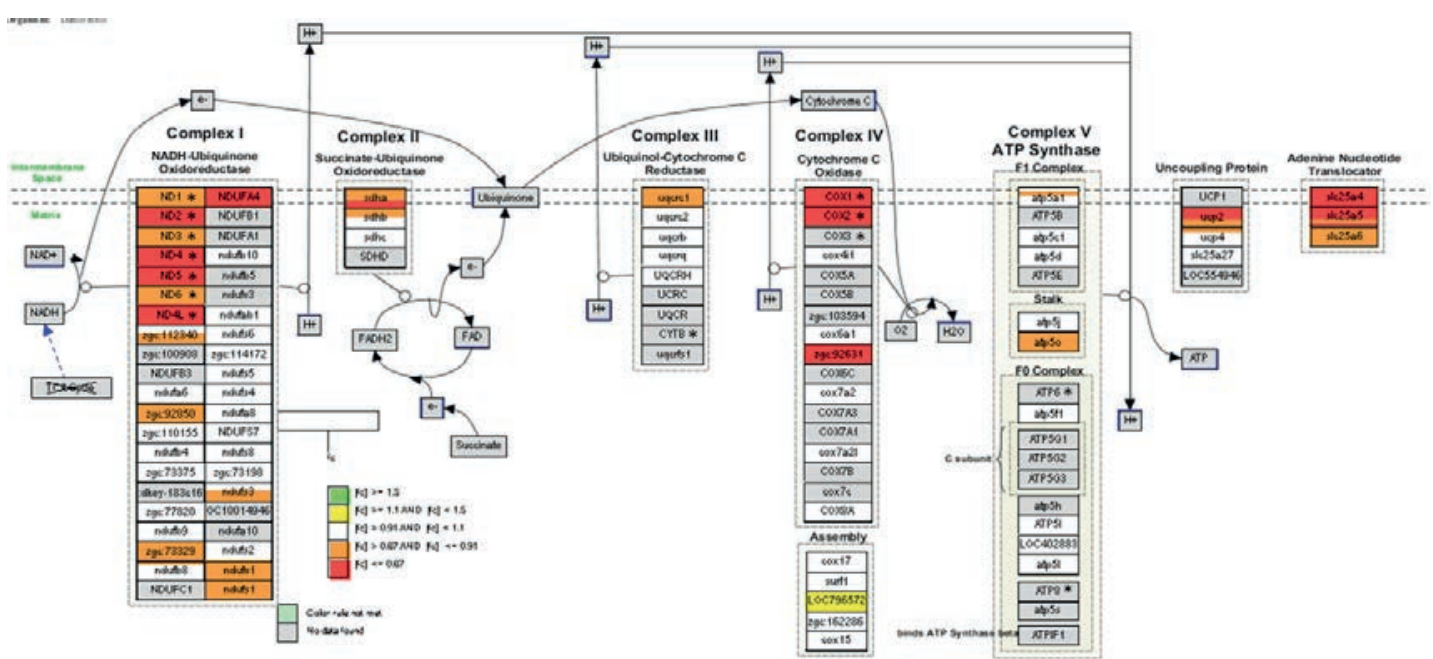

Figure 6.4 | Effect of Tfam knock-down on the expression of genes of the electron transport chain (ETC). Genes of the ETC significantly differentially expressed due to injection of tfam splice$\mathrm{MO}$. Upregulated genes are indicated in green (fold change (fc) $\geq 1.5$ ) or yellow (fc $\geq 1.1$ and $<1.5$ ). Downregulated genes are indicated in orange ( $\mathrm{fc}>0.67$ and $\leq 0.91$ ) or red (fc $\leq 0.67$ ). Genes with an unchanged expression profile are indicated in white (fc $<0.91$ and $\mathrm{fc}<1.1$ ). Genes with unknown expression profile are indicated in grey. Genes marked with an asterisk $\left({ }^{*}\right)$ lay encoded in the mtDNA.

\section{Discussion}

The main aim of this study was to investigate the role of the mtDNA copy number during zebrafish development by interfering with mtDNA replication.

\section{Specificity of the Tfam knockdown: timing of the associated phenotype}

As Tfam initiates mtDNA replication and determines the mtDNA copy number through its capability to wrap, bend and unwind the $\operatorname{mLNA}^{10}$, a function most likely conserved in zebrafish ${ }^{11}$, we established decreased mtDNA copy numbers by knocking down Tfam using morpholino oligonucleotides (MOs). Using in situ hybridizations, we observed ubiquitous expression of Tfam in embryos from 24 hours post fertilization (hpf) to $4 \mathrm{dpf}$, but expression was specifically stronger in the brain and heart. This expression pattern is consistent with previous observations during zebrafish development and indicates that Tfam is expressed ubiquitously during early zebrafish development, more preferentially in the heart, myotomes and brain ${ }^{11}$, indicating that mtDNA replication is highest during organogenesis of these organs.

To induce a Tfam knockdown, we micro-injected a splice-modifying MO targeting tfam pre-mRNA at the boundary of exon 2 and intron 2-3 into 1-4-cell stage zebrafish embryos. Sequence analysis of the resulting mRNA revealed injection of the tfam splice-MO induces skipping of exon 2, which causes a frameshift, resulting in a premature stop codon after 64 amino acids. The resulting aberrant mRNA is either eliminated through the process of nonsense-mediated mRNA decay ${ }^{31}$ or a small, alternative and probably non-functional TFAM protein is produced. In either way, the tfam splice-MO leads to a transient deficiency in functional Tfam. 
Micro-injection of MOs can potentially inflict off-target effects, not associated with loss-of-function of the target locus, e.g. through the activation of apoptosis through the $p 53$ pathway ${ }^{32}$. After injection, we found no signs of apoptosis activation following acridin orange staining. Furthermore, the observed phenotype was $\mathrm{MO}$ dose-dependent and absent in embryos injected with control-MO (the absence of any biological effect made them suitable as a control for the injection procedure). Also, the mtDNA copy number was specifically and dose-dependently decreased in embryos injected with the tfam splice-MO and not in the control-MO injected embryos. Rescue by co-injection of mRNA of the targeted gene free of the $\mathrm{MO}$ targeting sequence ${ }^{32}$ is an important validation method, but titration of the full-length zebrafish tfam-mRNA was unsuccessful and gave rise to similar phenotypes as the tfam knockdown following MO injection alone. As Tfam overexpression has been reported to cause increased mtDNA copy numbers, which is accompanied by defective transcription and deficiency of the electron transport chain ${ }^{33}$, titration of the mRNA concentration could cause a detrimental effect of tfam overexpression, prohibiting a successful rescue experiment. Nevertheless, the specific splice-effect on the mRNA and downregulation of the mtDNA copy number suggests a specific Tfam deficiency inflicted by the injection of the tfam splice-MO.

Morphant zebrafish were characterized by a $50-80 \%$ reduced mtDNA copy number and a severe developmental delay from $4 \mathrm{dpf}$ onwards. As Tfam is the main regulator of the mtDNA copy number ${ }^{9 ;}$, the effect of tfam knockdown is expected after the moment mtDNA replication initiates during embryogenesis, which, in zebrafish, is thought to be when the gastrulation stages pass into the segmentation stages (chapter $3^{4}$ ) at the end of the first day of development ${ }^{14}$. However, our and previous ${ }^{11}$ expression analysis have revealed that Tfam was already expressed at the earliest stages of zebrafish development, which is most likely due to a role for Tfam in mitochondrial transcription. It has been suggested that a strong contribution of maternal (mitochondrial) transcription genes, like tfam is made to actively transcribe mitochondrial genomes, which, on the other hand, are not replicated but passively distributed among the proliferating cells ${ }^{11}$. Developmental delay was most predominant from 4 dpf onwards, while mtDNA replication starts at the first day of development. As embryos receive a tremendous amount of mtDNA copies through their oocytes, among which high variation is observed between individual oocytes ${ }^{4}$, this suggests that many embryos can survive without or with little replication, until mtDNA copy numbers fall too short to support healthy organogenesis of the heart and brain at $4 \mathrm{dpf}$ during zebrafish development. This consistency in timing further confirms the specificity of our tfam knockdown.

\section{Deficient mtDNA replication inflicts an energy deficiency during organogenesis}

Besides the drastic decrease in mtDNA copy number, pathways with a role in mitochondrial-related energy production, including the ETC and glycolysis and gluconeogenesis, as well as pathways involved in (lipid) metabolism were specifically downregulated in morphant zebrafish. This indicates that morphant zebrafish suffer from an energetic and metabolic deficiency at a critical phase of development, when organogenesis takes place. This is also known from other models, such as mice. 
Differentiation of murine stem cells into specific cell types requires increased Tfam expression, expansion of mitochondria and mtDNA replication to meet the metabolic demands of the cell during organogenesis, while (pluripotent) stem cells can maintain a relatively low mtDNA copy number, with no expression of mtDNA replication factors, to sustain their energy requirements ${ }^{34}$. The high energy requirements for proper organogenesis is corroborated by the complete failure of organogenesis and embryonic death in mice with abolished ETC function, due to severe mtDNA depletion established through a homozygous knockdown of either $P$ olg $A^{35}$ or $\mathrm{Tfam}^{9}$, presumably because of mitochondrial insufficiency. Although we observed embryonic death only after injection of high doses of Tfam-MO at $4 \mathrm{dpf}$, this is most likely a reflection in the models used, as the use of MOs establishes a transient and possibly incomplete knock-down, which is not the case for a homozygous knockout. Bioenergetic profiling of zebrafish embryos has confirmed that zebrafish rely on an elevated mitochondrial function during organogenesis and a shift from glycolysis to the oxidative phosphorylation (OXPHOS, partly carried out by the ETC) as main energy source ${ }^{36}$. The downregulation of both the ETC and the glycolysis pathway strongly suggests a decrease in mitochondrial-related energy production in zebrafish embryos injected with tfam splice-MO. The upregulation of various pathways important for embryogenesis, including Delta-Notch ${ }^{37}$ and $\mathrm{Wnt}^{38}$, can either be considered as a compensatory response or as a reflection of the overall developmental delay in morphant zebrafish.

The observed developmental delay was most prominent of the heart and the brain. During zebrafish development, the heart and the brain are the first organs to develop ${ }^{39}$ and have a high energy demand, which makes those organs most sensitive to early embryonic deficits in energy production, as is illustrated by pericardial oedema and reduced brain size in the morphant zebrafish. The sensitivity of the developing heart to a reduced mtDNA copy number can also be explained by the requirement that $>30 \%$ of the volume of the cardiac muscle has to be occupied by mitochondria for proper functioning ${ }^{40}$. The sensitivity of the heart to the Tfam knockout has also been observed in mice heterozygous for $\mathrm{Tfam}^{9}$, which caused a respiratory chain deficiency in the heart, and in mice with disrupted Tfam in the heart and muscle ${ }^{41}$, which caused a dilated cardiomyopathy and conduction block.

\section{Imbalance between mtDNA replication and translation in morphant zebrafish}

As follow-up of the strong downregulation of pathways with a role in mitochondrial energy-production or lipid metabolism, we investigated the expression profile of other nuclear genes with a mitochondrial function (Table S6.1 and S6.2). A summary of the mitochondrial processes altered as a result of the Tfam knockdown is given in Figure 6.5. Strikingly, most nuclear-encoded genes with a role in mitochondrial translation were upregulated, including genes encoding ribosomal proteins, aminoacyl-tRNA synthethases, tRNA processing and modifying enzymes, translation activators and mRNA stability factors. Consistent with the reduced mtDNA copy number, expression of some genes with a role in mtDNA replication, tfam and rnaseh $1^{42}$, were downregulated; while genes with a role in the mtDNA nucleotide pool (sucla2, suclg1, dguok) had an unchanged expression profile. Furthermore, genes encoding 1) 
proteins of the mitochondrial protein import machinery, 2) various matrix proteases, 3) heat shock proteins and 4) some key apoptotic proteins were significantly upregulated in morphant zebrafish.

The specific upregulation of mtDNA translation in response to the Tfam knockdown creates an imbalance with the reduced mtDNA replication and transcription. As the nuclear-encoded proteins involved in mitochondrial translation are produced in the cytosol and subsequently imported into the mitochondria ${ }^{27}$, high abundance of these proteins could explain the observed upregulation of the mitochondrial import machinery. The surplus of mitochondrial proteins in the cytosol could lead to a toxic accumulation at the surface of the mitochondria ${ }^{43}$. Furthermore, the reduced presence of mitochondrial encoded ETC subunits could lead to the accumulation of orphaned nuclear encoded subunits ${ }^{24}$. Toxic accumulation of ribosomal proteins has also been documented in compounds known to induce ribotoxic stress ${ }^{44 ; 45}$.

The accumulation of unfolded or misfolded proteins can trigger a mitochondrial unfolded protein response (UPR), a mitochondrial-to-nucleus pathway in order to upregulate the expression of genes encoding chaperones and quality control proteases in an attempt to restore the mitochondrial protein homeostasis ${ }^{24 ; 46}$. In our analysis, upregulation of the Fas pathway and induction of heat shock protein synthesis suggest that a stress response is indeed activated in the morphant zebrafish (Figure 6.5). Consistent with activation of the UPR, several proteins of the 70kDa family (Hsp70) of heat shock proteins showed an increased expression profile, including HSPA9, which is the mitochondrial member of the Hsp70 family (mtHsp70 $)^{47}$. In addition, the primary transcription factor upstream of the heat shock response, heat-shock-transcriptionfactor $1(H S F 1)^{48}$, which directly regulates the gene expression of the Fas Ligand ${ }^{49}$, was significantly upregulated. Consistent with its role as important mitochondrial stress response element, the gene for transcription factor Chop ${ }^{46}$ was significantly upregulated in our analysis. CHOP regulates the expression of mitochondrial stress genes, such as Hsp60, $m t H s p 70$ and $C / p P^{46}$. ClpP, a matrix protease that primarily degrades misfolded soluble proteins ${ }^{50}$, was significantly upregulated in morphant zebrafish. Lopn1, a matrix protease with a similar function as $C l p P^{50}$ was also significantly upregulated. Altogether, these expression data suggest specific upregulation of the mitochondrial UPR in response to stress (Figure 6.5).

The factors that can activate the mitochondrial UPR can also result in the activation of the mitophagy pathway: an autophagy degradation pathway to clear severely damaged mitochondria ${ }^{24}$. In the present study, we found no clear upregulation of the mitophagy pathway. However, we did observe a significant upregulation of two key genes in the execution phase of apoptosis, casp3 and casp $8^{51}$. It has been hypothesized that mitochondrial UPR is activated prior to the mitophagy pathway and that, if mitochondrial damage becomes too pervasive, apoptosis is activated ${ }^{24}$. The upregulation of the pro-apoptotic genes casp 3 and casp 8 suggest that apoptosis is activated in the morphant zebrafish, possible as a result of the prolonged stress response, and contributes to the observed pathology as a result of Tfam knockdown (Figure 6.5).

Recently, a role of nuclear-encoded mitochondrial proteins in various cellular processes has been suggested ${ }^{43}$. The MRPL18 gene for instance, encoding a mitochondrial ribosomal protein, can also function as a protein of the cytosolic $80 \mathrm{~S}$ ribosome, required for the expression of heat shock proteins during stress conditions through $\mathrm{HSF}^{30}$. Although we did not observe an upregulation of MRPL18 in our 
analysis, the upregulation of HSF1, hsp70 proteins of the mitochondrial UPR and apoptosis, suggests upregulation of genes of the nuclear encoded mitochondrial translation might play a role in the activation of this stress response. This is corroborated by the capacity of another ribosomal gene, MRPL12, which was upregulated in our analysis, to modulate mitochondrial gene expression ${ }^{52}$.

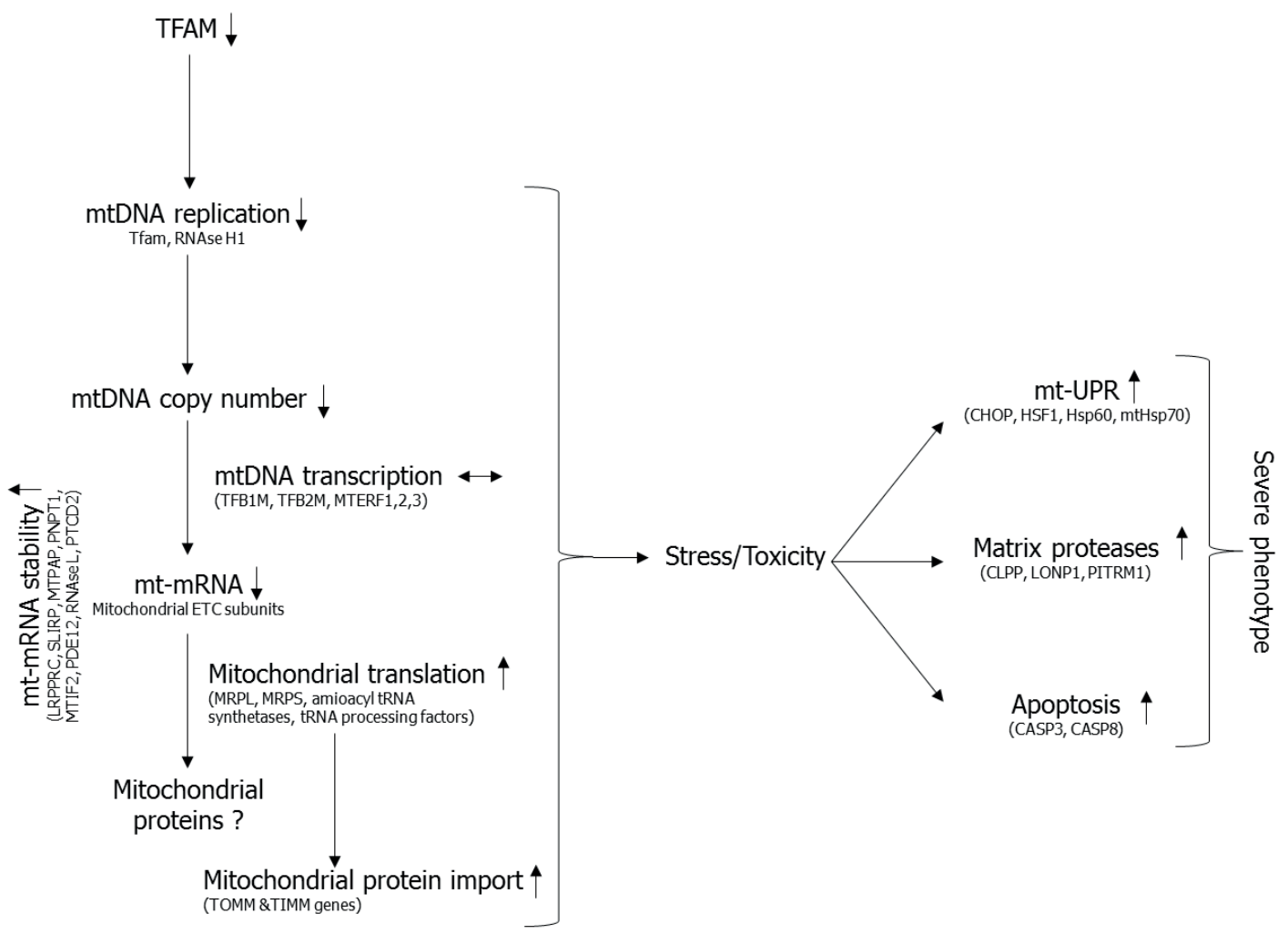

Figure 6.5 Mitochondrial processes altered as a result of Tfam based on gene expression analysis. Tfam knockdown in zebrafish causes a decrease in mtDNA replication, as indicated by the decreased mtDNA copy number in morphant zebrafish. The low mtDNA copy number, together with unchanged expression levels of mtDNA transcription factors cause a decrease in the abundance of mitochondrial mRNA transcripts, as measured by the low abundance of mitochondrial-encoded subunits of the ETC. The genes involved in the stability of these mitochondrial mRNA transcripts were upregulated, as well as many (nuclear) genes involved in mitochondrial translation and mitochondrial protein import. This imbalance between replication and translation, potential accumulation of (translation) protein at the mitochondrial membrane and the energy deficiency cause a mitochondrial stress response, as reflected by an increased mitochondrial unfolded protein response, matrix proteases and apoptosis. Together, these processes contribute to the severe phenotype of morphant zebrafish. Gene symbols represent genes involved in the implicated process. The change in gene expression induced by Tfam-knockdown is indicated with arrows; increased expression $(\uparrow)$, decreased expression $(\downarrow)$, or no change $(\rightarrow)$.

\section{Concluding remarks}

The present study shows that reduced mtDNA copy numbers during zebrafish embryogenesis leads to an energy deficiency during the critical phase of organogenesis, for which the heart and the brain are most sensitive. The energy 
deficiency causes a mitochondrial stress response, as mitochondrial- and energy-related pathways are downregulated, while the translational machinery is strongly upregulated. This confirms the concept of a tight control between the mitochondrial functional state and the cellular environment at the translational level ${ }^{43}$. Although it is not clear what stress factor initiates the mitochondrial stress response; protein accumulation, mtDNA depletion and upregulation of the translation machinery are all described to inflict such a response. Until now, the mitochondrial UPR has mainly been described in vitro. The present study shows that mitochondrial protective mechanisms are also active in vivo (Figure 6.5) and can have an important role in diseases associated with mitochondrial dysfunction.

\section{References}

1. Smeets, H.J. (2013). Preventing the transmission of mitochondrial DNA disorders: selecting the good guys or kicking out the bad guys. Reproductive biomedicine online 27, 599-610.

2. Otten, A.B., and Smeets, H.J. (2015). Evolutionary defined role of the mitochondrial DNA in fertility, disease and ageing. Human reproduction update.

3. Santos, T.A., El Shourbagy, S., and St John, J.C. (2006). Mitochondrial content reflects oocyte variability and fertilization outcome. Fertility and sterility $85,584-591$.

4. Otten, A.B., Theunissen, T.E., Lambrichs, E.H., Winandy, M., Tarbashevich, K., Raz, E., Vanoevelen, J.M., Van den Bosch, B.J., Muller, M., and Smeets, H.J. (2015). The mtDNA bottleneck size differs within and between germline and non-germline cells during zebrafish development (chapter 3). Thesis, Auke Otten.

5. Ebert, K.M., Liem, H., and Hecht, N.B. (1988). Mitochondrial DNA in the mouse preimplantation embryo. Journal of reproduction and fertility $82,145-149$.

6. Wai, T., Ao, A., Zhang, X., Cyr, D., Dufort, D., and Shoubridge, E.A. (2010). The role of mitochondrial DNA copy number in mammalian fertility. Biology of reproduction 83, 52-62.

7. Yamamoto, T., Iwata, H., Goto, H., Shiratuki, S., Tanaka, H., Monji, Y., and Kuwayama, T. (2010). Effect of maternal age on the developmental competence and progression of nuclear maturation in bovine oocytes. Molecular reproduction and development 77, 595-604.

8. Parisi, M.A., Xu, B., and Clayton, D.A. (1993). A human mitochondrial transcriptional activator can functionally replace a yeast mitochondrial HMG-box protein both in vivo and in vitro. Molecular and cellular biology 13, 1951-1961.

9. Larsson, N.G., Wang, J., Wilhelmsson, H., Oldfors, A., Rustin, P., Lewandoski, M., Barsh, G.S., and Clayton, D.A. (1998). Mitochondrial transcription factor A is necessary for mtDNA maintenance and embryogenesis in mice. Nature genetics 18, 231-236.

10. Ekstrand, M.I., Falkenberg, M., Rantanen, A., Park, C.B., Gaspari, M., Hultenby, K., Rustin, P., Gustafsson, C.M., and Larsson, N.G. (2004). Mitochondrial transcription factor A regulates mtDNA copy number in mammals. Human molecular genetics 13, 935-944.

11. Artuso, L., Romano, A., Verri, T., Domenichini, A., Argenton, F., Santorelli, F.M., and Petruzzella, V. (2012). Mitochondrial DNA metabolism in early development of zebrafish (Danio rerio). Biochimica et biophysica acta 1817, 1002-1011.

12. Pauli, A., Montague, T.G., Lennox, K.A., Behlke, M.A., and Schier, A.F. (2015). Antisense Oligonucleotide-Mediated Transcript Knockdown in Zebrafish. PloS one 10, e0139504.

13. Larbuisson, A., Dalcq, J., Martial, J.A., and Muller, M. (2013). Fgf receptors Fgfr1a and Fgfr2 control the function of pharyngeal endoderm in late cranial cartilage development. Differentiation; research in biological diversity 86, 192-206.

14. Kimmel, C.B., Ballard, W.W., Kimmel, S.R., Ullmann, B., and Schilling, T.F. (1995). Stages of embryonic development of the zebrafish. Developmental dynamics : an official publication of the American Association of Anatomists 203, 253-310.

15. Thisse, B., and Thisse, C. (2014). In situ hybridization on whole-mount zebrafish embryos and young larvae. Methods in molecular biology 1211, 53-67.

16. Akaike, H. (1973). Information theory and an extension of the maximum likelihood principle. In Second International Symposium on Inference Theory, B.N. Petrov and F. Csàki, eds. (Budapest, Akadémiai Kiadó), pp 267-281.

17. Ihaka R, G., R (1996). "R: a language for data analysis and graphics". Journal of 
Computational Graphics and Statistics 5, 299-314.

18. Lindsey, J. (1999). Models for repeated measurements, 2nd edition.(Oxford: Oxford University Press).

19. van lersel, M.P., Kelder, T., Pico, A.R., Hanspers, K., Coort, S., Conklin, B.R., and Evelo, C. (2008). Presenting and exploring biological pathways with PathVisio. BMC bioinformatics 9 , 399.

20. Boczonadi, V., and Horvath, R. (2014). Mitochondria: impaired mitochondrial translation in human disease. The international journal of biochemistry \& cell biology $48,77-84$.

21. Copeland, W.C. (2012). Defects in mitochondrial DNA replication and human disease. Critical reviews in biochemistry and molecular biology $47,64-74$.

22. Konovalova, S., and Tyynismaa, H. (2013). Mitochondrial aminoacyl-tRNA synthetases in human disease. Molecular genetics and metabolism 108, 206-211.

23. Le Roy, F., Silhol, M., Salehzada, T., and Bisbal, C. (2007). Regulation of mitochondrial mRNA stability by RNase $\mathrm{L}$ is translation-dependent and controls IFNalpha-induced apoptosis. Cell death and differentiation 14, 1406-1413.

24. Pellegrino, M.W., Nargund, A.M., and Haynes, C.M. (2013). Signaling the mitochondrial unfolded protein response. Biochimica et biophysica acta 1833, 410-416.

25. Powell, C.A., Nicholls, T.J., and Minczuk, M. (2015). Nuclear-encoded factors involved in post-transcriptional processing and modification of mitochondrial tRNAs in human disease. Frontiers in genetics 6, 79 .

26. Quiros, P.M., Langer, T., and Lopez-Otin, C. (2015). New roles for mitochondrial proteases in health, ageing and disease. Nature reviews Molecular cell biology 16, 345-359.

27. Sylvester, J.E., Fischel-Ghodsian, N., Mougey, E.B., and O'Brien, T.W. (2004). Mitochondrial ribosomal proteins: candidate genes for mitochondrial disease. Genetics in medicine : official journal of the American College of Medical Genetics 6, 73-80.

28. Taanman, J.W. (1999). The mitochondrial genome: structure, transcription, translation and replication. Biochimica et biophysica acta 1410, 103-123.

29. Wiedemann, N., Frazier, A.E., and Pfanner, N. (2004). The protein import machinery of mitochondria. The Journal of biological chemistry 279, 14473-14476.

30. Zhang, X., Gao, X., Coots, R.A., Conn, C.S., Liu, B., and Qian, S.B. (2015). Translational control of the cytosolic stress response by mitochondrial ribosomal protein L18. Nature structural \& molecular biology 22, 404-410.

31. Baker, K.E., and Parker, R. (2004). Nonsense-mediated mRNA decay: terminating erroneous gene expression. Current opinion in cell biology 16, 293-299.

32. Bedell, V.M., Westcot, S.E., and Ekker, S.C. (2011). Lessons from morpholino-based screening in zebrafish. Briefings in functional genomics 10, 181-188.

33. Ylikallio, E., Tyynismaa, H., Tsutsui, H., Ide, T., and Suomalainen, A. (2010). High mitochondrial DNA copy number has detrimental effects in mice. Human molecular genetics 19, 2695-2705.

34. Facucho-Oliveira, J.M., Alderson, J., Spikings, E.C., Egginton, S., and St John, J.C. (2007). Mitochondrial DNA replication during differentiation of murine embryonic stem cells. Journal of cell science 120, 4025-4034.

35. Hance, N., Ekstrand, M.I., and Trifunovic, A. (2005). Mitochondrial DNA polymerase gamma is essential for mammalian embryogenesis. Human molecular genetics 14, 1775-1783.

36. Stackley, K.D., Beeson, C.C., Rahn, J.J., and Chan, S.S. (2011). Bioenergetic profiling of zebrafish embryonic development. PloS one 6, e25652.

37. Shawber, C.J., and Kitajewski, J. (2004). Notch function in the vasculature: insights from zebrafish, mouse and man. BioEssays : news and reviews in molecular, cellular and developmental biology 26, 225-234.

38. Munoz-Descalzo, S., Hadjantonakis, A.K., and Arias, A.M. (2015). Wnt/ss-catenin signalling and the dynamics of fate decisions in early mouse embryos and embryonic stem (ES) cells.

Seminars in cell \& developmental biology 47-48, 101-109.

39. Glickman, N.S., and Yelon, D. (2002). Cardiac development in zebrafish: coordination of form and function. Seminars in cell \& developmental biology 13, 507-513.

40. Ventura-Clapier, R., Garnier, A., Veksler, V., and Joubert, F. (2011). Bioenergetics of the failing heart. Biochimica et biophysica acta 1813, 1360-1372.

41. Wang, J., Wilhelmsson, H., Graff, C., Li, H., Oldfors, A., Rustin, P., Bruning, J.C., Kahn, C.R., Clayton, D.A., Barsh, G.S., et al. (1999). Dilated cardiomyopathy and atrioventricular conduction blocks induced by heart-specific inactivation of mitochondrial DNA gene expression. Nature genetics 21, 133-137.

42. Reyes, A., Melchionda, L., Nasca, A., Carrara, F., Lamantea, E., Zanolini, A., Lamperti, C., 
Fang, M., Zhang, J., Ronchi, D., et al. (2015). RNASEH1 Mutations Impair mtDNA Replication and Cause Adult-Onset Mitochondrial Encephalomyopathy. American journal of human genetics 97, 186-193.

43. Richter-Dennerlein, R., Dennerlein, S., and Rehling, P. (2015). Integrating mitochondrial translation into the cellular context. Nature reviews Molecular cell biology 16, 586-592.

44. Katika, M.R., Hendriksen, P.J., Shao, J., van Loveren, H., and Peijnenburg, A. (2012). Transcriptome analysis of the human T lymphocyte cell line Jurkat and human peripheral blood mononuclear cells exposed to deoxynivalenol (DON): New mechanistic insights. Toxicology and applied pharmacology 264, 51-64.

45. Schmeits, P.C., Katika, M.R., Peijnenburg, A.A., van Loveren, H., and Hendriksen, P.J. (2014). DON shares a similar mode of action as the ribotoxic stress inducer anisomycin while TBTO shares ER stress patterns with the ER stress inducer thapsigargin based on comparative gene expression profiling in Jurkat T cells. Toxicology letters 224, 395-406.

46. Zhao, Q., Wang, J., Levichkin, I.V., Stasinopoulos, S., Ryan, M.T., and Hoogenraad, N.J. (2002). A mitochondrial specific stress response in mammalian cells. The EMBO journal 21, 4411-4419.

47. Dores-Silva, P.R., Barbosa, L.R., Ramos, C.H., and Borges, J.C. (2015). Human mitochondrial Hsp70 (mortalin): shedding light on ATPase activity, interaction with adenosine nucleotides, solution structure and domain organization. PloS one 10, e0117170.

48. Trinklein, N.D., Murray, J.I., Hartman, S.J., Botstein, D., and Myers, R.M. (2004). The role of heat shock transcription factor 1 in the genome-wide regulation of the mammalian heat shock response. Molecular biology of the cell 15, 1254-1261.

49. Bouchier-Hayes, L., McBride, S., van Geelen, C.M., Nance, S., Lewis, L.K., Pinkoski, M.J., and Beere, H.M. (2010). Fas ligand gene expression is directly regulated by stress-inducible heat shock transcription factor-1. Cell death and differentiation 17, 1034-1046.

50. Tatsuta, T., and Langer, T. (2009). AAA proteases in mitochondria: diverse functions of membrane-bound proteolytic machines. Research in microbiology 160, 711-717.

51. Harrington, H.A., Ho, K.L., Ghosh, S., and Tung, K.C. (2008). Construction and analysis of a modular model of caspase activation in apoptosis. Theoretical biology \& medical modelling 5 , 26.

52. Wang, Z., Cotney, J., and Shadel, G.S. (2007). Human mitochondrial ribosomal protein MRPL12 interacts directly with mitochondrial RNA polymerase to modulate mitochondrial gene expression. The Journal of biological chemistry 282, 12610-12618. 


\section{Supplemental Information}

\section{Table S6.1 | Expression profile of genes in the process of mitochondrial translation}

\begin{tabular}{|c|c|c|c|}
\hline Gene & Fold Change & Gene & Fold Change \\
\hline \multicolumn{2}{|c|}{ Mitochondrial ribosomal proteins } & MRPS22 & $1.29(1.06-1.56)$ \\
\hline MRPL1 & NA & MRPS23 & $1.18(1.01-1.38)$ \\
\hline MRPL2 & NA & MRPS24 & 1.0 \\
\hline MRPL3 & $1.35(1.16-1.57)$ & MRPS25 & 1.0 \\
\hline MRPL4 & NA & MRPS26 & NA \\
\hline MRPL9 & $1.68(1.52-1.86)$ & MRPS27 & NA \\
\hline MRPL10 & $1.29(1.15-1.46)$ & MRPS28 & NA \\
\hline MRPL11 & $1.21(1.06-1.39)$ & MRPS31 & $3.11(1.43-6.74)$ \\
\hline MRPL12 & $1.36(1.17-1.58)$ & MRPS33 & $1.51(1.32-1.73)$ \\
\hline MRPL13 & $1.30(1.14-1.49)$ & MRPS34 & 1.0 \\
\hline MRPL14 & $1.34(1.13-1.59)$ & MRPS35 & NA \\
\hline MRPL15 & 1.0 & MRPS36 & NA \\
\hline MRPL16 & NA & MRPS37 & NA \\
\hline MRPL17 & 1.0 & \multicolumn{2}{|c|}{ Mitochondrial aminoacyl-tRNA synthetases } \\
\hline MRPL18 & NA & AARS2 & 1.0 \\
\hline MRPL19 & 1.0 & DARS2 & NA \\
\hline MRPL20 & 1.0 & EARS2 & $1.31(1.24-1.40)$ \\
\hline MRPL21 & NA & FARS2 & 1.0 \\
\hline MRPL22 & $1.47(1.29-1.67)$ & HARS2 & $1.31(1.17-1.47)$ \\
\hline MRPL23 & 1.0 & MARS2 & 1.0 \\
\hline MRPL24 & 1.0 & RARS2 & $1.35(1.16-1.58)$ \\
\hline MRPL27 & $1.54(1.33-1.79)$ & SARS2 & $1.38(1.14-1.65)$ \\
\hline MRPL28 & $1.24(1.04-1.47)$ & YARS2 & NA \\
\hline MRPL30 & NA & GARS & NA \\
\hline MRPL31 & NA & KARS & NA \\
\hline MRPL32 & NA & \multicolumn{2}{|c|}{ tRNA processing and modification enzymes } \\
\hline MRPL33 & NA & $\begin{array}{l}\text { TRMT10C, } \\
\text { MRPP1 }\end{array}$ & $1.46(1.24-1.70)$ \\
\hline MRPL34 & NA & $\begin{array}{l}\text { HSD17B10, } \\
\text { MRPP2 }\end{array}$ & 1.0 \\
\hline MRPL35 & $1.22(1.07-1.39)$ & MRPP3 & NA \\
\hline MRPL36 & $1.37(1.10-1.69)$ & ELAC2 & $1.46(1.31-1.62)$ \\
\hline MRPL37 & $1.35(1.14-1.61)$ & PNPT1 & NA \\
\hline MRPL38 & $1.27(1.04-1.55)$ & TRNT1 & $1.46(1.32-1.63)$ \\
\hline MRPL39 & $1.38(2.24-1.55)$ & TRMT5 & NA \\
\hline MRPL40 & 1.0 & TRMT61B & NA \\
\hline MRPL41 & 1.0 & PUS1 & $1.42(1.17-1.73)$ \\
\hline MRPL42 & NA & GTPBP3 & $2.15(1.83-2.52)$ \\
\hline MRPL43 & NA & MTO1 & $1.50(1.34-1.67)$ \\
\hline MRPL44 & $1.54(1.32-1.79)$ & CDK5RAP1 & 1.0 \\
\hline
\end{tabular}


Tfam knockdown causes a developmental defect due to energy deficit and mitochondrial stress response

\begin{tabular}{|l|l|l|l|}
\hline MRPL45 & $1.69(1.47-1.95)$ & MTU1, TRMU & 1.0 \\
\hline MRPL46 & $1.24(1.05-1.47)$ & TRIT1 & $1.36(1.19-1.55)$ \\
\hline MRPL47 & NA & MTFMT & $1.10(1.01-1.20)$ \\
\hline MRPL48 & $1.43(1.25-1.62)$ & \multicolumn{1}{|l|}{ Translation activators, mRNA stability factors } \\
\hline MRPL49-57 & NA & LRPPRC & $1.47(1.23-1.74)$ \\
\hline MRPS2 & NA & SLIRP & NA \\
\hline MRPS5 & $1.20(1.03-1.38)$ & TACO1 & NA \\
\hline MRPS6 & 1.0 & MTPAP & 1.0 \\
\hline MRPS7 & 1.0 & PNPT1 & NA \\
\hline MRPS9 & NA & MTIF2 & $1.47(1.03-2.11)$ \\
\hline MRPS10 & NA & RNAse L & No homologue \\
\hline MRPS11 & $1.26(1.10-1.45)$ & PDE12 & $1.24(1.13-1.35)$ \\
\hline MRPS14 & $1.24(1.04-1.47)$ & PTCD2 & 1.0 \\
\hline MRPS15 & NA & Initiation, elongation and termination factors \\
\hline MRPS16 & NA & RMND1 & 1.0 \\
\hline MRPS17 & NA & TUFM & 1.0 \\
\hline MRPS18A & $1.41(1.18-1.69)$ & TSFM & NA \\
\hline MRPS18B & $1.38(1.10-1.72)$ & GFM1 & 1.0 \\
\hline MRPS18C & 1.0 & C12orf65 & 1.0 \\
\hline MRPS21 & NA & & \\
\hline
\end{tabular}

Data are means with confidence intervals (CI). The colours refer to the expression profile of the genes, which were either upregulated (green), downregulated (red) or unchanged (white). Genes in grey were not on the microarray. 
Table S6.2 | Expression profile of genes with a function in some key mitochondrial-related processes

\begin{tabular}{|c|c|c|c|}
\hline Gene & Fold Change & Gene & Fold Change \\
\hline \multicolumn{2}{|c|}{$\begin{array}{l}\text { Biogenesis and mtDNA replication and stability } \\
\text { factors }\end{array}$} & \multicolumn{2}{|c|}{ Protein quality control } \\
\hline POLG1 & NA & $\begin{array}{l}\text { MEP, } \\
\text { NLN }\end{array}$ & 1.0 \\
\hline POLG2 & NA & PITRM1 & $1.31(1.15-1.49)$ \\
\hline PAPOLG & 1.0 & YME1L1 & 1.0 \\
\hline CPEO & NA & SPG7 & $0.86(0.79-0.93)$ \\
\hline RNAse $\mathrm{H} 1$ & $0.92(0.84-0.99)$ & AFG3L2 & $0.86(0.76-0.95)$ \\
\hline DGUOK & 1.0 & $\begin{array}{l}\text { ATP23, XRC- } \\
\text { C6BP1 }\end{array}$ & 1.0 \\
\hline$\overline{T P}$ & $N / A$ & HTRA2 & $1.52(1.30-1.77)$ \\
\hline TK2 & NA & CLPP & $1.23(1.15-1.32)$ \\
\hline RRM2B & 1.0 & LONP1 & $1.60(1.33-1.93)$ \\
\hline MPV17 & 1.0 & PARK7 & $0.72(0.58-0.89)$ \\
\hline SUCLA2 & 1.0 & \multicolumn{2}{|l|}{ Mitophagy } \\
\hline SUCLG1 & 1.0 & HTRA2 & $1.52(1.30-1.77)$ \\
\hline OPA1 & 1.0 & USP8 & 1.0 \\
\hline ENDOG & 1.0 & USP15 & 1.0 \\
\hline SSBP1 & 1.0 & USP30 & 1.0 \\
\hline PGC1A & 1.0 & OMA1 & $0.85(0.77-0.93)$ \\
\hline NRF1 & 1.0 & PARL & $1.18(1.06-1.32)$ \\
\hline YME1L1 & 1.0 & PARK7 & $0.72(0.58-0.89)$ \\
\hline SPG7 & $0.86(0.76-0.95)$ & SENP2 & NA \\
\hline OSGEPL & $1.47(1.26-1.71)$ & SENP5 & 1.0 \\
\hline LONP1 & $1.60(1.33-1.93)$ & \multicolumn{2}{|l|}{ Apoptosis } \\
\hline \multicolumn{2}{|c|}{ Mitochondrial DNA transcription } & OMA1 & $0.85(0.77-0.93)$ \\
\hline TFB1M & 1.0 & HTRA2 & $1.52(1.30-1.77)$ \\
\hline TFB2M & 1.0 & PARL & $1.18(1.06-1.32)$ \\
\hline MTERF1 & 1.0 & CAPN1 & $0.78(0.71-0.85)$ \\
\hline MTERF2 & 1.0 & CAPN2 & 1.0 \\
\hline MTERF3 & $1.26(1.01-1.56)$ & CAPN10 & NA \\
\hline \multicolumn{2}{|c|}{ Protein import and activation } & CASP2 & 1.0 \\
\hline TOMM5 & 1.0 & CASP3a & $1.38(1.25-1.52)$ \\
\hline TOMM20a & $1.38(1.15-1.66)$ & CASP3b & $2.39(1.06-1.81)$ \\
\hline TOMM20b & 1.0 & CASP7 & 1.0 \\
\hline TOMM22 & $1.32(1.10-1.60)$ & CASP8 & $2.28(1.81-2.62)$ \\
\hline TOMM34 & 1.0 & CASP9 & 1.0 \\
\hline TOMM40 & 1.0 & USP9X & 1.0 \\
\hline TOMM70a & $1.50(1.27-1.77)$ & GZMA & 1.0 \\
\hline TIMM8a & $1.33(1.11-1.60)$ & GZMB & NA \\
\hline
\end{tabular}


Tfam knockdown causes a developmental defect due to energy deficit and mitochondrial stress response

\begin{tabular}{|l|l|l|l|}
\hline TIMM8b & $1.32(1.20-1.47)$ & \multicolumn{2}{l|}{$\begin{array}{l}\text { Mitochondrial Unfolded protein response } \\
\text { UPR })\end{array}$} \\
\hline TIMM9 & $1.44(1.28-1.63)$ & HSF1 & $1.69(1.26-2.20)$ \\
\hline TIMM10 & $1.48(1.27-1.72)$ & CHOP, ddit3 & $1.57(1.31-1.88)$ \\
\hline TIMM13 & 1.0 & $\begin{array}{l}\text { HSPA1A,B, } \\
\text { hSC70 }\end{array}$ & $0.28(0.23-0.34)$ \\
\hline TIMM17a & 1.0 & HSPA1L & 1.0 \\
\hline TIMM17b & 1.0 & HSPA2 & No homologue \\
\hline TIMM23 & $0.81(0.75-0.89)$ & HSPA4 & $1.47(1.21-1.79)$ \\
\hline TIMM44 & $1.24(1.02-1.51)$ & HSPA4L & NA \\
\hline TIMM50 & $1.40(1.17-1.68)$ & HSPA5 & $1.65(1.43-1.92)$ \\
\hline MIP & 1.0 & HSPA6 & No homologue \\
\hline PMPCB & $1.20(1.07-1.34)$ & HSPA7 & No homologue \\
\hline METAP1D & $1.24(1.11-1.39)$ & HSPA8 & 1.0 \\
\hline XPNPEP3 & 1.0 & $\begin{array}{l}\text { HSPA9, } \\
\text { mHSP70 }\end{array}$ & $1.71(1.51-1.93)$ \\
\hline IMMP2L & 1.0 & HSPA12A & 1.0 \\
\hline IMMP1L & NA & HSPA14 & $1.77(1.54-2.04)$ \\
\hline PMPCA & $1.41(1.19-1.67)$ & HSDP1/hsp60 & 1.0 \\
\hline
\end{tabular}

Data are means with confidence intervals (CI). The colours refer to the expression profile of the genes, which were either upregulated (green), downregulated (red) or unchanged (white). Genes in grey were not on the microarray. 



\section{Chapter 7}

\section{General}

\section{Discussion}

Auke BC Otten, Marc Muller, Hubert JM Smeets

Partly published Hum Reprod Update 2015 Sep;21(5):671-89 


\section{Introduction}

Mitochondria are fundamental to the origin of (multicellular) eukaryotes and provide the eukaryotes, amongst others, with the capability of generating energy, at the cost of a high mitochondrial DNA (mtDNA) mutation rate. This is partly due to the production of reactive oxygen species (ROS). The high mtDNA mutation rate has resulted in endosymbiotic gene transfer (EGT): most genes of the ancestral prokaryotic genome the mtDNA descends from have been transferred to the nucleus. As a result, the modern animal mtDNA is a small multicopy genome, containing only 13 protein-coding genes. The mtDNA mutation rate determines partly the capacity of a species to adapt to changing energy requirements in new environments. As a result, the mtDNA is crucial for both individual health and survival of a species ${ }^{1}$.

The high mtDNA mutation rate induces an evolutionary balanced introduction of deleterious, neutral and advantageous mtDNA mutations. While the advantageous mutations allow adaptation of the species, the deleterious mutation can result in mtDNA diseases ${ }^{1}$. In animals, the development of a high mtDNA copy number in combination with a purifying bottleneck during transmission has further limited the impact of mtDNA mutations. This reduces the effect of the mutations and removes low copy variants during transmission and a presumably healthy mitochondrial system will be maintained ${ }^{2}$. Nevertheless, mtDNA mutations occur and can result in devastating diseases in an individual. The way mitochondrial systems have evolved to cope with the high mtDNA mutations load in animals and humans also affects their reproduction and the way mtDNA diseases are being transmitted ${ }^{3-5}$. As a consequence, parameters associated with the mtDNA have an impact on mammalian fertility ${ }^{6}$ and reproductive options for carriers of mtDNA diseases.

In the first part of this chapter, we will discuss the role of a high mtDNA copy number and the purifying mtDNA bottleneck in the development of mitochondrial diseases and fertility. In the second part, we will discuss how the zebrafish model can be utilized to better understand the impact of mtDNA mutations, their inheritance through the mtDNA bottleneck, as well as to test interventions to ameliorate or cure mtDNA diseases.

\section{The role of mtDNA evolutionary mechanisms in disease and reproduction}

\section{Human mitochondrial diseases: genetic counseling}

Incomplete EGT and nuclear gene replacements ${ }^{7}$ have led to the existence of two genomes in a eukaryotic cell: nuclear and mitochondrial DNA. Consequently, two genetically distinct types of mitochondrial diseases exist (chapter 2). Defects in nuclear mitochondrial genes [which either have been transferred to the nuclear DNA during EGT (such as Twinkle) or have been replaced by gene exchange from other sources, like viruses (such as Polg)] lead to Mendelian diseases ${ }^{3}$. On the contrary, defects in the mtDNA genes (37 in mammals) lead to maternally inherited or sporadic de novo mtDNA diseases (at a frequency of 1 in 5,000 individuals ${ }^{8}$ ). Currently, over 
150 pathogenic mtDNA mutations have been identified ${ }^{9}$. The bigenomic origin of mitochondrial proteins complicates the genetic diagnosis of mitochondrial diseases, which are clinically highly variable, and for which, in most cases, no clear genotypephenotype correlations exist (Figure 1.1 in chapter 1). To identify mutations, genomewide diagnostic procedures, like mtDNA sequencing in combination with whole-exome sequencing are currently taking the stage. However, without a diagnosis based on a confirmed genetic defect, genetic counseling remains challenging ${ }^{10}$.

The nature of the genetic defect in either the mtDNA or nDNA has implications for the prognosis and transmission of the disorder and for the reproductive choices that patients or carriers have. Prenatal diagnosis (PND) is the preferred option for prevention of the transmission of nuclear mutations, but for mitochondrial disorders caused by heteroplasmic mtDNA mutations, PND is often unreliable, as prediction of the clinical phenotype from the heteroplasmy level in chronic villus sampling (CVS) or amniocytes is uncertain. Two exceptions exist. First, mothers of patients with a de novo mutation, which is fairly common for mtDNA disorders (see below and chapter 4) have a very low recurrence risk ${ }^{11}$ and PND can be offered for reassurance. Secondly, carriers of low level mtDNA mutations, that display skewing can be offered PND, as it is likely that the vast majority of the embryos will be mutations-free ${ }^{12 ; 13}$.

Current alternatives for preventing the transmission of mtDNA disorders are oocyte donation and preimplantation genetic diagnosis (PGD). During PGD for mtDNA disorders (Figure 7.1C), oocytes are fertilized in vitro, biopsied at the blastomeric stage and only embryos without mutation or with a mutation load below the threshold of clinical expression are transferred to the uterus ${ }^{14}$. For most mtDNA mutations, insufficient data exists to define a mutation-specific threshold. Recently, this has been overcome by a meta-analysis, which showed a $95 \%$ or higher chance of being unaffected at a (muscle) mutant level of $18 \%$ or less, irrespective of the mutation; 14. This opens up PGD for all heteroplasmic mtDNA mutation carriers who produce oocytes with mutation loads below this threshold. This is obviously not the case for homoplasmic mutations and for carriers with a very high mutation load of non-skewing mutations. A possible future reproductive option in mtDNA disorders is nuclear genome transfer (NGT; Figure 7.1D), a procedure during which the nuclear genome from an mtDNA mutation-harboring zygote or oocyte is transferred to an enucleated acceptor, harboring mutation-free mtDNA ${ }^{15}$. This would be a solution for all mtDNA mutations, including the homoplasmic mutations. As a result, the offspring would be, apart from some carry-over, mtDNA mutation-free, while still harboring the nuclear DNA from the mother. A number of studies in poor quality human rest embryos ${ }^{15}$ or animal models, like mice ${ }^{16 ; 17}$ and apes ${ }^{18}$ have demonstrated that functional replacement of the mitochondrial genome is possible, either by transfer of the pronucleus ${ }^{15}$, the spindle transfer ${ }^{19}$ or the polar body ${ }^{17}$. In the U.K., an approval of regulations to start a trial of NGT to prevent the transmission of mtDNA disorders has subsequently been issued, after careful consideration of the technical, ethical and safety issues by the U.K. Human Fertilization and Embryology Authority (HFEA) ${ }^{20}$ and the Nuffield Council on Bioethics ${ }^{21}$. Prior to a clinical trial, a further preclinical study had to be performed to solve remaining issues on safety, efficacy and feasibility, involving 500 healthy eggs from 64 donor women ${ }^{22}$. This resulted in a protocol optimized for early pronuclear transfer (ePNT; shortly after completion of meiosis), vitrification, manipulation and medium, which generates apparently normal blastocysts with a low carry-over of less than $2 \%$ in the vast majority. No effect on aneuploidy or gene expression is induced 
by the method ${ }^{22}$. This procedure, if positively evaluated by the HFEA, seems ready for clinical application, which would provide the definite proof ${ }^{23}$.

Carry-over of mutated mtDNA during the procedure is a critical issue, as this could generate a risk for developing mtDNA disease in the offspring. In most cases, the carry-over is extremely low. Nevertheless, a study in human cells showed that even small amounts of mtDNA carried over can lead to a complete conversion from heteroplasmy levels around $1 \%$ to homoplasmy ${ }^{24}$. In ePNT carry-over was less than $2 \%$, but in one of five blastocyst derived ES cell lines a continuous increase in carry-over mtDNA was observed ${ }^{22}$. This indicates that in a situation of mixed mtDNA templates, which can result in altered mitochondrial function ${ }^{25}$, either genetic drift or a replication or selective advantage (as described in chapter 5), may occur. This implies that mitochondrial replacement does not fully rule out mtDNA disease in the offspring, but is still more a risk reduction strategy ${ }^{22}$. Reducing heteroplasmy in mitochondrial replacement or preselecting mtDNA backgrounds based on replication differences could further minimize this risk.

Another important concern for applying mitochondrial replacement is the withdrawal of the mtDNA from its natural nuclear counterpart. It is a consequence of the two obligate genomes that OXPHOS integrity is governed by mitonuclear interactions and both genomes should match. This requires fast and efficient adaptations when variations arise in one of the genomes ${ }^{26}$. Evidence exists that the high mutation rate in the mtDNA forces counter-adaptations in the nuclear genome to restore the consequences of deleterious mtDNA mutations, as an evolutionary rescue ${ }^{27}$. Mitonuclear variation is acceptable within and between populations, most likely to allow a certain degree of adaptation to environmental changes and allows long-term sustainability of our biodiversity when environmental circumstances change, creating new selection pressures ${ }^{26}$. However, disruption of mitonuclear interactions might result in adverse health outcomes ${ }^{28}$ or, in the long term, to reproductive isolation, ultimately leading to allopatric speciation ${ }^{29}$. Evidence for an effect on fitness of mtDNA variation mainly comes from lower vertebrates ${ }^{29}$. In Drosophila melanogaster, when distinct naturally occurring mitochondrial genomes were placed against an isogenic nuclear background, gene expression was influenced by male-specific polymorphisms and led to male-specific modifications of nuclear gene expression ( 1500 genes), indicating a pervasive retrograde signalling between the genomes ${ }^{30}$. Other studies underline the role of mitonuclear interactions in determining key fitness traits and adverse health effects in other species (reviewed in ${ }^{28}$ ).

A recent review indicates that the outcomes of mitonuclear interactions are also dependent on the environment, indicating that these interactions evolve in response to natural pressures on the population ${ }^{26}$. Mitonuclear incompatibilities increase when the genetic distance becomes larger. For instance, when human cells lines are depleted of their mtDNA, repopulation with mtDNA from primates cannot restore the OXPHOS activity in these cells ${ }^{31}$. The consequences of potential disrupted mitonuclear interactions in humans are not clear. Most human populations are outbred, with considerable mixing of the genomes over recent generations ${ }^{32}$. Mitonuclear disruptions have never been described, even for couples with divergent mtDNA haplogroups, in which a potential mitonuclear mismatch is most extreme. It has been suggested that during NGT random paring is mimicked ${ }^{32}$, but long-term implications have not been studies well and generally only address the paternal mitonuclear interactions ${ }^{28}$. This is obviously a concern for NGT, as during NGT 
maternal mitonuclear interactions are disrupted and the impact of this disruption is unknown. The only experimental evidence of four healthy macaques ${ }^{19}$ born after NGT, as well as the efficient development to the blastocyst stage with no detectable effect of aneuploidy or gene expression in human embryos treated with NGT ${ }^{22}$ seems reassuring, but the consequences after implantation, until the reproductive age are not known and recent studies suggest that deleterious effects of mitonuclear incompatibilities will mainly manifest during adulthood ${ }^{28}$. Definite proof will come from the trials on NGT in humans in the U.K..

In chapter 6, we have shown that mitonuclear interactions play a role during the (mitochondrial) stress response that emerges during a Tfam knockdown, suggesting that these epistatic interactions have an important role in disease manifestation. Indeed, mitonuclear interactions are thought to be involved in the way either mtDNA mutations or nuclear genes manifest. The reduced penetrance of homoplasmic mutations in, for example, Leber's Heriditary Optic Neuropathy (LHON) and the sex differences could be explained by a role for the nuclear genome, the environment or both, although the picture is not yet clear ${ }^{26}$. In addition, mtDNA variation can also moderately affect mitochondrial function, not leading to severe mitochondrial diseases, but contributing to the severity of other genetic or non-genetic disorders, where energy metabolism is a critical factor. In genetic disorders, mtDNA variants or haplogroups may rescue or deteriorate nuclear gene mutations, thereby modifying the severity of disease expression, as has been observed in cardiomyopathies ${ }^{33}$. More study is needed to further elucidate the modifying effect of mitonuclear interactions in health and disease, which potentially adds to the complexity of mitochondrial diseases, but could also lead to disease prevention when epistatic relations can be matched ${ }^{34}$. A final observation with respect to mitonuclear interactions relates to the difference in the distribution of heteroplasmic mutations over single cells (oocytes, fibroblasts, blastomeres), which can be random or skewed (chapter 5) and which could also be the result of a nuclear factor ${ }^{35}$. Evidence exists from mice studies, in which diverse patterns of segregation are observed among tissues, with tissue-specific selection for different mtDNA genotypes ${ }^{36}$, not caused by replication efficiency or differences in OXPHOS-function ${ }^{37}$.

\section{Human mitochondrial diseases: treatment}

No effective therapy for mitochondrial disorders currently exists and several new options are currently being explored. A dysfunctional OXPHOS system is often associated with an increased production of $\mathrm{ROS}^{38}$, for which existing rescue mechanisms may fall short. In such cases, antioxidant supplementation could be used to ameliorate disease progression. Increased oxidative stress, however, appears to be mutation and patient-specific and fibroblasts of patients can be used to estimate the ROS damage and the potential benefit from antioxidant therapy ${ }^{39}$, although, at the same time, sideeffects should be carefully evaluated ${ }^{40}$. As can be learned from plants, antioxidant systems can reduce mutation loads to a lower level than in animals. Therefore, the use of a heterologous gene, encoding a supplemental oxidase (e.g. AOX), otherwise absent from mammals, has been suggested as a possibility to neutralize increased ROS production by defective OXPHOS (reviewed $\mathrm{in}^{41}$ ).

For (mitochondrial) disorders caused by nuclear genes, in some cases, 
treatments can be offered, based on the specific gene involved ${ }^{42}$. Novel approaches are being developed to correct the more challenging mtDNA mutations, but gene correction or antigenomic (e.g. transcription activator-like effector nucleases (TALEN)based technology) approaches (reviewed $\mathrm{in}^{43}$ ) to target the mtDNA particularly suffer from an evolutionary drawback. Correcting the mtDNA requires correcting a multicopy genome with a different code. Targeting the mitochondria is therefore quite cumbersome. An advantage is that it is not necessary to correct all the mtDNA copies, but it is sufficient to switch the balance to achieve a life-long effect. Promising results have been obtained in patient-derived cells, in which anti-genomic mitoTALENs were capable to specifically eliminate mutant mitochondrial genomes ${ }^{44}$. Very recently, these genome editing approaches using the mitoTALENs technology have been applied to remove mutated mtDNA from oocytes or zygotes ${ }^{45}$. Application of this approach in the NZB/BALB heteroplasmic mice, led to the selective removal of the BALB mtDNA genotype, followed by a significant decrease in the F1 offspring. Furthermore, the approach has been successfully applied to mouse MII oocytes, which were fused with human cells harboring the m.14459G>A mutation [causing Leber's hereditable optic neuropathy (LHON)] or m.9716T>C [causing neurogenic muscle weakness, ataxia and retinitis pigmentosa (NARP)]. Currently, the amount of decrease achieved with this approach is variable, and the remaining mutation load is higher as can be achieved with PGD or NGT. Although genome editing approaches can result in mutation loads below $60 \%{ }^{45}, 40 \%$ of the patients with a mutation load of $60 \%$ are for many mutations still affected ${ }^{46}$ and a more significant drop in the mutation load is required before clinical application. Furthermore, safety and efficiency need to be tested in human oocytes and zygotes. Alternative approaches that incorporate an mtDNA-encoded protein in the nuclear DNA require 1) correcting of the code ${ }^{47}, 2$ ) adding an import sequence and 3 ) having the protein imported into the mitochondria. From an evolutionary perspective, this is very unlikely to be successful, as import of these proteins has not been possible ever in eukaryotic evolution and, with only few exceptions, the 13 protein-encoding genes in the mtDNA are conserved among all species, possibly due to high hydrophobicity, as has been demonstrated ${ }^{48}$.

In this thesis (chapter 5) we have shown that healthy mitochondria can compensate dysfunctional mitochondria, thereby preventing disease manifestation. This stresses the importance of a high mtDNA copy number, as, apart from the heteroplasmic mutation level, the absolute amount of healthy mitochondria is also important. We have shown that the mtDNA copy number can differ among individual zebrafish (chapter 3 ), suggesting individual variation in the absolute amount of healthy mitochondria. Targeting this balance, e.g. through the injection of healthy mitochondria or by stimulating mitochondrial biogenesis, can be an interesting target for future therapies in order to increase the absolute amount of healthy mitochondria.

\section{Manifestation and transmission of mtDNA disorders}

\section{De novo mutations}

The interplay of a high copy mtDNA number, high (mutagenic) ROS production and replication errors create heteroplasmic mutations in somatic cells, but also in germ cells. During mitochondrial inheritance, the high copy number of the oocyte is strongly 
reduced $^{49 ;} 50$, thereby creating an increased risk for de novo mutations reaching functional significance and possible disease, when pathogenic, at the bottom of the bottleneck (Figure 7.1).

\section{Lethal or pathogenic de novo mtDNA mutations and disease}

As shown in chapter 3 for zebrafish, the mtDNA content in oocytes and the constant time-frame of preimplantation development when no mtDNA replication occurs, causes that the mtDNA copy number at the bottom of the bottleneck can be much lower than the mean of $\sim 170-200$. This correlates with the chance of a de novo mutation to become functionally manifest, as has been observed for de novo mutations that reach homoplasmy in human patients ${ }^{51-53}$. This is corroborated by our study in zebrafish oocytes (chapter 4). Large variation in the mtDNA copy number among oocytes exists in zebrafish (chapter 3), implying that all female fish possess some oocytes with a potentially high risk that de novo mutations become manifest. The mitochondrial bottleneck is highly conserved in vertebrates and considerable variation exists in the mtDNA copy number in human oocytes ${ }^{54}$, implying that a critical group of oocytes exists that have an increased risk for de novo mutations that reach high levels of heteroplasmy. This could explain the high de novo mutation rate $(\sim 20 \%)$ in human oocytes $^{55}$, as well as the high number of mtDNA disease patients $(\sim 25 \%)$ that suffer from a de novo mtDNA mutation ${ }^{11}$. The pathogenic de novo mtDNA mutations that reach high heteroplasmy levels will be subject to purifying selection (see below), and be embryonically lethal or manifest as severe, possibly fatal, disease in the offspring. As these children generally do not reproduce and the recurrence risk for the mother is very low, this will not lead to additional patients or familial disease.

\section{Escaping the mtDNA bottleneck and germline selection causes familial (de novo) disease}

As discussed in chapter 5, genetic drift and mutation-specific selection occur during maternal transmission and their interplay determines the (distribution of) mutation load(s) in the offspring. Selection occurs either on the ATP generating capacity of mitochondria or on the mitochondrial membrane potential (MMP) and only mutations negatively affecting these parameters will be selected against (chapter 5). As a result, some pathogenic mtDNA mutations are not (or to a lesser extend) subject to selection. This can either be due to the nature of the mutation, as some mutations can escape the selective mechanisms, or due to a low heteroplasmy percentage, at which the biochemical effect is compensated by the wild-type mtDNA molecules. In some particular cases, as reported for the m.8993T $>\mathrm{G}$ mutation in chapter 5, mutations leading to an increased MMP will be (undesirably) selected for, leading to a skewed segregation. The segregation patterns of additional mutations should be tested in relation to whether these mutations affect the ATP generating capacity or the MMP.

Selection during transmission has also been described in mice ${ }^{56}$. Sequencing the mtDNA of offspring from genetically modified mice harbouring multiple mtDNA mutations revealed that non-synonymous changes in protein-coding genes rapidly disappear from the genome ${ }^{56}$. Strikingly, some genes, such as ATP6, appeared to tolerate pathogenic mutations more, while other genes had a stronger signature of 
selection, such as the highly conserved COX1 and COX2 genes ${ }^{56}$, an observation that is in line with the distribution of homoplasmic mtDNA variants in humans ${ }^{57}$. Likewise, mutations were found at a much higher frequency in tRNA and rRNA genes than in protein encoding genes in both mice ${ }^{56}$ and humans ${ }^{58}$, suggesting a lower selective pressure on these genes. The distribution of tolerated functionally significant variants in genes coding for proteins is irreversibly correlated with the degree of conversation, although differences between genes exists ${ }^{57}$. In line with the m.8993T>G mutation (described in chapter 5), ATP6 mutations might not cause a significant drop in ATP production through OXPHOS and are therefore tolerated, while mutations in other OXPHOS complexes are more likely to cause drops in the ATP production and these complexes are therefore highly conserved. As a biochemical effect is minimal when heteroplasmy levels are below the level of expression, selection will not remove low-level pathogenic heteroplasmies ${ }^{59}$. This is corroborated by the high m.3243A $>$ G (in tRNA-Leu) mutation loads (>80\%) needed to cause changes in ATP generating capacity (chapter 5), which could explain the lower selection pressure on these mutations. This does not imply that tRNA mutations are moderate at the clinical level, as many of them can lead to severe and often fatal disease (Leigh syndrome; Mitochondrial Encephalomyopathy, Lactic Acidosis and Stroke-like episodes (MELAS), Myoclonic Epilepsy with Ragged Red Fibers (MERRF)). Studies in Drosophila melanogaster have suggested mutations can also escape selection as a result of co-existence of different genomes complementing each other, thereby masking a biochemical defect ${ }^{60}$. This seems unlikely for the mutations reaching a very high embryonic mutation load, as complementation would fall short in those cases.

As a result of mutations escaping selection, pathogenic mutations remain as familial disease in a population ${ }^{4}$ (Figure 7.1B) and low-frequency heteroplasmic mtDNA mutations with high pathogenic potential are present at high frequencies. The ten most prevalent mtDNA mutations have a population frequency between 1 in $155^{59}$ and 1 in $200^{61}$, indicating an even larger pool of all pathogenic mtDNA mutations within a population, which can be due to de novo mutations ${ }^{61}$ and/or less-efficient selection on transmitted mutations ${ }^{59}$. Due to genetic drift, dramatic changes in heteroplasmy levels can occur in limited numbers ${ }^{62}$ and low-level heteroplasmic mtDNA mutations can manifest in some cases in future generation as a result (if selection mechanisms are unable to purify the mutation). As the bottleneck size is highly variable (chapter 3 and 5), even within an individual ${ }^{63}$, the degree of genetic drift during inheritance varies considerably.

The combined action of the mtDNA bottleneck and selection is complex and a thorough understanding is still lacking due to experimental limitations. The combined mechanisms induce a complex mtDNA disease inheritance pattern (Figure 7.1), which is difficult to predict and might be further influenced by the nuclear background. Additional observations on mtDNA segregation will help to categorize mtDNA mutations based on their effect on OXPHOS function and MMP, which will help to predict the segregation of these mutations. 
A
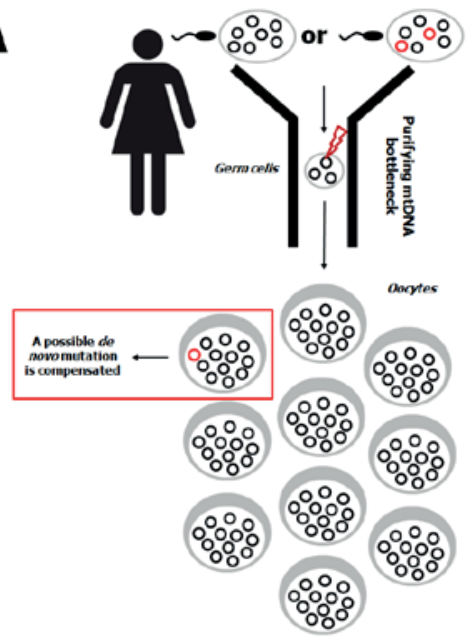

C
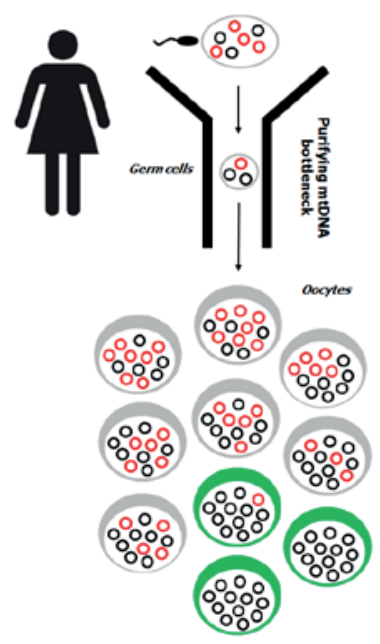

B


D
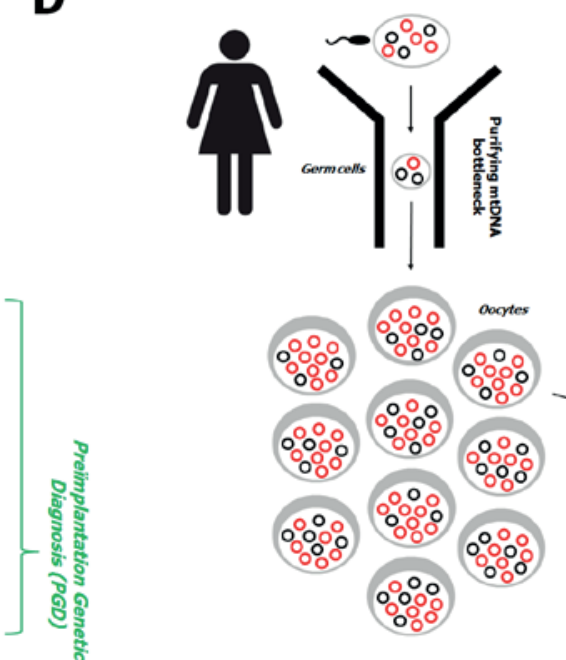

Mudear transler to motonu-
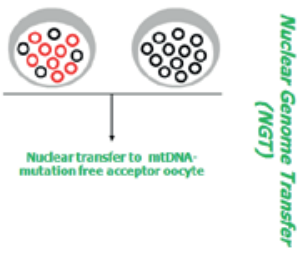

Figure 7.1 | Mitochondrial evolutionary mechanisms: implications for fertility, mtDNA disease risk and reproductive options. Mitochondrial DNA is important for healthy human reproduction (A) and altered mitochondrial parameters can cause fertility problems and mtDNA diseases (B-C). The mitochondrial evolutionary systems determine the reproductive options patients have. A) After the bottleneck, a sufficient amount of mtDNA is required for healthy reproduction. Whenever mtDNA mutations (red circles) are present, these are filtered out by the purifying bottleneck during oogenesis, rendering only oocytes without mtDNA mutations (black circles). Even if a de novo mutation occurs during the bottleneck (red thunderbolt symbol), these mutations would be compensated by the high mtDNA copy number without mutation (all below disease threshold). B) In case of a low mtDNA copy number, the risk is higher for de novo mutations to reach values above the disease threshold and affect the offspring. Usually, other oocytes are unaffected and recurrence risk is low. PND can be offered for reassurance. If the mtDNA amount in oocytes is too low, oocytes would be unable to implant (blue oocytes) or even to develop (red oocytes), according to the amount of mtDNA present. C+D) Female carriers of (familial) mtDNA mutations generally produce oocytes with different mutation loads; leading either to healthy offspring (green oocytes) with a mutation load below the level of expression or to affected offspring (grey oocytes). During preimplantation genetic diagnosis, the unaffected embryos can be selected. In some cases, all oocytes carry high mutation loads (D). In these patients, nuclear genome transfer (NGT) can be a future option, in which the nucleus of the carrier oocytes is transferred to an enucleated acceptor oocyte with healthy mtDNA molecules. 


\section{Fertility and viability}

The mtDNA copy number is important for successful fertilization and embryogenesis and the oocyte mtDNA content should be sufficient for normal vertebrate development until implantation ${ }^{64}$. The dependence of normal fertilization and reproduction on mitochondria is reflected by the large mtDNA content of fertilized oocytes compared to unfertilized oocytes ${ }^{65}$ which occurs due to clonal expansion of mtDNA during oocyte development (as described for zebrafish in chapter 3). Apparently, oocytes with an insufficient mtDNA amount are prevented from being fertilized, as indicated by the low mtDNA count in degenerate oocytes ${ }^{65}$. In mice, it was shown that oocytes with only 4,000 mtDNA copies can still be fertilized, but fail to develop after implantation and that at least 50,000 copies of mtDNA are needed in a mature oocyte to be able to resume development after implantation, which would lead to values of respectively $1-2$ and $\sim 22$ at the bottom of the bottleneck (Figure 7.1) ${ }^{6}$. Reduced fertility of oocytes with low mtDNA counts potentially limits the effect of de novo mutations, as oocytes with the highest de novo risk (those with the lowest bottleneck size) will not proceed through development.

Whether the required oocyte mtDNA levels for viable offspring are the same in humans is unclear, though a clear correlation has been observed between mtDNA content and the fertilizability of oocytes. Oocytes with fertilization failure had $\sim 152,000$ mtDNA molecules ${ }^{66}$, while in ovarian insufficiency, the count of mtDNA is $\sim 100,000^{67}$. Furthermore, based on the mtDNA mutations in chapter 5 , where $20-25 \%$ rest activity was needed to maintain OXPHOS activity in oocytes with on average $\sim 200,000 \mathrm{mtDNA}$ molecules, we estimate that, in line with mice, also $\sim 50,000$ healthy mtDNA molecules are needed for normal fertilization and early development. A negative correlation between fertilizability and mtDNA count explains the reduced fertility of oocytes from older women, since increasing maternal age reduces the mtDNA levels in oocytes ${ }^{68}$, as well as their developmental competence ${ }^{69}$. The observation that altered mtDNA copy number related to the developmental competence is corroborated by the observation that premature ovarian failure (POF) can be caused by dominant mutations in the replication domain of the POLG gene, causing mtDNA depletion ${ }^{70}$. A recent report shows that a too high mtDNA copy number at the blastocyst stage will also lead to reduced fertility, as these embryos were not be able to implant ${ }^{71}$. This suggests that compensation by replication occurs during the usually quiet preimplantation phase, probably causing a situation of stress, which is a negative factor for implantation. Together, this implies that the mtDNA copy number may be used as an independent measure for fertilization outcome ${ }^{72}$.

The role of mtDNA copy number in fertility would justify supplementation of mitochondria into infertile oocytes by injection of cytoplasm or mitochondria from donor oocytes (ooplasm) to enhance fertilization outcomes. In bovines, ooplasm transfer was able to rescue developmentally compromised oocytes (by ethidium bromide exposure) and led to proper development ${ }^{73}$. The first results in humans have been promising and suggest fertilization outcomes could indeed be improved and successful implantations were achieved, but concerns have arisen about the health consequences in the long term, as two of the 17 fetuses had an abnormal karyotype ${ }^{74}$ 
and one of the newborns was diagnosed with a sex-linked autism disorder ${ }^{75}$. Although the causality with observed chromosomal abnormalities has not been proven ${ }^{74}$, it has led to a FDA initiated stop to human ooplasm transfer. Furthermore, ooplasm transfer results in sustained, possibly detrimental, mtDNA heteroplasmy from both donor and recipient ${ }^{76}$. In pigs, mitochondial supplementation of oocytes with mtDNA deficiency enhanced embryo development and blastocyst quality and do not justify these concerns ${ }^{77}$. Nevertheless, one should be careful, especially in terms of possible mitonuclear disruptions, as described above, requiring studies on the (long-term) effects of ooplasm donation ${ }^{78}$.

Although male fertility is still ensured even when mtDNA copy numbers are low, mitochondrial function does determine spermatazoan function ${ }^{79}$. Energy for movement is thought to be mainly regulated by the mitochondrial sheath of spermatozoa ${ }^{80}$ and mitochondrial mutations can reduce sperm motility ${ }^{81}$. Although a low mtDNA content leads to low-quality sperm ${ }^{82}$, sperm with abnormal semen parameters tend to have increased mtDNA contents with lower mtDNA integrity, most likely to compensate for the defects ${ }^{83}$. Especially in those cases with asthenozoospermia (low sperm motility) caused by mitochondrial defects, intracytoplasmic sperm injection (ICSI) can be performed. During ICSI, a sperm cell is injected directly into the oocyte, and can overcome asthenozoospermic cases caused, for example, by a mitochondrial problem $^{80}$. The concern that paternal mtDNA could be sustained, whereas it is normally degraded ${ }^{84}$ has not been confirmed in ICSI follow-up studies and mice studies indicate that sperm mitochondria are also degraded after ICSI ${ }^{85 ; 86}$.

\section{Zebrafish models to study the impact and inheritance of mtDNA related disorders}

The zebrafish model offers ample opportunities to improve our knowledge of (inheritance of) mitochondrial disorders. As discussed in chapter $\mathbf{1}$ and $\mathbf{2}$, the mtDNA and its features are conserved within the animal kingdom. Consequently, the mtDNA of the zebrafish has the same complement of 37 genes and $70 \%$ similarity with its human counterpart ${ }^{87}$. Conservation of mtDNA sequence, features and the mtDNA bottleneck make the zebrafish suitable to study the impact, inheritance and treatment options of pathologies associated with mitochondrial biology.

\section{Modelling mitochondrial diseases}

Mitochondrial disorders can be caused by both mutations in the nuclear and mitochondrial DNA. For nuclear DNA mutations, genome wide diagnostic procedures, like whole-exome sequencing, reveal new potential candidate genes and mutations associated with mitochondrial disorders. In order to estimate the impact of such genes and mutations, functional studies in animal models are required that allow fast genetic modification, which are generally carried out in invertebrate models. The zebrafish model offers a unique opportunity to apply efficient invertebrate-style genetics to a vertebrate and to address vertebrate-related questions in a whole animal setting ${ }^{88}$. Both forward genetics and reverse genetics can be used in the zebrafish modele ${ }^{89}$. 
During forward genetic approaches, mutagenesis is induced using chemicals, followed by phenotypic screening and cloning of the desired gene ${ }^{89}$. If phenotypes are similar to human diseases and/or mutations are in the same genes as the nuclear genes suspected to cause mitochondrial disorders, these mutants can be further studied. Reverse genetics approaches aim at introducing mutations in selected target genes, and analyze the obtained phenotypes only afterwards. With respect to the zebrafish, a project exists in which efficient chemical mutagenesis, high-throughput sequencing and a multi-allelic phenotyping scheme is used to identify and phenotype disruptive mutations in every zebrafish protein-coding gene ${ }^{90}$. All mutant alleles and phenotypes are available online (https://www.sanger.ac.uk/resources/zebrafish/zmp/) and the mutated zebrafish lines are available from international stock centers, which enhance the use of zebrafish as a model to study genes that are suspected to have a mitochondrial function. For both of these approaches, chemical mutagenesis is mainly performed on males, thus leaving mtDNA mutations out of reach.

A more direct approach is possible by specifically engineering gene sequences. Targeted approaches to induce these mostly loss-of-function phenotypes are widespread in zebrafish. Traditionally, the function of nuclear encoded proteins during zebrafish development can be studied by transiently knocking-down the expression of these genes using antisense morpholino oligonucleotides (MOs), micro-injected in the fertilized zebrafish oocytes and active for 3-5 days ${ }^{91}$, as described in chapter 6. In this way, the zebrafish has already been used to model nuclear mitochondrial diseases, e.g. by targeting surf1 (cytochrome oxidase $c$ deficiency) ${ }^{92}$. However, concerns have arisen in using MOs in zebrafish, as in one study $80 \%$ of the morphant phenotypes appeared to be unspecific (off-target) effects, and many more specific defects were not reproduced in available mutants for the targeted gene ${ }^{93}$. A recent study suggested the existence of a compensation mechanism that would rescue mutated genes while not being effective in knock-down studies, thus giving a possible explanation for the discrepancy between knock-out and knock-down results ${ }^{94}$. In the light of this controversy, methods for rapid generation of mutants are required. Definitive reverse genetic approaches in zebrafish have become available, including zinc-finger nucleases (TALEN) ${ }^{95}$, transcription activator-like effector endonucleases ${ }^{96}$ and the clustered, regularly interspaced, short palindromic repeats (CRISPR)associated 9 (Cas9) systems ${ }^{97}$. Especially, the latter system allows efficient and multiplexed site selective, RNA-guided genome editing ${ }^{97}$. Very recently, an RNAbased method, CRISPR interference (CRISPRi) has been described for targeted silencing of transcription using a modification of the CRISPR-Cas 9 system. This system supplies a simplified approach for gene knockdown within 1-2 weeks and can be used in a variety of organisms, including zebrafish ${ }^{94}$, to study gene functions and genetic interactions ${ }^{98}$. All these approaches can be utilized to study candidate genes and mutations in the nuclear DNA for mitochondrial disorders, in order to study the impact of these mutations.

Diseases caused by mutations in the mtDNA are more complex to model, as heteroplasmy exists and mutations only exert their deleterious effects above a certain threshold. Introducing a mutation in the mtDNA requires effectively introducing a mutation in a multicopy genome. Currently, the zebrafish has not been used to model the effects of mtDNA mutations, but as mitochondria-targeted TALEN (mitoTALEN) expression have been used to eliminate mutant mitochondrial genomes in patient derived cells $^{44}$, the opposite, during which TALENs and/or CRISPRs/Cas9 are 
targeted to mitochondria in order to induce mutations in a significant proportion of the mtDNA genomes, should be possible. If such approaches result in different mutation loads, the specific effects of the mutation load can be studied in more detail, enforcing phenotype-genotype relations for mtDNA disorders.

As the variability of mitochondrial disorders is not only genetic, but also phenotypic, multiple phenotypic assays need to be present to assess the effect of mutations (both in the nuclear and in the mitochondrial genome) in full detail. The transparency of the zebrafish during development greatly improves its imaging options. Useful in this regard are the zebrafish transgenic lines enabling live imaging of the mitochondrial network and disruptions to it ${ }^{99}$. Many of such transgenic zebrafish exist. Furthermore, several phenotypes resulting from genetic defects in proteins important for mitochondrial biology have been described ${ }^{89}$, including elevated ROS production, OXPHOS inhibition (complex I activity), mitochondrial movement disruption, neuronal cell loss and behavioral abnormalities (reviewed $\mathrm{in}^{89}$ ). As mitochondrial disorders are often associated with fatigue and exercise intolerance, these behavioral measurements are important. In this regard, we also have worked on setting-up a device to measure this exercise intolerance in zebrafish, by monitoring their capacity to swim against water currents. We are currently developing assays in more detail.

No effective therapy for mitochondrial disorders currently exists. As zebrafish develop ex utero in the water, screening of novel compounds can be effectively performed in zebrafish ${ }^{100}$. For mitochondrial disorders compounds that could restore OXPHOS function exist, e.g. riboflavin ${ }^{101}$ as well as a wide variety of antioxidants ${ }^{39}$, and can be tested high-throughput in the zebrafish model. Furthermore, antigenomic strategies can be tested in the zebrafish model, both for nuclear and mitochondrial (e.g. mitoTALENs) genome defects.

\section{Unraveling the processes underlying mtDNA inheritance}

The transmission of mitochondrial DNA occurs through a genetic mtDNA bottleneck ${ }^{102}$. In chapter 3 and 4 we showed that the zebrafish model can be used to assess some basic parameters of the mtDNA bottleneck, such as size and timing, including de novo mtDNA mutation risks. Primordial germ cells (PGCs), as well as oocytes from different developmental stages ${ }^{103}$, can be isolated in high amounts from zebrafish (chapter 3).

In chapter 5 we described that genetic drift and selection underlie the inheritance of mtDNA mutations. Positive selection on wild-type mitochondria for a high, and healthy mitochondrial membrane potential (MMP) occurs during the formation of the Balbiani bodies $(\mathrm{Bb})^{104}$, a transient collection of organelles, including mitochondria, around the nucleus of primary oocytes ${ }^{105}$. Proof for the selective accumulation of mitochondria with a higher MMP in the Bb comes from zebrafish studies $^{106}$. Many studies on the formation of these evolutionary conserved structures have been performed ${ }^{107}$, indicating that studies on the formation of Bbs and the role of the MMP in the accumulation of highly active mitochondria can be further pursued in zebrafish. The localisation of active mitochondria to Balbiani bodies can be followed using fluorescent compounds, such as TMRE, a compound that concentrates inside mitochondria with a high $\mathrm{MMP}^{108}$. If a zebrafish model harbouring mtDNA mutations (such as the m.3243A>G and m.8993T>G) could be established, differences in MMP 
in oocytes of these zebrafish and wild-type oocytes can be assessed, as well as whether this relates to their accumulation in the Bb's in a large amount of samples.

Another approach to study the segregation of mtDNA in zebrafish is by making use of natural variation in the mtDNA genome between different laboratory strains ${ }^{109}$. Segregation of these neutral variants can be followed over several generations, which occurs relatively fast in zebrafish, after injecting the mtDNA of one laboratory strain inside the oocytes from a different strain. This allows a detailed evaluation of the contribution of genetic drift to the inheritance pattern of mtDNA, as has been performed in mice ${ }^{110}$, but within a large amount of samples, from PGCs to whole embryos, and to relate this to the mtDNA bottleneck size.

\section{The relation between mtDNA copy number and fertility}

Extensive research on reproductive mechanisms in teleost, which includes the zebrafish, has led to an understanding of reproduction in both fish and vertebrates as a whole ${ }^{111}$. However, compared to mammals, reproduction in fish presents several unique features ${ }^{111}$, which should be taken into account when translating reproductive research findings in fish to humans. Most of these features are the result of external fertilization and external embryogenesis in zebrafish. In contrast to most mammals, zebrafish constantly renew the oocyte stocks, forcing continuous proliferation of oogonia ${ }^{112}$. Zebrafish oocyte development has been described extensively, which includes the unique process of vitellogenesis, during which storage of yolk occurs ${ }^{103}$. The process of vitellogenesis requires an important metabolic effort ${ }^{111}$, which could be a reason for the observed differences in the oocyte mtDNA copy number between zebrafish and most mammals (chapter 3). Compared to mammals, zebrafish oocytes have no antrum ${ }^{103}$. After vitellogenesis, oocyte maturation occurs, which involves many cellular processes to enable meiosis resumption and the second metaphase ${ }^{103}$. During ovulation, oocytes are released in the ovarian cavity, followed by spawning: the deposition of eggs in the water and external fertilization. In humans, blastocysts are formed at 5 days post-fertilization, which comprises 200-300 cells, which will implant into the uterine wall, where it undergoes the process of gastrulation. Zebrafish embryos develop externally and do not implant, however, the onset of gastrulation is defined by the beginning of involution at 30-50\% epiboly, after which the germ ring appears that will give rise to two germ layers ${ }^{113}$. In humans, the blastocyst stage is the endpoint of (in vitro) preimplantation development and the zebrafish equivalent at $30-50 \%$ epiboly could serve as a similar stage in zebrafish.

A role for the mtDNA copy number in fertility has been established in mice and suggests sufficient mtDNA copies are needed in order to produce ATP to support healthy fertilization ${ }^{6}$. As shown in chapter 3, the variation in the mtDNA copy number in zebrafish oocytes within an individual is considerably large and it is likely some of these oocytes will not be fertilizable. Observations from the laboratory indeed suggest that a part of oocytes $(5-10 \%)$ is non-fertilizable; collection of these oocytes might reveal a relation to the mtDNA copy number. Alternatively, the mtDNA copy number can be reduced by targeting genes important for mtDNA replication, such as the knockdown of Tfam using MOs used in chapter 6. As the PGC pool is established in the time frame of early development, blocking of Tfam could result in a poor deposition of the PGC pool. As these PGCs will develop into oocytes, these PGCs 
might result in oocytes which cannot be fertilized, as the mtDNA copy number in these oocytes will be insufficient. In this way the zebrafish model can be used to establish firm correlations between the mtDNA copy number and fertility and viability. Injection of cytoplasm or mitochondria from donor oocytes (ooplasm) to enhance fertilization outcomes has been performed. The optimization of these techniques could also be established in the zebrafish model, as large amounts of samples enables thorough evaluation of health effects associated with such techniques.

\section{Concluding remarks and future perspectives}

The mitochondria with their own genome were indispensable for the success of eukaryotic development and the existence of complex, multicellular organisms, such as in the end human beings. However, every success has a price and in individual human beings, this price consists of mitochondrial and mtDNA-related diseases orders, infertility and (accelerated) ageing-related diseases. The consequences of mitochondrial evolution on fertility, reproduction and offspring are summarized in Figure 7.1. The studies described in this thesis contribute significantly to identify and understand the underlying mechanisms, which is of key importance for:

- designing successful gene replacement or antigenomic strategies to correct mtDNA defects;

- identifying the risk of women having children with mtDNA diseases,

- exploring the safety of innovative (reproductive) treatments, such as nuclear genome transfer, to prevent transmission of mtDNA diseases;

- options to treat mtDNA related female infertility;

- preventing mitochondrial disease by investing in energy capacity and ameliorating mitochondrial disease manifestation and progress by ROS protection.

The evolutionary conserved nature of mtDNA-related mechanisms such as the bottleneck, paves the way of studying the underlying mechanisms in animal models. As the zebrafish model combines invertebrate genetic engineering advantages with vertebrate physiology in a high number of samples, zebrafish can play a crucial role in such studies. Zebrafish can be used to:

- unravel the underlying mechanisms in the inheritance of mtDNA mutation through the bottleneck;

- study the impact of nDNAand mtDNA mutations in the development of mitochondrial disorders;

- identify and test possible therapeutic compounds to cure or ameliorate mitochondrial disorders.

Theodosius Dobzhansky stated that "Nothing in biology makes sense except in the light of evolution" and this seems particularly the case for genetics and reproduction. 


\section{References}

1. Wallace, D.C. (2007). Why do we still have a maternally inherited mitochondrial DNA? Insights from evolutionary medicine. Annual review of biochemistry 76, 781-821.

2. Bergstrom, C.T., and Pritchard, J. (1998). Germline bottlenecks and the evolutionary maintenance of mitochondrial genomes. Genetics 149, 2135-2146.

3. Shoubridge, E.A. (2001). Nuclear genetic defects of oxidative phosphorylation. Human molecular genetics 10, 2277-2284.

4. Taylor, R.W., and Turnbull, D.M. (2005). Mitochondrial DNA mutations in human disease. Nature reviews Genetics 6, 389-402.

5. Zeviani, M., and Di Donato, S. (2004). Mitochondrial disorders. Brain : a journal of neurology 127, 2153-2172.

6. Wai, T., Ao, A., Zhang, X., Cyr, D., Dufort, D., and Shoubridge, E.A. (2010). The role of mitochondrial DNA copy number in mammalian fertility. Biology of reproduction 83, 52-62.

7. de Grey, A.D. (2005). Forces maintaining organellar genomes: is any as strong as genetic code disparity or hydrophobicity? BioEssays : news and reviews in molecular, cellular and developmental biology 27, 436-446.

8. Gorman, G.S., Schaefer, A.M., Ng, Y., Gomez, N., Blakely, E.L., Alston, C.L., Feeney, C., Horvath, R., Yu-Wai-Man, P., Chinnery, P.F., et al. (2015). Prevalence of nuclear and mitochondrial DNA mutations related to adult mitochondrial disease. Annals of neurology 77 , 753-759.

9. Hellebrekers, D.M., Wolfe, R., Hendrickx, A.T., de Coo, I.F., de Die, C.E., Geraedts, J.P., Chinnery, P.F., and Smeets, H.J. (2012). PGD and heteroplasmic mitochondrial DNA point mutations: a systematic review estimating the chance of healthy offspring. Human reproduction update $18,341-349$.

10. Vento, J.M., and Pappa, B. (2013). Genetic counseling in mitochondrial disease. Neurotherapeutics : the journal of the American Society for Experimental NeuroTherapeutics 10, 243-250.

11. Sallevelt, S.C., de Die-Smulders, C.E., Hendrickx, A.T., Hellebrekers, D.M., de Coo, I.F., Alston, C.L., Knowles, C., Taylor, R.W., McFarland, R., and Smeets, H.J. (2016). De novo mtDNA point mutations are common and have a low recurrence risk. In preperation.

12. Poulton, J., and Turnbull, D.M. (2000). 74th ENMC international workshop: mitochondrial diseases 19-20 november 1999, Naarden, the netherlands. Neuromuscular disorders : NMD $10,460-462$.

13. Smeets, H.J. (2013). Preventing the transmission of mitochondrial DNA disorders: selecting the good guys or kicking out the bad guys. Reproductive biomedicine online 27, 599-610.

14. Sallevelt, S.C., Dreesen, J.C., Drusedau, M., Spierts, S., Coonen, E., van Tienen, F.H., van Golde, R.J., de Coo, I.F., Geraedts, J.P., de Die-Smulders, C.E., et al. (2013). Preimplantation genetic diagnosis in mitochondrial DNA disorders: challenge and success. Journal of medical genetics 50, 125-132.

15. Craven, L., Tuppen, H.A., Greggains, G.D., Harbottle, S.J., Murphy, J.L., Cree, L.M., Murdoch, A.P., Chinnery, P.F., Taylor, R.W., Lightowlers, R.N., et al. (2010). Pronuclear transfer in human embryos to prevent transmission of mitochondrial DNA disease. Nature 465, 82-85.

16. Sato, A., Kono, T., Nakada, K., Ishikawa, K., Inoue, S., Yonekawa, H., and Hayashi, J. (2005). Gene therapy for progeny of mito-mice carrying pathogenic mtDNA by nuclear transplantation. Proceedings of the National Academy of Sciences of the United States of America 102, 16765-16770.

17. Wang, T., Sha, H., Ji, D., Zhang, H.L., Chen, D., Cao, Y., and Zhu, J. (2014). Polar body genome transfer for preventing the transmission of inherited mitochondrial diseases. Cell 157, 1591-1604.

18. Tachibana, M., Sparman, M., Sritanaudomchai, H., Ma, H., Clepper, L., Woodward, J., Li, Y., Ramsey, C., Kolotushkina, O., and Mitalipov, S. (2009). Mitochondrial gene replacement in primate offspring and embryonic stem cells. Nature 461, 367-372.

19. Tachibana, M., Amato, P., Sparman, M., Woodward, J., Sanchis, D.M., Ma, H., Gutierrez, N.M., Tippner-Hedges, R., Kang, E., Lee, H.S., et al. (2013). Towards germline gene therapy of inherited mitochondrial diseases. Nature 493, 627-631.

20. (2011). Human Fertilisation \& Embryology Authority. Review of scientific methods to avoid mitochondrial disease. In. (

21. (2012). Nuffield Council on Bioethics. 2012. Novel techniques for the prevention of 
22. Hyslop, L.A., Blakeley, P., Craven, L., Richardson, J., Fogarty, N.M., Fragouli, E., Lamb, M., Wamaitha, S.E., Prathalingam, N., Zhang, Q., et al. (2016). Towards clinical application of pronuclear transfer to prevent mitochondrial DNA disease. Nature 534, 383-386.

23. Poulton, J., and Oakeshott, P. (2012). Nuclear transfer to prevent maternal transmission of mitochondrial DNA disease. Bmj 345, e6651.

24. Yamada, M., Emmanuele, V., Sanchez-Quintero, M.J., Sun, B., Lallos, G., Paull, D., Zimmer, M., Pagett, S., Prosser, R.W., Sauer, M.V., et al. (2016). Genetic Drift Can Compromise Mitochondrial Replacement by Nuclear Transfer in Human Oocytes. Cell Stem Cell.

25. Sharpley, M.S., Marciniak, C., Eckel-Mahan, K., McManus, M., Crimi, M., Waymire, K., Lin, C.S., Masubuchi, S., Friend, N., Koike, M., et al. (2012). Heteroplasmy of mouse mtDNA is genetically unstable and results in altered behavior and cognition. Cell 151, 333-343.

26. Wolff, J.N., Ladoukakis, E.D., Enriquez, J.A., and Dowling, D.K. (2014). Mitonuclear interactions: evolutionary consequences over multiple biological scales. Philosophical transactions of the Royal Society of London Series B, Biological sciences 369, 20130443.

27. Dowling, D.K., Friberg, U., and Lindell, J. (2008). Evolutionary implications of non-neutral mitochondrial genetic variation. Trends in ecology \& evolution 23, 546-554.

28. Reinhardt, K., Dowling, D.K., and Morrow, E.H. (2013). Medicine. Mitochondrial replacement, evolution, and the clinic. Science 341, 1345-1346.

29. Barreto, F.S., and Burton, R.S. (2013). Evidence for compensatory evolution of ribosomal proteins in response to rapid divergence of mitochondrial rRNA. Molecular biology and evolution 30, 310-314.

30. Innocenti, P., Morrow, E.H., and Dowling, D.K. (2011). Experimental evidence supports a sexspecific selective sieve in mitochondrial genome evolution. Science 332, 845-848.

31. Kenyon, L., and Moraes, C.T. (1997). Expanding the functional human mitochondrial DNA database by the establishment of primate xenomitochondrial cybrids. Proceedings of the National Academy of Sciences of the United States of America 94, 9131-9135.

32. Chinnery, P.F., Craven, L., Mitalipov, S., Stewart, J.B., Herbert, M., and Turnbull, D.M. (2014). The challenges of mitochondrial replacement. PLoS genetics 10, e1004315.

33. Strauss, K.A., DuBiner, L., Simon, M., Zaragoza, M., Sengupta, P.P., Li, P., Narula, N., Dreike, S., Platt, J., Procaccio, V., et al. (2013). Severity of cardiomyopathy associated with adenine nucleotide translocator-1 deficiency correlates with mtDNA haplogroup. Proceedings of the National Academy of Sciences of the United States of America 110, 3453-3458.

34. Moreno-Loshuertos, R., Ferrin, G., Acin-Perez, R., Gallardo, M.E., Viscomi, C., Perez-Martos, A., Zeviani, M., Fernandez-Silva, P., and Enriquez, J.A. (2011). Evolution meets disease: penetrance and functional epistasis of mitochondrial tRNA mutations. PLoS genetics 7, e1001379.

35. Blok, R.B., Gook, D.A., Thorburn, D.R., and Dahl, H.H. (1997). Skewed segregation of the mtDNA nt 8993 (T-->G) mutation in human oocytes. American journal of human genetics 60 , 1495-1501.

36. Jenuth, J.P., Peterson, A.C., and Shoubridge, E.A. (1997). Tissue-specific selection for different mtDNA genotypes in heteroplasmic mice. Nature genetics 16, 93-95.

37. Battersby, B.J., and Shoubridge, E.A. (2001). Selection of a mtDNA sequence variant in hepatocytes of heteroplasmic mice is not due to differences in respiratory chain function or efficiency of replication. Human molecular genetics 10, 2469-2479.

38. Vives-Bauza, C., Gonzalo, R., Manfredi, G., Garcia-Arumi, E., and Andreu, A.L. (2006). Enhanced ROS production and antioxidant defenses in cybrids harbouring mutations in mtDNA. Neuroscience letters 391, 136-141.

39. Voets, A.M., Lindsey, P.J., Vanherle, S.J., Timmer, E.D., Esseling, J.J., Koopman, W.J., Willems, P.H., Schoonderwoerd, G.C., De Groote, D., Poll-The, B.T., et al. (2012). Patientderived fibroblasts indicate oxidative stress status and may justify antioxidant therapy in OXPHOS disorders. Biochimica et biophysica acta 1817, 1971-1978.

40. Bjelakovic, G., Nikolova, D., Gluud, L.L., Simonetti, R.G., and Gluud, C. (2012). Antioxidant supplements for prevention of mortality in healthy participants and patients with various diseases. The Cochrane database of systematic reviews 3, CD007176.

41. El-Khoury, R., Kemppainen, K.K., Dufour, E., Szibor, M., Jacobs, H.T., and Rustin, P. (2014). Engineering the alternative oxidase gene to better understand and counteract mitochondrial defects: state of the art and perspectives. British journal of pharmacology 171, 2243-2249.

42. Gerards, M. (2014). Leigh syndrome: the genetic heterogeneity story continues. Brain : a journal of neurology 137, 2872-2873.

43. Russell, O., and Turnbull, D. (2014). Mitochondrial DNA disease-molecular insights and 
potential routes to a cure. Experimental cell research 325, 38-43.

44. Bacman, S.R., Williams, S.L., Pinto, M., Peralta, S., and Moraes, C.T. (2013). Specific elimination of mutant mitochondrial genomes in patient-derived cells by mitoTALENs. Nature medicine 19, 1111-1113.

45. Reddy, P., Ocampo, A., Suzuki, K., Luo, J., Bacman, S.R., Williams, S.L., Sugawara, A., Okamura, D., Tsunekawa, Y., Wu, J., et al. (2015). Selective elimination of mitochondrial mutations in the germline by genome editing. Cell 161, 459-469.

46. Smeets, H.J., Sallevelt, S.C., Dreesen, J.C., de Die-Smulders, C.E., and de Coo, I.F. (2015). Preventing the transmission of mitochondrial DNA disorders using prenatal or preimplantation genetic diagnosis. Annals of the New York Academy of Sciences 1350, 29-36.

47. Nagley, P., Farrell, L.B., Gearing, D.P., Nero, D., Meltzer, S., and Devenish, R.J. (1988). Assembly of functional proton-translocating ATPase complex in yeast mitochondria with cytoplasmically synthesized subunit 8 , a polypeptide normally encoded within the organelle. Proceedings of the National Academy of Sciences of the United States of America 85, 20912095.

48. Perales-Clemente, E., Fernandez-Silva, P., Acin-Perez, R., Perez-Martos, A., and Enriquez, J.A. (2011). Allotopic expression of mitochondrial-encoded genes in mammals: achieved goal, undemonstrated mechanism or impossible task? Nucleic acids research 39, 225-234.

49. Cree, L.M., Samuels, D.C., de Sousa Lopes, S.C., Rajasimha, H.K., Wonnapinij, P., Mann, J.R., Dahl, H.H., and Chinnery, P.F. (2008). A reduction of mitochondrial DNA molecules during embryogenesis explains the rapid segregation of genotypes. Nature genetics 40, 249254.

50. Wai, T., Teoli, D., and Shoubridge, E.A. (2008). The mitochondrial DNA genetic bottleneck results from replication of a subpopulation of genomes. Nature genetics 40, 1484-1488.

51. Burrage, L.C., Tang, S., Wang, J., Donti, T.R., Walkiewicz, M., Luchak, J.M., Chen, L.C., Schmitt, E.S., Niu, Z., Erana, R., et al. (2014). Mitochondrial myopathy, lactic acidosis, and sideroblastic anemia (MLASA) plus associated with a novel de novo mutation $(\mathrm{m} .8969 \mathrm{G}>\mathrm{A})$ in the mitochondrial encoded ATP6 gene. Molecular genetics and metabolism.

52. Degoul, F., Francois, D., Diry, M., Ponsot, G., Desguerre, I., Heron, B., Marsac, C., and Moutard, M.L. (1997). A near homoplasmic T8993G mtDNA mutation in a patient with atypic Leigh syndrome not present in the mother's tissues. Journal of inherited metabolic disease 20, 49-53.

53. Maassen, J.A., Biberoglu, S., t Hart, L.M., Bakker, E., and de Knijff, P. (2002). A case of a de novo A3243G mutation in mitochondrial DNA in a patient with diabetes and deafness. Archives of physiology and biochemistry 110, 186-188.

54. Otten, A.B., and Smeets, H.J. (2015). Evolutionary defined role of the mitochondrial DNA in fertility, disease and ageing. Human reproduction update.

55. Jacobs, L., Gerards, M., Chinnery, P., Dumoulin, J., de Coo, I., Geraedts, J., and Smeets, H. (2007). mtDNA point mutations are present at various levels of heteroplasmy in human oocytes. Molecular human reproduction 13, 149-154.

56. Stewart, J.B., Freyer, C., Elson, J.L., Wredenberg, A., Cansu, Z., Trifunovic, A., and Larsson, N.G. (2008). Strong purifying selection in transmission of mammalian mitochondrial DNA. PLoS biology 6, e10.

57. Voets, A.M., van den Bosch, B.J., Stassen, A.P., Hendrickx, A.T., Hellebrekers, D.M., Van Laer, L., Van Eyken, E., Van Camp, G., Pyle, A., Baudouin, S.V., et al. (2011). Large scale mtDNA sequencing reveals sequence and functional conservation as major determinants of homoplasmic mtDNA variant distribution. Mitochondrion 11, 964-972.

58. Ruiz-Pesini, E., and Wallace, D.C. (2006). Evidence for adaptive selection acting on the tRNA and rRNA genes of human mitochondrial DNA. Human mutation 27, 1072-1081.

59. Ye, K., Lu, J., Ma, F., Keinan, A., and Gu, Z. (2014). Extensive pathogenicity of mitochondrial heteroplasmy in healthy human individuals. Proceedings of the National Academy of Sciences of the United States of America 111, 10654-10659.

60. Ma, H., Xu, H., and O'Farrell, P.H. (2014). Transmission of mitochondrial mutations and action of purifying selection in Drosophila melanogaster. Nature genetics 46, 393-397.

61. Elliott, H.R., Samuels, D.C., Eden, J.A., Relton, C.L., and Chinnery, P.F. (2008). Pathogenic mitochondrial DNA mutations are common in the general population. American journal of human genetics 83, 254-260.

62. Brown, D.T., Samuels, D.C., Michael, E.M., Turnbull, D.M., and Chinnery, P.F. (2001). Random genetic drift determines the level of mutant mtDNA in human primary oocytes. American journal of human genetics 68, 533-536.

63. Wilson, I.J., Carling, P.J., Alston, C.L., Floros, V.I., Pyle, A., Hudson, G., Sallevelt, 
S.C., Lamperti, C., Carelli, V., Bindoff, L.A., et al. (2016). Mitochondrial DNA sequence characteristics modulate the size of the genetic bottleneck. Human molecular genetics.

64. Ebert, K.M., Liem, H., and Hecht, N.B. (1988). Mitochondrial DNA in the mouse preimplantation embryo. Journal of reproduction and fertility 82, 145-149.

65. Santos, T.A., El Shourbagy, S., and St John, J.C. (2006). Mitochondrial content reflects oocyte variability and fertilization outcome. Fertility and sterility $85,584-591$.

66. Reynier, P., May-Panloup, P., Chretien, M.F., Morgan, C.J., Jean, M., Savagner, F., Barriere, P., and Malthiery, Y. (2001). Mitochondrial DNA content affects the fertilizability of human oocytes. Molecular human reproduction 7, 425-429.

67. May-Panloup, P., Chretien, M.F., Jacques, C., Vasseur, C., Malthiery, Y., and Reynier, P. (2005). Low oocyte mitochondrial DNA content in ovarian insufficiency. Human reproduction 20, 593-597.

68. de Boer, K., Janssen, R., Leigh, D., and Mortimer, D. (1999). O-165 quantification of mtDNA copy number in the human secondary oocyte. Human reproduction 14, 2419.

69. Yamamoto, T., Iwata, H., Goto, H., Shiratuki, S., Tanaka, H., Monji, Y., and Kuwayama, T. (2010). Effect of maternal age on the developmental competence and progression of nuclear maturation in bovine oocytes. Molecular reproduction and development 77, 595-604.

70. Pagnamenta, A.T., Taanman, J.W., Wilson, C.J., Anderson, N.E., Marotta, R., Duncan, A.J., Bitner-Glindzicz, M., Taylor, R.W., Laskowski, A., Thorburn, D.R., et al. (2006). Dominant inheritance of premature ovarian failure associated with mutant mitochondrial DNA polymerase gamma. Human reproduction 21, 2467-2473.

71. Fragouli, E., Spath, K., Alfarawati, S., Kaper, F., Craig, A., Michel, C.E., Kokocinski, F., Cohen, J., Munne, S., and Wells, D. (2015). Altered levels of mitochondrial DNA are associated with female age, aneuploidy, and provide an independent measure of embryonic implantation potential. PLoS genetics 11, e1005241.

72. Seli, E. (2016). Mitochondrial DNA as a biomarker for in-vitro fertilization outcome. Curr Opin Obstet Gynecol 28, 158-163.

73. Chiaratti, M.R., Ferreira, C.R., Perecin, F., Meo, S.C., Sangalli, J.R., Mesquita, L.G., de Carvalho Balieiro, J.C., Smith, L.C., Garcia, J.M., and Meirelles, F.V. (2011). Ooplastmediated developmental rescue of bovine oocytes exposed to ethidium bromide. Reproductive biomedicine online 22, 172-183.

74. Barritt, J.A., Brenner, C.A., Malter, H.E., and Cohen, J. (2001). Mitochondria in human offspring derived from ooplasmic transplantation. Human reproduction 16, 513-516.

75. Levy, R., Elder, K., and Menezo, Y. (2004). Cytoplasmic transfer in oocytes: biochemical aspects. Human reproduction update 10, 241-250.

76. Brenner, C.A., Barritt, J.A., Willadsen, S., and Cohen, J. (2000). Mitochondrial DNA heteroplasmy after human ooplasmic transplantation. Fertility and sterility 74, 573-578.

77. Cagnone, G.L., Tsai, T.S., Makanji, Y., Matthews, P., Gould, J., Bonkowski, M.S., Elgass, K.D., Wong, A.S., Wu, L.E., McKenzie, M., et al. (2016). Restoration of normal embryogenesis by mitochondrial supplementation in pig oocytes exhibiting mitochondrial DNA deficiency. Sci Rep 6, 23229.

78. St John, J.C. (2002). Ooplasm donation in humans: the need to investigate the transmission of mitochondrial DNA following cytoplasmic transfer. Human reproduction 17, 1954-1958.

79. Ruiz-Pesini, E., Lapena, A.C., Diez, C., Alvarez, E., Enriquez, J.A., and Lopez-Perez, M.J. (2000). Seminal quality correlates with mitochondrial functionality. Clinica chimica acta; international journal of clinical chemistry 300, 97-105.

80. Rawe, V.Y., Hermes, R., Nodar, F.N., Fiszbajn, G., and Chemes, H.E. (2007). Results of intracytoplasmic sperm injection in two infertile patients with abnormal organization of sperm mitochondrial sheaths and severe asthenoteratozoospermia. Fertility and sterility 88, 649-653.

81. Holyoake, A.J., McHugh, P., Wu, M., O'Carroll, S., Benny, P., Sin, I.L., and Sin, F.Y. (2001). High incidence of single nucleotide substitutions in the mitochondrial genome is associated with poor semen parameters in men. International journal of andrology 24, 175-182.

82. Amaral, A., Lourenco, B., Marques, M., and Ramalho-Santos, J. (2013). Mitochondria functionality and sperm quality. Reproduction 146, R163-174.

83. Song, G.J., and Lewis, V. (2008). Mitochondrial DNA integrity and copy number in sperm from infertile men. Fertility and sterility 90, 2238-2244.

84. St John, J., Sakkas, D., Dimitriadi, K., Barnes, A., Maclin, V., Ramey, J., Barratt, C., and De Jonge, C. (2000). Failure of elimination of paternal mitochondrial DNA in abnormal embryos. Lancet 355, 200.

85. Cummins, J.M., Wakayama, T., and Yanagimachi, R. (1997). Fate of microinjected sperm components in the mouse oocyte and embryo. Zygote 5, 301-308. 
86. Danan, C., Sternberg, D., Van Steirteghem, A., Cazeneuve, C., Duquesnoy, P., Besmond, C., Goossens, M., Lissens, W., and Amselem, S. (1999). Evaluation of parental mitochondrial inheritance in neonates born after intracytoplasmic sperm injection. American journal of human genetics 65 , 463-473.

87. Howe, K., Clark, M.D., Torroja, C.F., Torrance, J., Berthelot, C., Muffato, M., Collins, J.E., Humphray, S., McLaren, K., Matthews, L., et al. (2013). The zebrafish reference genome sequence and its relationship to the human genome. Nature 496, 498-503.

88. Lieschke, G.J., and Currie, P.D. (2007). Animal models of human disease: zebrafish swim into view. Nature reviews Genetics 8, 353-367.

89. Steele, S.L., Prykhozhij, S.V., and Berman, J.N. (2014). Zebrafish as a model system for mitochondrial biology and diseases. Translational research : the journal of laboratory and clinical medicine 163, 79-98.

90. Kettleborough, R.N., Busch-Nentwich, E.M., Harvey, S.A., Dooley, C.M., de Bruijn, E., van Eeden, F., Sealy, I., White, R.J., Herd, C., Nijman, I.J., et al. (2013). A systematic genomewide analysis of zebrafish protein-coding gene function. Nature 496, 494-497.

91. Nasevicius, A., and Ekker, S.C. (2000). Effective targeted gene 'knockdown' in zebrafish. Nature genetics 26, 216-220.

92. Baden, K.N., Murray, J., Capaldi, R.A., and Guillemin, K. (2007). Early developmental pathology due to cytochrome c oxidase deficiency is revealed by a new zebrafish model. The Journal of biological chemistry 282, 34839-34849.

93. Kok, F.O., Shin, M., Ni, C.W., Gupta, A., Grosse, A.S., van Impel, A., Kirchmaier, B.C., Peterson-Maduro, J., Kourkoulis, G., Male, I., et al. (2015). Reverse genetic screening reveals poor correlation between morpholino-induced and mutant phenotypes in zebrafish. Developmental cell 32, 97-108.

94. Rossi, A., Kontarakis, Z., Gerri, C., Nolte, H., Holper, S., Kruger, M., and Stainier, D.Y. (2015). Genetic compensation induced by deleterious mutations but not gene knockdowns. Nature 524, 230-233.

95. Doyon, Y., McCammon, J.M., Miller, J.C., Faraji, F., Ngo, C., Katibah, G.E., Amora, R., Hocking, T.D., Zhang, L., Rebar, E.J., et al. (2008). Heritable targeted gene disruption in zebrafish using designed zinc-finger nucleases. Nature biotechnology 26, 702-708.

96. Cade, L., Reyon, D., Hwang, W.Y., Tsai, S.Q., Patel, S., Khayter, C., Joung, J.K., Sander, J.D., Peterson, R.T., and Yeh, J.R. (2012). Highly efficient generation of heritable zebrafish gene mutations using homo- and heterodimeric TALENs. Nucleic acids research 40, 80018010.

97. Hwang, W.Y., Fu, Y., Reyon, D., Maeder, M.L., Tsai, S.Q., Sander, J.D., Peterson, R.T., Yeh, J.R., and Joung, J.K. (2013). Efficient genome editing in zebrafish using a CRISPR-Cas system. Nature biotechnology 31, 227-229.

98. Qi, L.S., Larson, M.H., Gilbert, L.A., Doudna, J.A., Weissman, J.S., Arkin, A.P., and Lim, W.A. (2013). Repurposing CRISPR as an RNA-guided platform for sequence-specific control of gene expression. Cell 152, 1173-1183.

99. Kim, M.J., Kang, K.H., Kim, C.H., and Choi, S.Y. (2008). Real-time imaging of mitochondria in transgenic zebrafish expressing mitochondrially targeted GFP. BioTechniques 45, 331-334.

100. Ridges, S., Heaton, W.L., Joshi, D., Choi, H., Eiring, A., Batchelor, L., Choudhry, P., Manos, E.J., Sofla, H., Sanati, A., et al. (2012). Zebrafish screen identifies novel compound with selective toxicity against leukemia. Blood 119, 5621-5631.

101. Gerards, M., van den Bosch, B.J., Danhauser, K., Serre, V., van Weeghel, M., Wanders, R.J., Nicolaes, G.A., Sluiter, W., Schoonderwoerd, K., Scholte, H.R., et al. (2011). Riboflavinresponsive oxidative phosphorylation complex I deficiency caused by defective ACAD9: new function for an old gene. Brain : a journal of neurology 134, 210-219.

102. Wolff, J.N., White, D.J., Woodhams, M., White, H.E., and Gemmell, N.J. (2011). The strength and timing of the mitochondrial bottleneck in salmon suggests a conserved mechanism in vertebrates. PloS one 6, e20522.

103. Selman, K., Wallace, R.A., Sarka, A., and Qi, X. (1993). Stages of oocyte development in the zebrafish, Brachydanio rerio. Journal of Morphology 218, 203-224.

104. Zhou, R.R., Wang, B., Wang, J., Schatten, H., and Zhang, Y.Z. (2010). Is the mitochondrial cloud the selection machinery for preferentially transmitting wild-type mtDNA between generations? Rewinding Muller's ratchet efficiently. Current genetics 56, 101-107.

105. Guraya, S.S. (1979). Recent advances in the morphology, cytochemistry, and function of Balbiani's vitelline body in animal oocytes. International review of cytology 59, 249-321.

106. Zhang, Y.Z., Ouyang, Y.C., Hou, Y., Schatten, H., Chen, D.Y., and Sun, Q.Y. (2008). Mitochondrial behavior during oogenesis in zebrafish: a confocal microscopy analysis. 
Development, growth \& differentiation 50, 189-201

107. Gupta, T., Marlow, F.L., Ferriola, D., Mackiewicz, K., Dapprich, J., Monos, D., and Mullins, M.C. (2010). Microtubule actin crosslinking factor 1 regulates the Balbiani body and animalvegetal polarity of the zebrafish oocyte. PLoS genetics 6, e1001073.

108. Mitra, K., and Lippincott-Schwartz, J. (2010). Analysis of mitochondrial dynamics and functions using imaging approaches. Current protocols in cell biology / editorial board, Juan S Bonifacino [et al] Chapter 4, Unit 425 21-21.

109. Flynn, T., Signal, B., Johnson, S.L., and Gemmell, N.J. (2015). Mitochondrial genome diversity among six laboratory zebrafish (Danio rerio) strains. Mitochondrial DNA, 1-8.

110. Jenuth, J.P., Peterson, A.C., Fu, K., and Shoubridge, E.A. (1996). Random genetic drift in the female germline explains the rapid segregation of mammalian mitochondrial DNA. Nature genetics 14, 146-151.

111. Jalabert, B. (2005). Particularities of reproduction and oogenesis in teleost fish compared to mammals. Reproduction, nutrition, development 45, 261-279.

112. Tokarz, R.R. (1978). Oogonial proliferation, oogenesis and folliculogenesis in nonmammalian vertebrates. In The Vertebrate Ovary - Comparative Biology and Evolution, R.E. Jones, ed. (New York, Plenum Press), pp 145-179.

113. Kimmel, C.B., Ballard, W.W., Kimmel, S.R., Ullmann, B., and Schilling, T.F. (1995). Stages of embryonic development of the zebrafish. Developmental dynamics : an official publication of the American Association of Anatomists 203, 253-310. 

Summary/Samenvatting 


\section{Summary}

Mitochondrial disorders are a group of inheritable, often fatal diseases or syndromes, which exhibit a wide variety of clinical symptoms. Mitochondrial disorders are mostly caused by an abnormality of the ATP-generating machinery, the oxidative phosphorylation (OXPHOS), and affect about 1 in 5,000 individuals. Organs with high-energy demands are more prone to OXPHOS dysfunction and brain and skeletal muscle are therefore the most severely affected tissues. Besides being clinically heterogeneous, mitochondrial disorders are also characterized by genetic heterogeneity, as similar phenotypes can be caused by mutations in different genes. In addition, the genes encoding proteins for mitochondrial function are distributed over two genomes. Part of the genes lay in the nuclear DNA, while some are part of the genetic material of the mitochondria: the mitochondrial DNA (mtDNA). The mtDNA is markedly different from its nuclear counterpart. The mtDNA is a multicopy genome and cells maintain a high mtDNA copy number. As a result, a mixture of wilt-type and variant or mutant mtDNA generally co-exists, a state referred to as heteroplasmy. A mtDNA disease only manifest if the heteroplasmy level (or mutation load) exceeds a certain threshold. Also, the mode of inheritance is different from its nuclear counterpart. While the nuclear DNA has a Mendelian inheritance pattern, the mtDNA transmits exclusively from mother to child. During its maternal transmission, the mtDNA segregates through a genetic bottleneck, which is a restriction in the number of mtDNA molecules from the oocytes being transmitted to the cells of the offspring. The mechanisms underlying this mtDNA bottleneck are still unclear.

The overall aim of this thesis is to characterize the processes that underlie mtDNA segregation through the mtDNA bottleneck and to explain the de novo mtDNA disease risks. Specific aims are:

- to define the size of the mtDNA bottleneck during transmission;

- to investigate the risk of acquiring de novo mtDNA mutations during oogenesis;

- to identify the biological processes that underlie the mtDNA bottleneck and the (mutation-specific) shifts in mtDNA heteroplasmy levels between mothers and children;

- to study the effect of a low mtDNA copy number on embryogenesis.

In chapter 2 we reviewed the evolution of mitochondria and their genomes. The existence of two different genomes can be traced back to the origin of eukaryotes, which is thought to have originated as a result of an endosymbiosis event between two prokaryotes, both possessing their own genome. During this endosymbiosis a hydrogen-dependent anaerobic archaebacterium engulfed a respiring eubacterium, which enabled the host to generate large amounts of intracellular hydrogen-dependent adenosine triphosphate (ATP). The eubacterium became the mitochondria with their own genome, the mtDNA. The downside was an increase of reactive oxygen species (ROS) trapped inside the eukaryotic cell, resulting in high mtDNA mutation rates. According to Muller's ratchet theory, accumulation of mutations in the asexually transmitted mtDNA would occur, ultimately leading to reduced reproductive fitness and eventually extinction. However, mitochondria have persisted over the course of evolution, initially due to a rapid, extreme decrease in the mtDNA content through the process of endosymbiotic gene transfer (EGT) from the mtDNA to its nuclear counterpart. After the phylogenetic divergence of eukaryotes into animals, fungi and 
plants, additional adaptations have been selected that decrease the detrimental effect of high mtDNA mutation rates, which has resulted in different DNA characteristics. Animals have compact mitochondrial genomes, with little recombination, a stable number of genes and a high mtDNA copy number, whereas plants have larger genomes with variable gene counts, a low mtDNA copy number and many recombination events. Fungal mtDNA is somewhere in between. In plants, the mtDNA mutation rate is kept low, by effective ROS defence and efficient recombination-mediated mtDNA repair. In animal mtDNA, these mechanisms are not or less-well developed and the detrimental mutagenesis events are controlled by a high mtDNA copy number in combination with a genetic bottleneck and purifying selection during transmission. Mobile animals with a high mtDNA mutation rate can survive better due to an increased capacity to adapt to more rapidly to changing environmental conditions in terms of energy production, whereas static plants do not have this need. In animals, a high mtDNA copy number and the mitochondrial genetic bottleneck are crucial for guarding the balance between positive, negative and neutral mtDNA variants, which arise as a result of the high mtDNA mutation rate. The mtDNA genetic bottleneck is highly conserved among the animal kingdom, reflecting its importance for the maintenance of a homoplasmic wildtype mtDNA genome in animals.

The mtDNA bottleneck is a restriction in the number of mtDNA molecules being transmitted from the oocyte to the offspring, thereby creating a sampling effect. Low heteroplasmic mtDNA mutations can be filtered out in this way. Currently, size, timing, variation and possible differences between germline and non-germline cells are not precisely known. In chapter $\mathbf{3}$ we used the zebrafish model to characterize the mtDNA bottleneck mechanism in full detail. Zebrafish allow easy collection of high numbers of (immature) oocytes, primordial germ cells (PGCs) and non-germline cells throughout embryonic development. Mature zebrafish oocytes harboured, on average, $\sim 19.0 \times 10^{6} \mathrm{mtDNA}$ molecules with high variation between oocytes. During the earliest stages of embryogenesis, the mtDNA copy number is divided over the daughter cells in the absence of mtDNA replication. As a result, the mtDNA copy number decreases to $\sim 170 \mathrm{mtDNA}$ molecules per PGC, a number similar to mammals, and to $\sim 50$ in the non-germline cells. As the lowest mtDNA copy number occurs at a fixed developmental stage, variation in the oocyte mtDNA copy number dictates the mtDNA amount at the bottom of the bottleneck.

To test whether a low mtDNA copy number at the bottom of the bottleneck renders a group of oocytes vulnerable for de novo mutations reaching significant heteroplasmy levels, we performed next-generation sequencing (NGS) of the mtDNA in 103 individual oocytes from eight different zebrafish (chapter 4). Statistical and biological filters were applied to reliably detect variants with heteroplasmy $\geq 1.5 \%$. Randomly scattered de novo mutations were detected in $\sim 20 \%$ of the oocytes. This is in line with our observation in chapter 3 that $\sim 20 \%$ of the germ cells have a mtDNA copy number at the bottom of the bottleneck of $\leq 73$. Mutations arising at this point lead to detectable mutation loads. As the frequency of de novo mutations is close to the reported error rate of the mitochondrial replication-enzyme polymerase gamma $(P O L G)$, this seems to be the predominant factor. Moreover, this could also explain the high frequency of de novo mutations ( 25\%) observed in human patients.

We further characterized the processes underlying the bottleneck in chapter 5 , by studying the mutation load distribution of three mtDNA mutations (m.3243A>G, m.8993T $>\mathrm{G}$ and $\mathrm{m} .14487 \mathrm{~T}>\mathrm{C}$ ) in oocytes, zygotes and embryos from multiple 
preimplantation genetic diagnosis (PGD) cycles. Assuming neutral genetic drift, effective bottleneck sizes were calculated to range from 49-152 (m.3243A>G) to 21 ( $\mathrm{m} .14487 \mathrm{~T}>\mathrm{C})$ and $10(\mathrm{~m} .8993 \mathrm{~T}>\mathrm{G})$, which is in line with our observations in chapter 3. However, a correlation between m.3243A $>G$ mutation load and the estimated bottleneck size, the absence of $m .3243 A>G$ mutation loads $>80 \%$, and a consistent skewed distribution for the m.8993T>G mutation suggested that non-random, selection events also take place during mtDNA inheritance. During oogenesis and early embryonic development cells completely rely on OXPHOS and an abolished OXPHOS (e.g. when m.3243A $>$ G loads $>80 \%$ ) makes cells unable to pass these stages. In contrast, the residual OXPHOS-activity of cells harbouring the m.8993T>G or $\mathrm{m} .14487 \mathrm{~T}>\mathrm{C}$ mutation appears sufficient, even at $\sim 100 \%$ mutation load, probably compensated by the high mitochondrial copy number. Positive selection for wellfunctioning mitochondria with a high mitochondrial membrane potential (MMP), as a result of the pumping of protons across the mitochondrial inner membrane during the process of OXPHOS, also occurs during this phase. The MMP is reduced for most mutations negatively affecting OXPHOS function. However, for the m.8993T>G mutation the MMP is higher than in mitochondria with wild-type mtDNA, resulting in positive selection for mitochondria with this mutation. The absence of a clear OXPHOS or MMP defect associated with the m.14487T $>C$ mutation causes a distribution by neutral genetic drift only. mtDNA inheritance is thus defined by the interplay of genetic drift and mutation-specific selection events. This should be taken into account, when applying prevention strategies, like PGD and nuclear genome transfer (NGT), as this affects the mutation load that is considered to be safe for transferring a healthy embryo.

As described in chapter 3 , the mtDNA copy number is extensively replicated during oogenesis to achieve a high mtDNA copy number. This is essential for reproduction, as the mtDNA copy number correlates with both the fertility and viability of oocytes and early embryos. In chapter $\mathbf{6}$, we investigated the effect of a reduced mtDNA copy number during early development by knocking down mitochondrial transcription factor A (Tfam), a key regulator of the mtDNA copy number, using morpholino oligonucleotides (MOs). Embryos with a Tfam knockdown displayed an embryonic developmental defect, especially of the heart and the brain. The effect was most predominant at 4 days post fertilization (dpf). These embryos had a $50-80 \%$ decrease in the mtDNA copy number compared to control-injected embryos. Using transcriptomics, we investigated the effect of Tfam knockdown on the expression of other genes and pathways at $4 \mathrm{dpf}$. Morphant zebrafish displayed downregulation of mtDNA-encoded proteins involved in the electron transport chain. Of the nuclear-encoded pathways, metabolic pathways as well as pathways important for proliferation during embryogenesis were downregulated, while we observed a significant upregulation of pathways involved in cell cycle and cell signalling pathways important for embryogenesis, and of the mitochondrial translation machinery. In line with the upregulation of the Fas pathway (stress induction of heat shock proteins), we also observed an upregulation of specific genes involved in the mitochondrial unfolded protein response, some mitochondrial matrix protease genes, as well as some key genes in apoptosis. Based on these data, we conclude that maintaining and increasing the mtDNA copy number is crucial for normal embryonic development. Low mtDNA copy number due to a Tfam knockdown lead to an energy deficit during embryogenesis and an imbalance between mtDNA replication, transcription and 
translation, which can explain the (mitochondrial) stress response and pathology observed. The mitochondrial stress pathway described can have an important role in diseases associated with mitochondrial dysfunction.

In chapter 7, a general discussion is provided on the findings in this thesis. The mitochondria with their own genome were indispensable for the success of eukaryotic development and the existence of complex, multicellular organisms, such as human beings. This success had a price, which comprises mitochondrial and mtDNA-related disorders, infertility and (accelerated) ageing-related diseases. The studies described in this thesis contribute significantly to identification and understanding of the underlying mechanisms that define mitochondrial inheritance through the bottleneck, as well as its role in the occurrence of de novo mtDNA mutations. This is of key importance to identify the risk of women having children with mtDNA disease, as well as to counsel and treat these patients. Furthermore, understanding of the evolutionary mechanisms helps to increase the safety of innovative (reproductive) treatments, such as nuclear genome transfer as well as options to treat mtDNA-related female infertility.

The zebrafish provides an excellent model to increase our knowledge on mtDNA genetics, as it combines invertebrate genetic engineering advantages with vertebrate physiology in a high number of samples. As described in this thesis, the zebrafish model is useful to unravel the underlying mechanisms in the inheritance of mtDNA mutations through the bottleneck. Furthermore, the model can be well applied to study the impact of both nDNA and mtDNA mutations in the development of mitochondrial disorders. Lastly, the zebrafish is well-suited to identify and test possible therapeutic compounds to cure and ameliorate mitochondrial disorders. As "nothing in biology makes sense, except in the light of evolution", studies, as described in this thesis, contribute to the development of better counselling and therapeutic options of mitochondrial disorders. 


\section{Samenvatting}

Mitochondriële ziekten omvatten een groep van erfelijke aandoeningen of syndromen, met vaak een dodelijke afloop. Patiënten kunnen een breed scala aan klinische symptomen ontwikkelen. Meestal ontstaan mitochondriële ziekten door een defect in de oxidatieve fosforylering (OXPHOS), de voornaamste bron van cellulaire energie (in de vorm van ATP). In totaal ontwikkelen ongeveer 1 op 5.000 mensen een mitochondriële ziekte. Organen met een hoge energiebehoefte zijn het meest vatbaar voor een defect in de OXPHOS, waardoor mitochondriële aandoeningen zich voornamelijk manifesteren in de hersenen en de skeletspieren. Naast deze klinische heterogeniteit, is ook het genetische spectrum van mitochondriële aandoeningen erg heterogeen, omdat mutaties in verschillende genen kunnen lijden tot vergelijkbare fenotypes. Bovendien liggen degenen die coderen voor eiwitten meteen mitochondriële functie verspreid over twee verschillende genomen. Een deel van de genen liggen op het nucleaire DNA, terwijl de rest het erfelijk materiaal van de mitochondriën zelf grotendeels omvat: het mitochondrieel DNA (mtDNA). Het mtDNA verschilt in allerlei opzichten van zijn nucleaire tegenhanger. Het mitochondriële genoom komt voor in meerdere kopieën en cellen handhaven een hoog mtDNA kopie-aantal. Hierdoor kan een mix onstaan van mtDNA dat wildtype is of dat varianten of mutaties bevat, een verschijnsel dat bekendstaat als heteroplasmie. Een ziekte veroorzaakt voor een mtDNA mutatie manifesteert zich alleen wanneer het heteroplasmie-niveau (of mutatiepercentage) boven een bepaalde drempelwaarde uitkomt. Ook de manier van overerving verschilt van de nucleaire tegenhanger. Waar de overerving van het nucleaire DNA plaatsvindt volgens de wetten van Mendel, erft het mtDNA exclusief over van moeder op kind. Tijdens deze overerving treedt een 'bottleneckeffect' op: de lichaams- en geslachtscellen van de nakomelingen ontvangen slechts een beperkt aantal mtDNA moleculen van de moederlijke eicel. Hoe de bottleneck precies werkt, is op dit moment nog onbekend.

De algehele doelstelling van deze thesis is om de processen te karakteriseren die ten grondslag liggen aan het bottleneckeffect, alsmede om het ontstaan van nieuwe mtDNA ziektes te verklaren. Specifieke doelen zijn:

- om de grootte van het mtDNA bottleneckeffect tijdens de overerving te bepalen;

- om het risico in te schatten waarmee nieuwe mtDNA tijdens de eicelontwikkeling ontstaan;

- om de biologische processen te identificeren die ten grondslag liggen aan de (mutatie-specifieke) veranderingen in de mtDNA mutatiepercentages tussen moeders en kinderen;

- om het effect te bestuderen van een laag aantal mtDNA kopieën op de embryogenese.

Hoofdstuk 2 geeft een overzicht van de evolutie van mitochondriën en het mitochondriële genoom. De verspreiding van mitochondriële genen over twee verschillende genomen is het resultaat van de manier waarop eukaryoten zijn ontstaan. De huidige hypothese is dat eukaryoten zijn ontstaan door een endosymbiose van twee prokaryoten, beide met een eigen genoom. De ene prokaryoot, een waterstofafhankelijke anaerobe archaebacterie, fungeerde hierbij als gastheer voor een ademende eubacterie, waardoor de gastheer grote hoeveelheden intracellulair 
waterstof-afhankelijke adenosine trifosfaat (ATP) kon gaan produceren. De eubacterie werd uiteindelijk een mitochondrium met zijn eigen genoom, het mtDNA. Het nadeel was een toename in de productie van intracellulaire reactieve zuurstofverbindingen (ROS - reactive oxygen species) met een hoge mutagene activiteit, waardoor een hoge mutatiefrequentie in het mtDNA ontstaat. Volgens de ratchet theorie van Müller, zorgt een opeenhoping van mutaties in een aseksueel overgedragen genoom, zoals het mtDNA, voor een achteruitgang in de reproductiviteit en uiteindelijk uitsterving. Dit is met het mitochondriële genoom niet gebeurd, omdat er verschillende mechanismen bestaan die de accumulatie van mutaties in het mtDNA voorkomen. Vroeg in de evolutie is een groot gefdeelte van de genetische informatie vanuit het mtDNA overgebracht naar de kern (dit proces staat bekend als EGT - endosymbiotic gene transfer). Na de fylogenetische afsplitsing van dieren, schimmels en planten, zijn binnen deze rijken verschillende, aanvullende adaptaties geselecteerd die het schadelijke effect van de hoge mutatiefrequentie verminderden, waardoor het mtDNA van dieren, schimmels en planten andere kenmerken hebben. Het mtDNA genoom van dieren is compact, telt een vast aantal genen, komt voor in een groot aantal kopieën en ondergaat nauwelijks recombinatie, terwijl het mtDNA van planten veel groter is, met een variabel genenaantal, een laag kopie-aantal en veel recombineert. Het mtDNA van schimmels lijkt op beide. Planten houden de mutatiefrequentie laag door middel van een effectieve bescherming tegen ROS enerzijds en een goed herstel van mtDNA fouten door recombinatie anderzijds. Deze mechanismen zijn veel minder ontwikkeld in dieren, die de hoge mutatiebelasting opvangen door middel van een hoog aantal mtDNA kopieën in combinatie met het wegfilteren van mutaties door de bottleneck tijdens de overerving. Dieren hebben evolutionair gezien een hogere mutatiefrequentie van het mtDNA, wat als voordeel heeft dat zij zich beter kunnen aanpassen aan veranderingen in hun omgeving ten aanzien van mitochondriële energieproductie en daardoor kunnen migreren. Voor planten geldt dit niet. Bij dieren is het hoge aantal mtDNA kopieën en de bottleneck van cruciaal belang om de balans te bewaken tussen positieve, negatieve en neutrale mtDNA varianten. Het bottleneckeffect is sterk geconserveerd in het rijk der dieren, wat het belang van het proces voor het behoud van een gezond mtDNA genoom onderstreept.

De bottleneck kenmerkt zich door een beperking in het aantal mtDNA moleculen dat overerft vanuit de eicel naar de nakomelingen, waardoor een sampling-effect ontstaat. Dit filtert mtDNA mutaties met een laag mutatiepercentage uit. De grootte, timing, variatie en mogelijke verschillen tussen kiembaan -en nietkiembaancellen zijn niet bekend. Om hier meer inzicht in te krijgen hebben we in hoofdstuk 3 de zebravis als model gebruikt om de mtDNA bottleneck gedetailleerd in kaart te brengen. Door de zebravis te gebruiken kunnen we grote aantallen (onrijpe) eicellen, kiemcellen (PGCs - primordial germ cells) en niet-kiemcellen verzamelen tijdens verschillende fases van de embryonale ontwikkeling. Rijpe zebravis-eicellen bevatten gemiddeld $\sim 19.0 \times 10^{6}$ kopieën van het mtDNA, met grote variatie tussen eicellen. Tijdens de vroegste stadia van de embryogenese, verdelen de mtDNA kopieën zich over de dochtercellen en repliceert het mtDNA niet. Hierdoor neemt het aantal kopieën af tot 170 mtDNA moleculen in een PGC, overeenkomen met wat in muizen is gevonden, en tot $\sim 50 \mathrm{mtDNA}$ moleculen in de niet-kiemcellen. Op een vast moment in de ontwikkeling bevatten de cellen het laagste aantal mtDNA kopieën, waardoor de variatie in de eicel bepalend is voor de hoeveel mtDNA moleculen die er dat punt in de cellen aanwezig zijn. 
Om uit te zoeken of het lage mtDNA kopie-aantal, dat ontstaat door de bottleneck, ervoor zorgt dat sommige eicellen meer gevoelig zijn voor het ontstaan van nieuwe (de novo) mutaties die een functioneel relevant heteroplasmieniveau bereiken, hebben we door middel van next-generation sequencing (NGS) de mtDNA sequentie bepaald in 103 eicellen afkomstig van acht verschillende zebravissen (hoofdstuk 4). Door gebruik te maken van statistische en biologische filters kunnen we varianten met een heteroplasmie-niveau $\geq 1.5 \%$ betrouwbaar detecteren. Ongeveer $\sim 20 \%$ van de eicellen hadden een de novo mutatie, die willekeurig verspreid over het mtDNA lagen. Dit kwam overeen met onze bevindingen in hoofdstuk 3, waar bleek dat $\sim 20 \%$ van de kiembaancellen als laagste aantal 73 of minder mtDNA kopieën bezitten. Mutaties die op dit punt ontstaan leiden tot detecteerbare mutatieniveaus. De frequentie waarmee de novo mutaties ontstaan, komt overeen met de foutfrequentie van het mitochondriële replicatie-enzym polymerase gamma (POLG). Dit maakt het aannemelijk dat replicatiefouten de voornaamste bron van mutaties is. Bovendien geeft het ook een mogelijke verklaring voor de hoge mutatiefrequentie ( 25\%) waarmee de novo mtDNA mutaties in de mens worden gevonden.

Op basis van de verdeling van het mutatiepercentage van drie mtDNA mutaties (m.3243A>G, m.8993T>G en m.14487T>C) in restmateriaal (oocyten, zygoten en embryo's) verkregen van verschillende humane draagsters die preimplantatie genetische diagnostiek hebben ondergaan, hebben we in hoofdstuk 5 de processen die ten grondslag liggen aan de bottleneck verder ontrafeld. Als we ervan uitgaan dat random genetische drift het effect van de bottleneck bepaalt, dan hebben de verschillende draagsters een bottleneck-grootte die varieert van 49-159 (voor de m.3243A $>$ G mutaties) tot 21 (voor de m.14487T >C mutatie) en 10 (voor de m.8993T>G mutatie). Deze waardes komen overeen met onze waarnemingen in de zebravis in hoofdstuk 3. Drie observaties suggereren echter dat ook niettoevalsprocessen of selectie een rol spelen tijdens de overerving van het mtDNA: een correlatie tussen de hoogte van de m.3243A>G mutatie en de grootte van de bottleneck, de afwezigheid van mutatieniveaus boven de $80 \%$ bij de m.3243A>G mutatie en de consequent scheve mutatieverdeling bij de overerving van de m.8993T > G mutatie. Tijdens de eicelvorming en de vroegste embryonale ontwikkeling zijn cellen voornamelijk afhankelijk van OXPHOS voor hun energievoorziening en een verstoorde OXPHOS (bijvoorbeeld wanneer het mutatieniveau van de m.3243A>G mutatie boven de $80 \%$ uitkomt) maakt het onmogelijk voor cellen om te overleven. De restactiviteit van de OXPHOS in cellen die de m.8993T>G of m.14487T>C mutatie dragen blijkt echter voldoende om, zelfs bij een mutatieniveau van $100 \%$, genoeg energie te produceren, waarschijnlijk door het hoge mtDNA kopie-aantal. Tevens vindt er tijdens deze fase positieve selectie plaats voor mitochondriën met een hoog mitochondrieel membraanpotentiaal (MMP). Deze is het resultaat van het pompen van protonen over het binnenste mitochondriële membraan. De MMP is verlaagd in de aanwezigheid van de meeste mutaties die het OXPHOS-proces negatief beïnvloeden. De m.8993T>G mutatie zorgt er echter voor dat de MMP juist hoger is in vergelijking met wild-type, mutatie-vrije, mitochondriën, waardoor een positieve selectie ontstaat voor mitochondriën met deze mutatie. De m.14487T >C mutatie heeft geen duidelijk effect op zowel de OXPHOS als de MMP, waardoor alleen genetische drift de verdeling van deze mutatie bepaalt. Het samenspel van genetische drift en mutatie-specifieke selectieprocessen bepalen dus de overerving van het mtDNA. Het is van belang dit mee te nemen bij kiezen van de juiste preventiestrategie. Bij PGD 
en nucleaire genoom transfer (NGT) bijvoorbeeld, is inzicht in deze processen voor de mutaties van belang om te kunnen bepalen bij welk mutatieniveau embryo's veilig kunnen worden teruggeplaatst.

Zoals beschreven in hoofdstuk 3, vindt er sterke mtDNA replicatie plaats tijdens de ontwikkeling van de eicel, waardoor een hoog mtDNA kopie-aantal ontstaat. Dit is van groot belang voor de reproductie, gelet op de correlatie tussen het aantal mtDNA kopieën en de fertiliteit en de levensvatbaarheid van eicellen en vroege embryo's. In hoofdstuk 6 hebben we het effect van een verlaging van het mtDNA kopie-aantal tijdens de vroege ontwikkeling bestudeerd door het verlagen (een zogenaamde knock down) van de mitochondriële transcriptiefactor A (Tfam), één van de eiwitten die de hoogte van het mtDNA kopie-aantal bepaalt, met behulp van morpholino oligonucleotiden (MOs). Zebravis embryo's, waar Tfam was gereduceerd, vertoonden een defect in de embryonale ontwikkeling, voornamelijk zichtbaar in het hart en de hersenen. Dit effect was het zichtbaar 4 dagen na de bevruchting (4 dpf days post fertilization). Deze embryo's hadden $50-80 \%$ minder kopieën van het mtDNA in vergelijking met controle-embryo's. Met behulp van microarrays onderzochten we het effect van de Tfam knockdown op de expressie van alle bekende genen op $4 \mathrm{dpf}$. Zebravissen met een Tfam knockdown hebben een verlaging in de expressie van genen die op het mtDNA liggen en coderen voor eiwitten van de OXPHOS. Van de genen die voor nucleaire eiwitten coderen, hadden zowel metabole genen, als genen belangrijk voor proliferatie tijdens embryogenese een verlaagd expressieniveau, terwijl genen met een functie in de celcyclus, in de cellulaire signaalprocessen die belangrijk zijn voor de embryonale ontwikkeling en de mitochondriële translatie juist een significante verhoogd expressieniveau hadden. In overeenstemming met de expressieverhoging van genen uit de Fas pathway (stress inductoren van heat shock proteins), vonden we ook een verhoging van specifieke genen betrokken bij de mitochondriële unfolded protein response, enkele mitochondriële matrix protease genen en verschillende kerngenen betrokken bij apoptose. Hieruit concluderen we dat behoud of toename van het mtDNA kopie-aantal cruciaal is voor een normale embryonale ontwikkeling. Een verlaagd mtDNA kopie-aantal door een Tfam knockdown leidt tot een energietekort tijdens embryogenese en een disbalans tussen mtDNA replicatie, transcriptie en translatie, die de (mitochondriële) stressreactie en geobserveerde pathologie verklaren. De beschreven mitochondriële stress respons kan een belangrijke rol hebben in ziekten die gepaard gaan met een dysfunctie van de mitochondriën.

Hoofdstuk 7 omvat een algemene discussie over de resultaten van dit proefschrift. De mitochondriën met hun eigen genoom waren onmisbaar voor eukaryoten om zich succesvol te ontwikkelen tot complexe, meercellige organismen, zoals de mens. Dit succes had een prijs, die bestaat uit mitochondriële en aan het mtDNA-gerelateerde ziekten, infertiliteit en ouderdomsziekten. De studies zoals beschreven in dit proefschrift leveren een belangrijke bijdrage aan het begrijpen van de mechanismen die de overerving van mitochondriën via de bottleneck bepalen, evenals hun rol in het ontstaan van de novo mtDNA mutaties. Dit is van groot belang voor het inschatten van het risico dat vrouwen een kind met een mtDNA ziekte krijgen en het counselen en behandelen van deze patiënten. Verder zorgt een beter begrip van de evolutionaire mechanismen voor een verbetering van verschillende innovatieve therapieën, zoals NGT.

De zebravis is als model uiterst geschikt om onze kennis van het mtDNA 
te vergroten, omdat het eenvoudige genetische manipulatie mogelijk maakt in een gewerveld dier. Zoals te lezen in deze thesis, is de zebravis nuttig gebleken om meer inzicht te krijgen in het bottleneckproces en kan het bovendien worden gebruikt om zowel nucleaire als mtDNA mutaties te bestuderen. Tenslotte is de zebravis ook nog geschikt om nieuwe therapeutische stoffen die mitochondriële ziektes kunnen verlichten of genezen, te identificeren en te testen. Ook de zebravisstudies beschreven in dit proefschrift dragen daarom bij aan de ontwikkeling van betere opties tot counseling en therapie van mitochondriële aandoeningen. 



\section{Valorisation (I)}

\section{Zebrafish Flow Arena}

Monitor

Auke Otten, Jo Vanoevelen, Tom Theunissen, Bianca van den Bosch, Bart Beulen, Frank Nijpels, Marc Muller, Emile Arnoldussen, Hubert Smeets

Published as final report of the alma-in-silico project (Interreg IV program) 


\section{Summary}

Over the past decades the zebrafish (Danio rerio) has developed as an important organism in developmental genetics, neurophysiology and biomedicine ${ }^{1}$. Currently, the zebrafish model is also used to study complex behaviour, such as addiction, anxiety, or physiological pattern and age-related diseases ${ }^{2}$. As with mitochondrial disorders, for most of these diseases, a change in movement (such as heartbeat or eye movement) or swimming is a fundamental phenotype. To study these movement phenotypes in zebrafish larvae up to 7 days post fertilization (dpf), equipment exists, like the Zebrabox and Daniovision, which allow automatic monitoring and recording of heartbeat, blood flow, motility and activity in 96-well plates ${ }^{3}$. The zebrafish larvae activity can be modulated by light/dark period and by drugs. Although already wellestablished there is a need to extend the read-out parameters by including muscle loading/physical challenges to study exercise intolerance, a fundamental phenotype in ageing and various genetic disorders, including the mitochondrial disorders, and different stress triggers to study behavioural phenotypes. Alongside the studies presented in this thesis, we have aimed, together with Maastricht Instruments BV, to develop these modules for this growing market, which can be incorporated into existing Zebrabox hardware and commercialized together with the manufacturer of these systems. However, the modules should be optimized and validated.

\section{Introduction}

Maastricht Instruments BV (MI) is a spin-off company of Maastricht University Medical Centre, which is specialized in the development of innovative tools and instruments for research purposes in the field of Life Sciences. As such, MI has developed platform technology to measure accurately behaviour, including physiological aspects of test subjects, patients and animal models under various conditions. This platform technology can be modified or extended to accommodate the needs within specific research projects. As there is a strong tendency in science and society to reduce investigations in rodents and larger animal models, smaller and ethically more acceptable models, like zebrafish, are becoming more and more popular. Zebrafish are generally studies as larvae, for which no ethical approval is required, but also as adult fish. Ml recognized a potential market in these developments to extend its portfolio of measuring behaviour to new animal models, like zebrafish.

The applicability of the zebrafish in the field of genetic diagnosis derives from their perfect reverse genetic tools. Important to this development is the Zebrafish Mutation Project (ZMP) from the Wellcome Trust Sanger Institute, which aims to create a knockout allele in every protein coding gene of the zebrafish genome ${ }^{4}$ and will be made available to the scientific community. This is a major advance for genetic screenings. The studies presented in this thesis have indicated that mitochondrial genetics and function are well conserved in the zebrafish, which underlines their utility in studying mitochondrial disorders. In addition, the zebrafish model is also used to study complex behaviour, such as addiction, anxiety, or physiological pattern and age-related diseases ${ }^{2}$. Although zebrafish movement is central is almost all these conditions, current zebrafish monitor systems are limited in tracking zebrafish 
movement in standing water and offer a limited number of stimulations modalities (application of light is common, whereas options for electrical stimulation are limited) ${ }^{5}$. A zebrafish monitor is required, which has the possibility to perform muscle-loading tests and has a more extensive implementation of various stimulation modalities. It is our goal to develop a module, compatible with the Zebrabox, that enables exercise (in)tolerance test, to further complement the zebrafish as a disease model. In this part of the valorisation we describe the development of a zebrafish flow arena monitor, with a focus on the commercial and societal impact.

\section{Methods}

Because of the existing experience with the ZebraLab software in combination with the ViewPoint Zebrabox system it has been decided not to start the development of a complete zebrafish monitor system but to develop a new module, which can be incorporated in existing systems. The existing system has sufficient space at the sampling position to accommodate a customized microplate. Minor adjustments might have to be made to the housing to accommodate supply and drain tubes. The flow entering the compartments will be generated by means of an accurately controllable pump. The microplate will be designed such that similar flow conditions occur in all the compartments. Computational Fluid Dynamics (CFD) computations and measurements in prototype microplates will be applied to validate the flow field. The system will be designed such that zebrafish can easily be transferred to the compartments and that the microplates can easily be switched. The flow system will be designed to reduce possibilities for fluid soiling to a minimum. Zebrafish should be kept in fluid with a constant temperature of approximately $28^{\circ} \mathrm{C}$. Water quality is guaranteed by the use of filters and a UV lamp. The flow system will be designed to control both the temperature of the supply of water towards the microplate and the microplate itself accurately. The Zebrabox hardware will be applied to record the motion of the zebrafish and larvae, subsequently. The motion (speed/pattern) will be recorded by means of the ZebraLab software. Control of the stimulation modalities are integrated into the ZebraLab software.

\section{Results}

An evaluation of the potential market for these devices was performed and resulted in the removal of adult zebrafish from our designs since a flow-tube for adult fish already exists on the market. Hence, we focused on solutions for zebrafish larvae.

\section{Proof-of-principle flow system}

For the exercise (in)tolerance test, we have developed a flow tube proof-of-principle. It consists of a device which can be inserted in the Zebrabox and allows accurate control of temperature and water flow rate. The device consists of a series of parallel flow tubes (Figure 1) and water treatment unit (Figure 2). Herein, the zebrafish embryos can undergo a protocol for forced swimming against an increasing flow rate. 
In this way, critical swimming speed (standard measure of swimming performance) can be determined in real-time. Multiple flow tubes are placed in the field of view of the monitoring system. The flow entering the compartment is generated by means of an accurately controllable pump (Figure 2). At the inlet of the tubes the water enters at a specific flow rate. A plug-like flow is established by directing the water through a set of static mixers to disperse the flow over the surface of the tube (Figure 1), thereby generating a turbulent flow. This turbulent flow is led through a special filter that creates a laminar plug-like velocity. Zebrafish are only capable of swimming in this area in order to monitor their behaviour. The tube is part of a system that provides flow and temperature control (Figure 1).

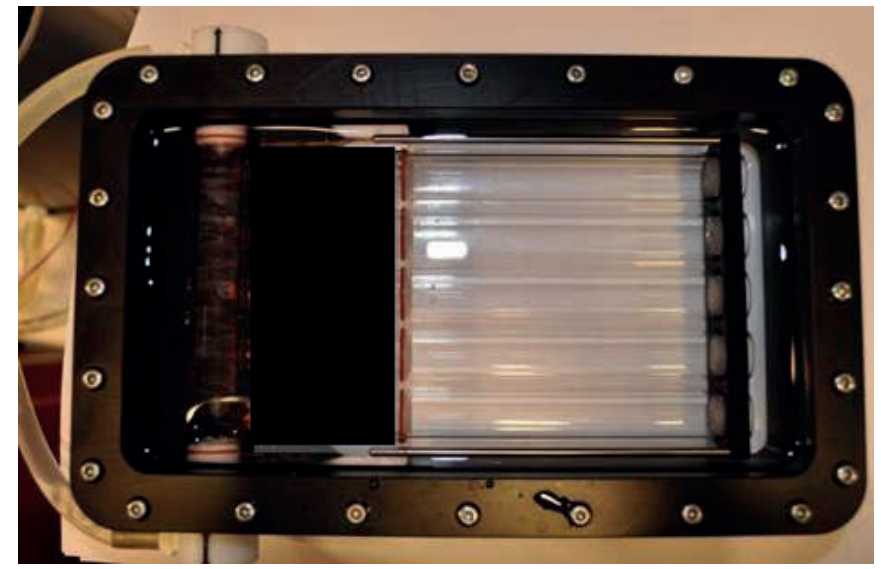

Figure 1 | Proof-of-principle exercise intolerance testing device. Water flow enters at the left site, and static mixers (blocked for IP reasons) and a special filter create a laminar-plug flow in the transparent area. In this area, zebrafish are capable of swimming as this is placed in the focus of the Zebrabox monitoring system.

\section{Optimization and validation}

The performance of the first prototype flow chambers has been evaluated and adapted accordingly. The ease of use and the applicability for use with zebrafish embryos was tested. Furthermore, the system with the flow chambers has to be made compatible with the use of the on-board camera. For this the flow chambers will be housed in a compartment filled with water to avoid issues with the differences in refractory index of water, glass and air. 


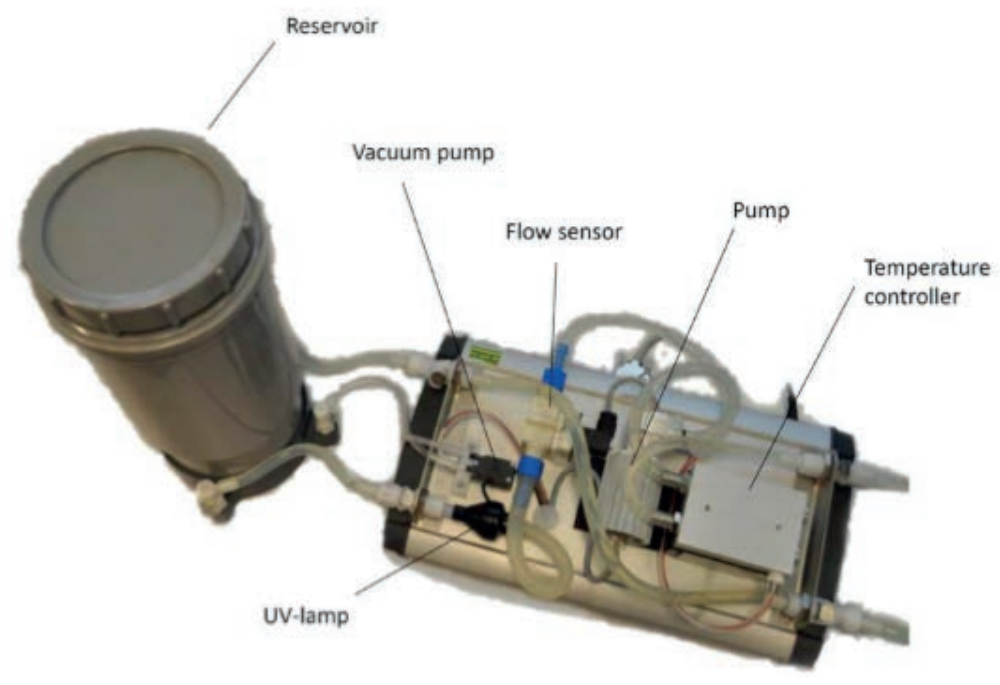

Figure 2 | Proof-of-principle design water treatment unit. Water reservoir and flow/temperature controller are assembled in two units. The water reservoir unit contains a tank which feeds the flow arena. The controller unit controls flow rate, temperature and water quality (UV sterilization).

We have validated the system by treating embryos with compounds that inhibit cellular energy production (inhibitors of OXPHOS). Clear differences in swimming behaviour performance in treated and control groups could be demonstrated (Figure 3 ). The proof-of-principle consists of a device that is capable of determining exercise intolerance in zebrafish embryos.

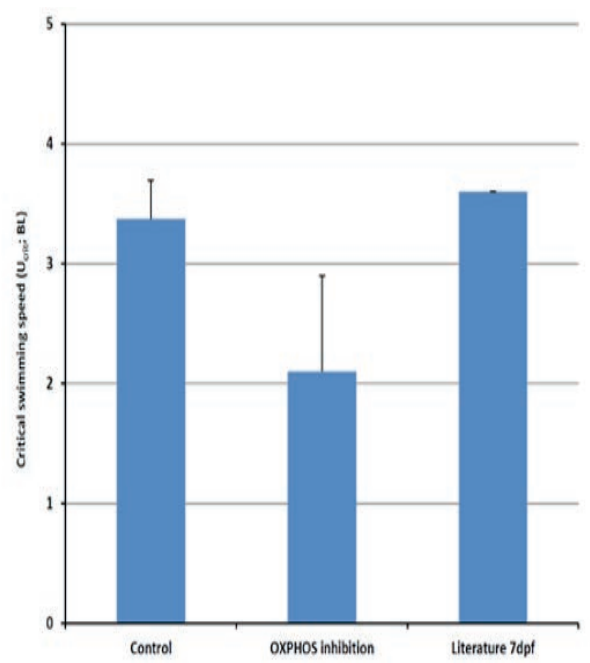

Figure 3 | Validation of the exercise intolerance test. Embryos (5 dpf) were incubated in water with or without an OXPHOS inhibitor $\left(\mathrm{NaN}_{3}\right)$, after which they were placed in the tubes to perform the exercise protocol. $U_{\text {crit }}$ values were lower in the embryo's that received OXPHOS inhibition. 


\section{Conclusions and commercial perspectives}

The exercise (in)tolerance device can be used as read-out for any genetic variant or compound affecting muscle power, mobility or exercise intolerance, like for example mitochondrial disorders. Over 800 laboratories worldwide carry out research on zebrafish. After the validation phase the adapted prototypes are ready to be transformed into devices that can be mass produced and commercialized. To this end, a feasibility study to evaluate the possibility for patent application is performed by MI. Ml expects with this new product to extend its portfolio of platform technology to measure accurately behaviour including physiological aspects of test subjects, patients and animal models under various conditions, to an exciting new model organism with considerable market potential.

\section{References}

1. Lessman, C.A. (2011). The developing zebrafish (Danio rerio): a vertebrate model for highthroughput screening of chemical libraries. Birth Defects Res C Embryo Today 93, 268-280.

2. Norton, W., and Bally-Cuif, L. (2010). Adult zebrafish as a model organism for behavioural genetics. BMC Neurosci 11, 90.

3. Prober, D.A., Rihel, J., Onah, A.A., Sung, R.J., and Schier, A.F. (2006). Hypocretin/orexin overexpression induces an insomnia-like phenotype in zebrafish. The Journal of neuroscience : the official journal of the Society for Neuroscience 26, 13400-13410.

4. Kettleborough, R.N., Busch-Nentwich, E.M., Harvey, S.A., Dooley, C.M., de Bruijn, E., van Eeden, F., Sealy, I., White, R.J., Herd, C., Nijman, I.J., et al. (2013). A systematic genome-wide analysis of zebrafish protein-coding gene function. Nature 496, 494-497.

5. Winter, M.J., Redfern, W.S., Hayfield, A.J., Owen, S.F., Valentin, J.P., and Hutchinson, T.H. (2008). Validation of a larval zebrafish locomotor assay for assessing the seizure liability of early-stage development drugs. J Pharmacol Toxicol Methods 57, 176-187. 



\section{Valorisation (II)}

Scientists and

Science Journalism

Auke BC Otten 
Science as subject of societal discussions has gained a lot of popularity over the last years. In many areas of science, interesting developments, e.g. in the field of biotechnology, neuroscience and cancer treatments, have created an increasing attention by the media. In the Netherlands, the television show "De wereld draait door (DWDD)" invites scientists on a regular basis. In the spin-off "DWDD University", special attention to a scientific topic is given by means of a live college by a renowned scientist. Scientists like Robert Dijkgraaf and Erik Scherder have given enthusiastic and inspiring colleges on scientific subject with for a broad audience. While science always has been discussed in small communities, such developments has made science accessible for a broad public. As a result, science has become more popular. Science is complicated and highly specialized, with many experts in their own focusses field of research. In order to keep science accessible for both colleagues in different field as the broad public, I believe that scientists should be trained in the basics of journalism in order to be able to share their knowledge to both colleagues and the broader public.

As scientist, I decided to follow a course on Science Journalism from SCW in Amsterdam. During 12 course days, scattered over 4 months, I was trained in writing for a broad public (including newspapers, magazines, online blogs etc.) about science in order to contribute to responsible awareness of the public on scientific matters. During this course, I have written many different scientific stories, which were criticised by trained science journalists. I have learned to acquire a clearer and direct writing style when writing about science. The course has contributed to my development as scientist and made me aware of the societal role that scientists have, as we are mostly paid by public money. When scientist act in public, such as a television show, an enthusiastic, clear and direct communication about science will contribute to the trust society places in science, as it enables a more transparent view on the scientific process.

Besides this, the course was also useful for my scientific writing. The tendency in most journals to write about science in their specific jargon, mostly in complicated and long sentences. Direct writing, which benefits the public, also benefits our colleagues. It will allow a better understanding of the science that has been carried out, which allows a better check on the underlying scientific process. Also, in line with the expectation to prepare a short description for the laymen alongside with the project application, aspects from journalism in scientific writing are becoming more and more indispensable. Lastly, as I have experienced that scientific journals are increasingly expecting author summaries, graphical abstracts and even movies to further illustrate the scientific paper, the journalistic tools I learned have been of great value for my scientific career. 

Credits 
In een promotietraject leer je een hoop. Vanzelfsprekend op het gebied van de wetenschap, maar veel meer nog over jezelf. Het is absoluut waar dat je jezelf op gezette momenten tegenkomt en je verbaast over hoe je op bepaalde situaties reageert. Ik ben helemaal niet het type dat graag alleen op pad gaat, en juist mijn promotietraject vroeg om een intensieve samenwerking met de université de Liège, een eindje verderop, bij onze Zuiderburen. In veel opzichten is dat toch ver weg, met een cultuur -én taalbarrière. Ondanks alle moeite die het me heeft gekost, ben ik nu blij en trots op de goede samenwerking die er met deze universiteit is ontstaan. En heb ik ook daar veel meer geleerd dan alleen "werken met zebravisjes". Zo zijn er nog veel leermomenten op te noemen, van het doen van experimenten tot het geven van presentaties, maar ook over het organiseren van dagjes-uit, borrels en andere sociale experimenten. Uiteindelijk is organiseren wel het toverwoord in je promotie.

Het heeft heel wat voeten in de aarde gehad om een proefschrift voor elkaar te krijgen en omdat je je dagelijks begeeft tussen allerlei gepromoveerden is het soms moeilijk om te bevatten dat het afleveren van een proefschrift iets is om even bij stil te staan. Dan besef je dat er een hele berg mensen zijn die daar, ieder op geheel unieke wijze, een bijdrage aan hebben geleverd. Want promoveren doe je niet alleen, dat mag duidelijk zijn. Er zijn mensen die je de kansen geven; de vrijheid en het vertrouwen om je te ontplooien, om je weg te zoeken en te vinden. Er zijn mensen die je adviezen willen geven, die met je mee willen denken. Mensen die je gewoon even gelijk durven te geven (of juist niet) als je ergens over twijfelt. Mensen die je een hand toesteken op het lab of bij een analyse; die je net even wat werk uit handen nemen. Of die taxichauffeur speelden om in Münster of Mainz vissen op te gaan halen. En dan nog de mensen die je er gewoon even "doorheen slepen", die je laten lachen op momenten dat het einde verder weg leek dan ooit, en je een duwtje in de rug geven.

Op deze plaats in het proefschrift wil ik al die mensen bedanken. Bedanken voor het feit dat jullie, elk op jullie unieke manier, hebben bijgedragen aan dit eindresultaat, een eindresultaat waar ik absoluut trots op ben. Ik kies ervoor om jullie te bedanken door het noemen van jullie naam, omdat eenieder van jullie wel weet hoe dankbaar ik ben voor de manier waarop een bijdrage is geleverd aan dit proefschrift. Een proefschrift is soms net als een film, met goede afloop, dus ik kies voor het format van de aftiteling van een film om jullie te bedanken. We zien elkaar vast wel weer ergens, binnen of buiten de wetenschap, en ik wens jullie allemaal oprecht het allerbeste.

Liefs,

Auke 


\section{The impact of the mitochondrial bottleneck on mtDNA disease risk and embryogenesis}

Regie

Bert Smeets

Regieassistentie

Marc Muller

Beoordelingscommissie

Joep Geraedts

Erik Biessen

Wout Lamers

Patrick Chinnery

Franck Dequiedt

Paranimfen

Iris Boesten

Ellen Lambrichs

(Ex-)Roomies

Mike Gerards

Ivo Eijkenboom

Tom Theunissen

Florence van Tienen

Romy Lutgens

An Voets

Rita Brandão

Minh Nguyen

Ruben Martherus

ClinGen-Crew

Erika Timmer

Bieke Vanherle

Jo Vanoevelen

Barbie Machiels

Rick Kamps

Richard Dohmen

Janine Grashorn

Ruby Zelissen

Rowida El Momami

Jo Beerens

Rianne Timmer

Roy Janssen

Tim Kampermann

Bart de Koning

Ondersteuning

Rosy Engelen

Els Claessen

\section{Zebravis-crew}

Marie Winandy

Josien Derhaag

Hélène Pendeville

Raafat Stephan

Erez Raz

Katsiaryna Tarbashevich

Sandra Ormenese 


\section{Statistiek \& bio-informatica}

Fons Stassen

David Samuels

De "overkant"

Bianca van den Bosch John Engelen Jos Dreesen

\section{Met dank aan}

René de Coo

Jan-Willem Taanman

Bart Beulen

Petra van der Molen Jos Broers

\section{Family \& Friends}

Bernard Otten

Frank van Opdorp

Maaike Koonen-Otten

Emiel van der Vorst

Lennert Coumans

Irene Eurlings

Thomas Hoeksema

Koen Souren

Demi Hellemons

Marten Hoeksema
Michiel Adriaens

Radek Szklarczyk

Suzanne Sallevelt

Alexandra Hendrickx

Marion Drüsedau

Evelien Smeets

Maud Starmans

Mieke Henfling

Henny Schürmann
Patrick Lindsey

Lars Eijssen

Debby Hellebrekers

Frank Beijers
Marie Herbert

Frank Nijpels

Maastro-Lab

Philippe Lambin
Jo en Willy van Opdorp Jacq en Lily Vrusch

Mirelle Huijskens

René Hoeksema

Etienne Jumpertz

Bas Koonen

Hetty en Herman

Bram Schmitz

Onno Otten

Oma

Mam
Anita Brouns

Travis en Parascha

Rob van Opdorp

Bert en Metha Hoeksema

Peter Lem

Sanne van Tongelen

Bonnie Tilmans

Seppe Koonen

Pap

In warme herinnering aan

Maurice van Opdorp 

Appendix

Curriculum vitae 
Auke Otten wordt op 3 juli 1986 geboren te Sittard. Van 1998 tot 2005 doorliep hij zowel het Hoger Algemeen Voorbereidend Onderwijs (HAVO) als het Voorbereidend Wetenschappelijk Onderwijs (VWO) aan het Romboutscollege te Brunssum. Door zijn liefde voor de humane biologie start hij in 2005 met de bacheloropleiding Moleculaire Levenswetenschappen aan de universiteit Maastricht. Na een afstudeerstage bij de afdeling Toxicologie studeert hij in 2008 cum laude af. Vervolgens start hij met de masteropleiding Cardiovascular Biology and Medicine, tevens aan de universiteit Maastricht. Zijn afstudeerstage vindt plaats bij de subsectie Clinical Genomics van de afdeling Genetica en Celbiologie. Hij onderzoekt de mogelijkheden van de zebravis als model voor het bestuderen van mitochondriële processen in ziekte en gezondheid. Van het Prinses Beatrixfonds ontvangt hij hiervoor een scholarship (€2000) voor een bezoek aan het laboratorium van Dr. Jan-Willem Taanman, University College London, voor het leren van technieken voor celkleuringen, en het uitvoeren van onderzoek in de zebravisfaciliteit van de Université de Liège. Hij behaalt in 2010 zijn mastertitel. Vervolgens wordt hij aangesteld bij dezelfde groep op een AIO-project, gefinancierd door GROW (School for Oncology and Developmental Biology), maar verricht hij zijn onderzoek deels op de Université de Liège in België. Tijdens zijn promotie onderzoekt hij de invloed van de mitochondriële bottleneck op het ontstaan van mitochondriële ziekten en op de embryogenese. Hij bezoekt meerdere toonaangevende congressen, zoals the 9th European conference on mitochondrial research (Euromit) in Tampere, Finland. Hiervoor ontving hij een reisbeurs van het Simonsfonds. Door zijn passie voor wetenschapscorrespondentie volgt hij in 2014 twaalf weken een cursus wetenschapsjournalistiek in Amsterdam. Sinds februari 2016 is hij werkzaam als postdoc bij de afdeling Maastricht Radiation Oncology (Maastro), waar hij zijn kennis van mitochondriën gebruikt om het ontstaan van toxiciteit na bestraling te kunnen voorspellen. 


\section{Wetenschappelijke publicaties}

Otten, AB, Smeets HJ, 'Evolutionary defined role of the mitochondrial DNA in fertility, disease and ageing.' Human reproduction update, 2015 Sep-Oct; 21(5):671-89

Otten, AB, Theunissen, TE, Lambrichs, EH, Winandy, M, Tarbashevich, K, Raz, E, Vanoevelen, JM, van den Bosch, BJ, Muller, M, Smeets, HJ, 'Differences in strength and timing of the mtDNA bottleneck between zebrafish germline and non-germline cells' Cell Reports 2016 Jul 19;16(3):622-30.

Otten, AB, Dohmen, RG, Adriaens, M, Kamps, R, Timmer, AJ, Vanherle, SJ, Boesten, IB, Muller, M, Gerards, M, Stassen, AP, Smeets, HJ, 'Zebrafish with a low mtDNA copy number are at risk of de novo mutations reaching functional significance' Genetics; Manuscript accepted

Otten, AB, Sallevelt, SC, Carling, PJ, Dreesen, JC, Drüsedau, M, Spierts, S, Paulussen, AD, Chinnery, PF, Samuels DC, Smeets, HJ, 'Genetic drift and selection on OXPHOS capacity and mitochondrial activity drive mtDNA bottleneck size and mutation distribution in embryos.' Manuscript submitted

Otten, AB, Lambrichs, EH, Vanherle, SJ, Voncken, A, Pendeville, H, Winandy, M, Vanoevelen JM, Lindsey, P, Gerards, M, Muller, M, Smeets, HJ, 'Effect of decreased mtDNA replication during embryonic development.' Manuscript in preparation.

\section{Presentaties op congressen}

American Society of Human Genetics (ASHG) Annual Meeting, Vancouver (CA), 2016 Otten, AB, Sallevelt, SE, Dreesen, JC, de Die, C, Paulussen, AD, Theunissen, TE, Winandy, M, Lambrichs, E, Eijkenboom, I, Gerards, M, Vanoevelen, JM, Tarbashevich, K, Raz, E, Samuels, D, van den Bosch, BJ, Muller, M, Smeets, HJ, 'The role of the mitochondrial bottleneck in mtDNA disease risk and embryogenesis in zebrafish (non-)germline cells and human preimplantation embryos.' Poster presentation

American Society of Human Genetics (ASHG) Annual Meeting, Baltimore (USA), 2015

Otten, AB, Sallevelt, SE, Dreesen, JC, de Die, C, Paulussen, AD, Theunissen, TE, Winandy, M, Lambrichs, E, Eijkenboom, I, Gerards, M, Vanoevelen, JM, Tarbashevich, K, Raz, E, Samuels, D, van den Bosch, BJ, Muller, M, Smeets, HJ, 'Characterization of the mtDNA bottleneck is human preimplantation embryos and zebrafish germline and non-germline cells.' Poster presentation selected for poster walk 
GROW Science day, Maastricht (NL) 2010, 2011, 2012, 2014 and 2015

Otten, AB, Theunissen, TE, Sallevelt, SE, Dreesen, JC, de Die, C, Paulussen, AD, Winandy, M, Lambrichs, EH, Gerards, M, Vanoevelen, JM, Samuels, D, van den Bosch, BJ, Muller, M, Smeets, HJ, 'The inheritance of mtDNA mutations - more than just gambling.' Selected oral poster presentation

$9^{\text {th }}$ European conference on mitochondrial research (Euromit), Tampere (FI), 2014 Otten, AB, Theunissen, TE, van den Bosch, BJ, Winandy, M, Muller, M, Vanoevelen, JM, Smeets, HJ, 'The mitochondrial DNA bottleneck in zebrafish and its role in evolution.' Poster presentation

\section{GIGA-Day, Liège (BE), 2014}

Otten, AB, Voncken, A, Stephan, R, Ormenese, S, Theunissen, TE, Winandy, M, Muller, M, van den Bosch, BJ, Vanoevelen, JM, Smeets, HJ, 'The mitochondrial DNA bottleneck in the somatic and primordial germ cells of the zebrafish.' Invited oral presentation

$8^{\text {th }}$ European Zebrafish Meeting, Barcelona (ESP), 2013

Otten, AB, Theunissen, TE, Winandy, M, Muller, M, van den Bosch, BJ, Vanoevelen, JM, Smeets, HJ, 'The mitochondrial DNA bottleneck in the somatic and primordial germ cells of the zebrafish.' Poster presentation

Genetics Retreat, Kerkrade (NL), 2013

Otten, AB, Theunissen, TE, Winandy, M, Muller, M, van den Bosch, BJ, Vanoevelen, JM, Smeets, HJ, 'The mitochondrial DNA bottleneck in the somatic and primordial germ cells of the zebrafish.' Oral presentation

XIV European congress of Ichthyology, Liège (BE), 2012

Otten, AB, Theunissen, van den Bosch, BJ, TE, Vanoevelen, JM, Winandy, M, Muller, $\mathrm{M}$, Smeets, HJ, 'Mitochondrial DNA inheritance in the zebrafish model.' Oral presentation

\section{Subsidies \& Prijzen}

Poster geselecteerd voor poster walk tijdens het jaarlijks congres van The American Society of Human Genetics (ASHG), 2015

Reisbeurs (€600,-) van het Simonsfonds (1052), 2014

Scholarship reisbeurs (€2000,-) van het Prinses Beatrixfonds voor spierziekten (SC10-05), 2010 
